\title{
Decision Diagrams for Discrete Optimization
}

\author{
John Hooker \\ Carnegie Mellon University \\ ICAPS Tutorial \\ London, June 2016
}




\title{
Companion Tutorial:
}

\section{Decision Diagrams for Sequencing and Scheduling}

\author{
Willem-Jan van Hoeve \\ Carnegie Mellon University
}

Immediately follows this tutorial 


\section{Decision Diagrams}

- Used in computer science and Al for decades

- Logic circuit design

- Product configuration

- A new perspective on optimization

- Constraint programming

- Discrete optimization 


\section{Decision Diagrams}

- Relevance to planning and scheduling:

- Naturally suited to dynamic programming formulations.

- State-dependent actions and costs.

- New method for defeating curse of dimensionality.

- Branch-and-bound solution. 


\section{Decision Diagrams}

- DDs have been used in planning literature...

- To encode (or approximate) state-dependent cost or cost-to-go.

- See yesterday's tutorial "Planning with State-Dependent Action Costs"

- By Robert Mattmüller and Florian Geißer.

- This tutorial presents DDs as a general optimization method. 


\section{Decision Diagrams}

- General advantages:

- No need for inequality formulations.

- No need for linear or convex relaxations.

- New approach to solving dynamic programming models.

- Very effective parallel computation.

- Ideal for postoptimality anaylsis

- Disadvantage:

- Developed only for discrete, deterministic optimization.

- ...so far. 


\section{Outline}

- Decision diagram basics

- Optimization with exact decision diagrams

- A general-purpose solver that scales up

- Relaxed decision diagrams

- Restricted decision diagrams

- Dynamic programming model

- A new branching algorithm

- Computational performance

- Modeling the objective function

- Inventory management example

- Nonserial decision diagrams

- References 


\section{Decision Diagram Basics}

- Binary decision diagrams encode Boolean functions

\begin{tabular}{ccc|c}
$x_{1}$ & $x_{2}$ & $x_{3}$ & $f$ \\
\hline 0 & 0 & 0 & 1 \\
0 & 0 & 1 & 0 \\
0 & 1 & 0 & 0 \\
0 & 1 & 1 & 1 \\
1 & 0 & 0 & 0 \\
1 & 0 & 1 & 0 \\
1 & 1 & 0 & 1 \\
1 & 1 & 1 & 1 \\
\hline
\end{tabular}

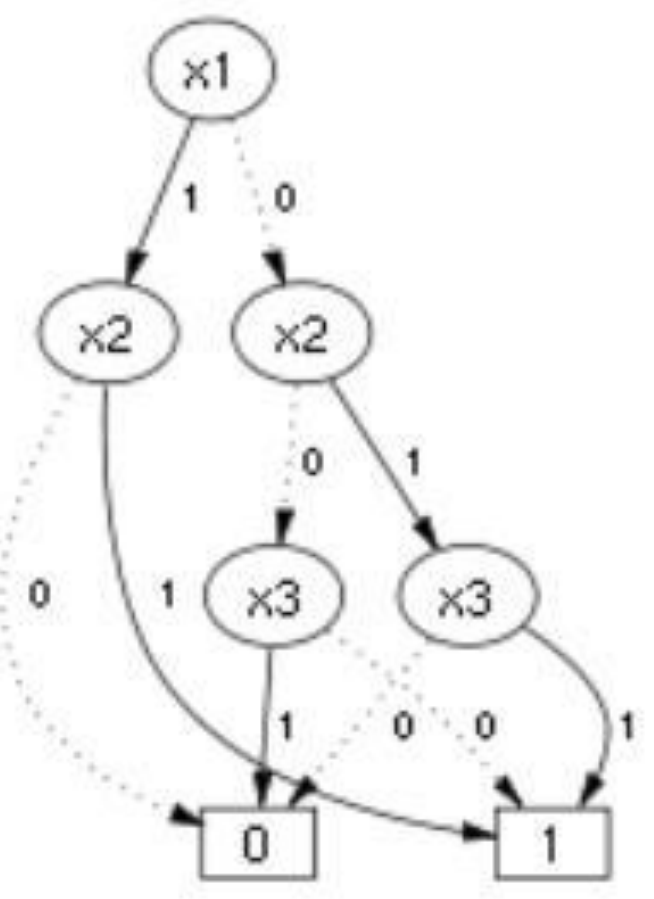




\section{Decision Diagram Basics}

- Binary decision diagrams encode Boolean functions

- Historically used for circuit design \& verification

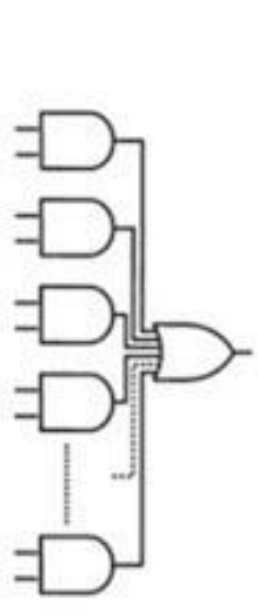

(a) Logic network

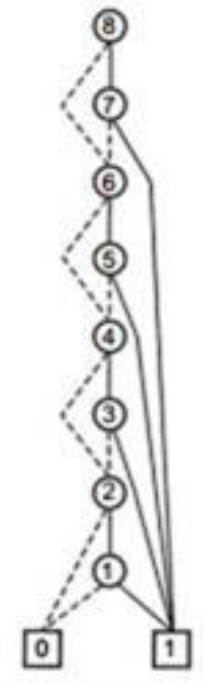

(b) BOD for varlables in the best order

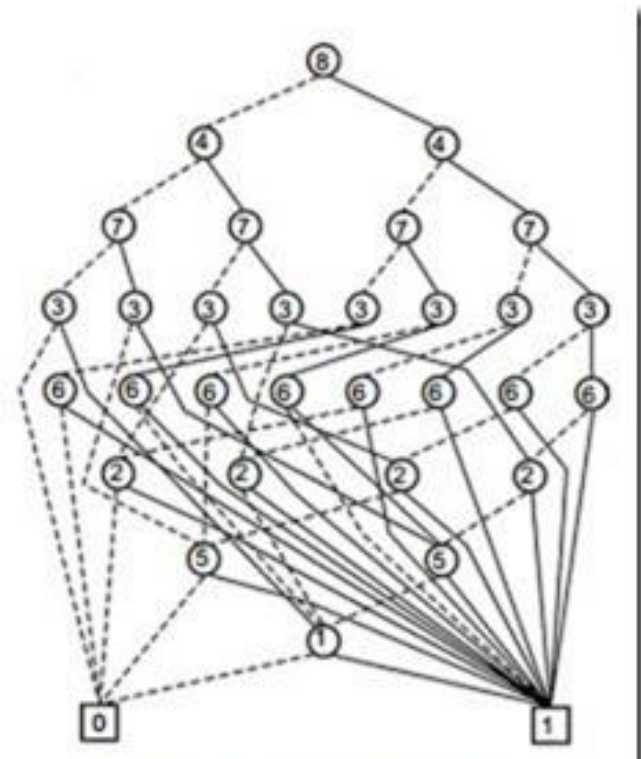

(c) BOD for variables in the worst order

FIGURE 29.11 BDDs for 2-levd logic network with AND/OR gates. 


\section{Decision Diagram Basics}

- Binary decision diagrams encode Boolean functions

- Historically used for circuit design \& verification

- Easily generalized to multivalued decision diagrams

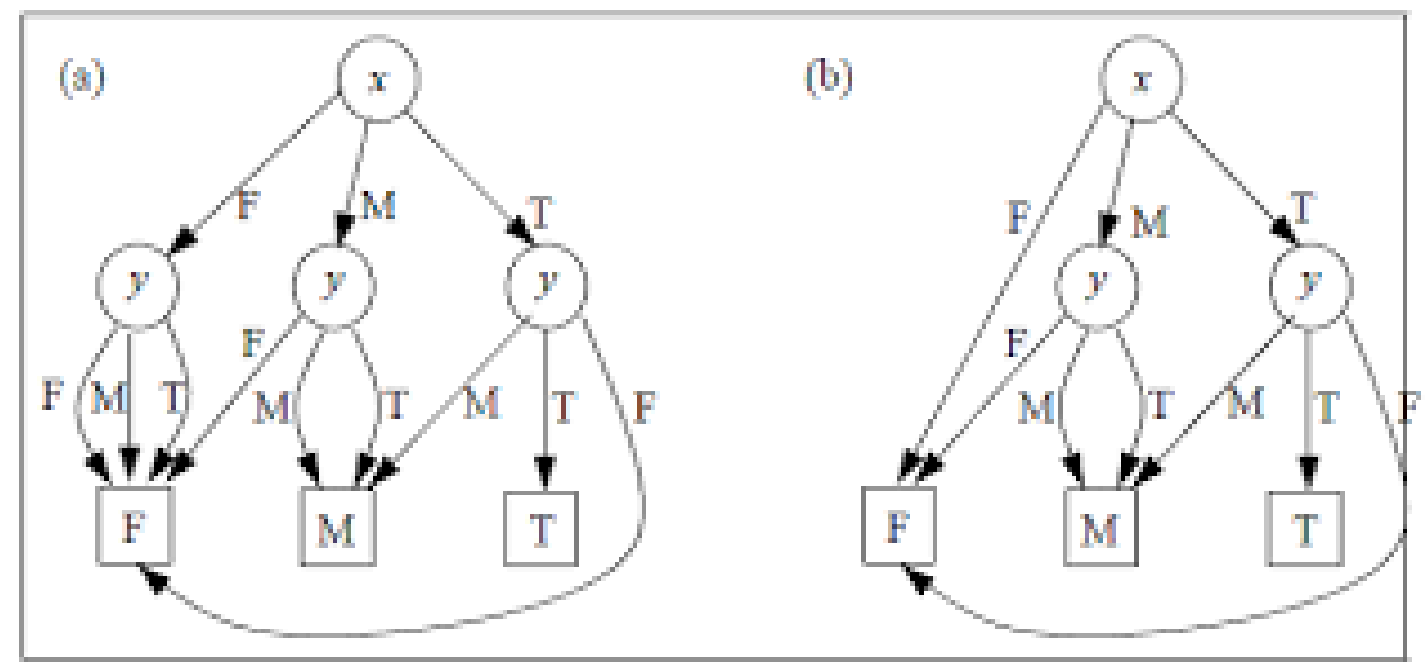




\section{Reduced Decision Diagrams}

- There is a unique reduced DD representing any given Boolean function.

- Once the variable ordering is specified.

\section{Bryant (1986)}

- The reduced DD can be viewed as a branching tree with redundancy removed.

- Superimpose isomorphic subtrees.

- Remove redundant nodes. 


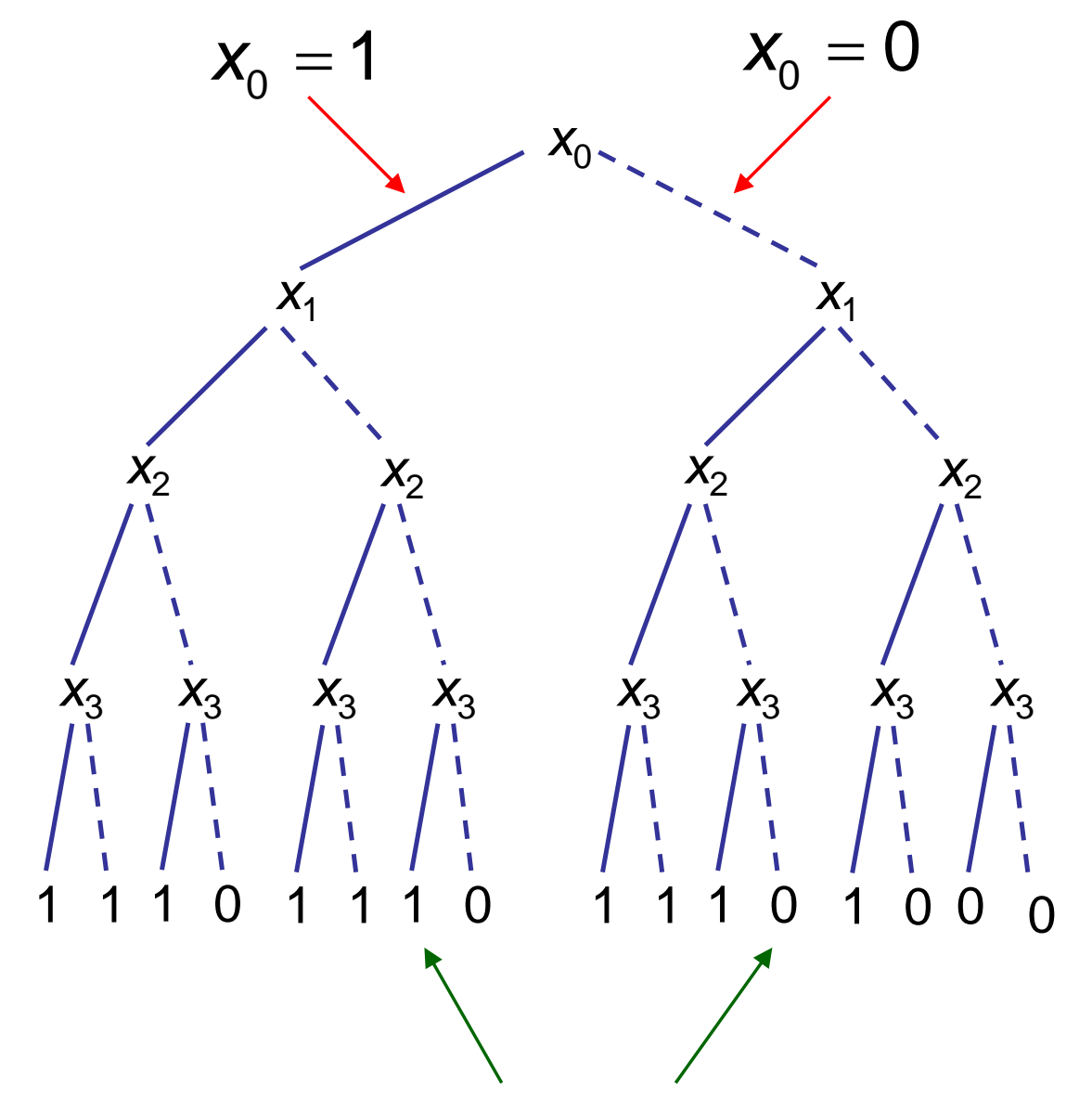

Branching tree for 0-1 inequality

$$
2 x_{0}+3 x_{1}+5 x_{2}+5 x_{3} \geq 7
$$

1 indicates feasible solution, 0 infeasible 


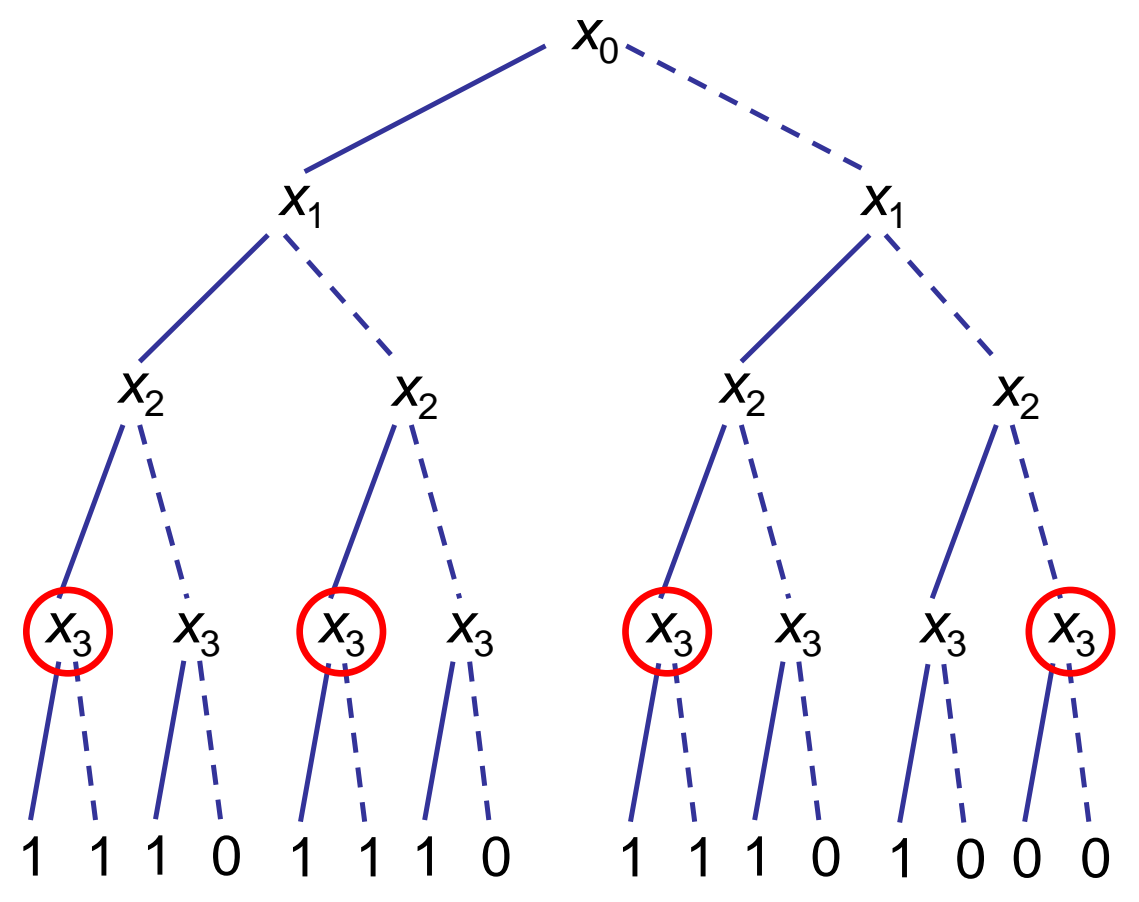

Branching tree for 0-1 inequality

$$
2 x_{0}+3 x_{1}+5 x_{2}+5 x_{3} \geq 7
$$

Remove redundant nodes... 


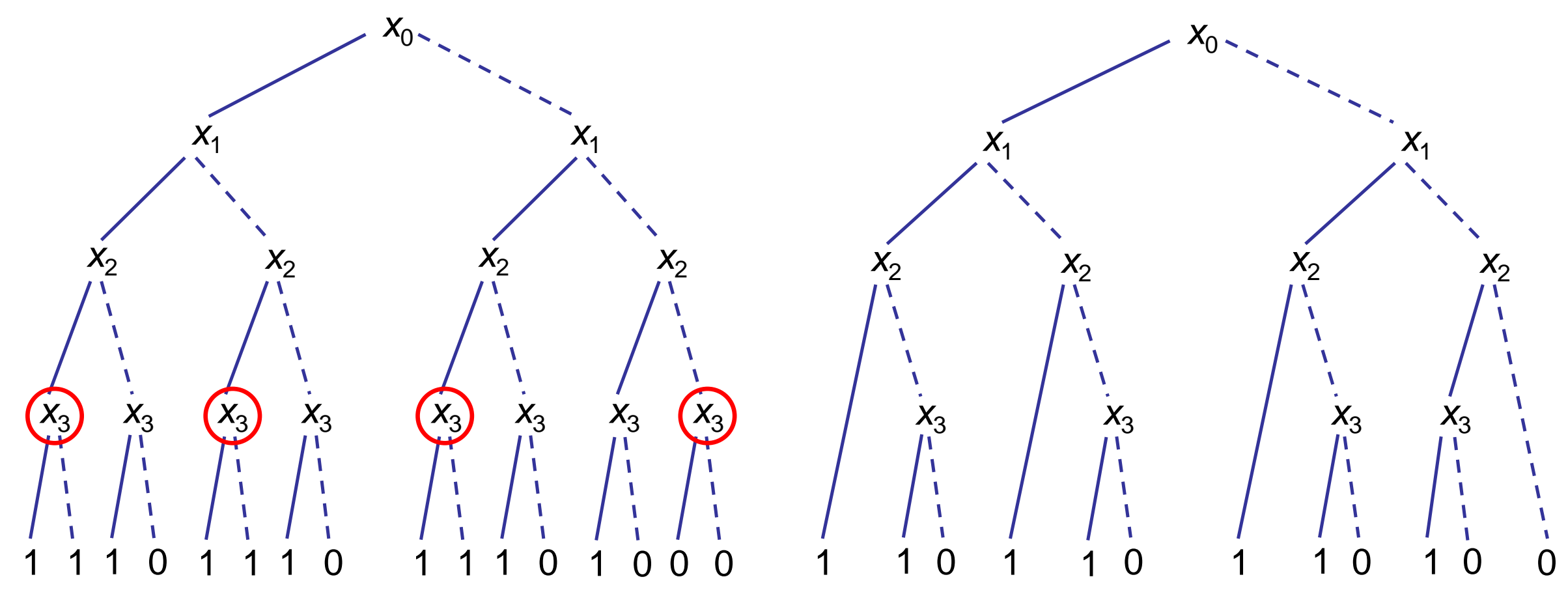


Superimpose identical subtrees...

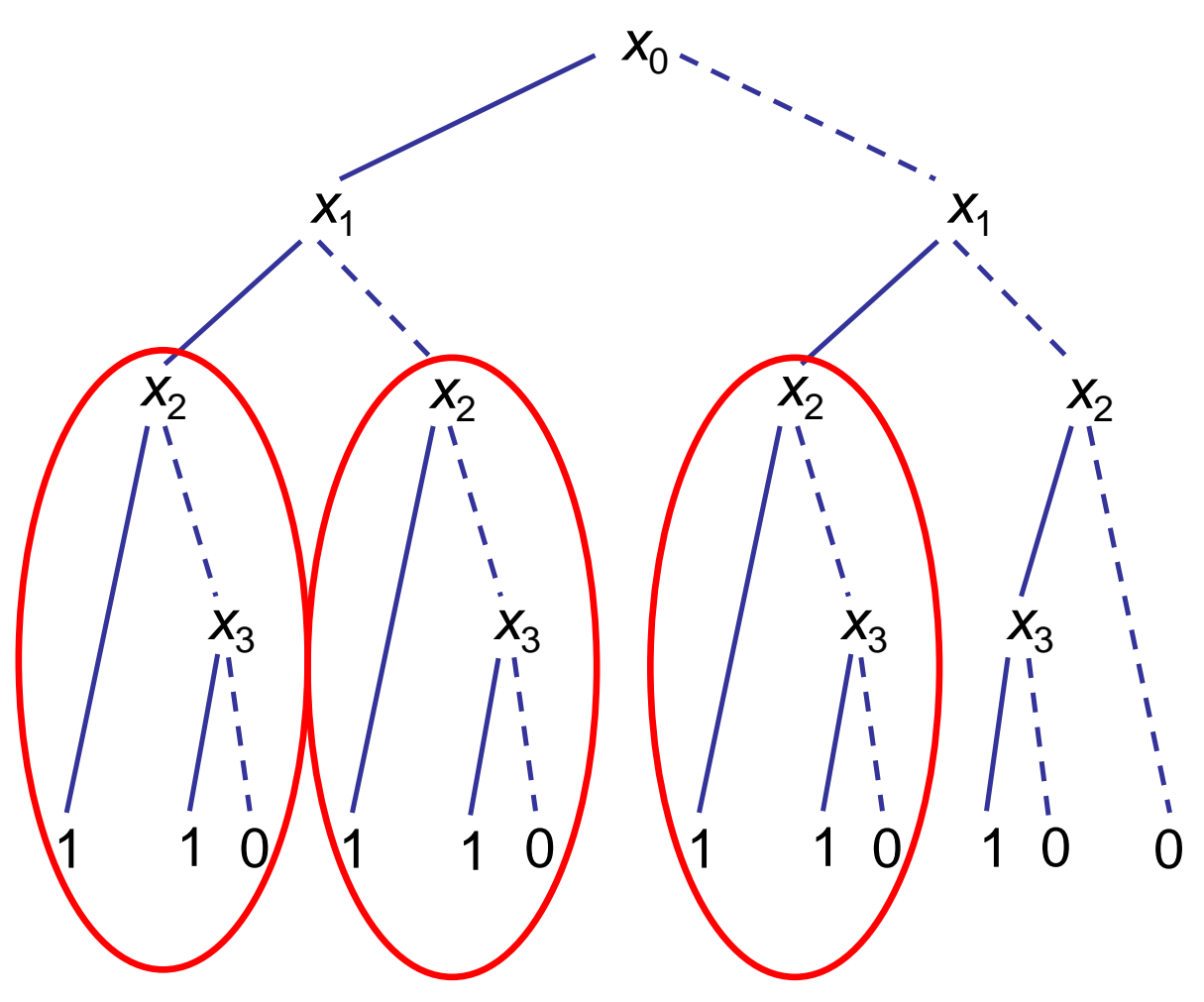



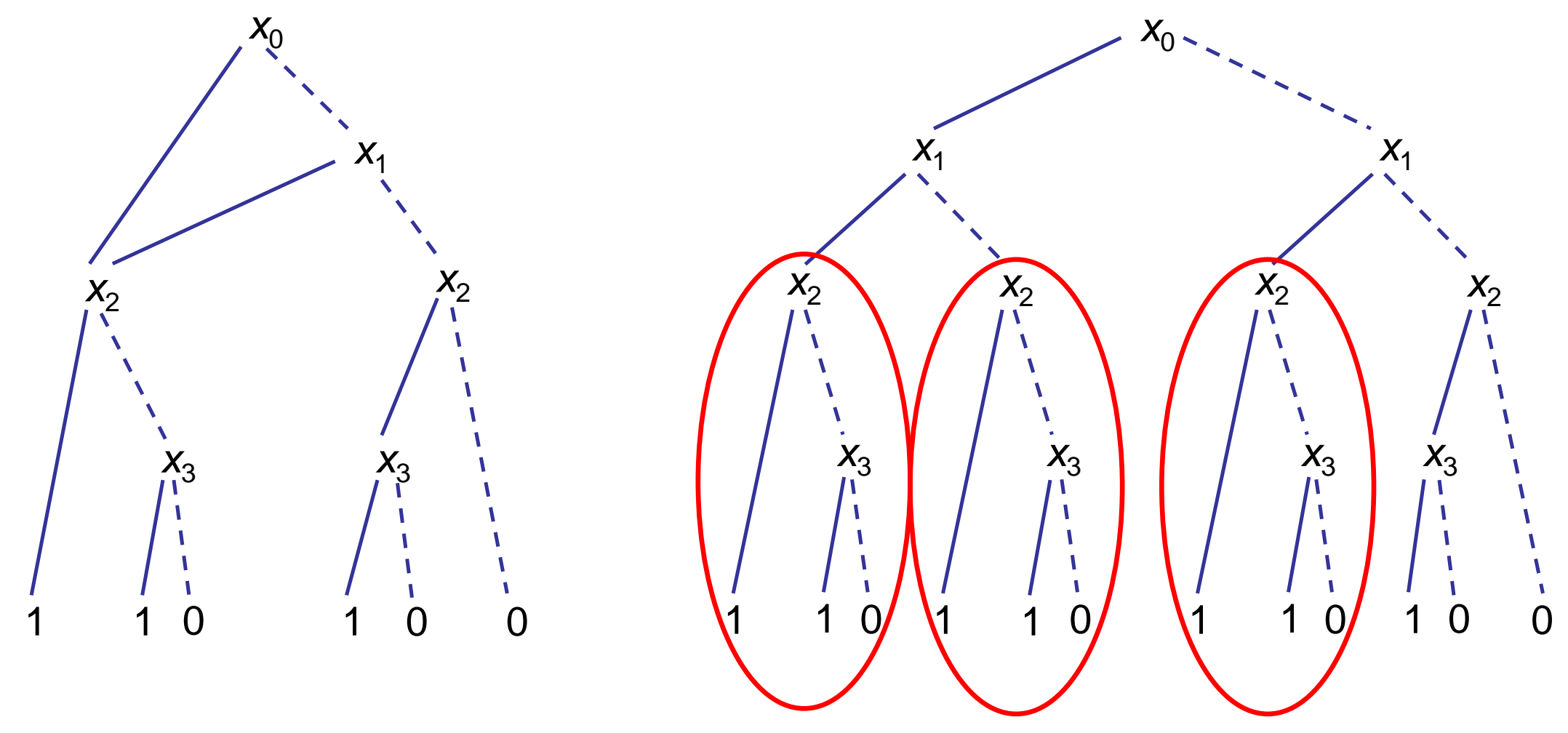


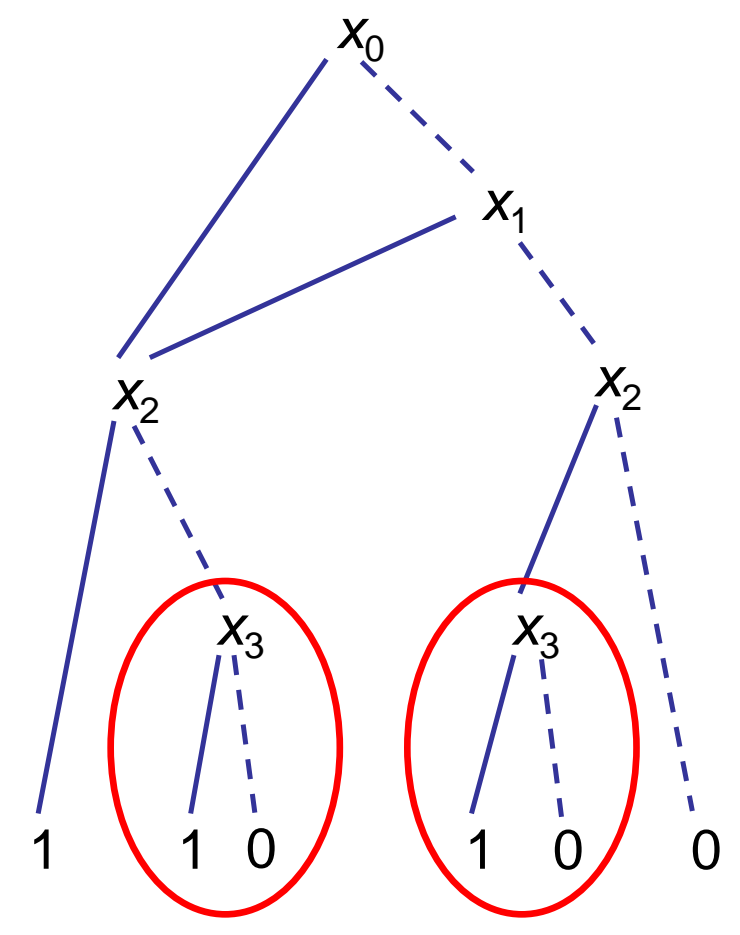

Superimpose identical subtrees... 

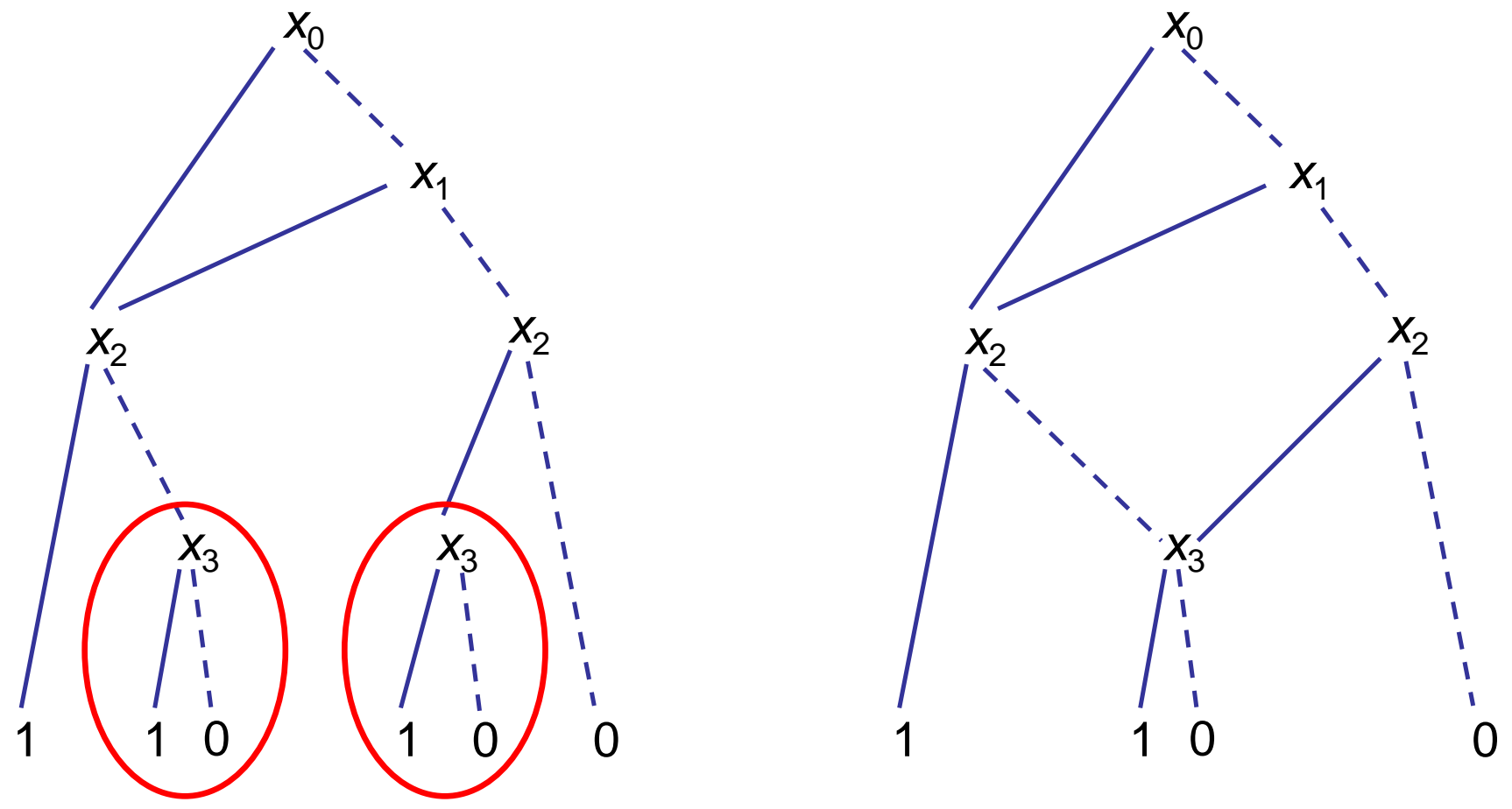
Superimpose identical leaf nodes...

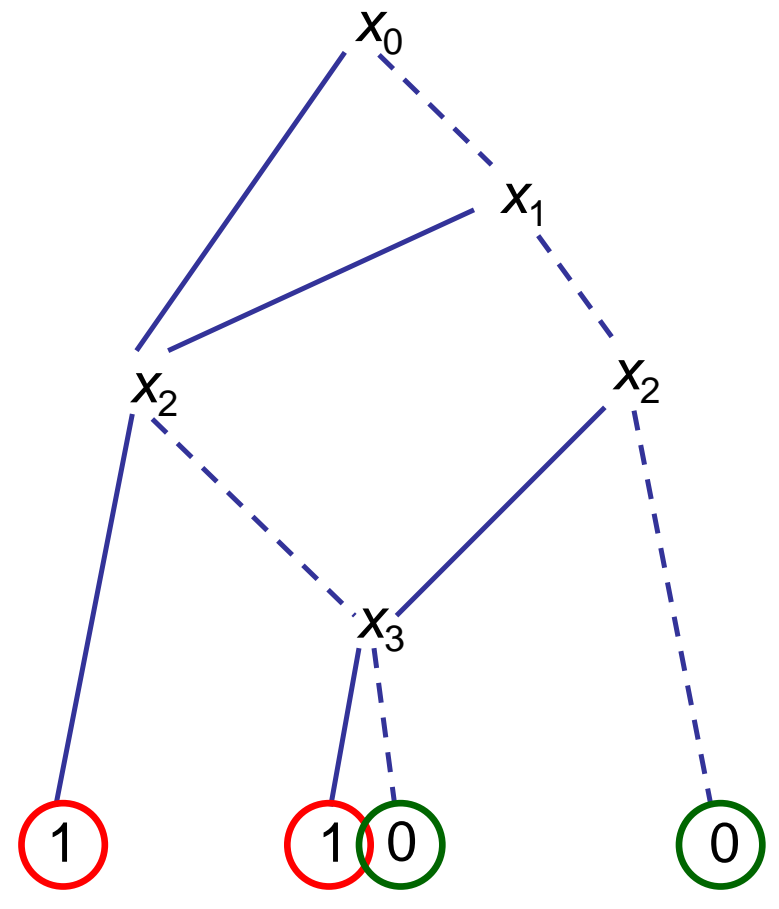




$$
\Leftrightarrow \Leftrightarrow
$$



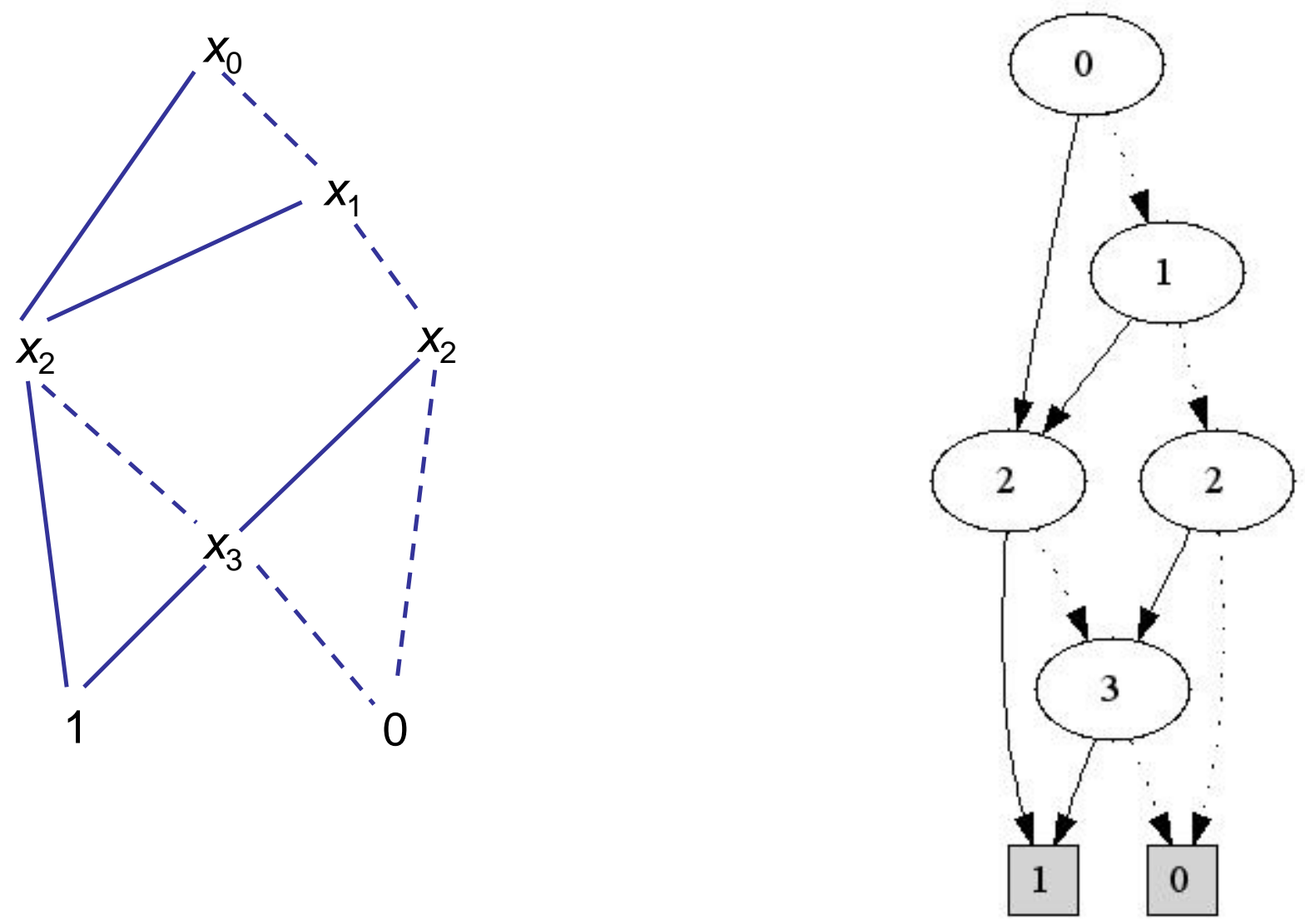

as generated by software 


\section{Reduced Decision Diagrams}

- Reduced DD for a knapsack constraint can be surprisingly small...

The $0-1$ inequality

$$
\begin{aligned}
& 300 x_{0}+300 x_{1}+285 x_{2}+285 x_{3}+265 x_{4}+265 x_{5}+230 x_{6}+230 x_{7}+190 x_{8}+200 x_{9}+ \\
& 400 x_{10}+200 x_{11}+400 x_{12}+200 x_{13}+400 x_{14}+200 x_{15}+400 x_{16}+200 x_{17}+400 x_{18} \leq 2700
\end{aligned}
$$

has 117,520 maximal feasible solutions (or minimal covers)

But its reduced BDD has only 152 nodes... 


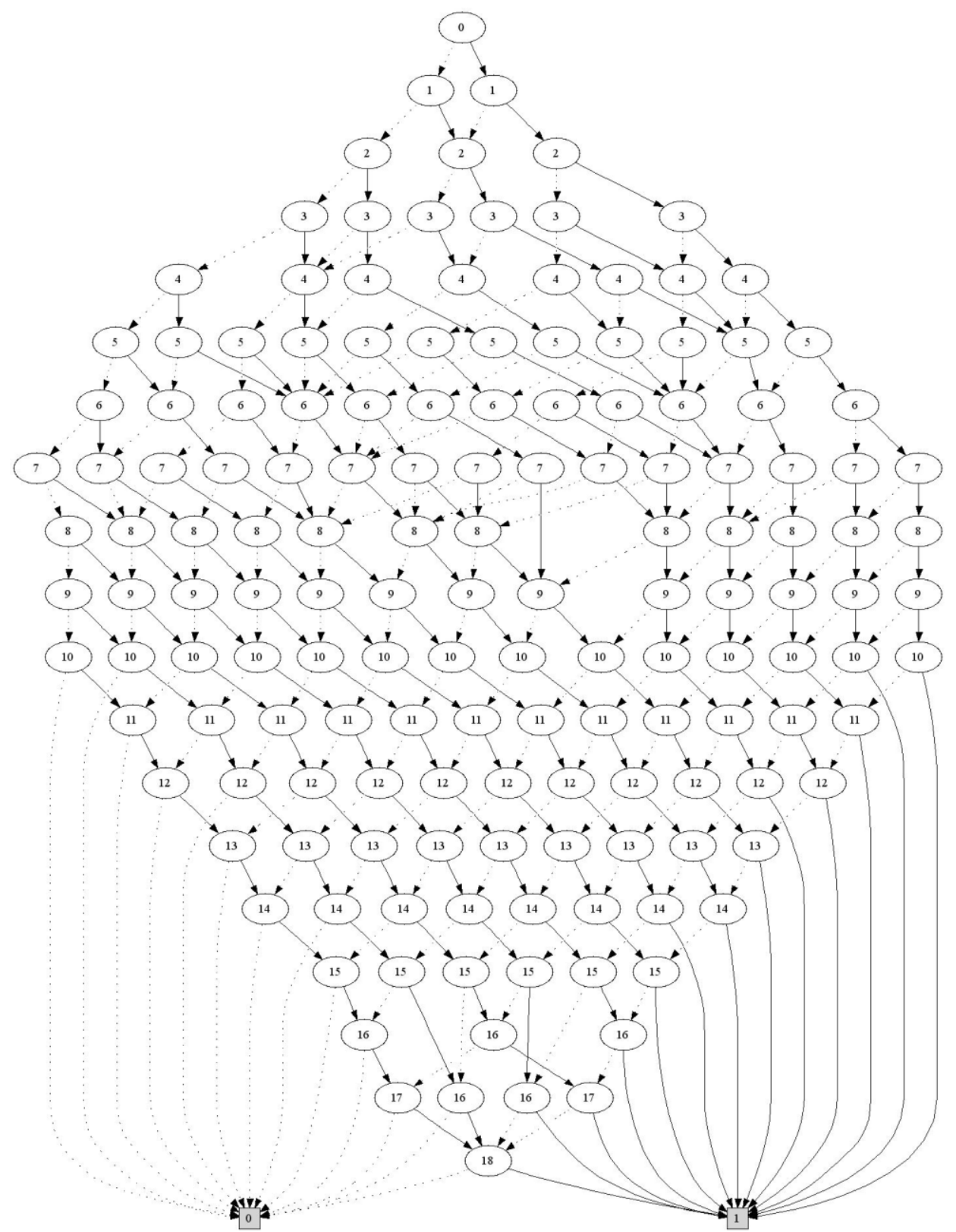




\section{Optimization with Exact Decision Diagrams}

- Decision diagrams can represent feasible set

- Remove paths to 0.

- Paths to 1 are feasible solutions.

- Associate costs with arcs.

- Find longest/shortest path

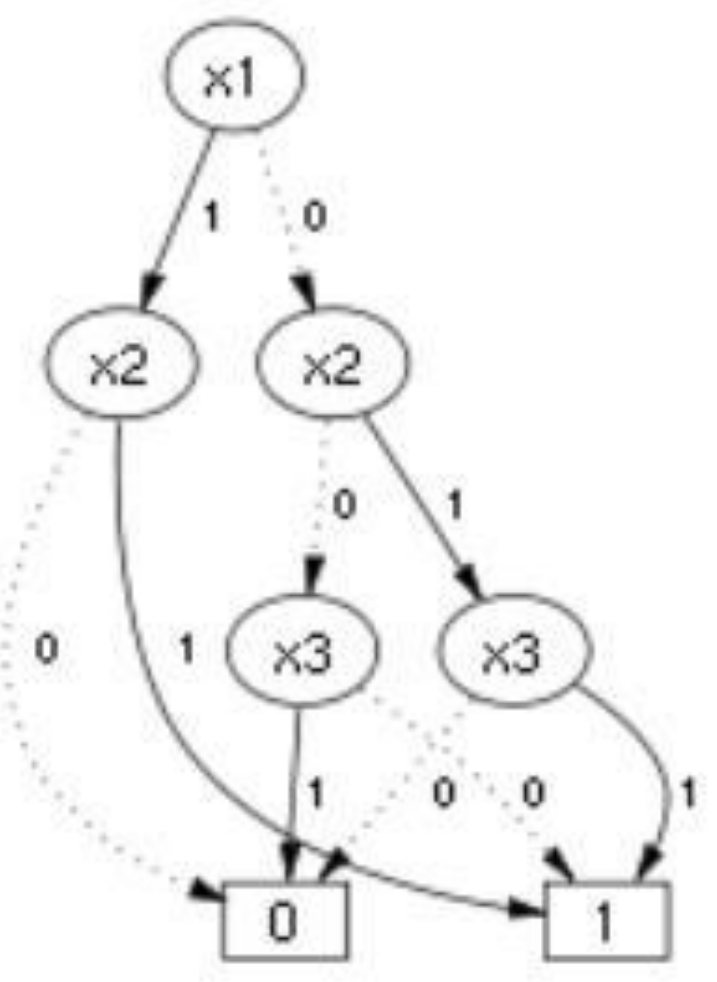




\section{Stable Set Problem}

Let each vertex have weight $w_{i}$

Select nonadjacent vertices to maximize $\sum_{i} w_{i} x_{i}$

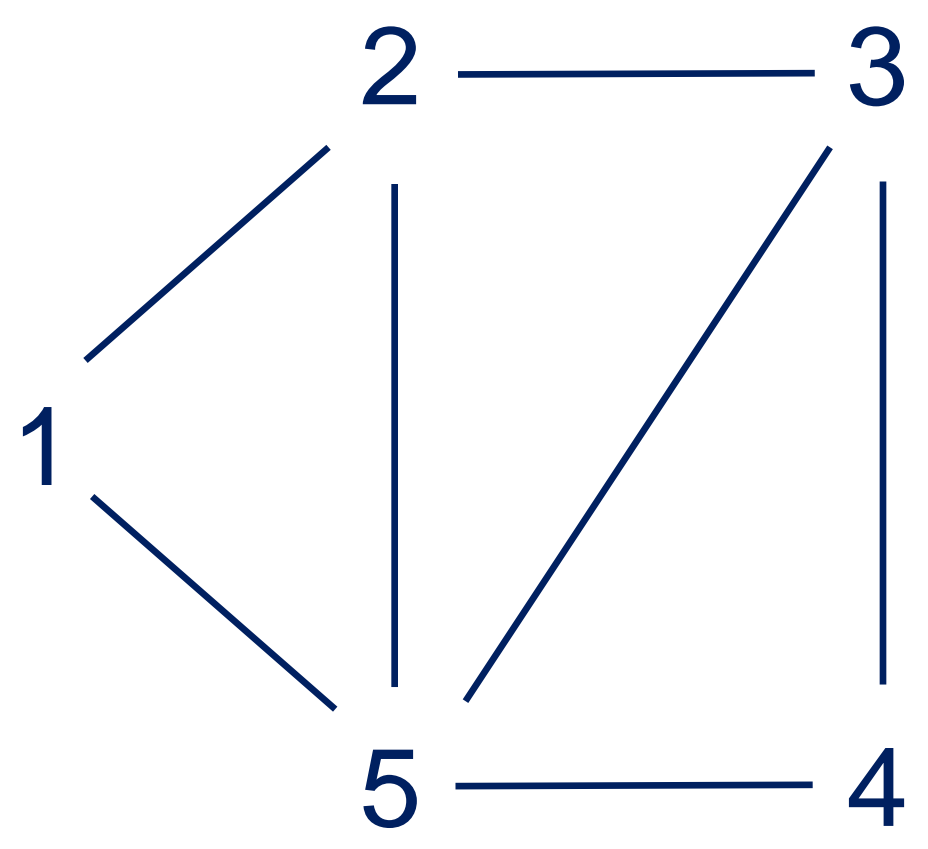



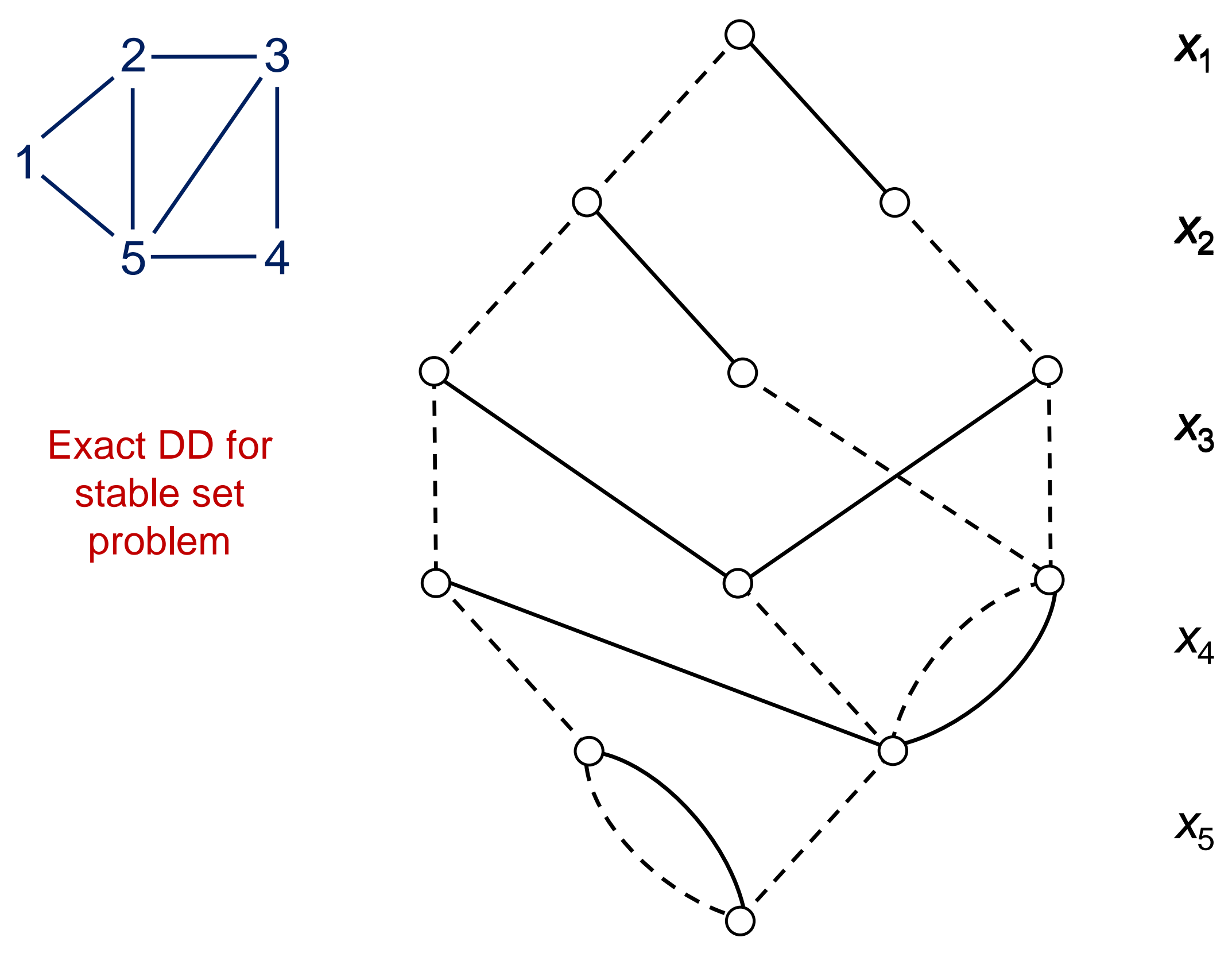

$x_{1}$

Exact DD for stable set problem 

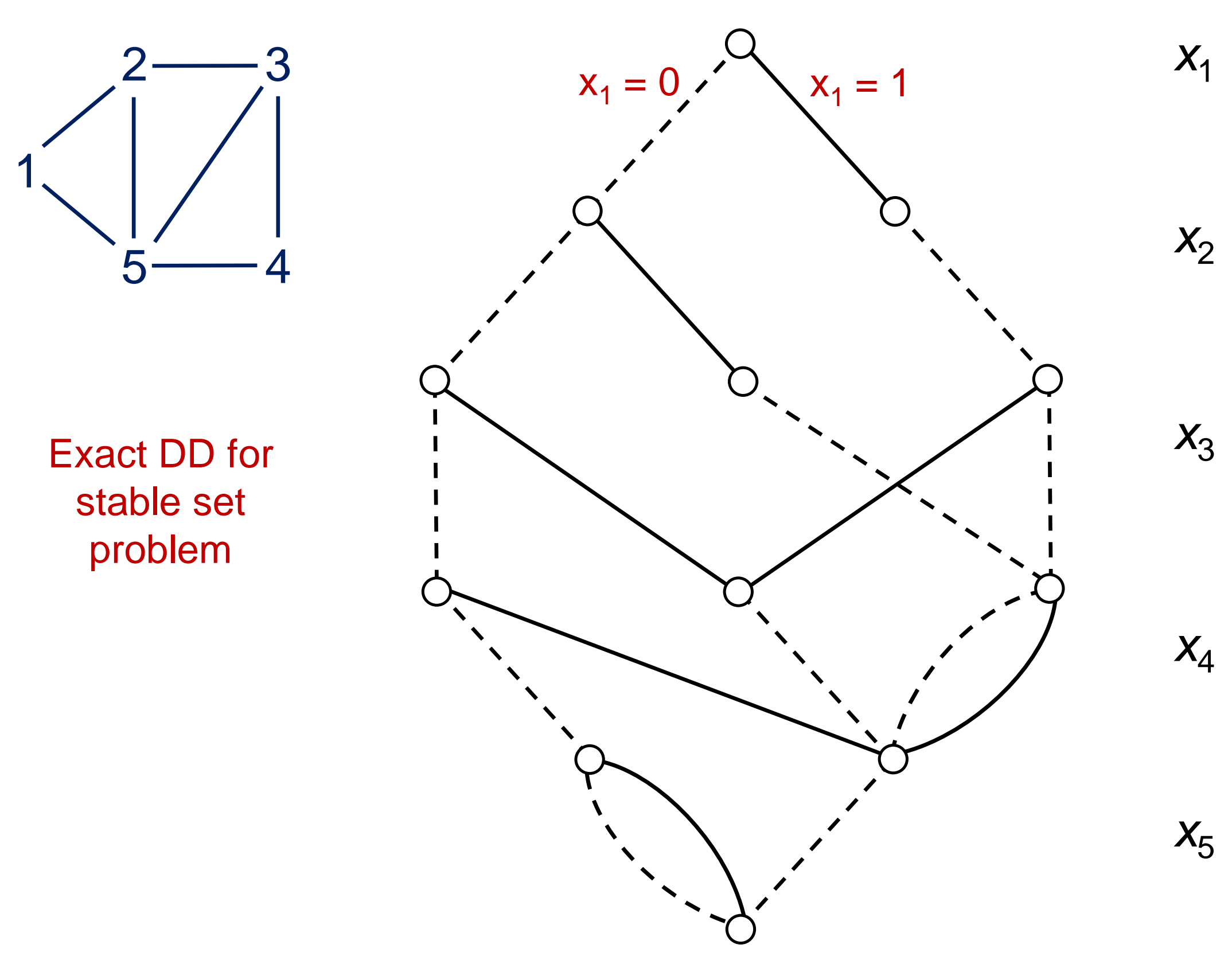

$x_{1}$

Exact DD for stable set problem 

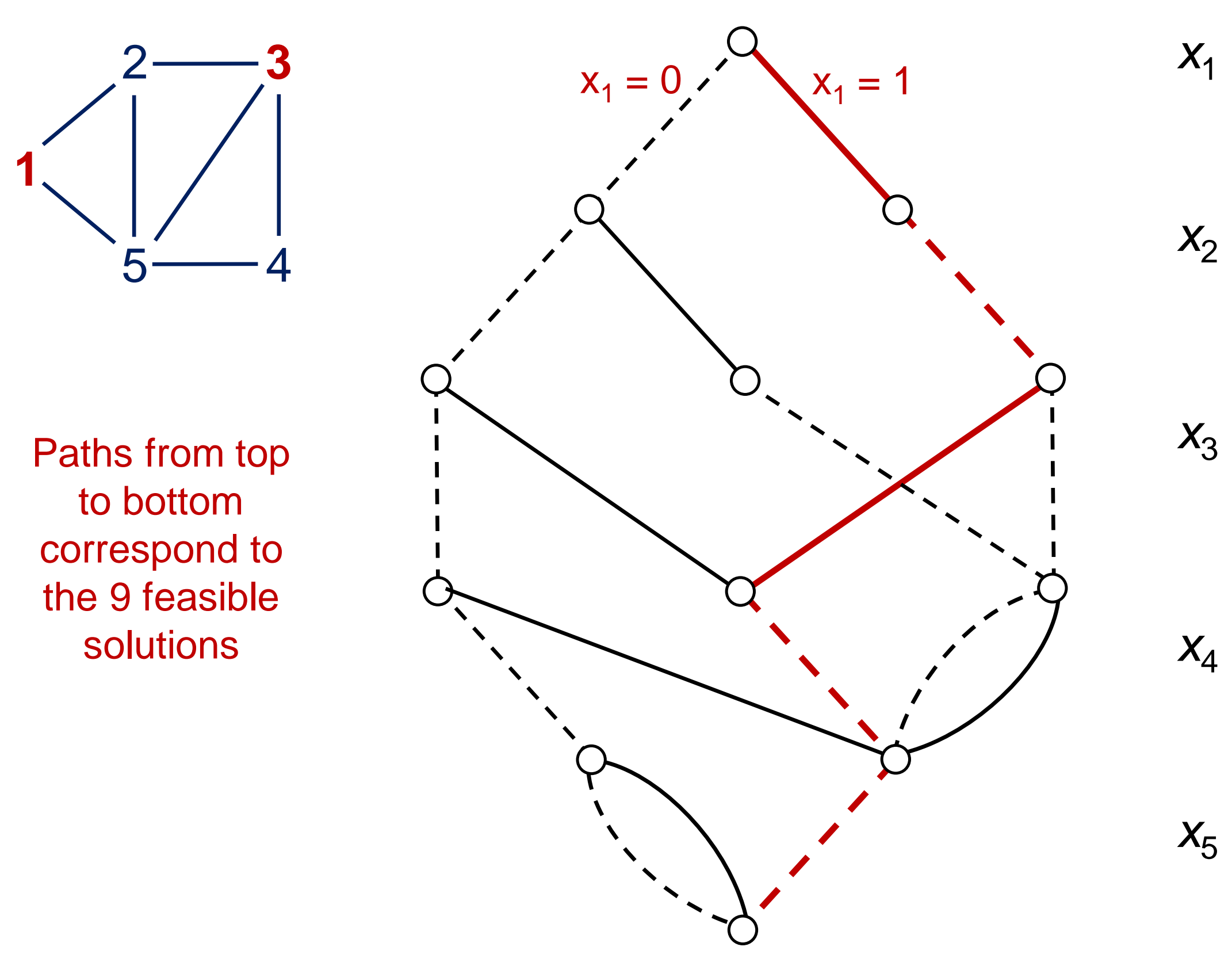

$x_{1}$

Paths from top to bottom correspond to the 9 feasible solutions 

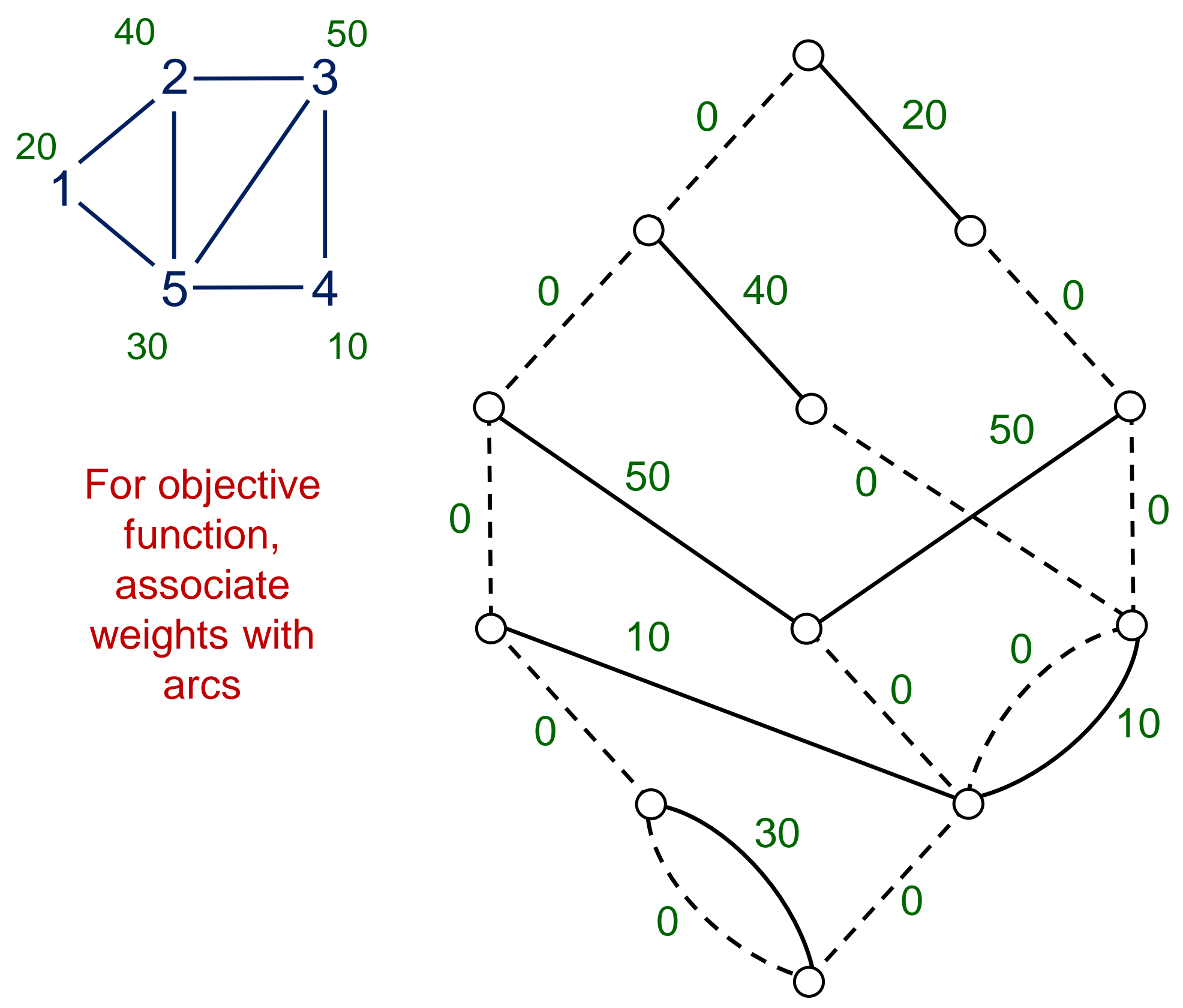

$x_{1}$

$x_{2}$

For objective function, associate weights with arcs 


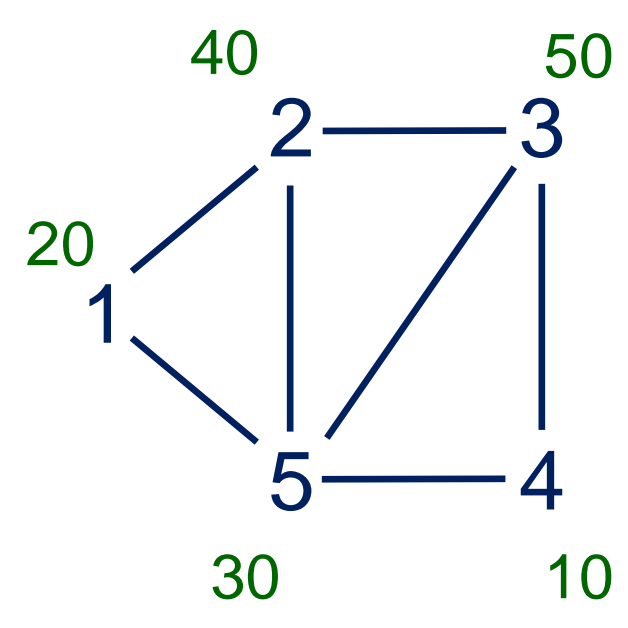

For objective function, associate weights with arcs

Optimal solution is longest path

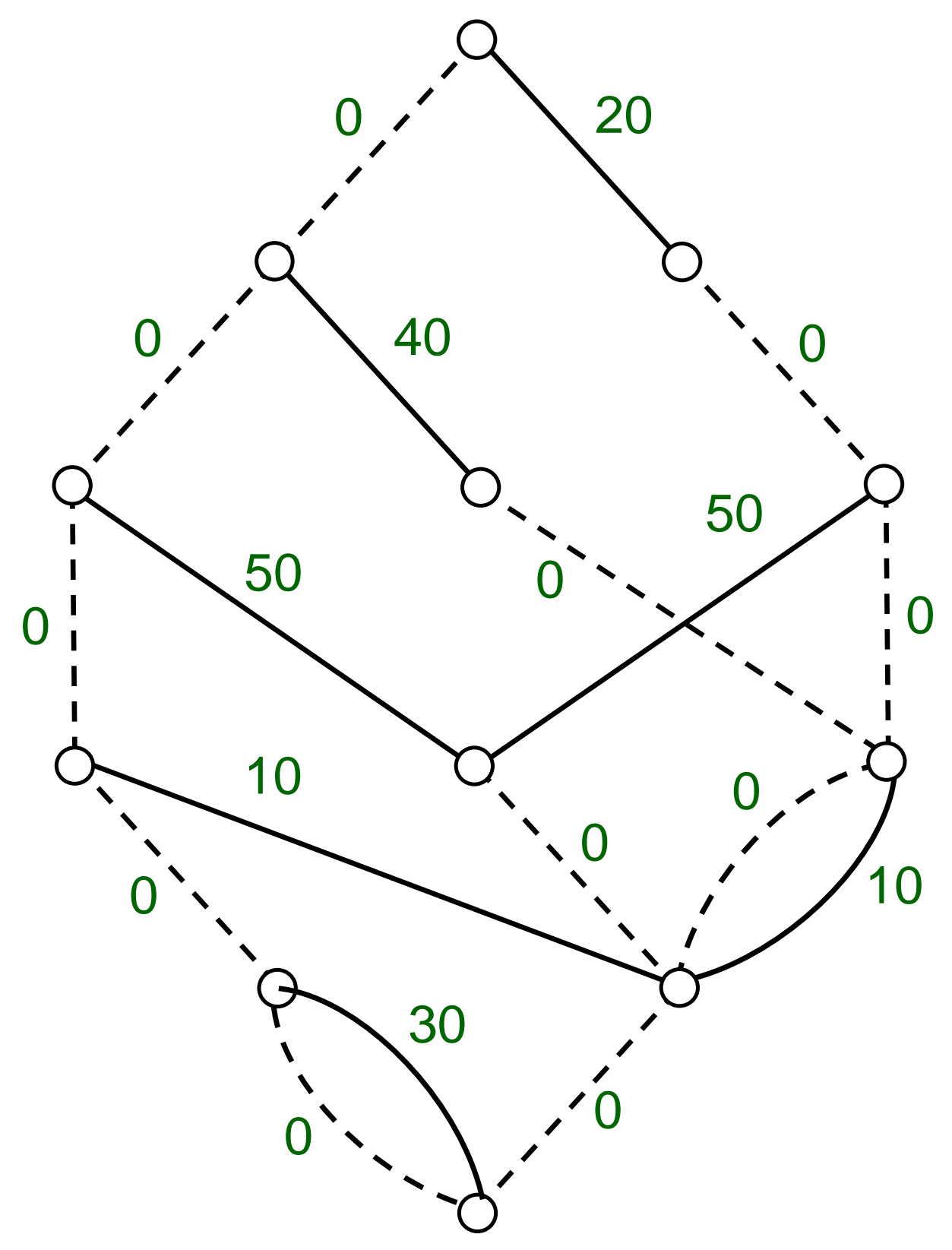

$x_{1}$

$x_{2}$

$x_{3}$

$x_{4}$

$x_{5}$ 


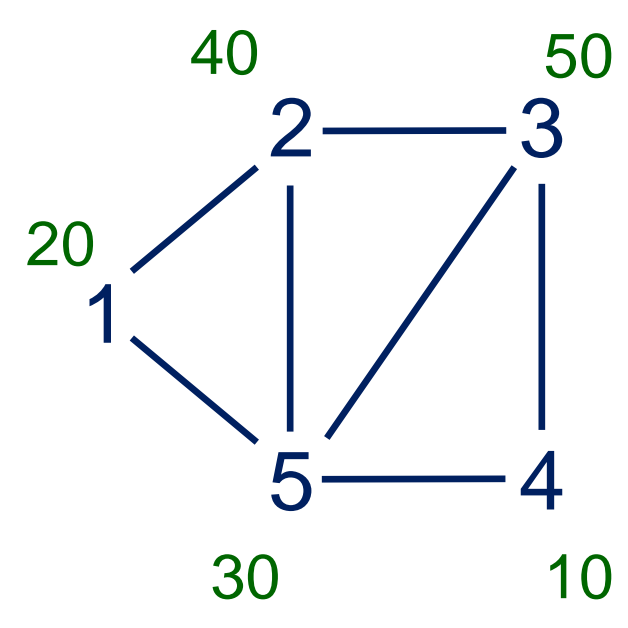

For objective function, associate weights with arcs

Optimal solution is longest path

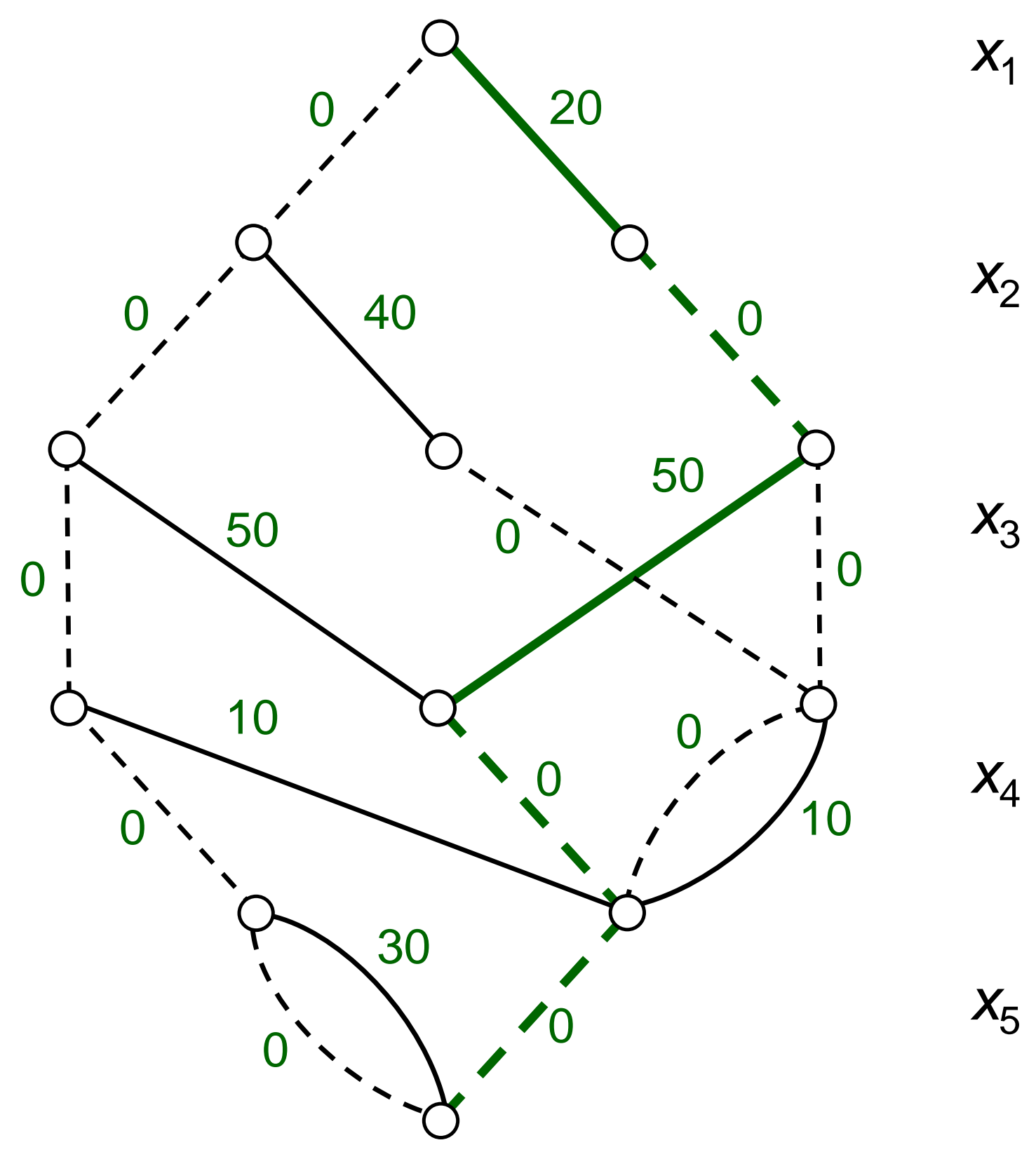




\section{Exact DD Compilation}

- Build an exact DD by associating a state with each node.

- Merge nodes with identical states. 


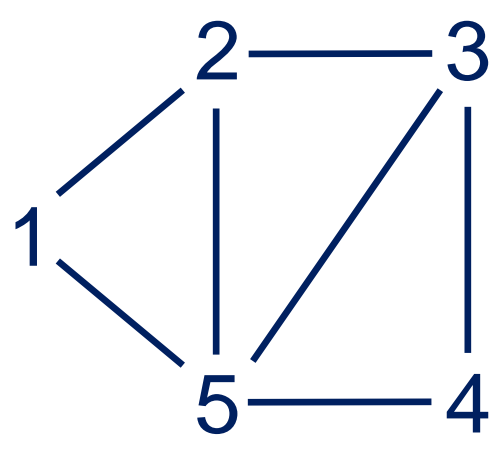

Exact DD for stable set problem

\section{To build DD,} associate state with each node

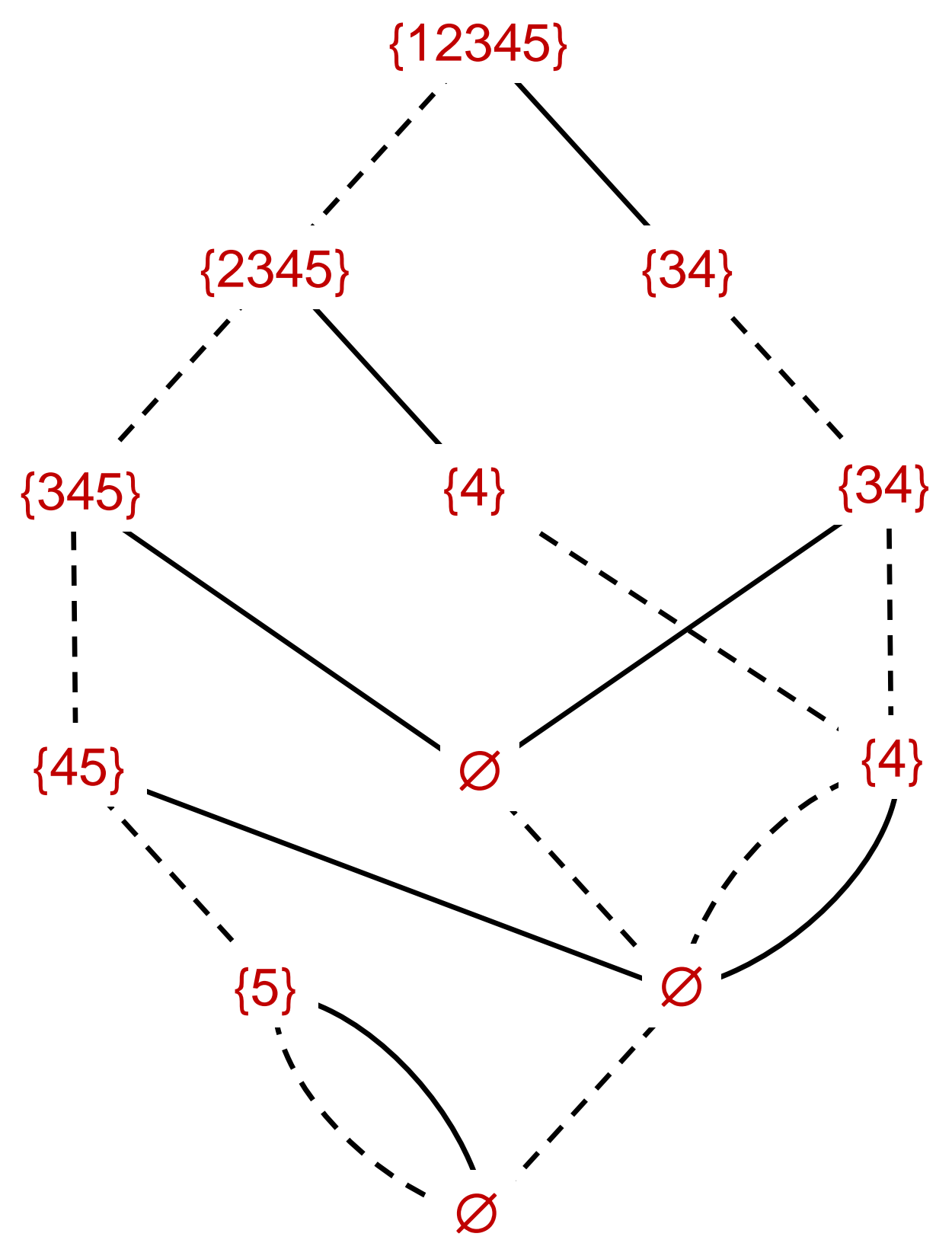

$x_{2}$

$x_{3}$

$x_{4}$

$X_{5}$ 


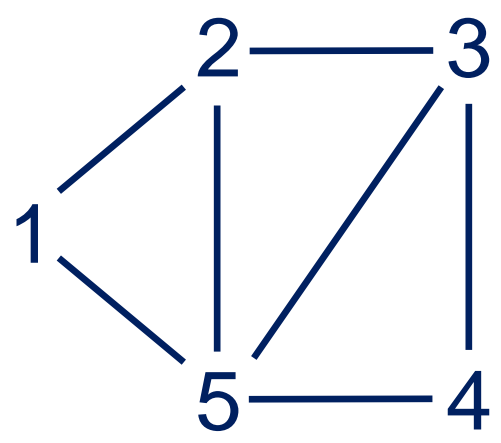
stable set problem

To build DD, associate state with each node 


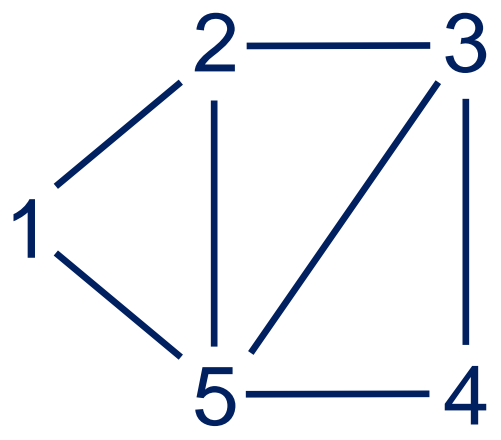

Exact DD for stable set problem

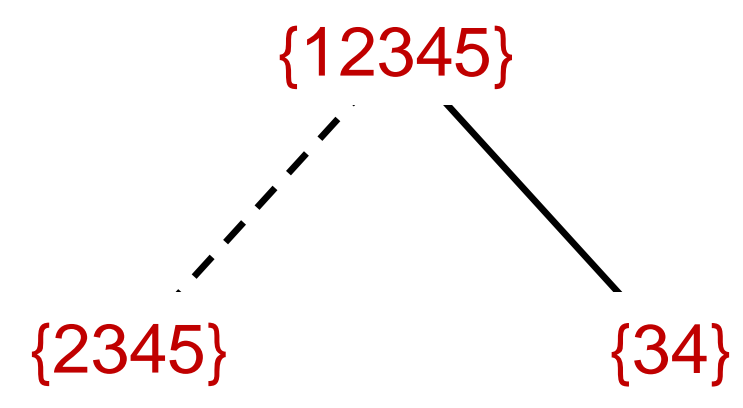

$x_{2}$

$x_{3}$

$x_{4}$

To build DD, associate state with each node 

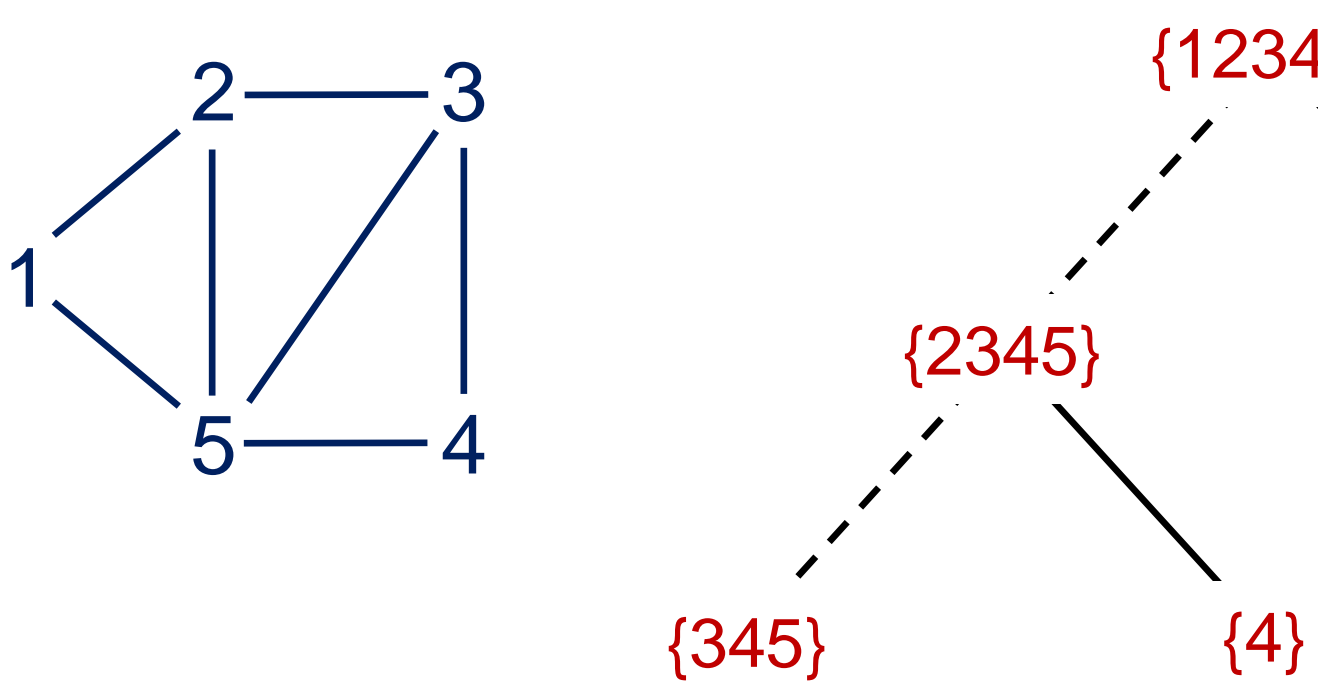

$x_{1}$

$x_{2}$

$\{34\}$

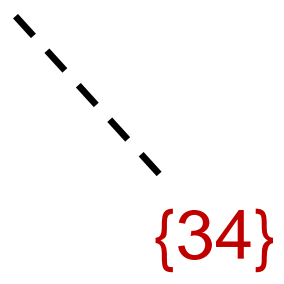

Exact DD for

$x_{3}$ stable set problem

$x_{4}$

To build DD, associate state with each node 


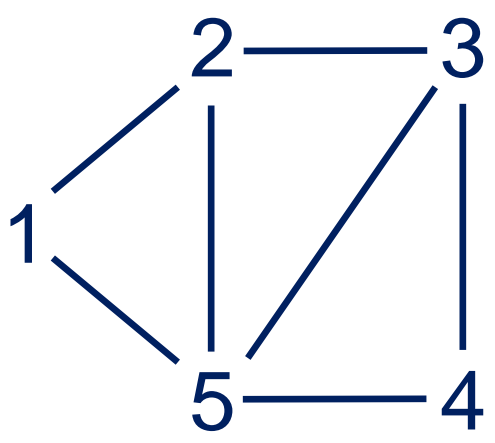

Exact DD for stable set problem

Merge nodes that correspond to the same state

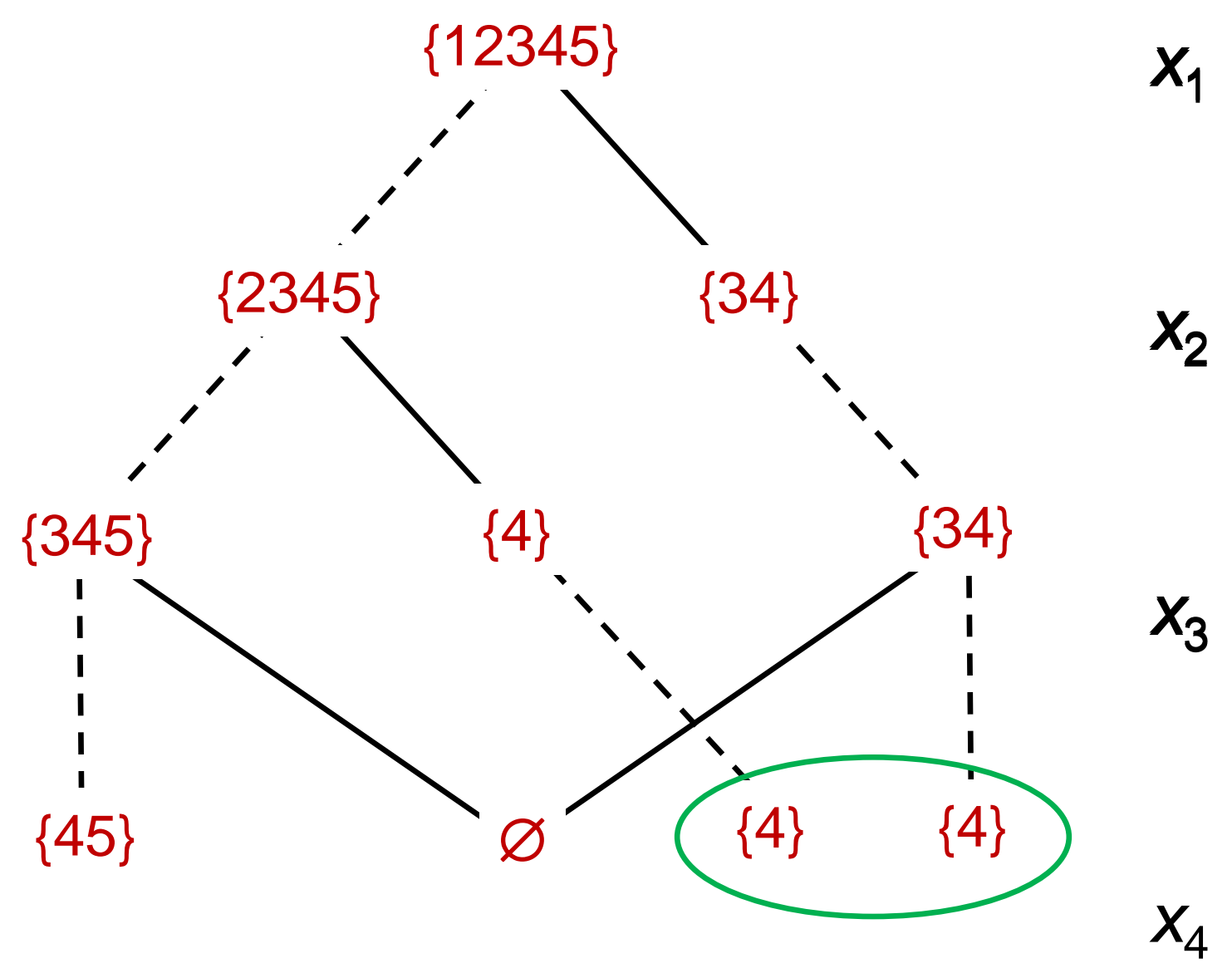

$x_{5}$ 


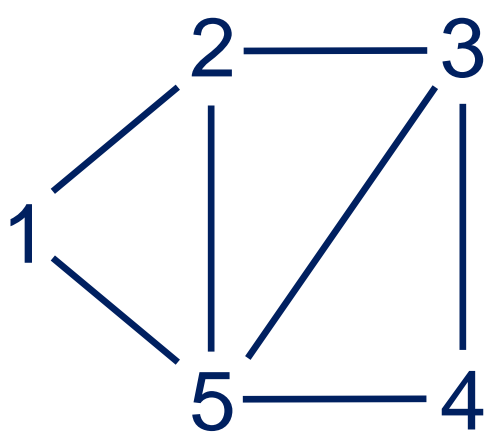

Exact DD for stable set problem

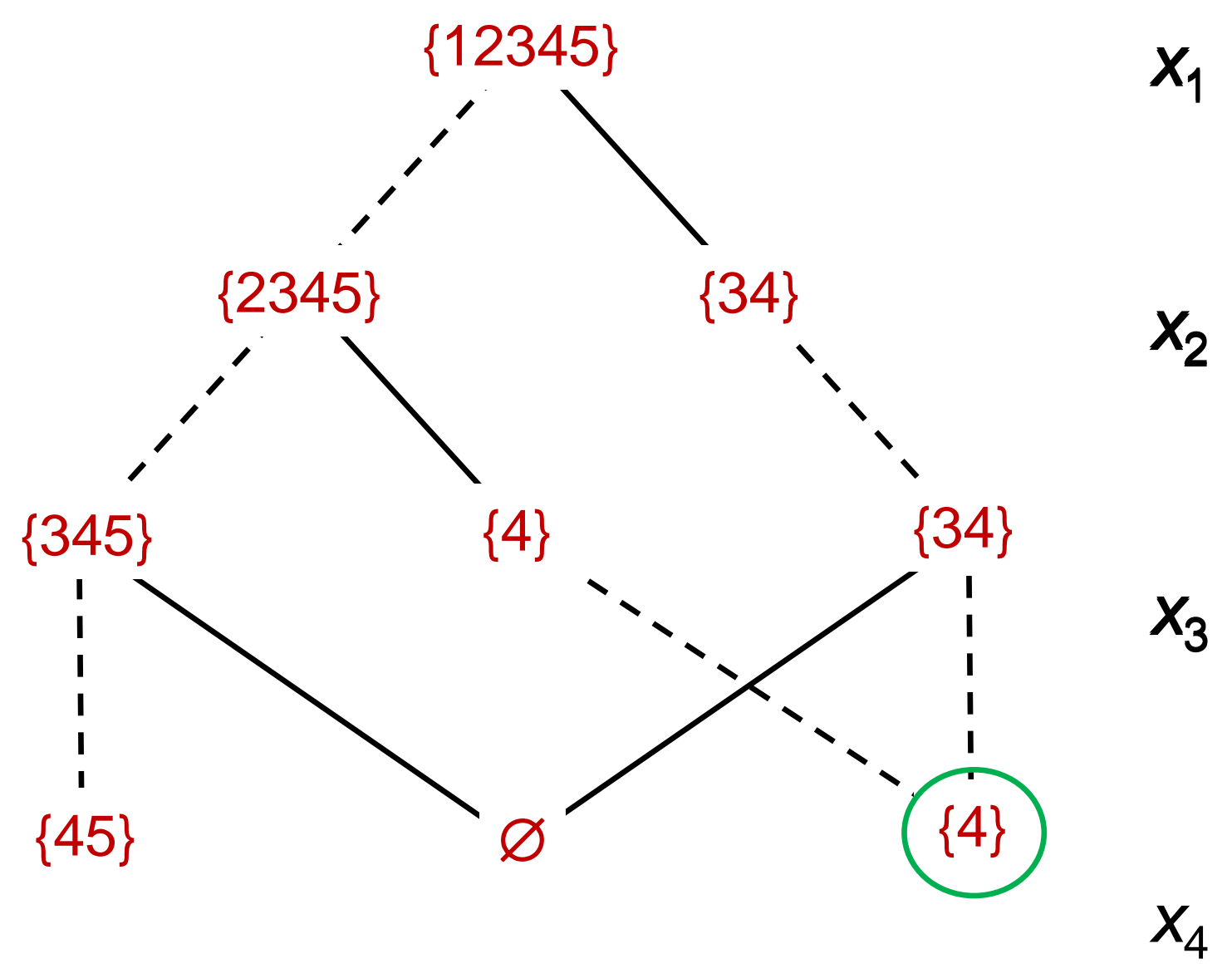

Merge nodes that correspond to the same state $x_{5}$ 


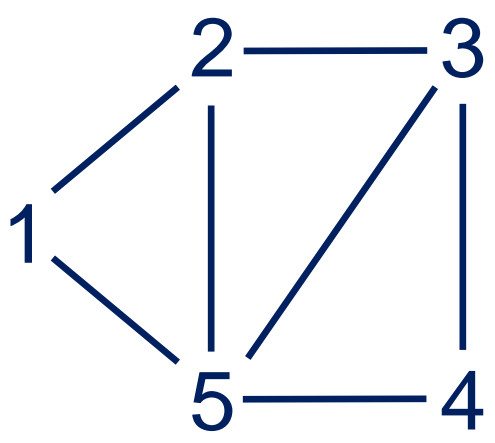

Exact DD for stable set problem

\section{To build DD,} associate state with each node

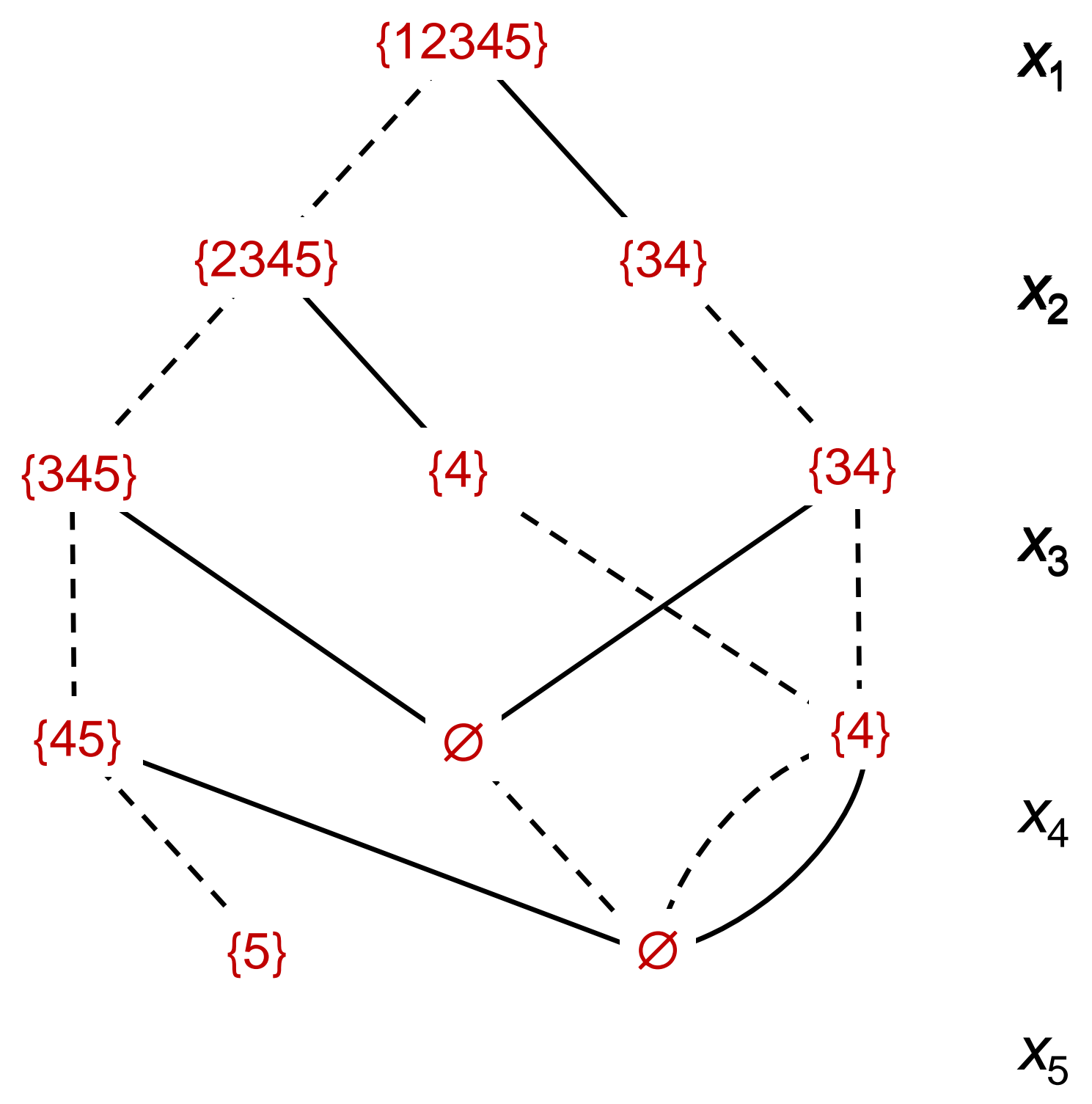

$x_{2}$

$x_{3}$

$x_{5}$ 


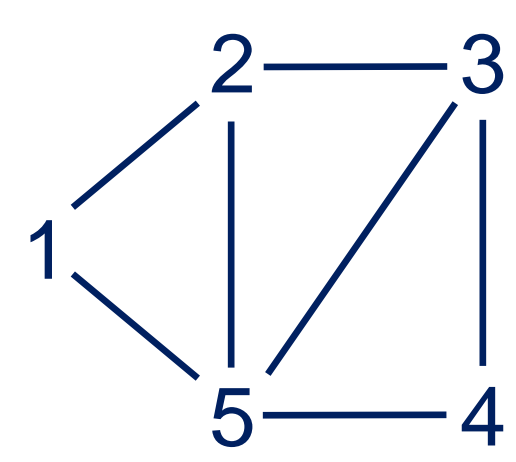

Exact DD for stable set problem

Resulting DD is not necessarily reduced

(it is in this case).

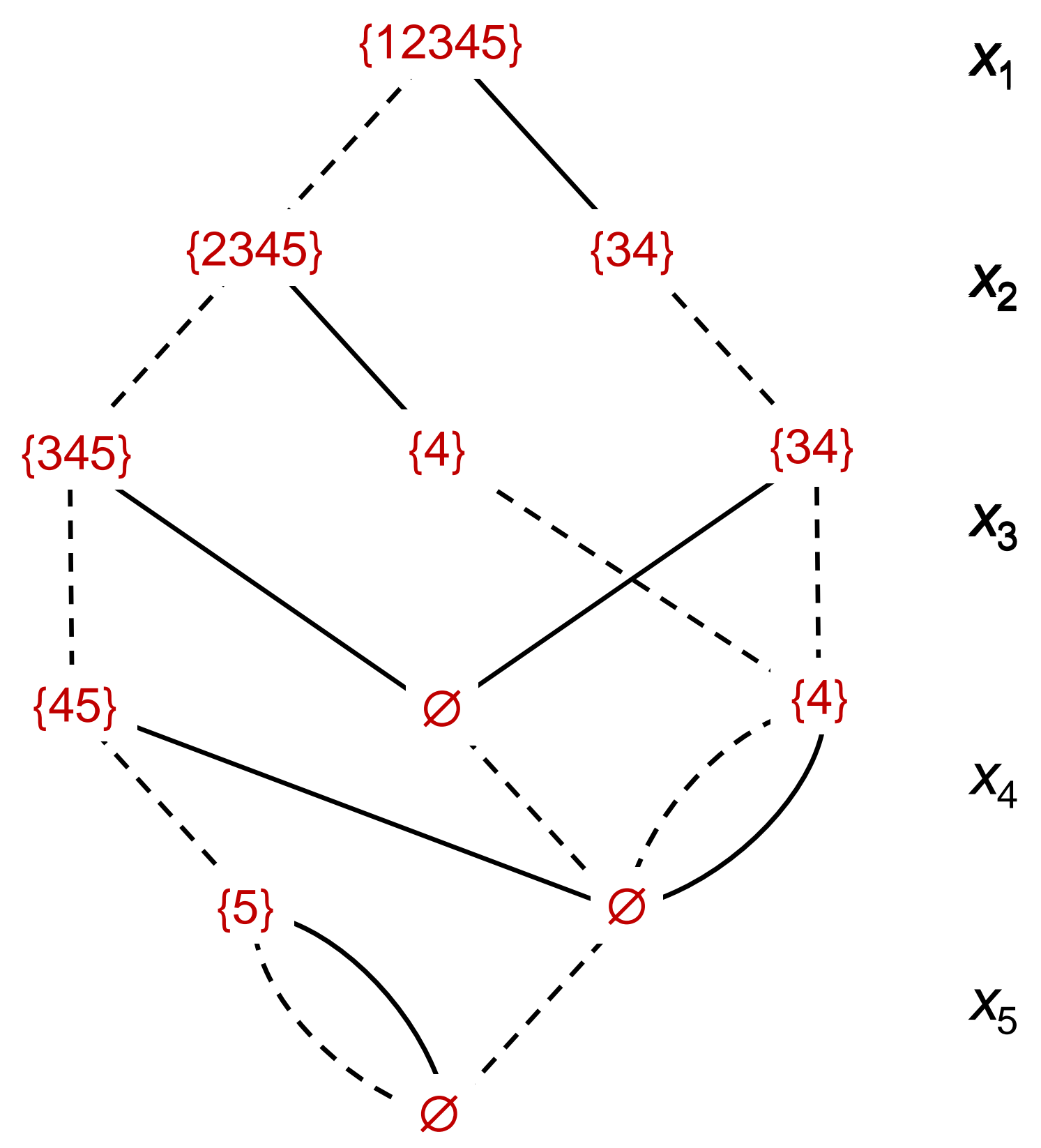

$x_{1}$

$x_{2}$

$x_{3}$

$x_{4}$

$x_{5}$ 


\section{A General-Purpose Solver}

- The decision diagram tends to grow exponentially.

- To build a practical solver:

- Use limited-width relaxed decision diagrams to bound the objective value.

- Use limited-width restricted decision diagrams for primal heuristic

- Use a recursive dynamic programming model.

- Use novel branching scheme within relaxed decision diagrams. 


\section{Relaxed Decision Diagrams}

- A relaxed DD represents a superset of feasible set.

- Shortest (longest) path length is a bound on optimal value.

- Size of DD is controlled.

- Analogous to LP relaxation in IP, but discrete.

- Does not require linearity, convexity, or inequality constraints. 


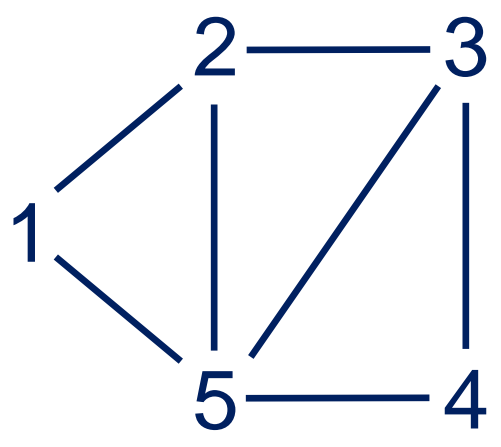

$\{12345\}$

$x_{1}$

$x_{2}$

$x_{3}$

To build relaxed

$\mathrm{DD}$, merge

some additional

$x_{4}$

nodes as we go along

$x_{5}$ 


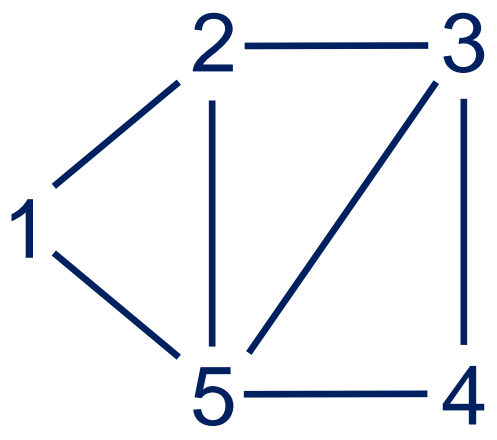

$\{2345\}$

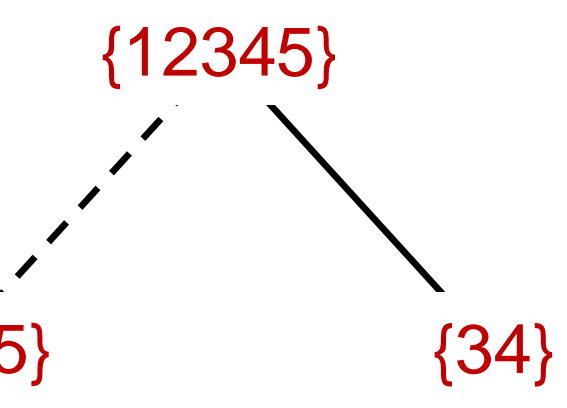

$\{34\}$

$x_{3}$

To build relaxed

DD, merge

some additional

nodes as we go along 

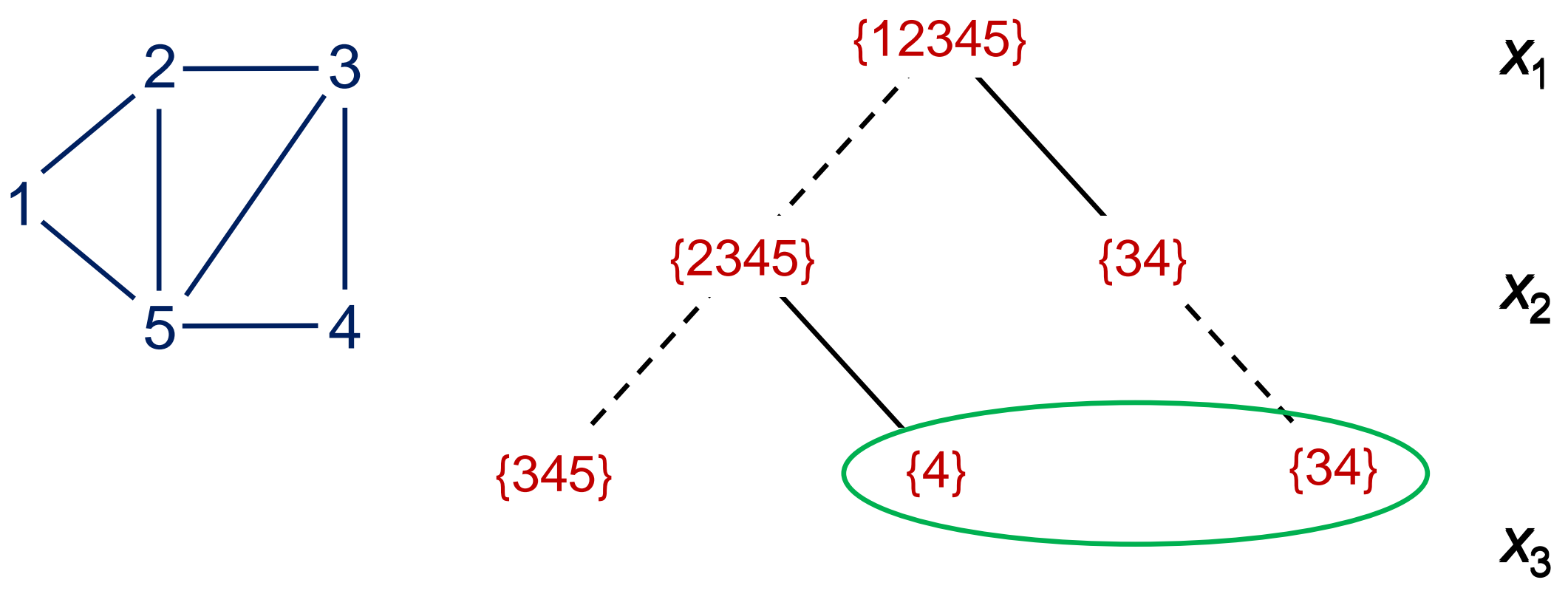

To build relaxed

DD, merge

some additional

nodes as we go along.

Take the union $x_{5}$ of merged states 

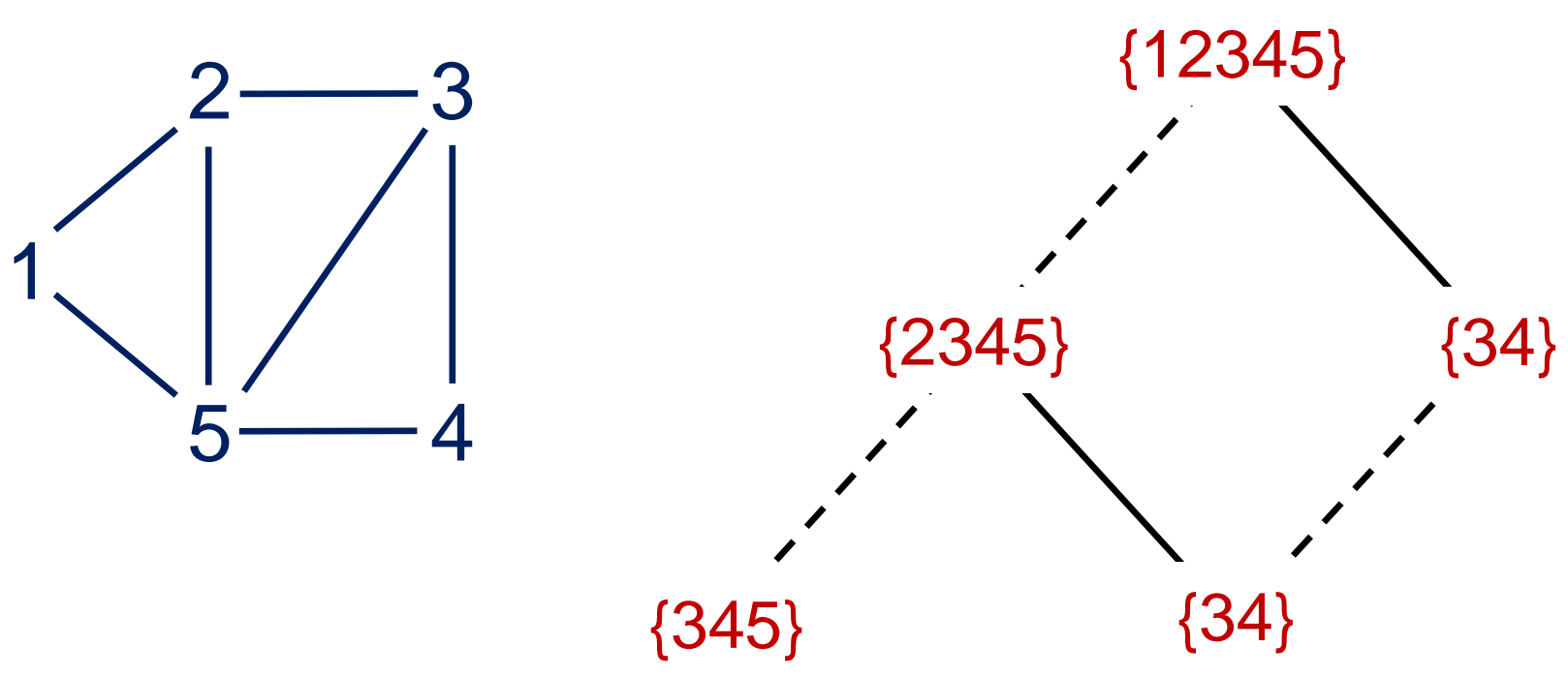

$x_{1}$

$x_{2}$

$x_{3}$

To build relaxed

DD, merge

some additional

$x_{4}$

nodes as we go

along.

Take the union

$x_{5}$

of merged

states. 

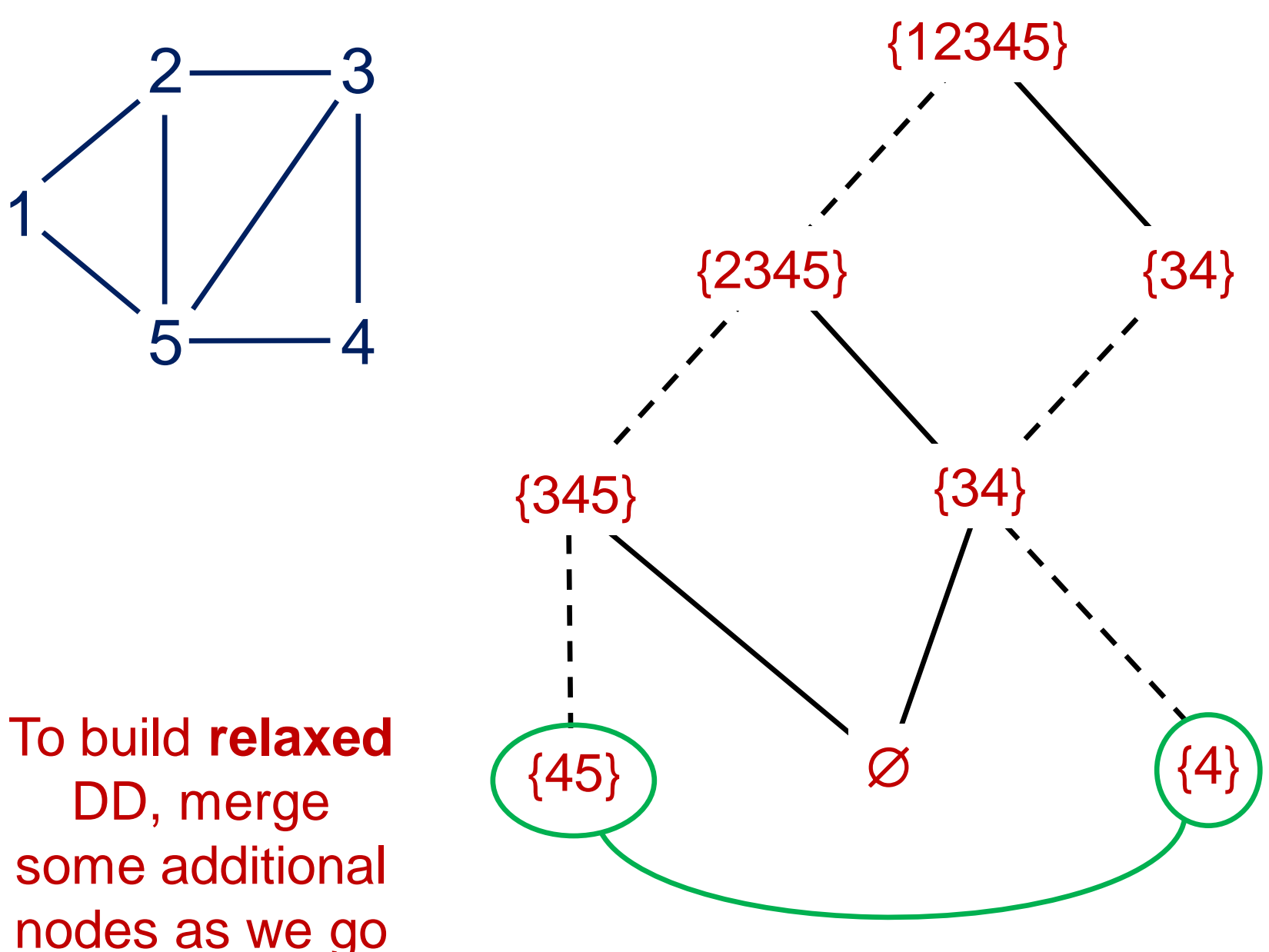

$x_{1}$

$x_{2}$

To build relaxed DD, merge some additional nodes as we go along.

Take the union $x_{5}$ of merged states. 

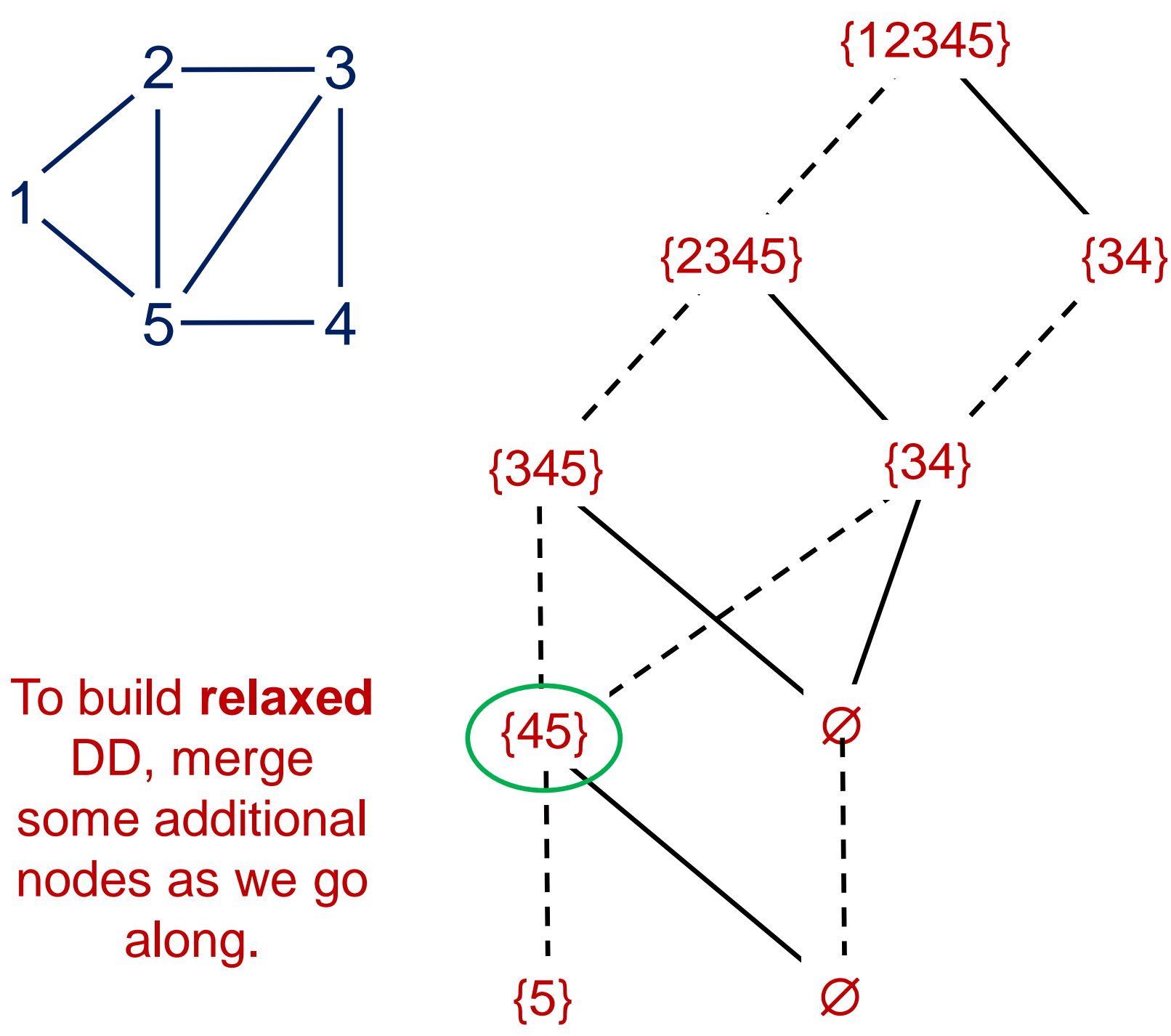

$x_{1}$

$x_{2}$

To build relaxed DD, merge some additional nodes as we go along.

Take the union of merged states. 


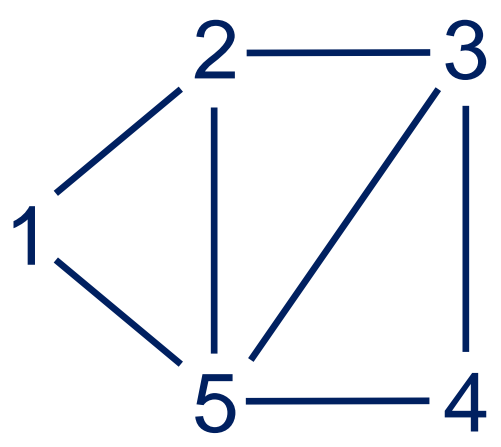

Width $=2$

Represents 11 solutions, including 9 feasible solutions

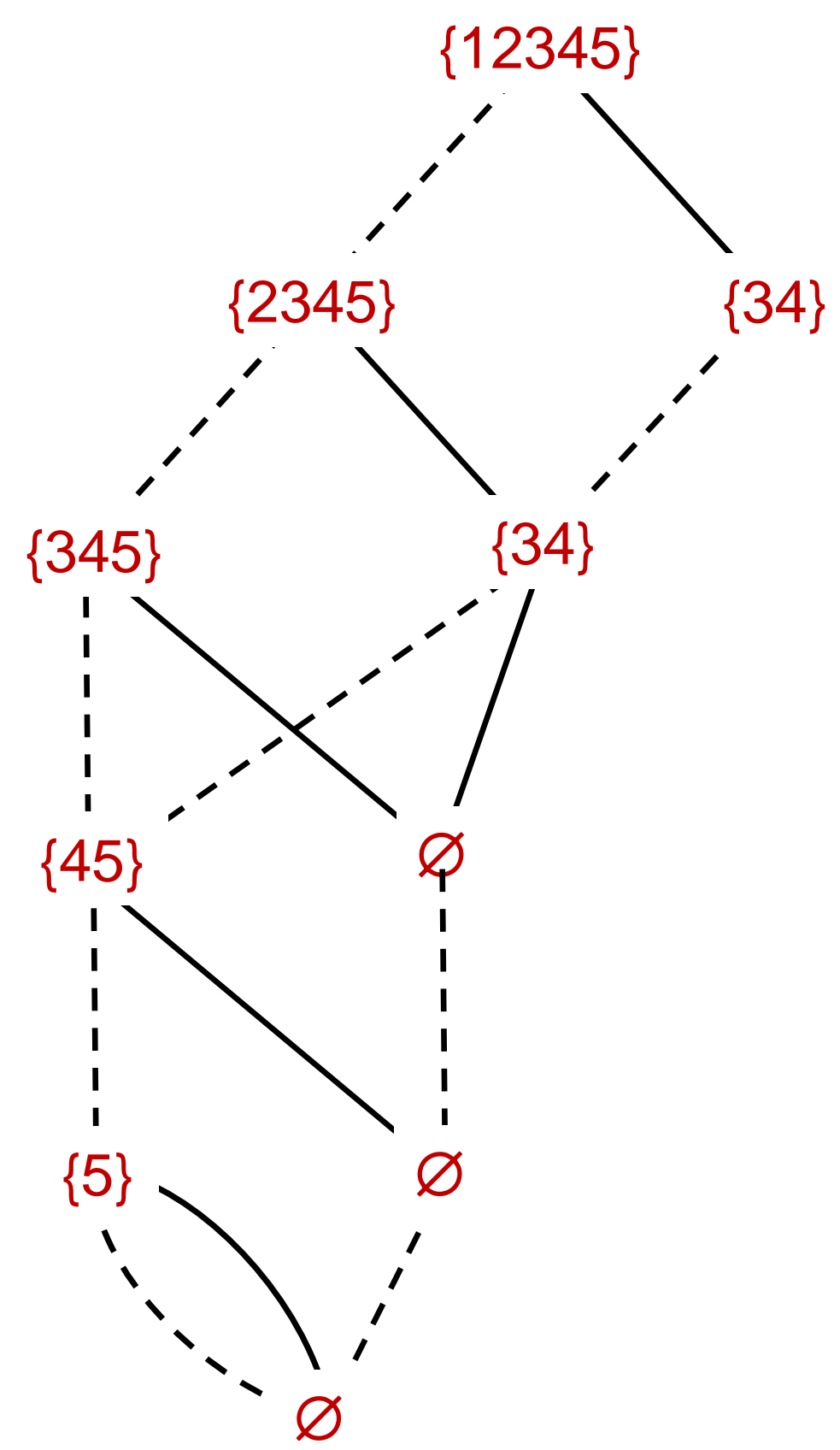




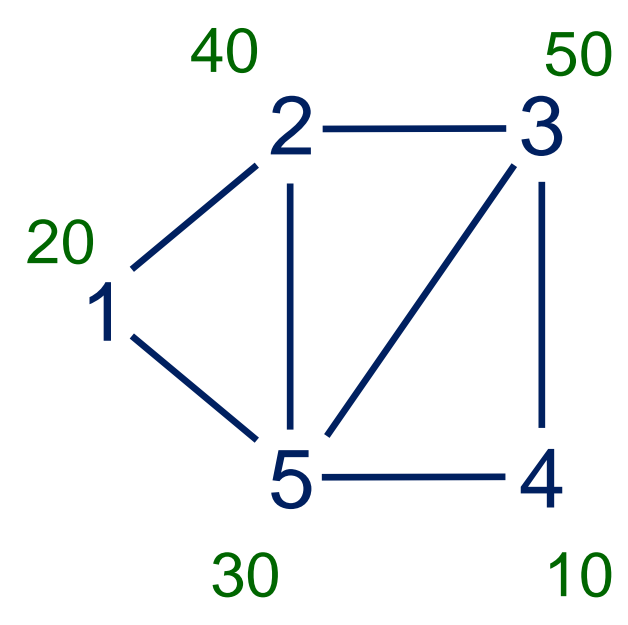

$x_{1}$

$x_{2}$

Width $=2$

Represents

11 solutions, including 9 feasible solutions

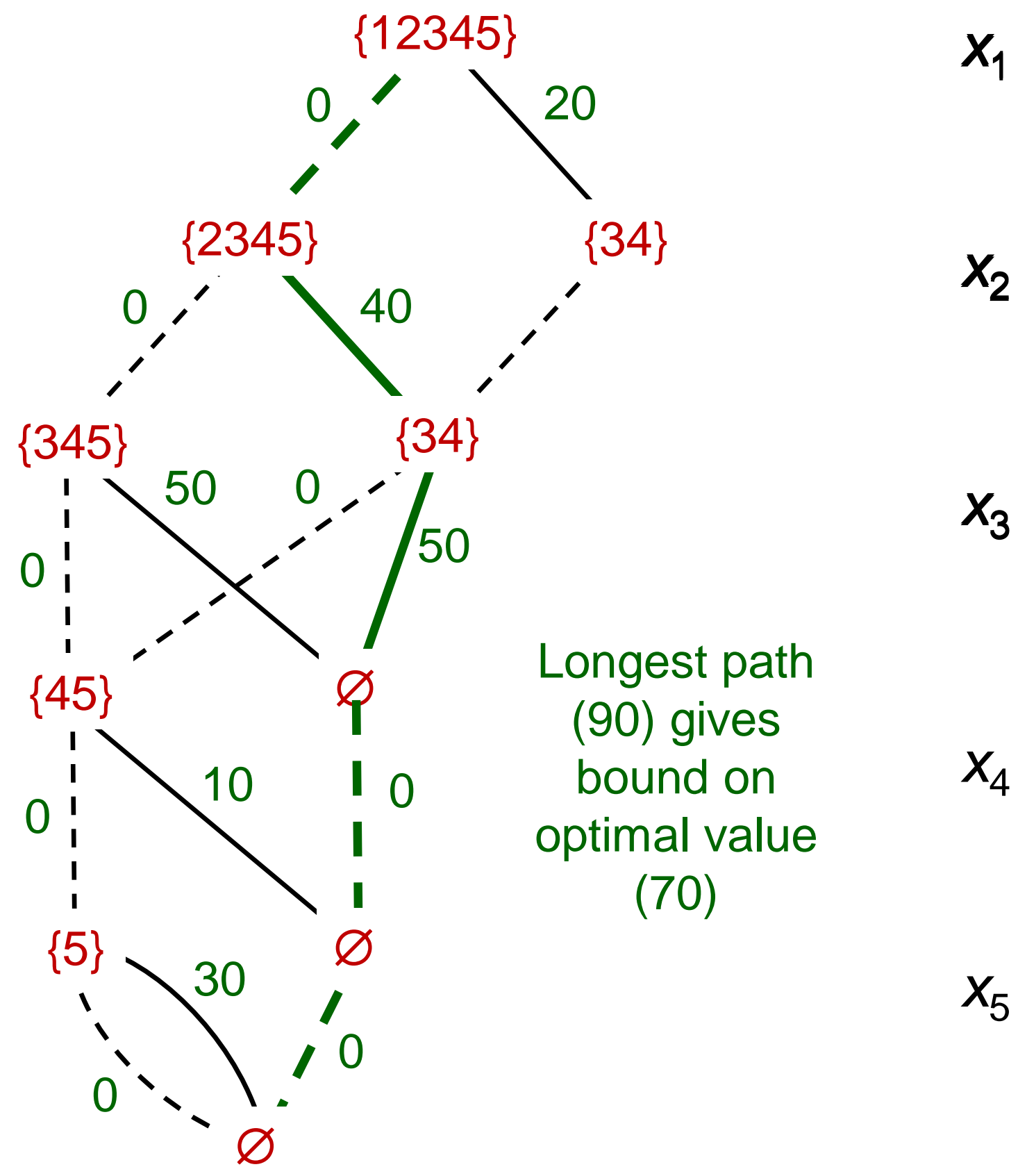




\section{Relaxed Decision Diagrams}

- Alternate relaxation method: node refinement.

- Start with DD of width 1 representing Cartesian product of variable domains.

- Split nodes so as to remove some infeasible paths.

- Will be illustrated in next tutorial. 


\section{Relaxed Decision Diagrams}

- Original application: enhanced propagation in constraint programming

- In multiple alldiff problem (graph coloring), reduced 1 million node search trees to 1 node. 


\section{Relaxed Decision Diagrams}

- Wider diagrams yield tighter bounds

- But take longer to build.

- Adjust width dynamically.

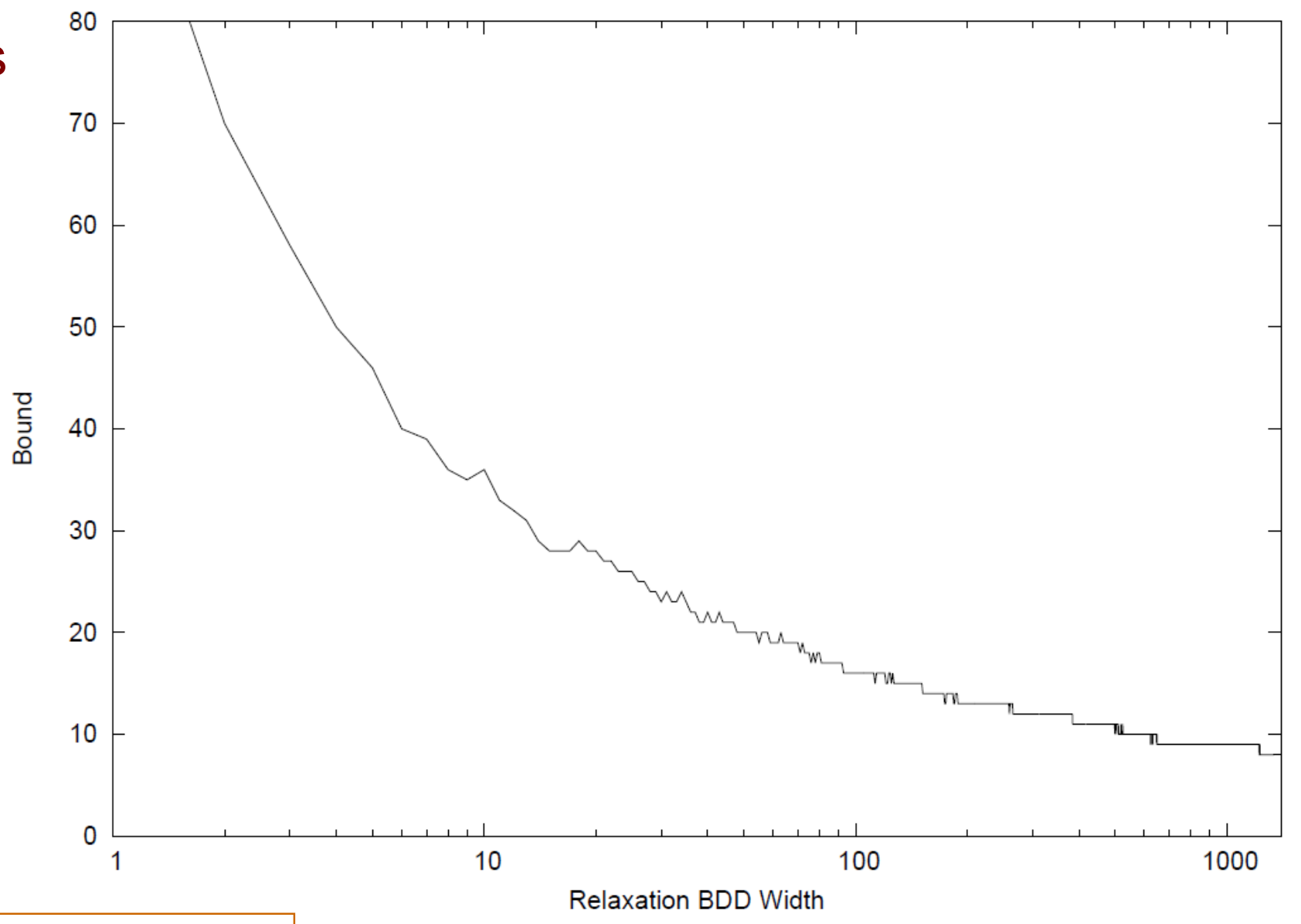




\section{Relaxed Decision Diagrams}

- DDs vs. CPLEX bound at root node for max stable set problem

- Using CPLEX default cut generation

- DD max width of 1000.

- DDs require about $5 \%$ the time of CPLEX

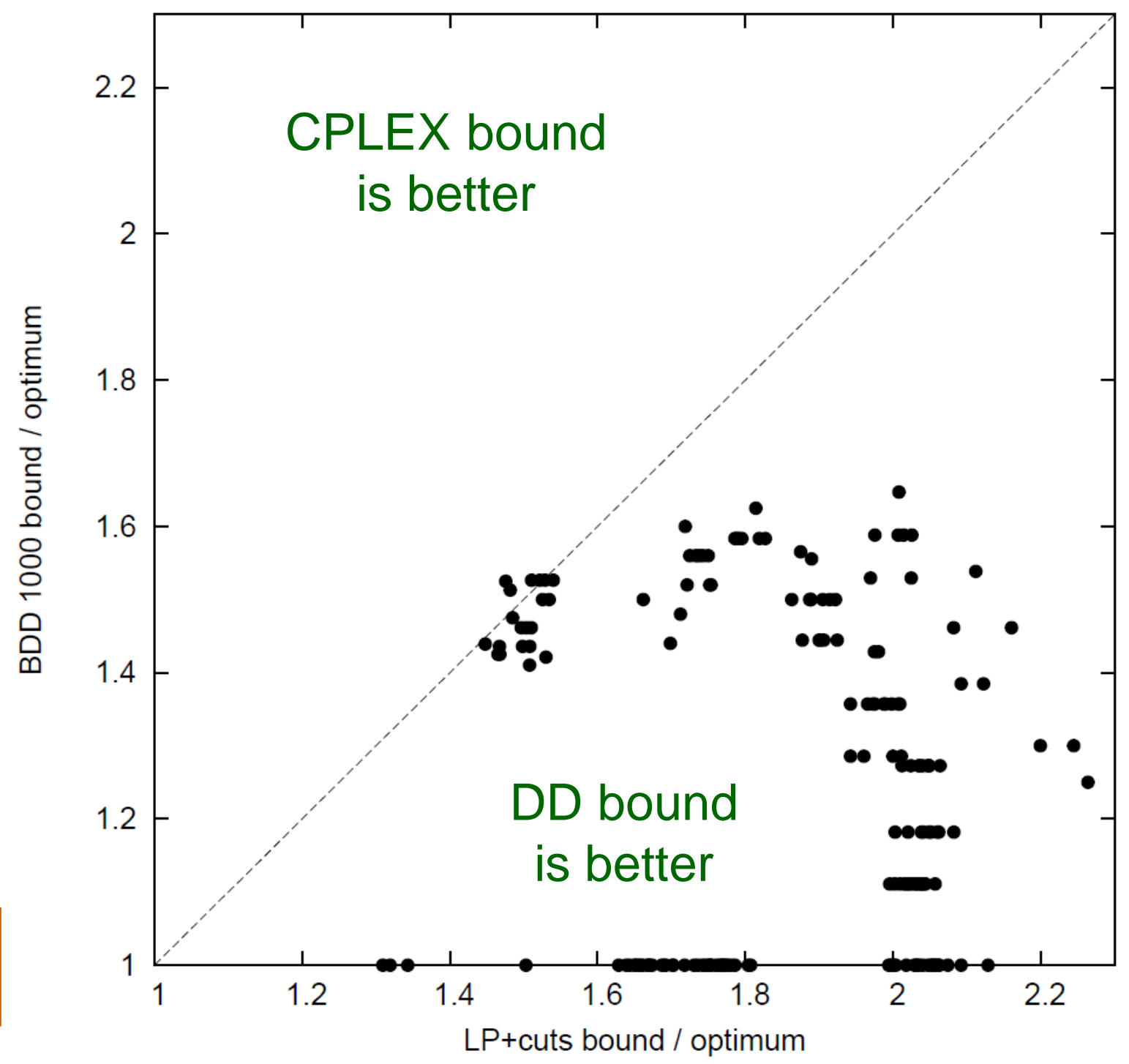




\section{Restricted Decision Diagrams}

- A restricted DD represents a subset of the feasible set.

- Restricted DDs provide a basis for a primal heuristic.

- Shortest (longest) paths in the restricted DD provide good feasible solutions.

- Generate a limited-width restricted DD by deleting nodes that appear unpromising. 
Set covering problem

$$
\begin{aligned}
& x_{1}+x_{2}+x_{3} \geq 1 \\
& x_{1}+x_{4}+x_{5} \geq 1 \\
& x_{2}+x_{4}+x_{6} \geq 1
\end{aligned}
$$

Sets

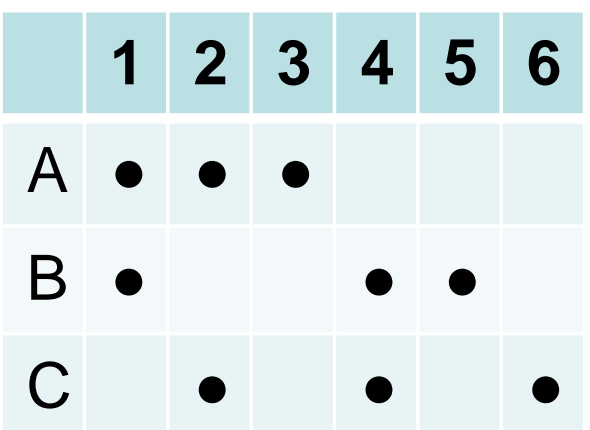

52 feasible solutions.

Minimum cover of 2, e.g. $x_{1}, x_{2}$ 
Restricted DD of width 4

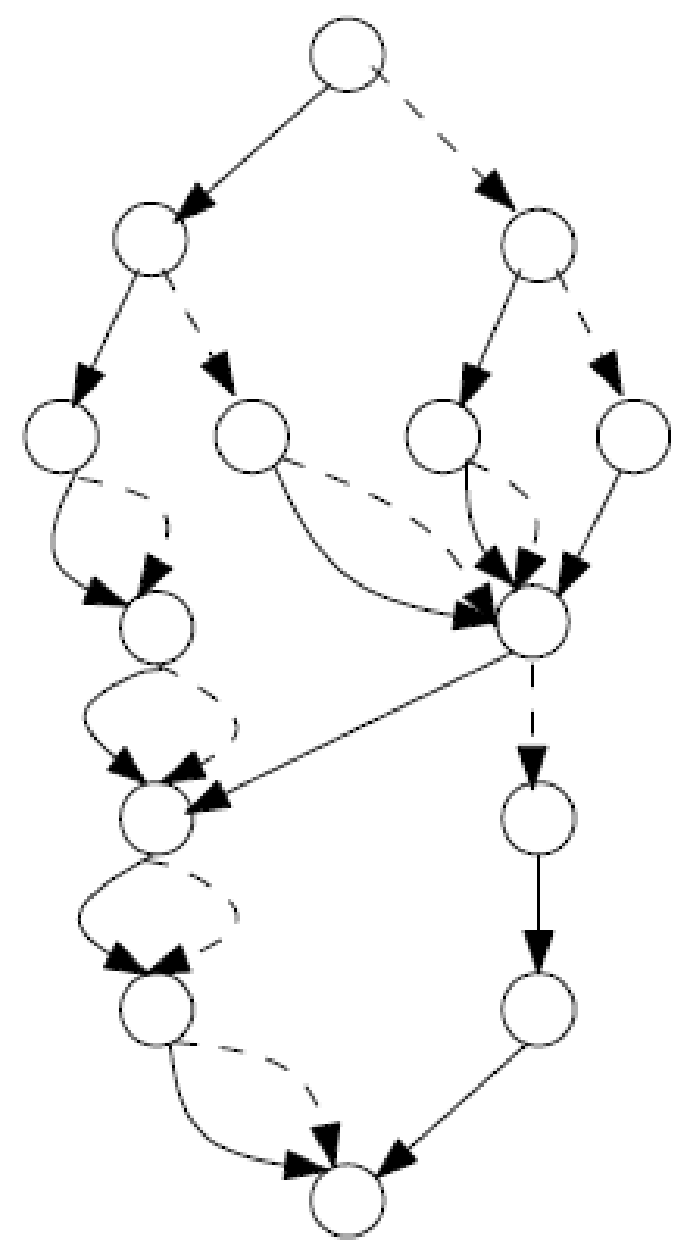

Several shortest paths have length 2.

All are minimum covers.

41 paths ( $<52$ feasible solutions) 
Restricted DD of width 4

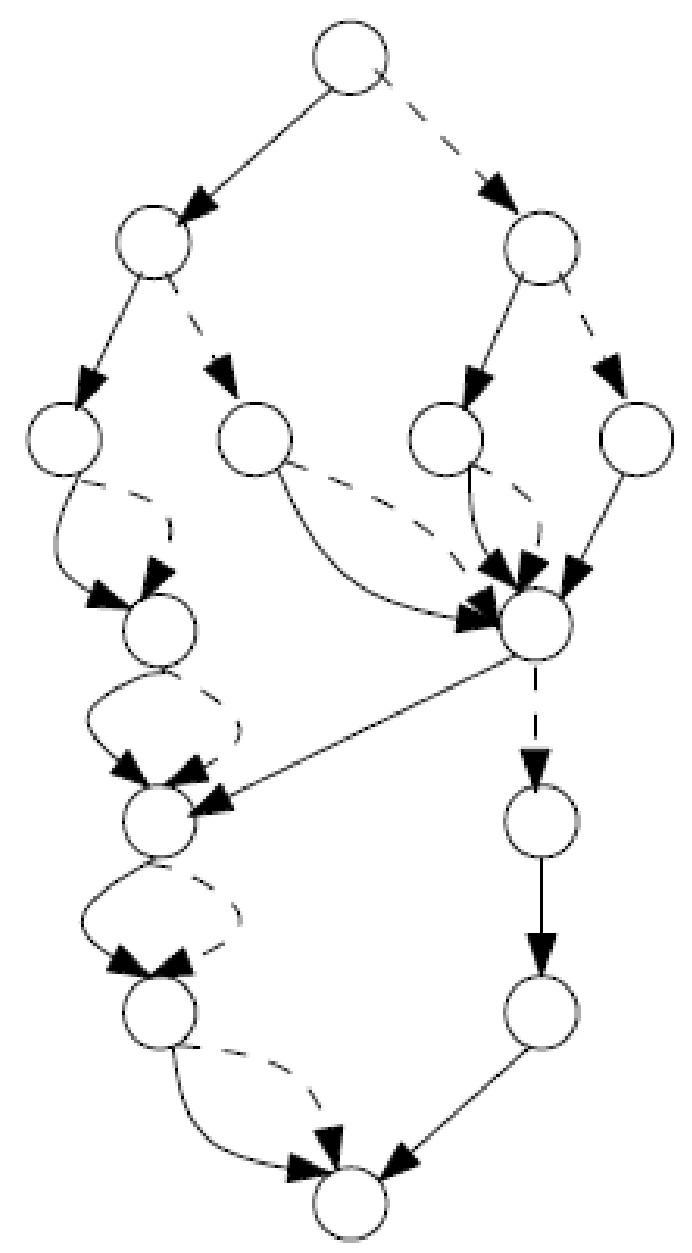

Several shortest paths have length 2.

All are minimum covers.

In this case, restricted DD delivers optimal solutions.

41 paths (< $<2$ feasible solutions) 
Optimality gap for set covering, $n$ variables

Restricted DDs vs

Primal heuristic at root node of CPLEX

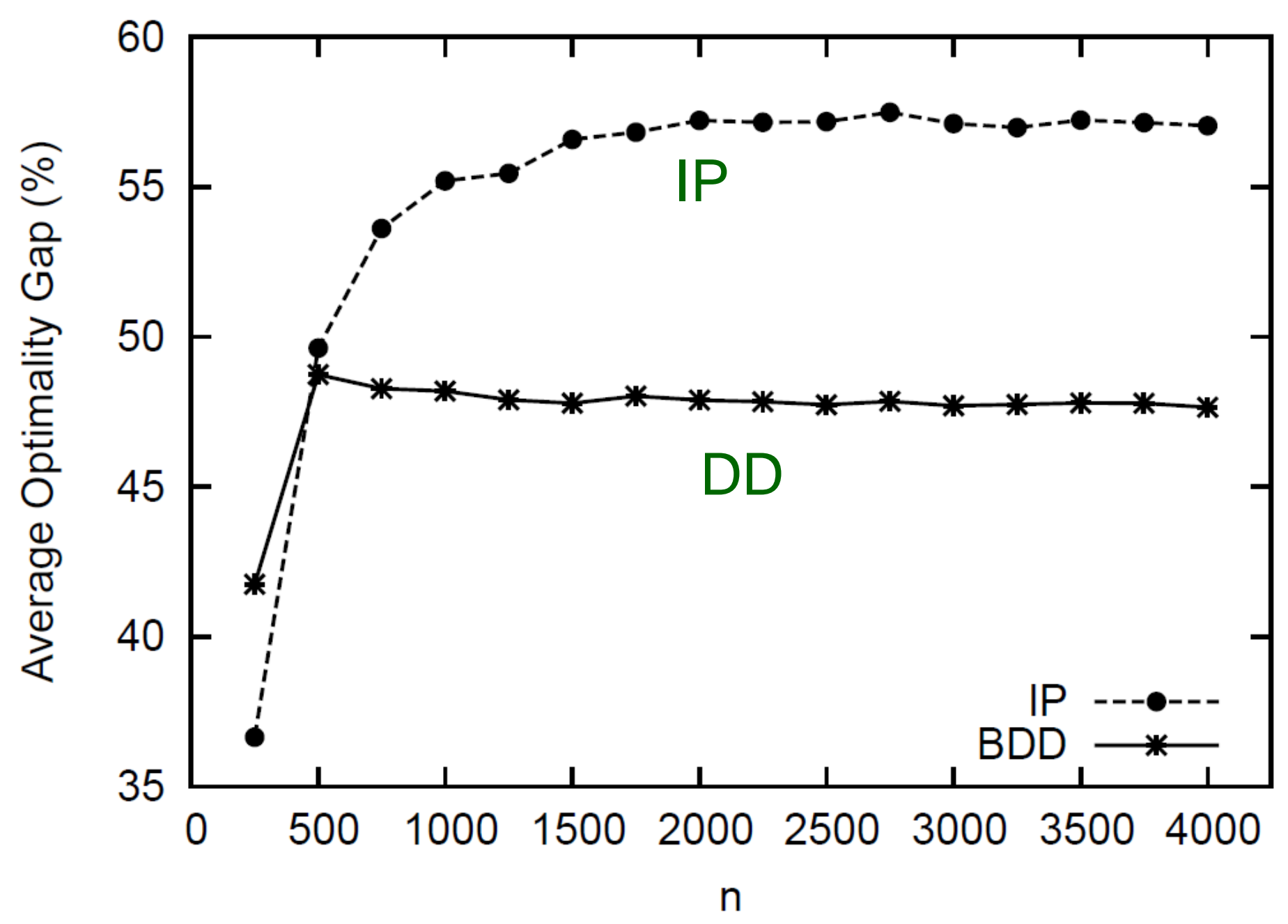




\section{Computation time}
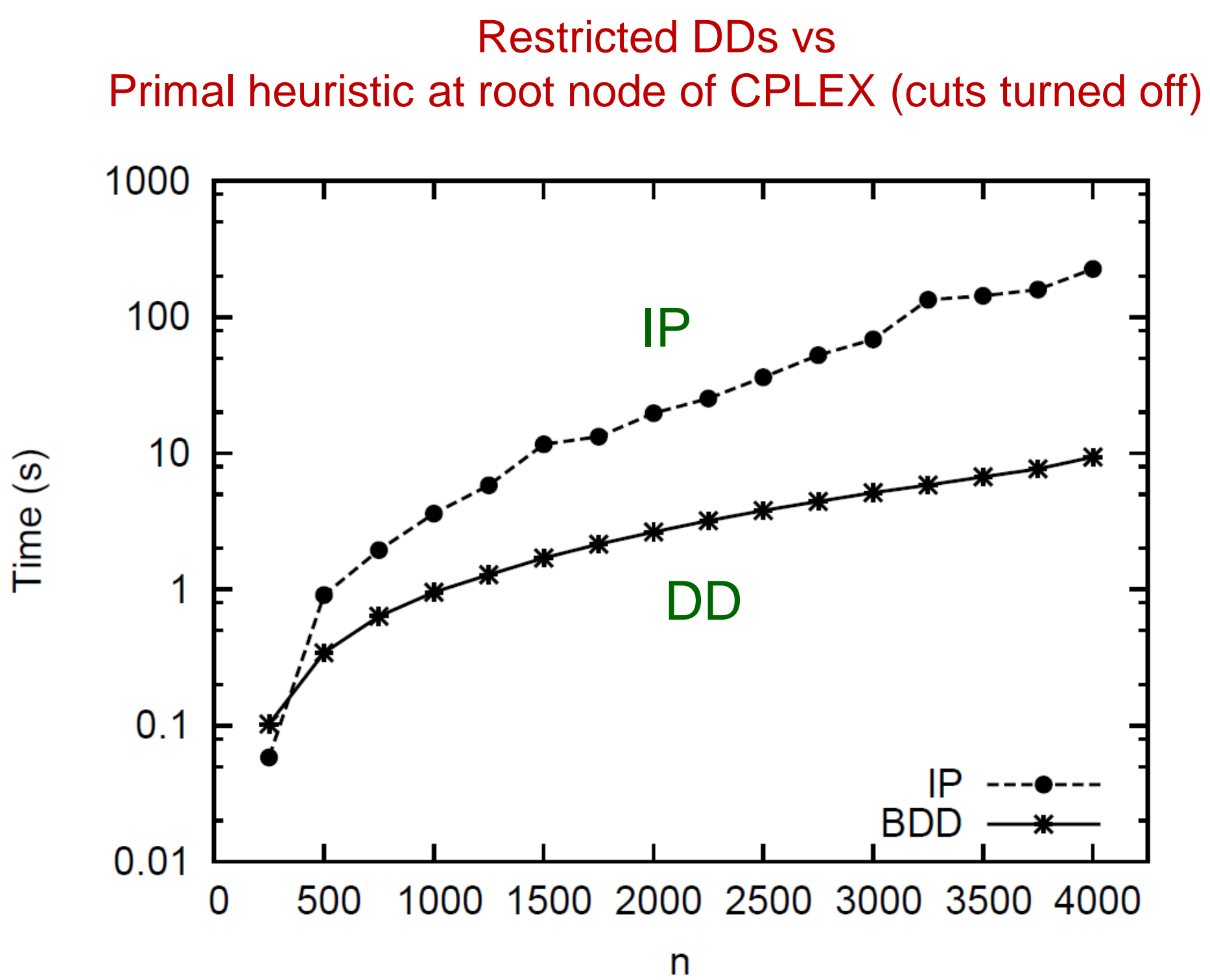


\section{Dynamic Programming Model}

- Formulate problem with dynamic programming model.

- Rather than constraint set.

- Problem must have recursive structure

- But there is great flexibility to represent constraints and objective function.

- Any function of current state is permissible.

- We don't care if state space is exponential, because we don't solve the problem by dynamic programming. 


\section{Dynamic Programming Model}

- Formulate problem with dynamic programming model.

- Rather than constraint set.

- Problem must have recursive structure

- But there is great flexibility to represent constraints and objective function.

- Any function of current state is permissible.

- We don't care if state space is exponential, because we don't solve the problem by dynamic programming.

- State variables are the same as in relaxed DD.

- Must also specify state merger rule. 


\section{Dynamic Programming Model}

- Max stable set problem on a graph.

- State $=$ set of vertices that can be added to stable set.

Recursion:

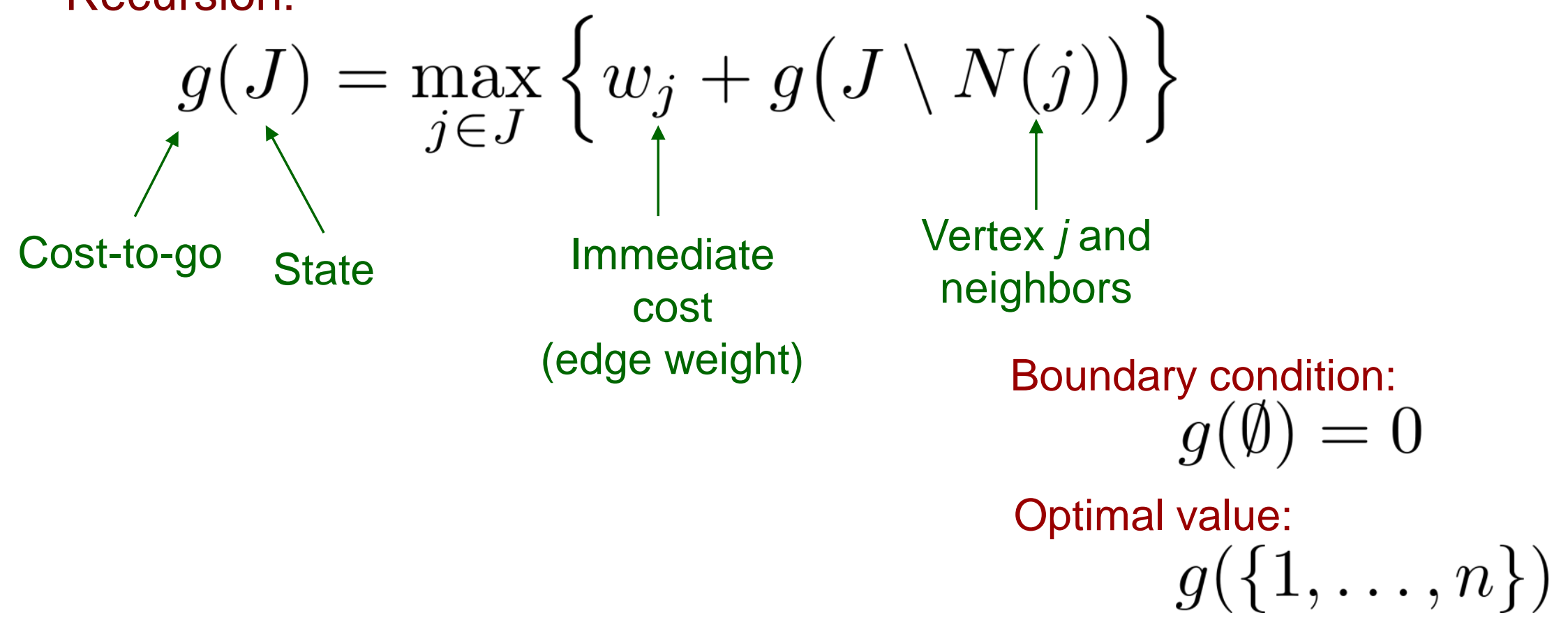




\section{Dynamic Programming Model}

- Max stable set problem on a graph.

- State $=$ set of vertices that can be added to stable set.

- State merger = union

Recursion:

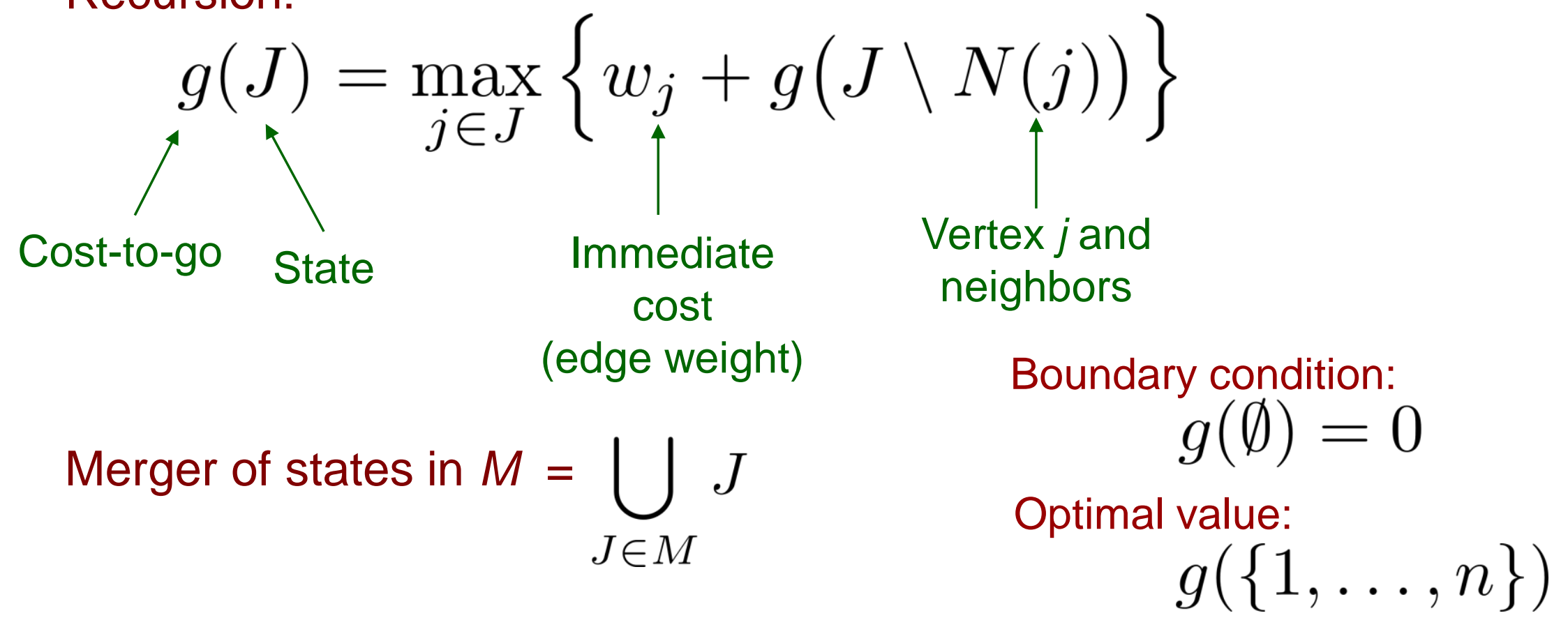




\section{Dynamic Programming Model}

- Single-machine scheduling with due dates

- Minimize total tardiness.

- State $=($ set of jobs not yet processed, latest finish time of jobs processed so far)

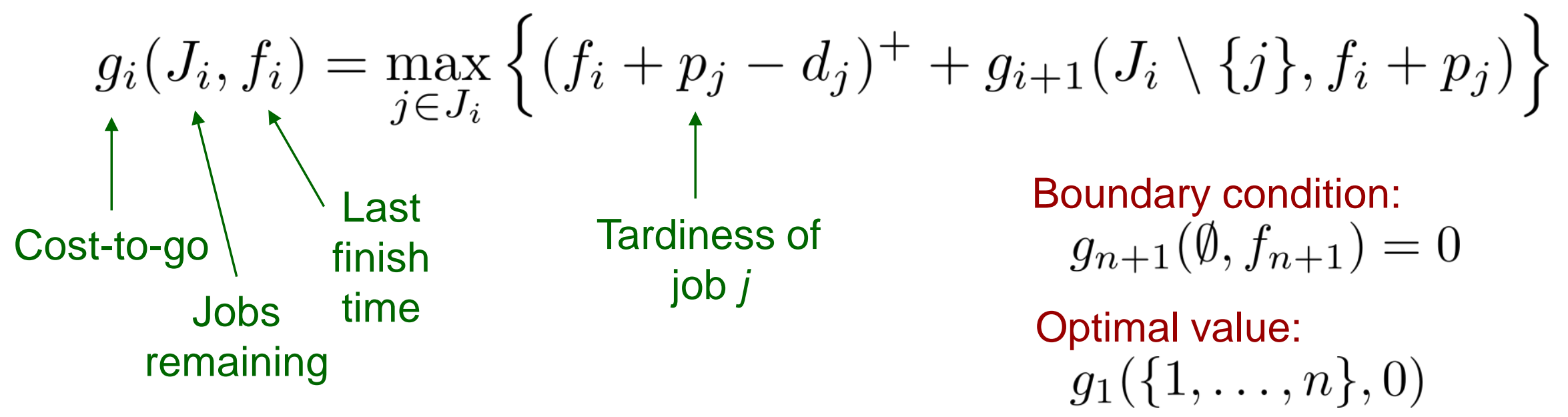




\section{Dynamic Programming Model}

- Single-machine scheduling with due dates

- Minimize total tardiness.

- State $=($ set of jobs not yet processed, latest finish time of jobs processed so far)

- State merger $=$ union, $\min$

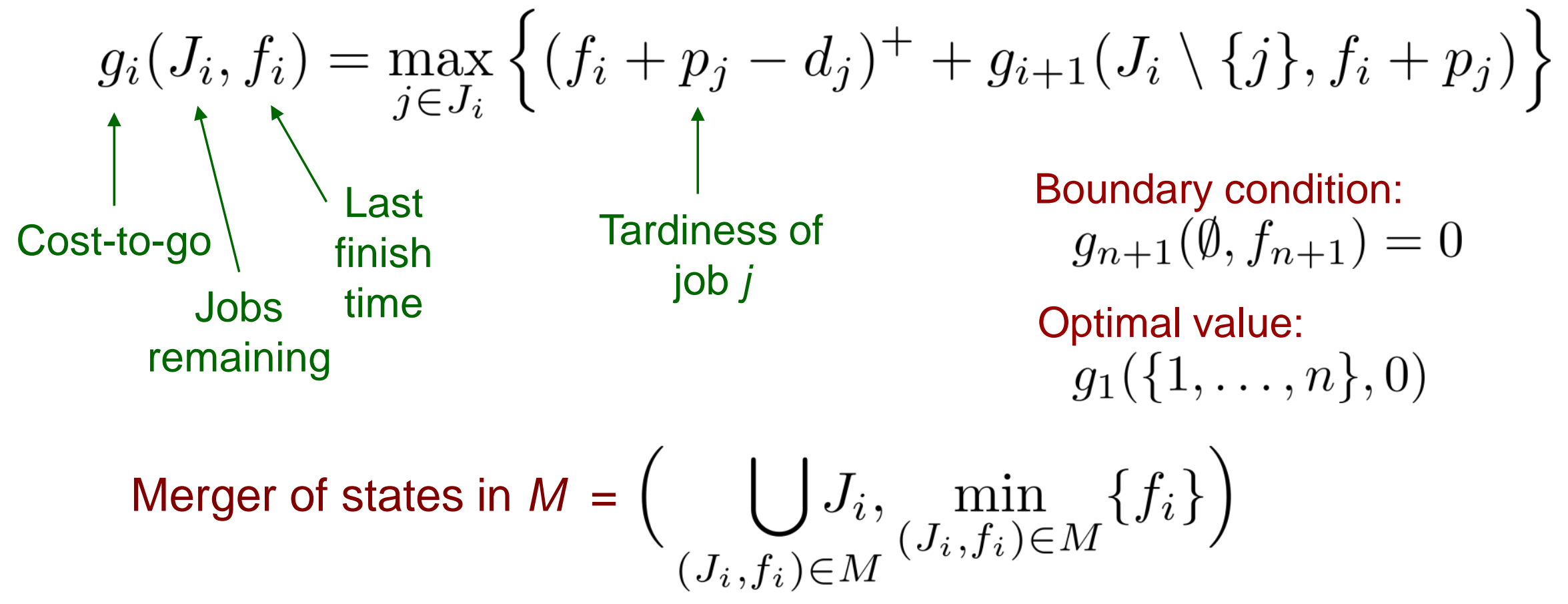




\section{Dynamic Programming Model}

- Single machine scheduling with due dates

- Easy to add constraints that are functions of current state

- Release times

- Shutdown periods

- Precedence constraints on jobs

- Easy to use more complicated cost function that is a function of current state

- Step functions, etc.

- Cost that depends on which jobs have been processed. 


\section{Dynamic Programming Model}

- Scheduling with sequence-dependent setup times

- State $=\left(J_{i}\right.$, last job processed, $\left.f_{i}\right)$

- State merger requires modification of states

$$
g_{i}\left(J_{i}, \ell_{i}, f_{i}\right)=\max _{j \in J_{i}}\left\{\left(f_{i}+p_{\ell_{i} j}-d_{j}\right)^{+}+g_{i+1}\left(J_{i} \backslash\{j\}, j, f_{i}+p_{\ell_{i} j}\right)\right\}
$$




\section{Dynamic Programming Model}

- Scheduling with sequence-dependent setup times

- To allow for state merger:

- State $=\left(J_{i}\right.$, set $L_{i}$ of pairs $\left(\ell_{i}, f_{i}\right)$, representing jobs

that could have been the last processed)

$\begin{aligned} g_{i}\left(J_{i}, L_{i}\right)=\max _{j \in J_{i}}\{ & \left(\min _{\left(\ell_{i}, f_{i}\right) \in L_{i}}\left\{f_{i}+p_{\ell_{i} j}\right\}-d_{j}\right)^{+} \\ & \left.+g_{i+1}\left(J_{i} \backslash\{j\},\left\{\left(j, \min _{\left(\ell_{i}, f_{i}\right) \in L_{i}}\left\{f_{i}+p_{\ell_{i} j}\right\}\right)\right\}\right)\right\}\end{aligned}$

Merger of states in $M=\left(\bigcup J_{i}, \bigcup L_{i},\right)$ 


\section{Dynamic Programming Model}

- Max cut problem on a graph.

- Partition nodes into 2 sets so as to maximize total weight of connecting edges.

- State = marginal benefit of placing each remaining vertex on left side of cut.

- State merger $=$

- Componentwise min if all components $\geq 0$ or all $\leq 0$; 0 otherwise

- Adjust incoming arc weights

- Max 2-SAT.

- Similar to max cut. 


\section{Branching Algorithm}

- Solve optimization problem using a novel branch-and-bound algorithm.

- Branch on nodes in last exact layer of relaxed decision diagram.

- ...rather than branch on variables.

- Create a new relaxed DD rooted at each branching node.

- Prune search tree using bounds from relaxed DD. 


\section{Branching Algorithm}

- Solve optimization problem using a novel branch-and-bound algorithm.

- Branch on nodes in last exact layer of relaxed decision diagram.

- ...rather than branch on variables.

- Create a new relaxed DD rooted at each branching node.

- Prune search tree using bounds from relaxed DD.

- Advantage: a manageable number states may be reachable in first few layers.

- ...even if the state space is exponential.

- Alternative way of dealing with curse of dimensionality. 


\section{Branching Algorithm}

Branching in a relaxed decision diagram

Diagram is exact down to here

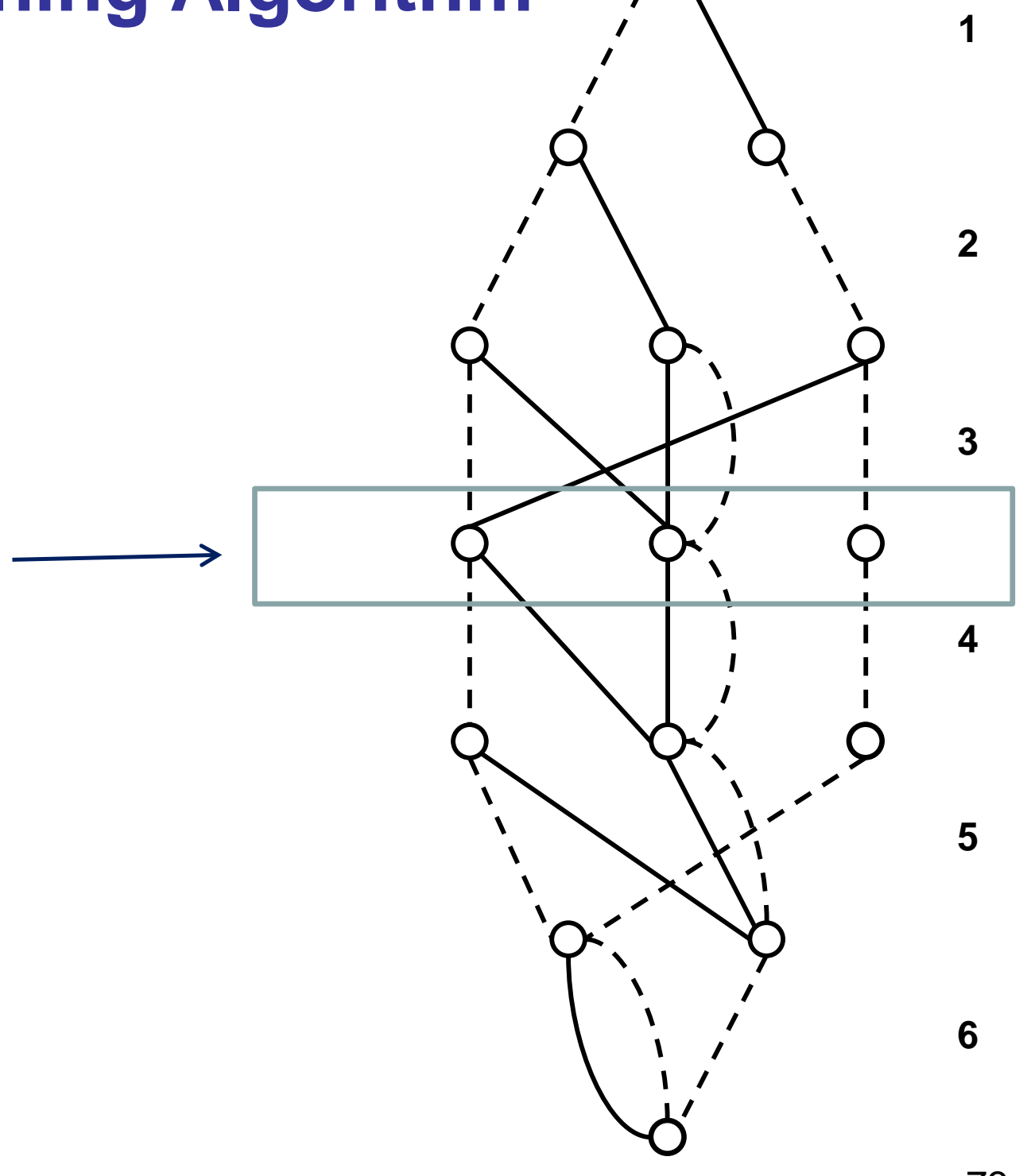




\section{Branching Algorithm}

Branching in a relaxed decision diagram

Branch on nodes in this layer

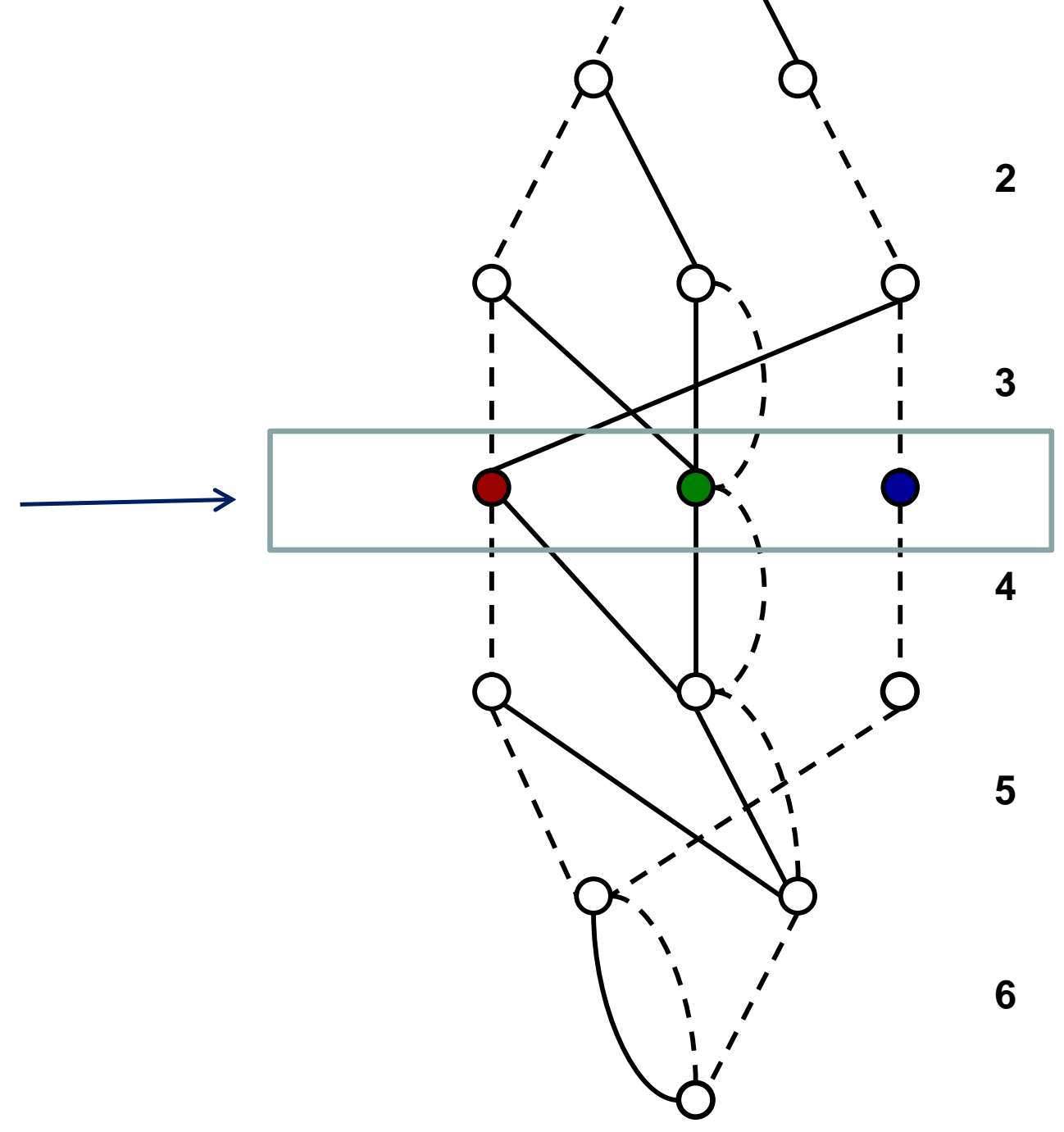




\section{Branching Algorithm}

Branching in a relaxed

decision diagram

3

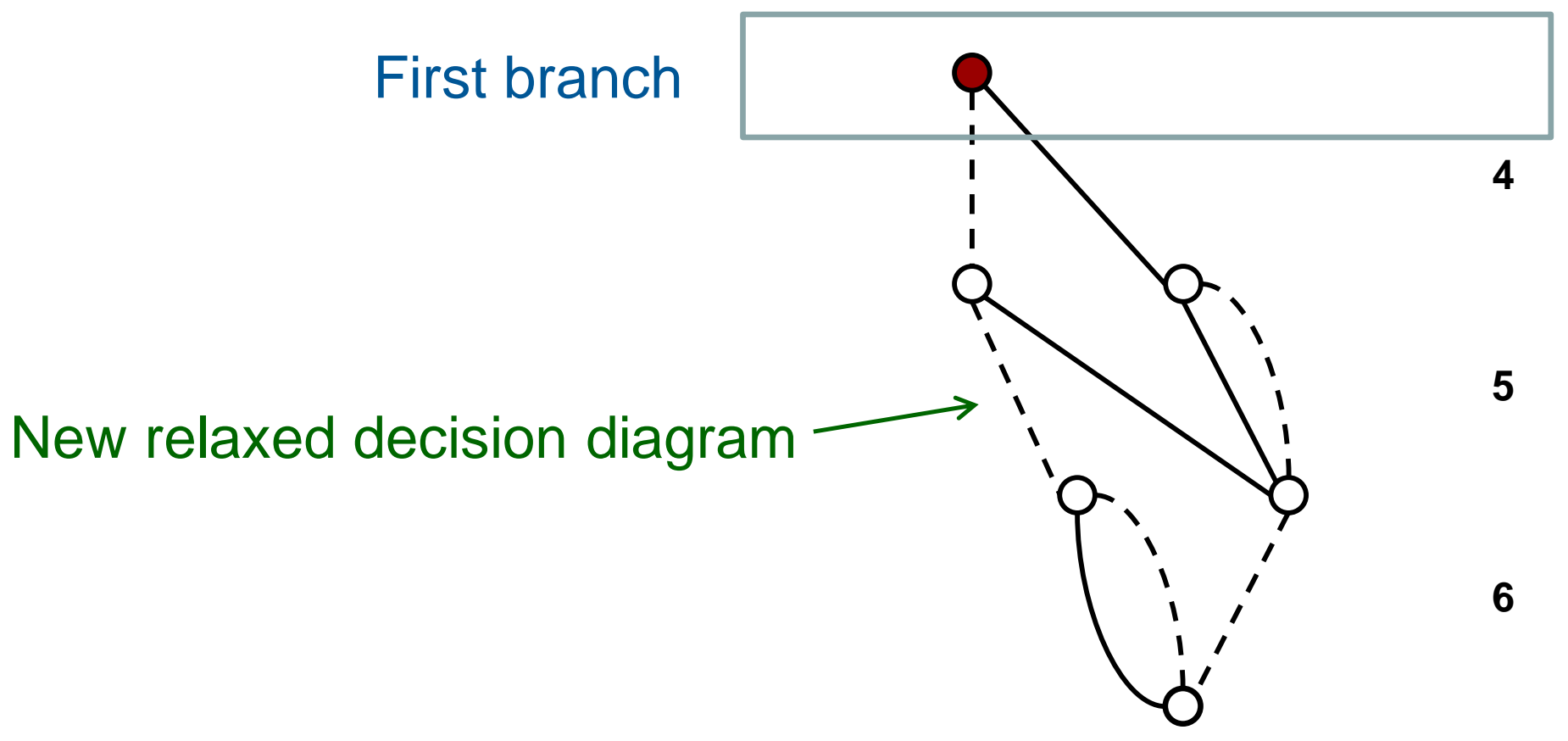




\section{Branching Algorithm}

Branching in a relaxed

decision diagram

3

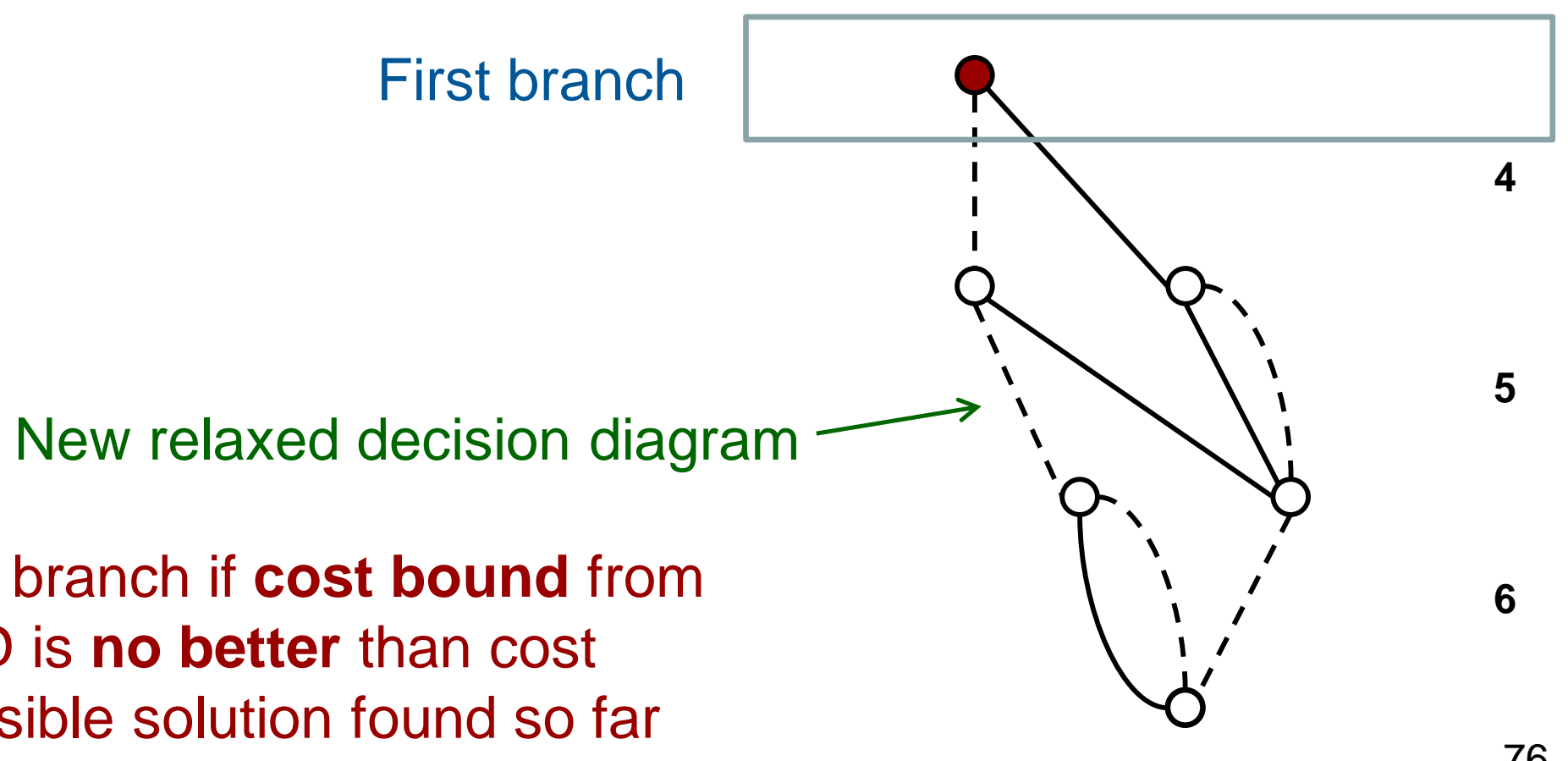

Prune this branch if cost bound from relaxed DD is no better than cost of best feasible solution found so far (branch and bound). 


\section{Branching Algorithm}

Branching in a relaxed

decision diagram

3

Second branch

Prune this branch if cost bound from relaxed DD is no better than cost of best feasible solution found so far (branch and bound).

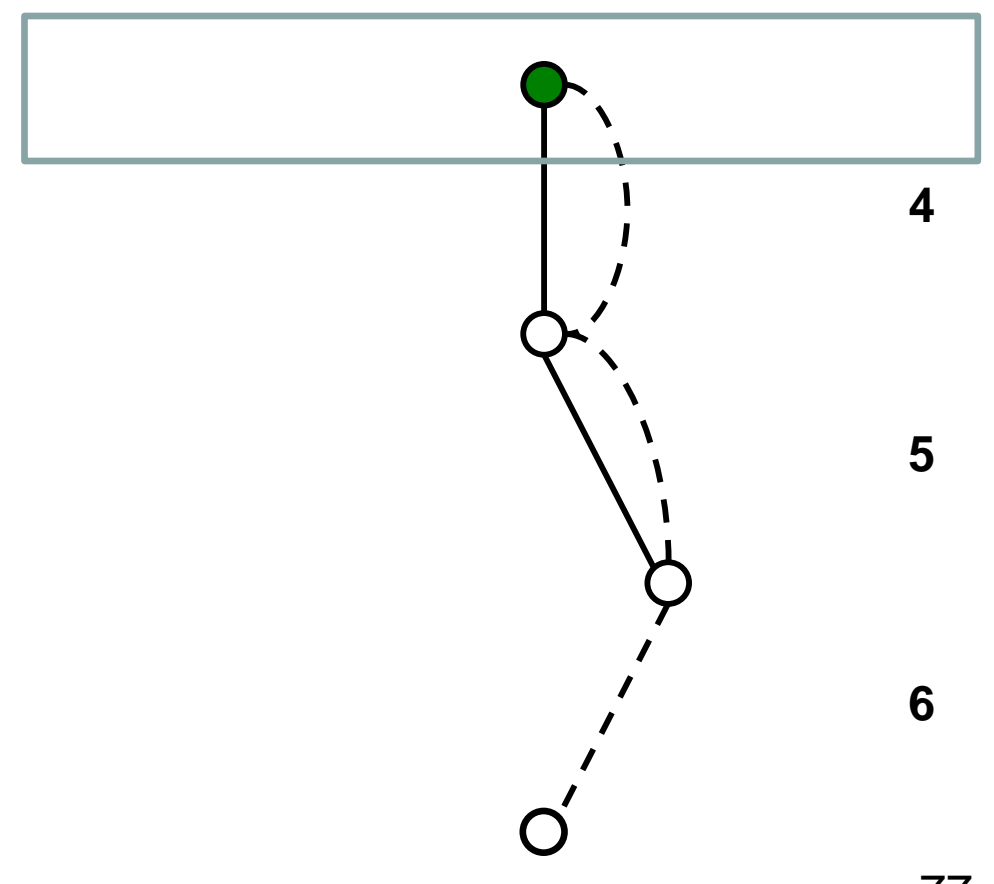




\section{Branching Algorithm}

Branching in a relaxed decision diagram

3

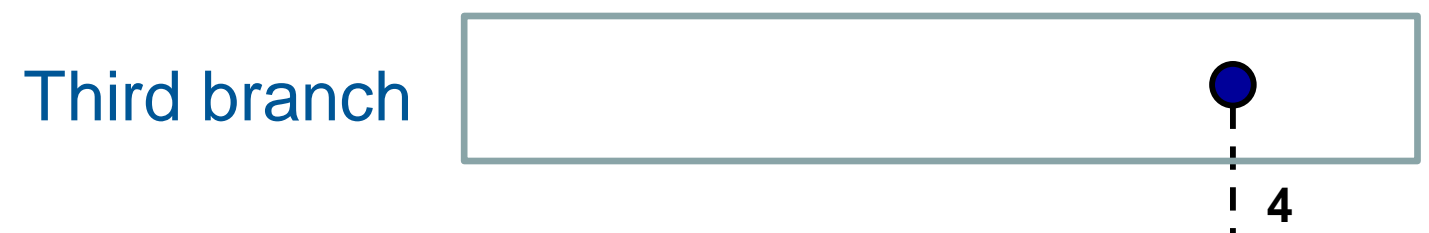

Prune this branch if cost bound from relaxed DD is no better than cost of best feasible solution found so far (branch and bound).

Continue recursively 


\section{State Space Relaxation?}

- This is very different from state space relaxation.

- Problem is not solved by dynamic programming.

- Relaxation created by merging nodes of DD

- ...rather than mapping into smaller state space.

- Relaxation is constructed dynamically

- ....as relaxed DD is built.

- Relaxation uses same state variables as exact formulation

- ...which allows branching in relaxed DD

Christofides, Mingozzi, Toth (1981) 


\section{Computational performance}

- Computational results...

- Applied to stable set, max cut, max 2-SAT.

- Superior to commercial MIP solver (CPLEX) on most instances.

- Obtained best known solution on some max cut instances.

- Slightly slower than MIP on stable set with precomputed clique cover model, but...

Bergman, Ciré, van Hoeve, JH (2016) 


\section{Computational performance}

Max cut on a graph

Avg. solution time vs

graph density

30 vertices

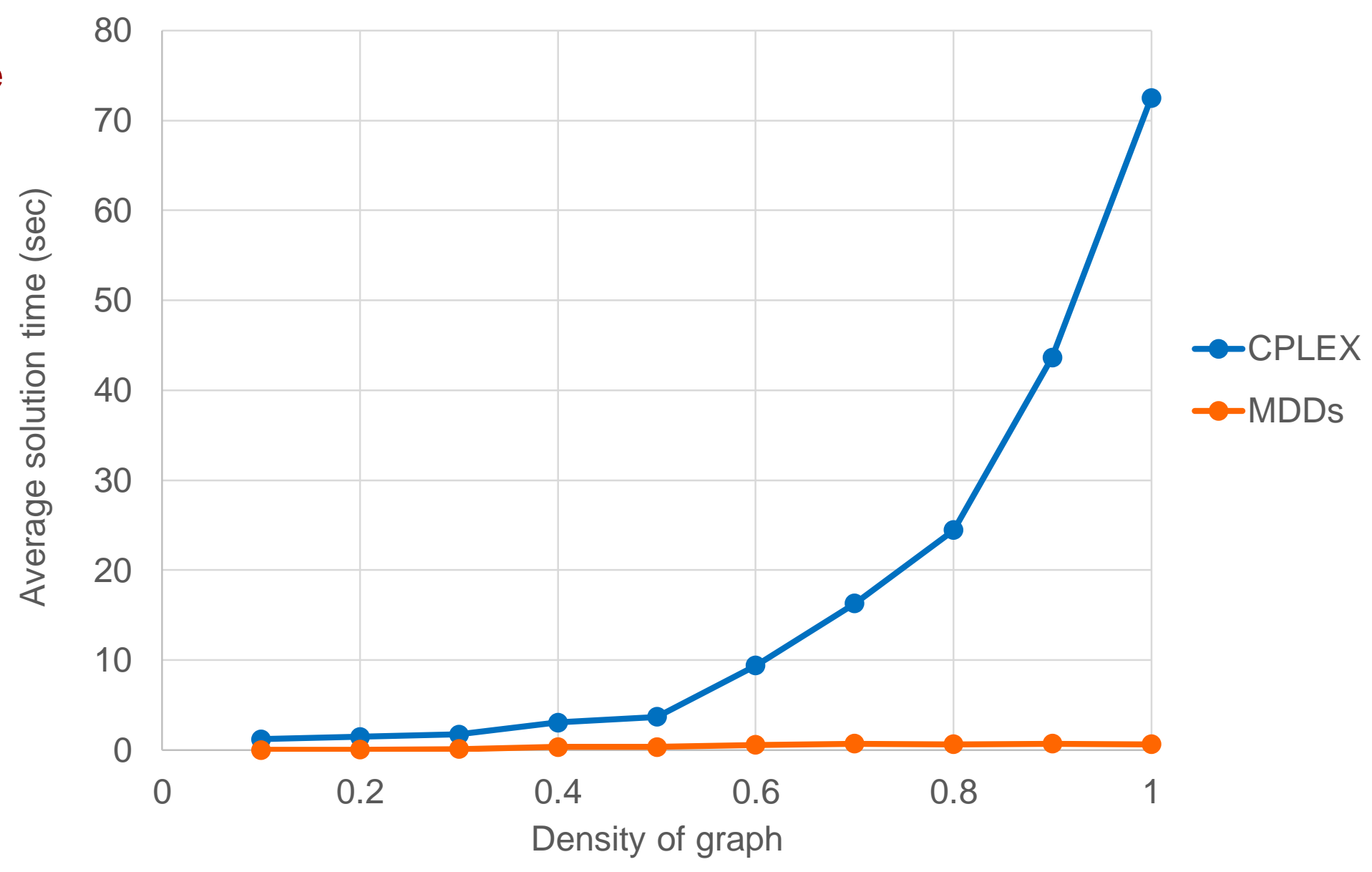




\section{Computational performance}

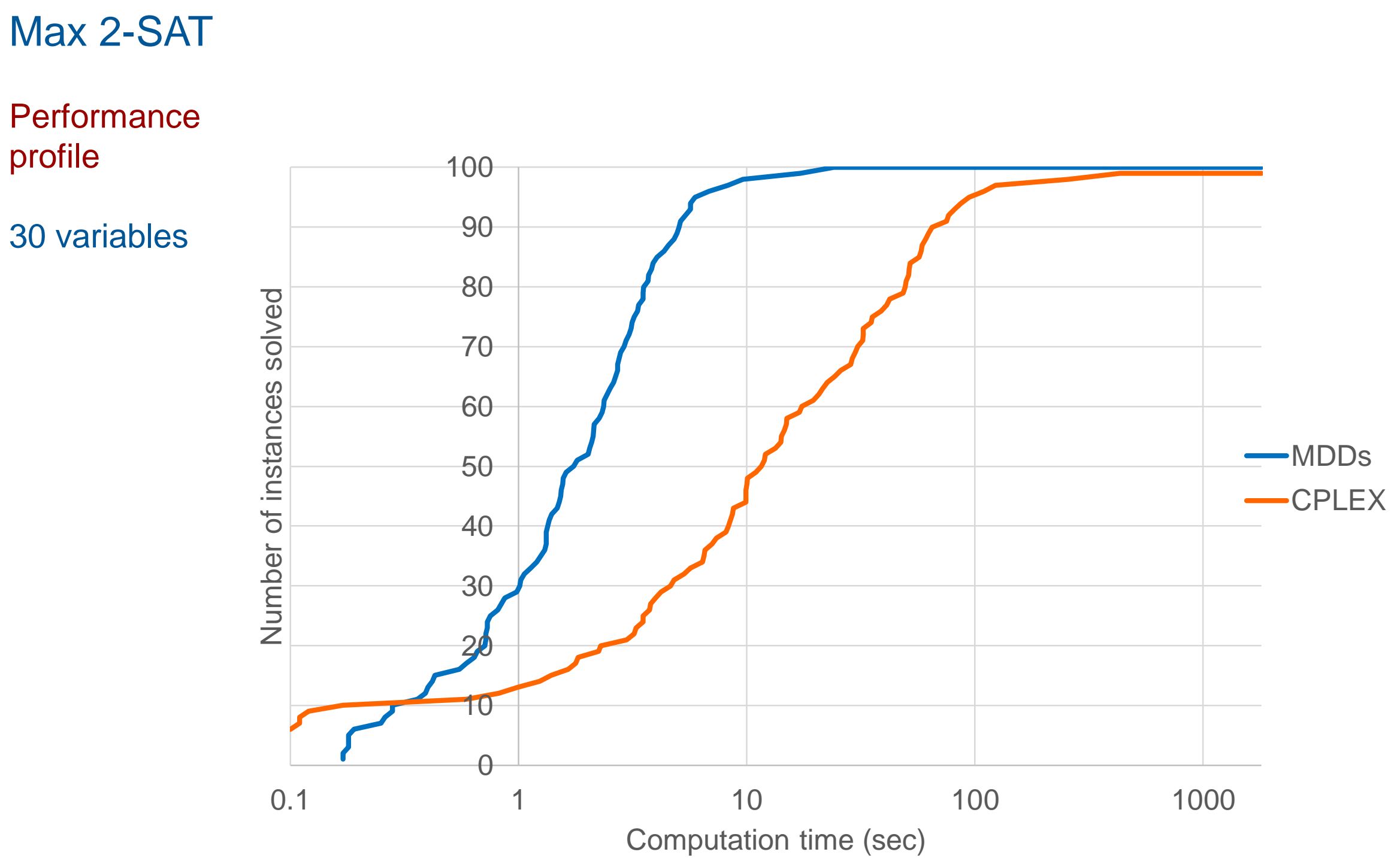




\section{Computational performance}

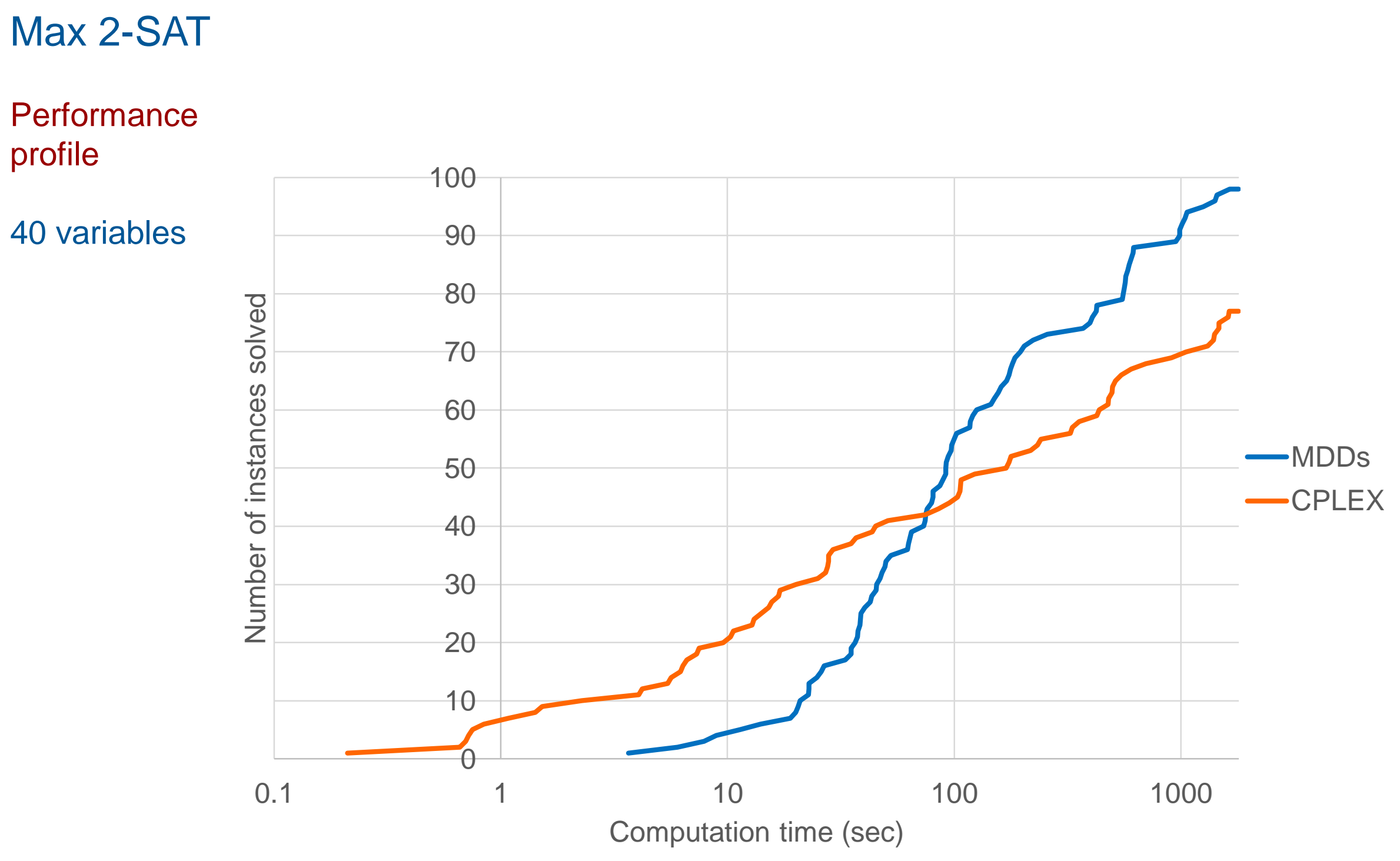




\section{Computational performance}

- Potential to scale up

- No need to load large inequality model into solver.

- Parallelizes very effectively

- Near-linear speedup.

- Much better than mixed integer programming. 


\section{Computational performance}

- In all computational comparisons so far...

- Problem is easily formulated for IP.

- DD-based optimization is most competitive when...

- Problem has a recursive dynamic programming model...

- and no convenient IP model. 


\section{Computational performance}

- In all computational comparisons so far...

- Problem is easily formulated for IP.

- DD-based optimization is most competitive when...

- Problem has a recursive dynamic programming model...

- and no convenient IP model.

- Such as...

- Sequencing and scheduling problems (next talk)

- Planning problems

- DP problems with exponential state space

- New approach to "curse of dimensionality"

- Problems with nonconvex, nonseparable objective function... 


\section{Modeling the Objective Function}

- Weighted DD can represent any objective function

- Separable functions are the easiest, but any nonseparable function is possible.

- Can be nonlinear, nonconvex, etc.

- The issue is complexity of resulting DD 


\section{Modeling the Objective Function}

- Weighted DD can represent any objective function

- Separable functions are the easiest, but any nonseparable function is possible.

- Can be nonlinear, nonconvex, etc.

- The issue is complexity of resulting DD

- Multiple encodings

- A given objective function can be encoded by multiple assignments of costs to arcs.

- There is a unique canonical arc cost assignment.

- Which can reduce size of exact DD.

- Design state variables accordingly 


\section{Modeling the Objective Function}

Set covering with separable cost function

Easy. Just label arcs with weights.

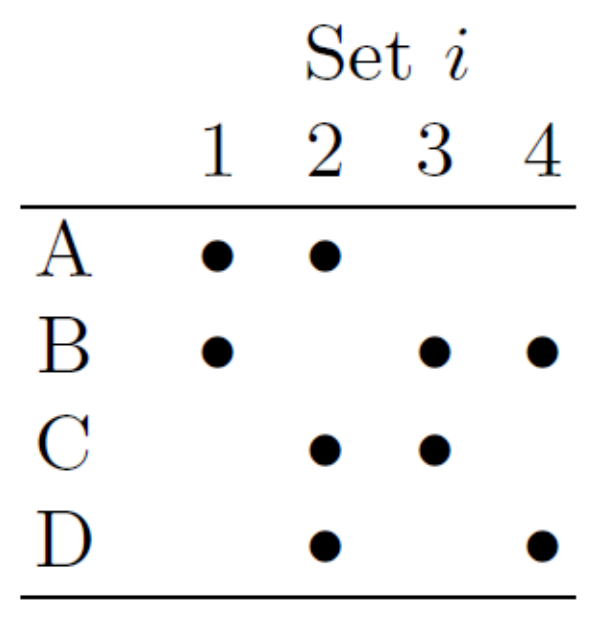

Weight $\quad 3 \quad 5 \quad 4 \quad 6$

$x_{i}=1$ when we select set $i$

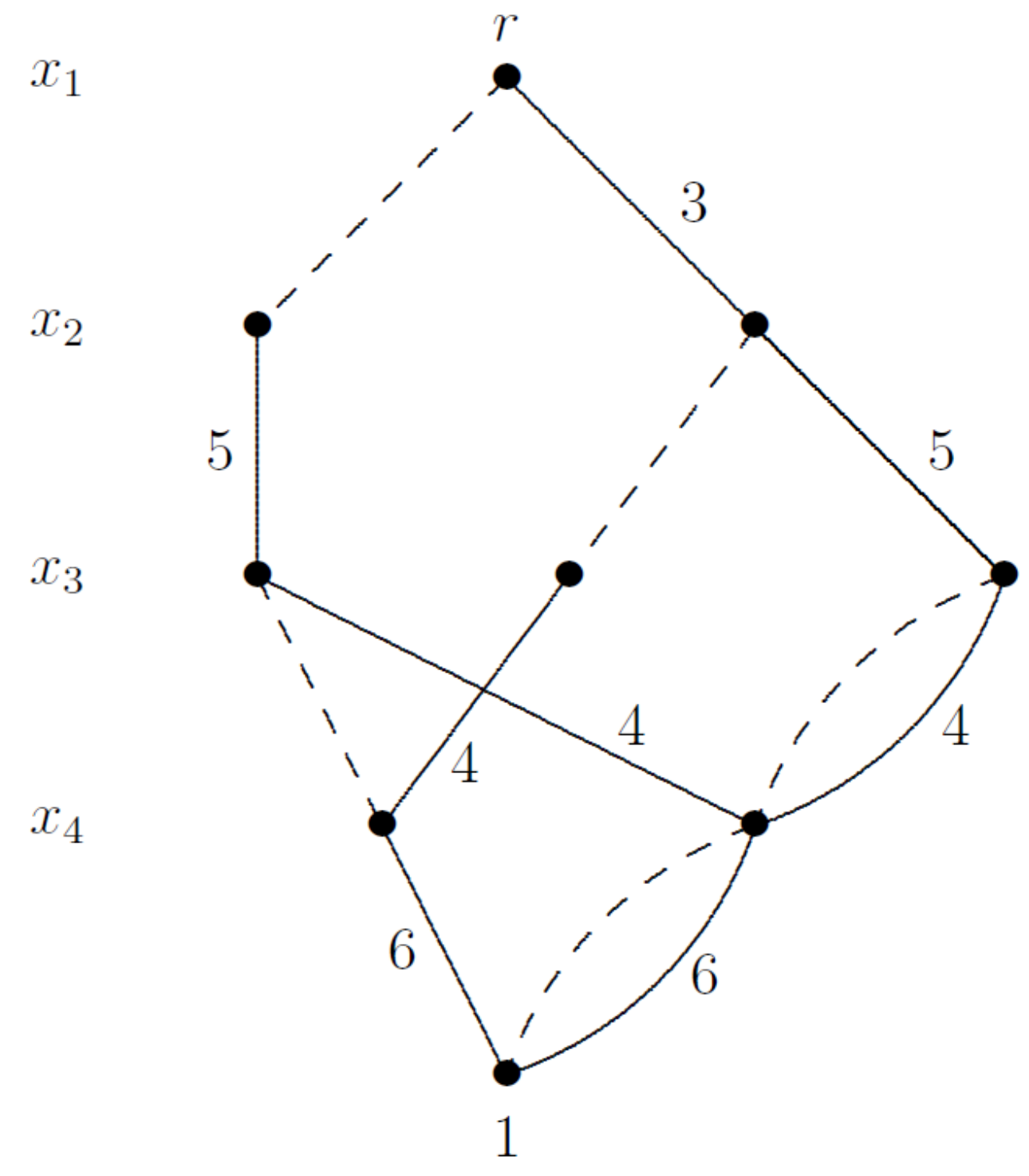




\section{Modeling the Objective Function}

Nonseparable cost function

Now what?

\begin{tabular}{cc}
$x$ & $f(x)$ \\
\hline$(0,1,0,1)$ & 6 \\
$(0,1,1,0)$ & 7 \\
$(0,1,1,1)$ & 8 \\
$(1,0,1,1)$ & 5 \\
$(1,1,0,0)$ & 6 \\
$(1,1,0,1)$ & 8 \\
$(1,1,1,0)$ & 7 \\
$(1,1,1,1)$ & 9 \\
\hline
\end{tabular}

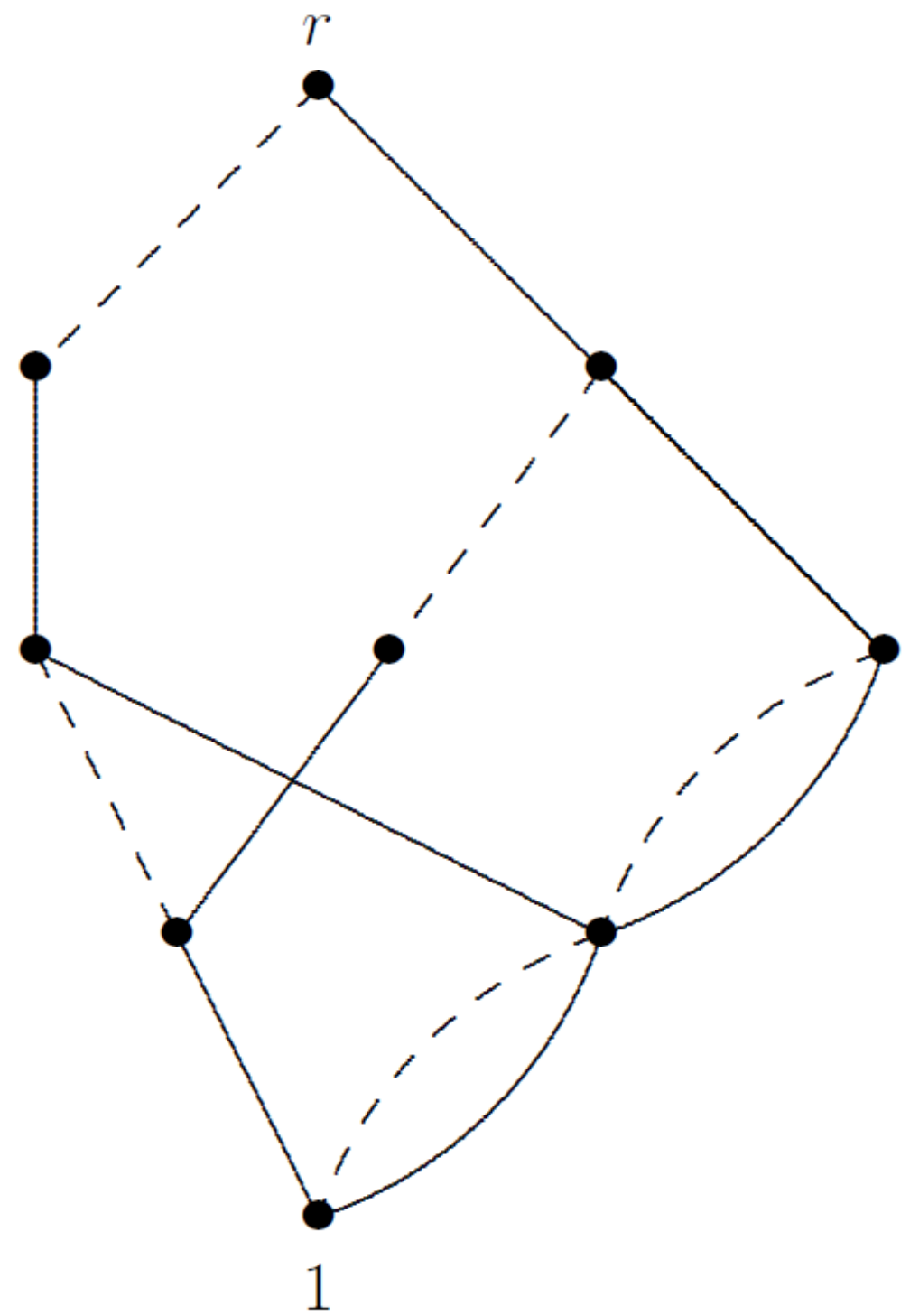




\section{Modeling the Objective Function}

Nonseparable cost function

Put costs on leaves of branching tree.

\begin{tabular}{cc}
$x$ & $f(x)$ \\
\hline$(0,1,0,1)$ & 6 \\
$(0,1,1,0)$ & 7 \\
$(0,1,1,1)$ & 8 \\
$(1,0,1,1)$ & 5 \\
$(1,1,0,0)$ & 6 \\
$(1,1,0,1)$ & 8 \\
$(1,1,1,0)$ & 7 \\
$(1,1,1,1)$ & 9 \\
\hline
\end{tabular}
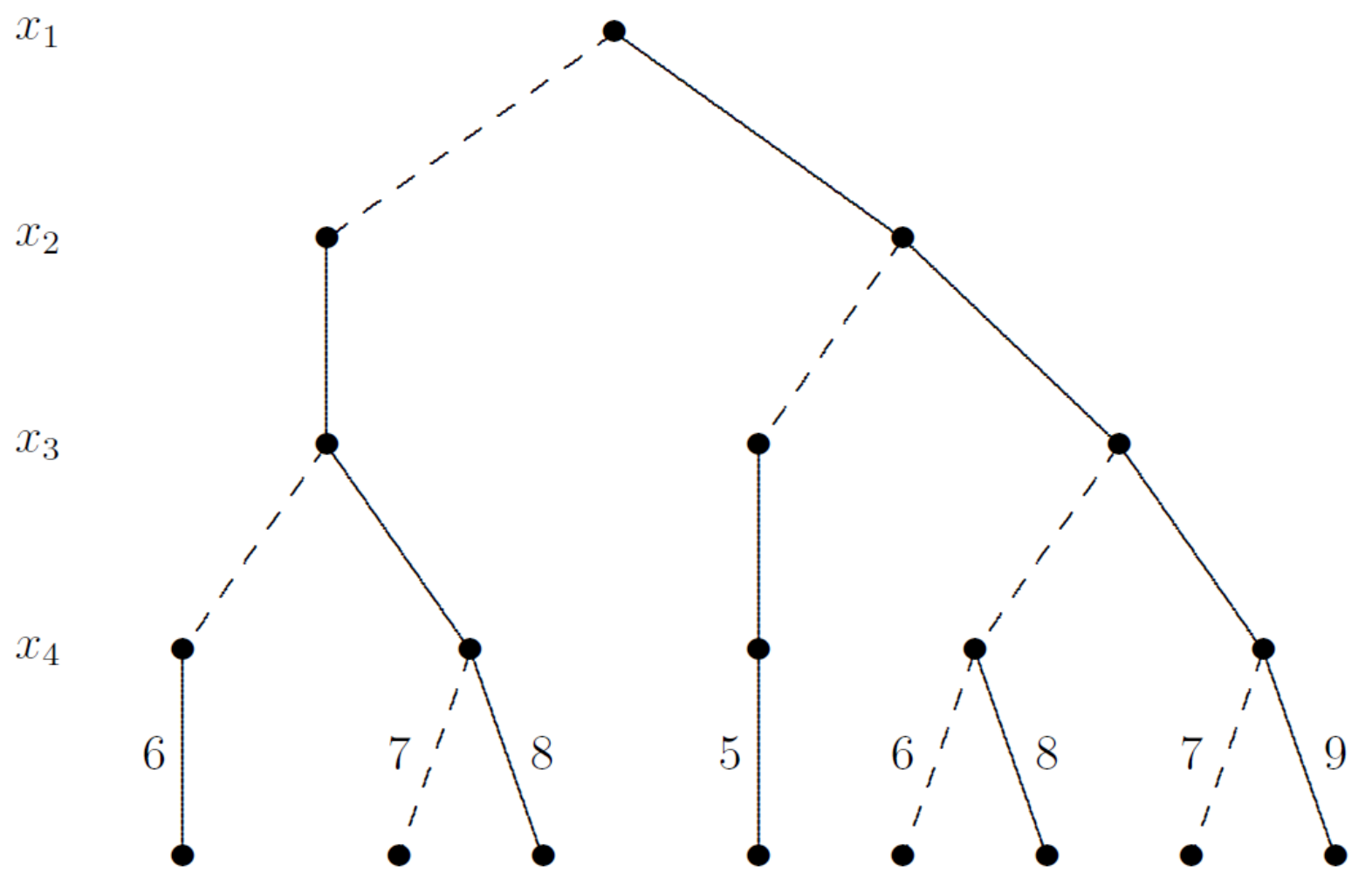


\section{Modeling the Objective Function}

Nonseparable cost function

Put costs on leaves of branching tree.

But now we can't reduce the tree to an efficient decision diagram.

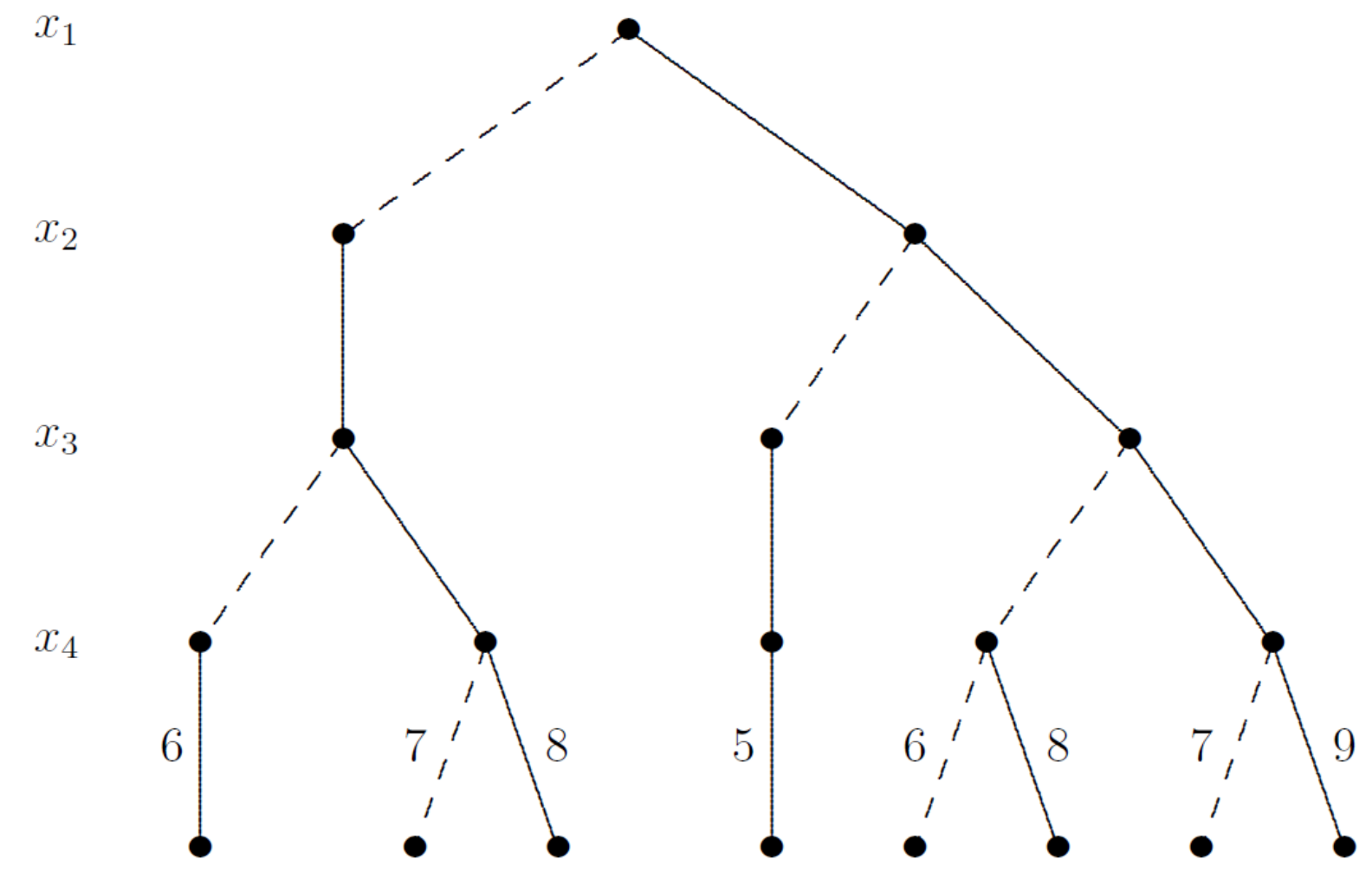




\section{Modeling the Objective Function}

\section{Nonseparable cost function}

Put costs on leaves of branching tree.

But now we can't reduce the tree to an efficient decision diagram.

We will rearrange costs to obtain canonical costs.

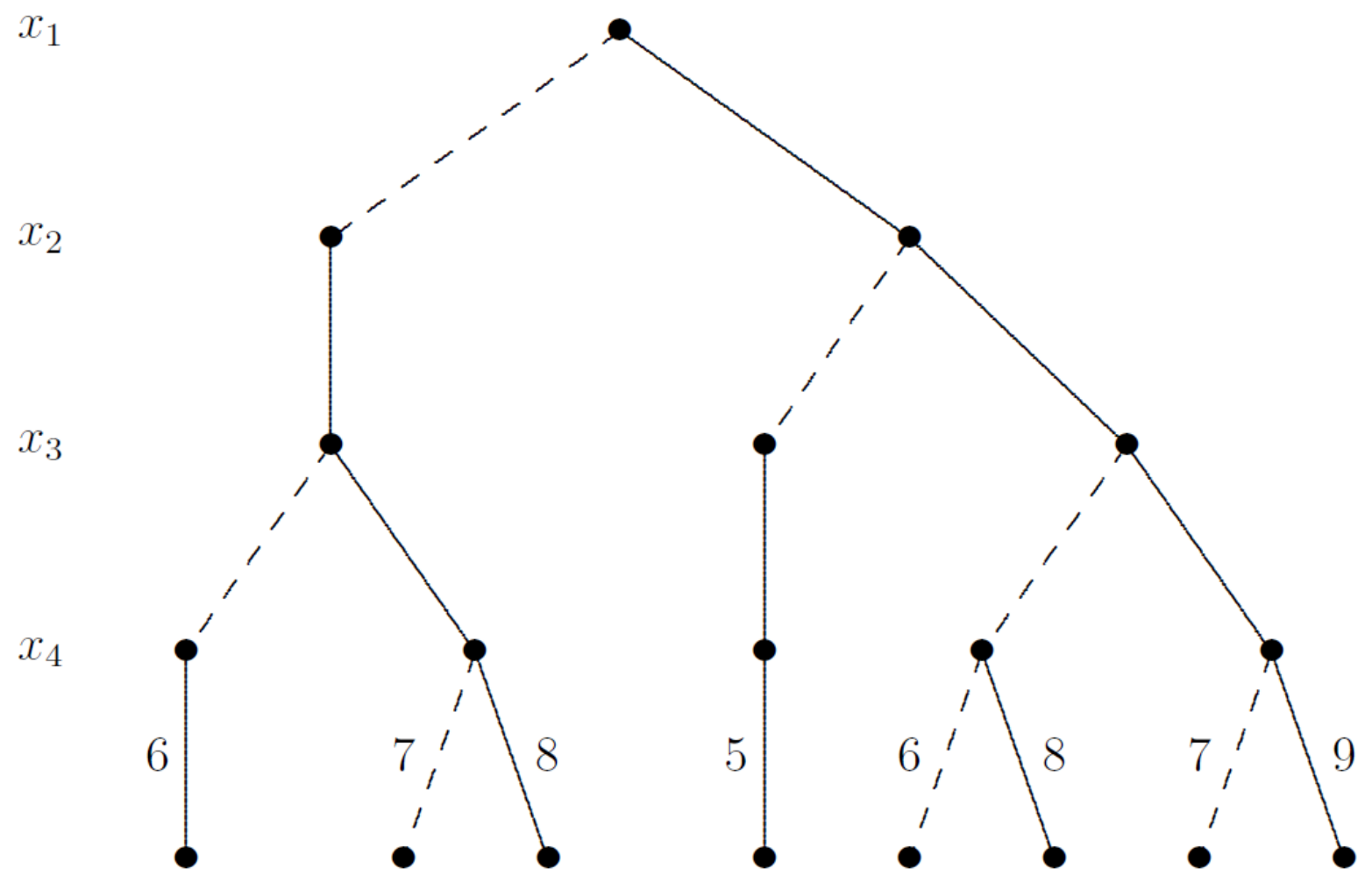




\section{Modeling the Objective Function}

\section{Nonseparable cost function}

Put costs on leaves of branching tree.

But now we can't reduce the tree to an efficient decision diagram.

We will rearrange costs to obtain canonical costs.

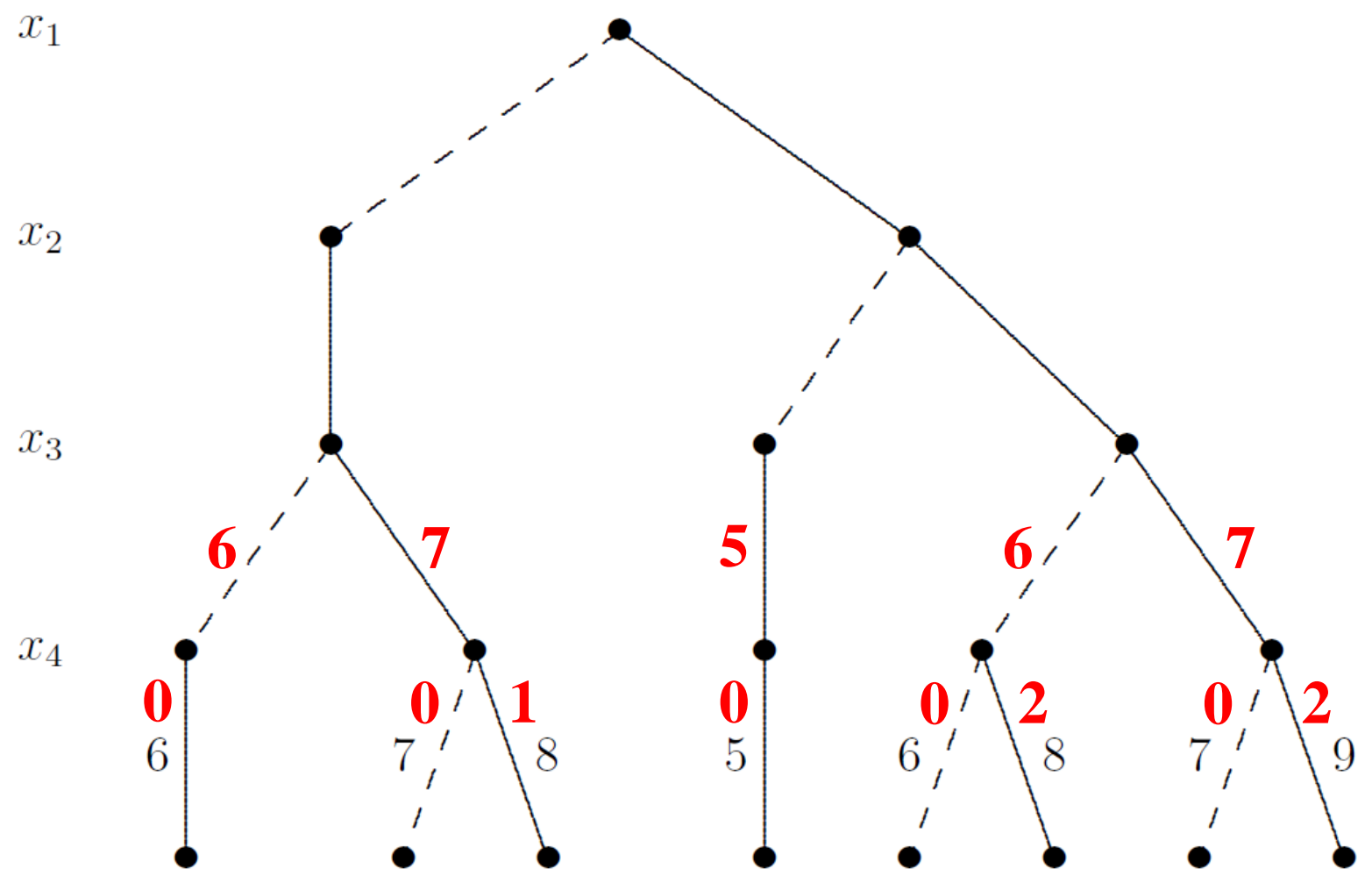




\section{Modeling the Objective Function}

\section{Nonseparable cost function}

Put costs on leaves of branching tree.

But now we can't reduce the tree to an efficient decision diagram.

We will rearrange costs to obtain canonical costs.

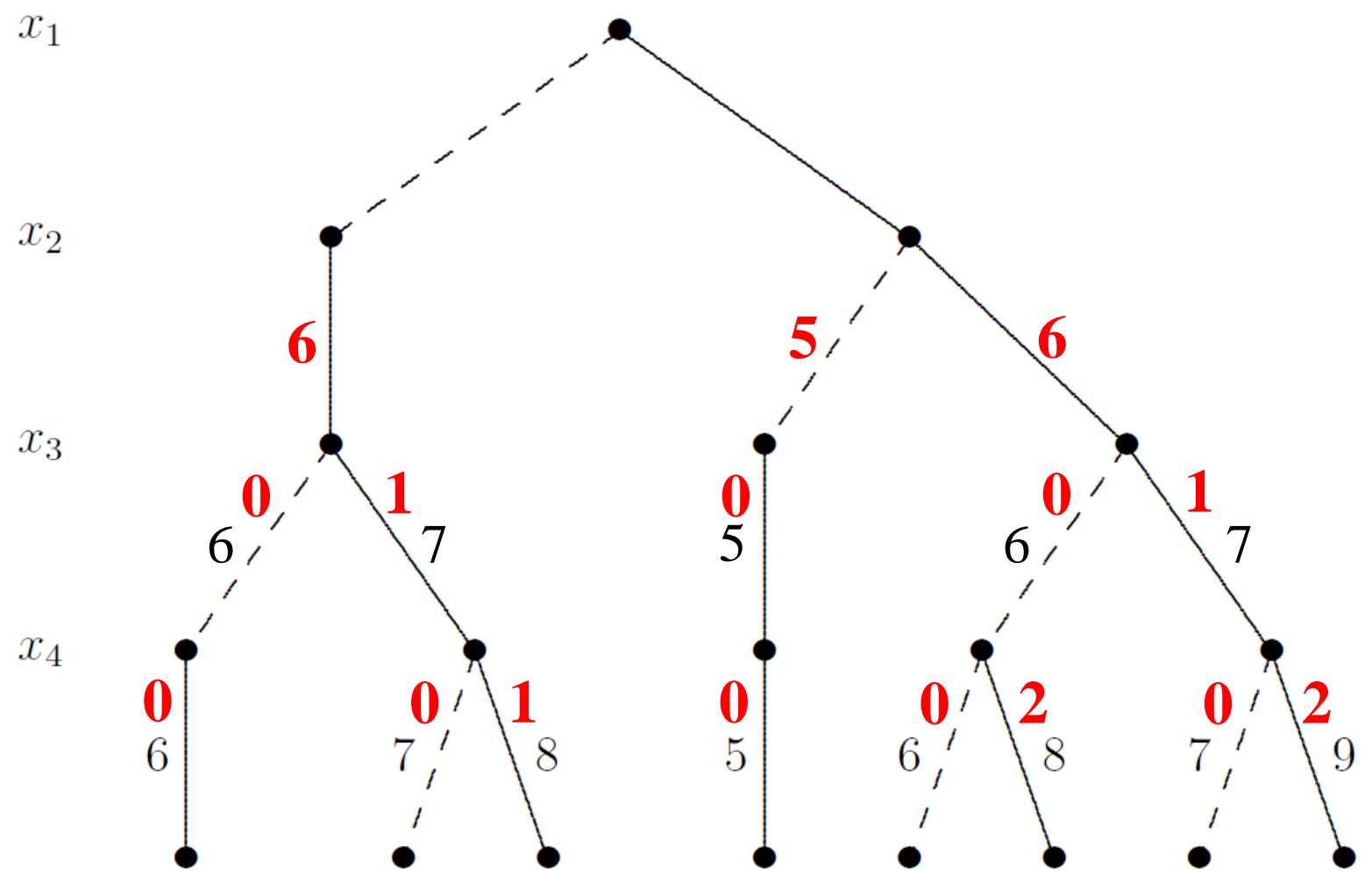




\section{Modeling the Objective Function}

\section{Nonseparable cost function}

Put costs on leaves of branching tree.

But now we can't reduce the tree to an efficient decision diagram.

We will rearrange costs to obtain canonical costs.

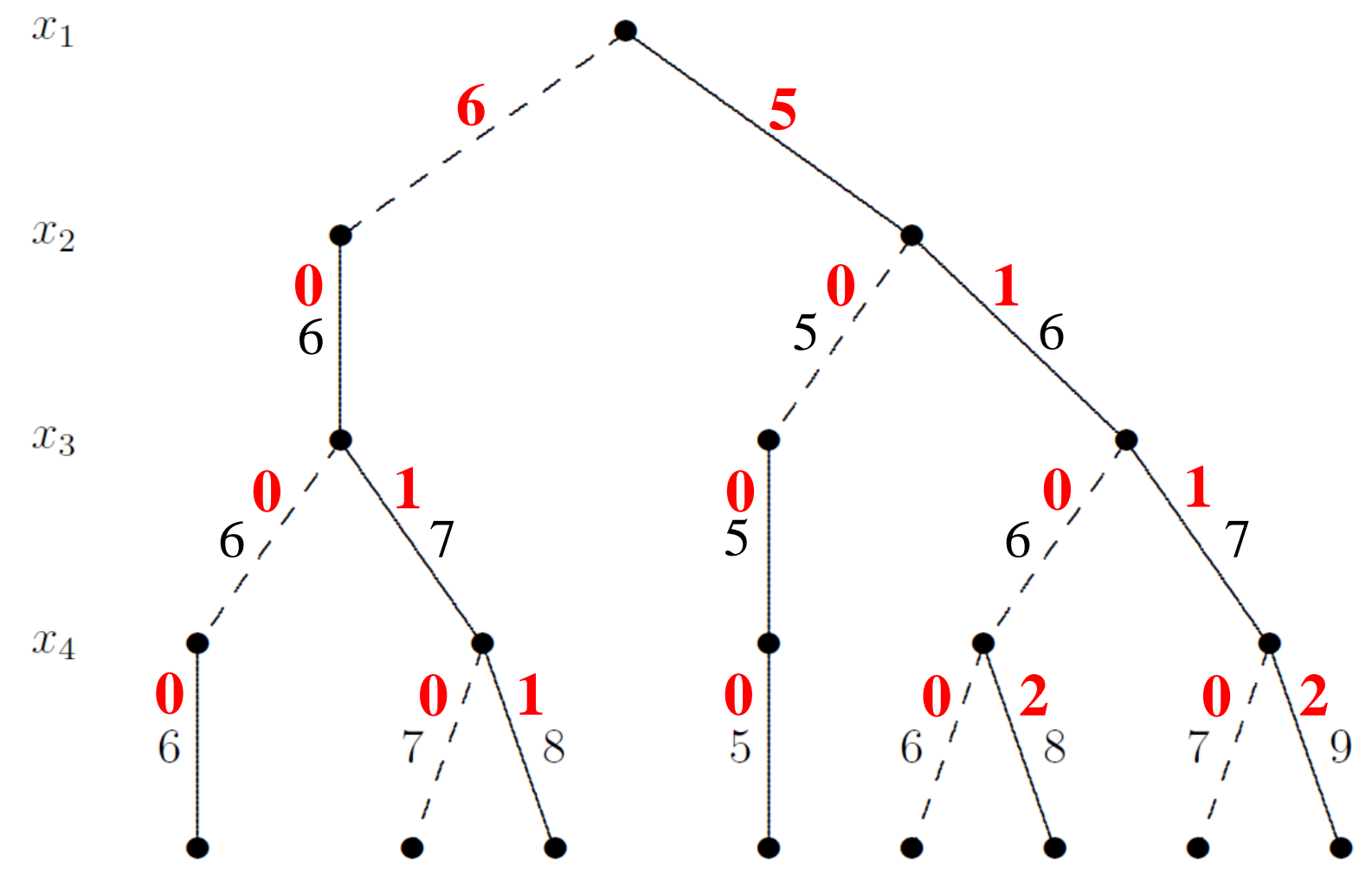




\section{Modeling the Objective Function}

Nonseparable cost function

Now the tree can be reduced.

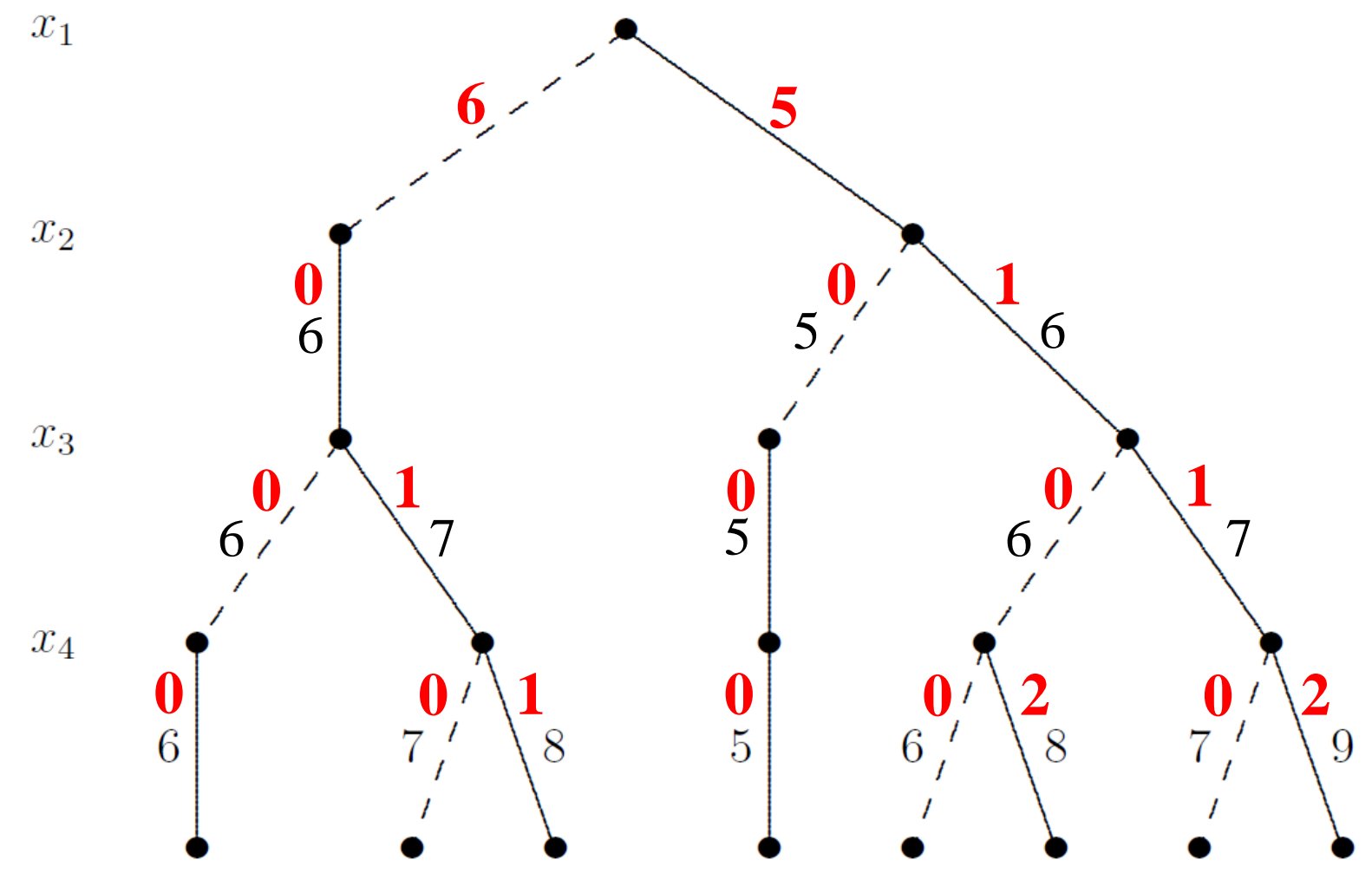




\section{Modeling the Objective Function}

Nonseparable cost function

Now the tree can be reduced.

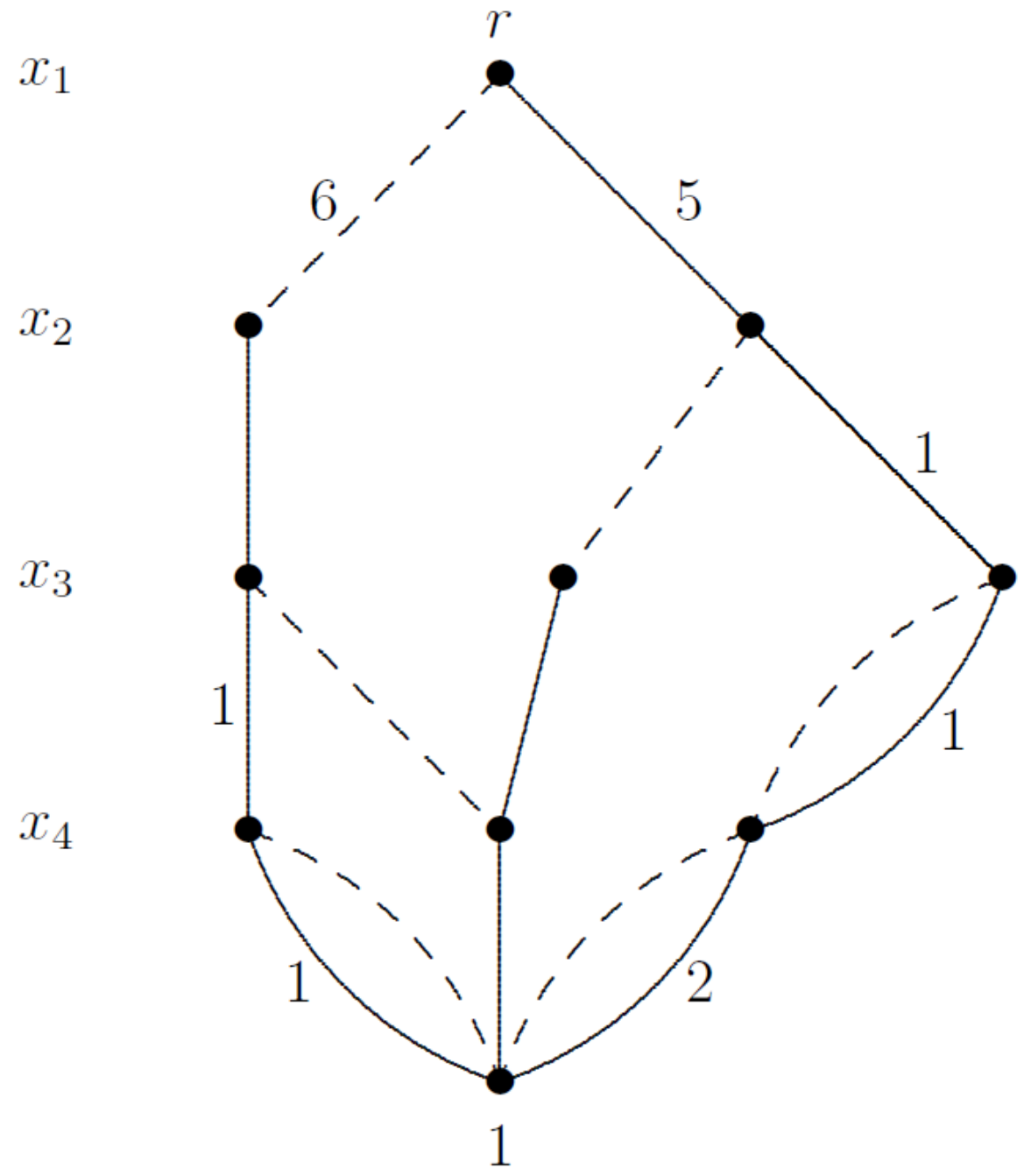




\section{Modeling the Objective Function}

\section{Nonseparable cost function}

DD is larger than

reduced unweighted DD, but still compact.

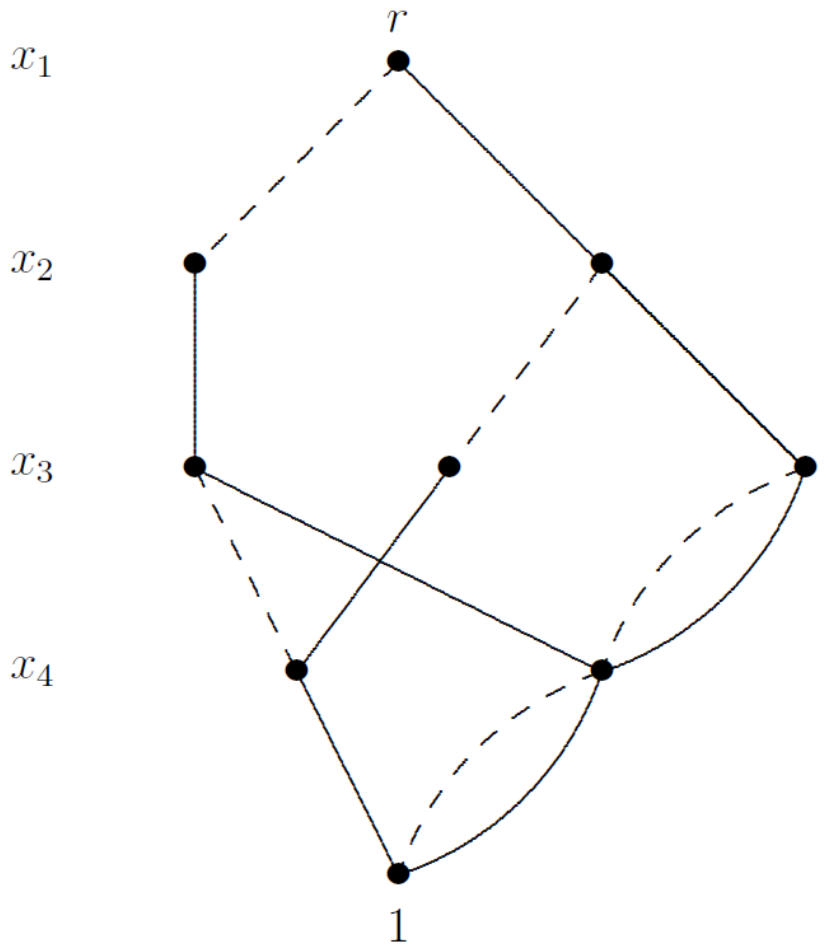

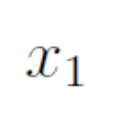

$x_{2}$

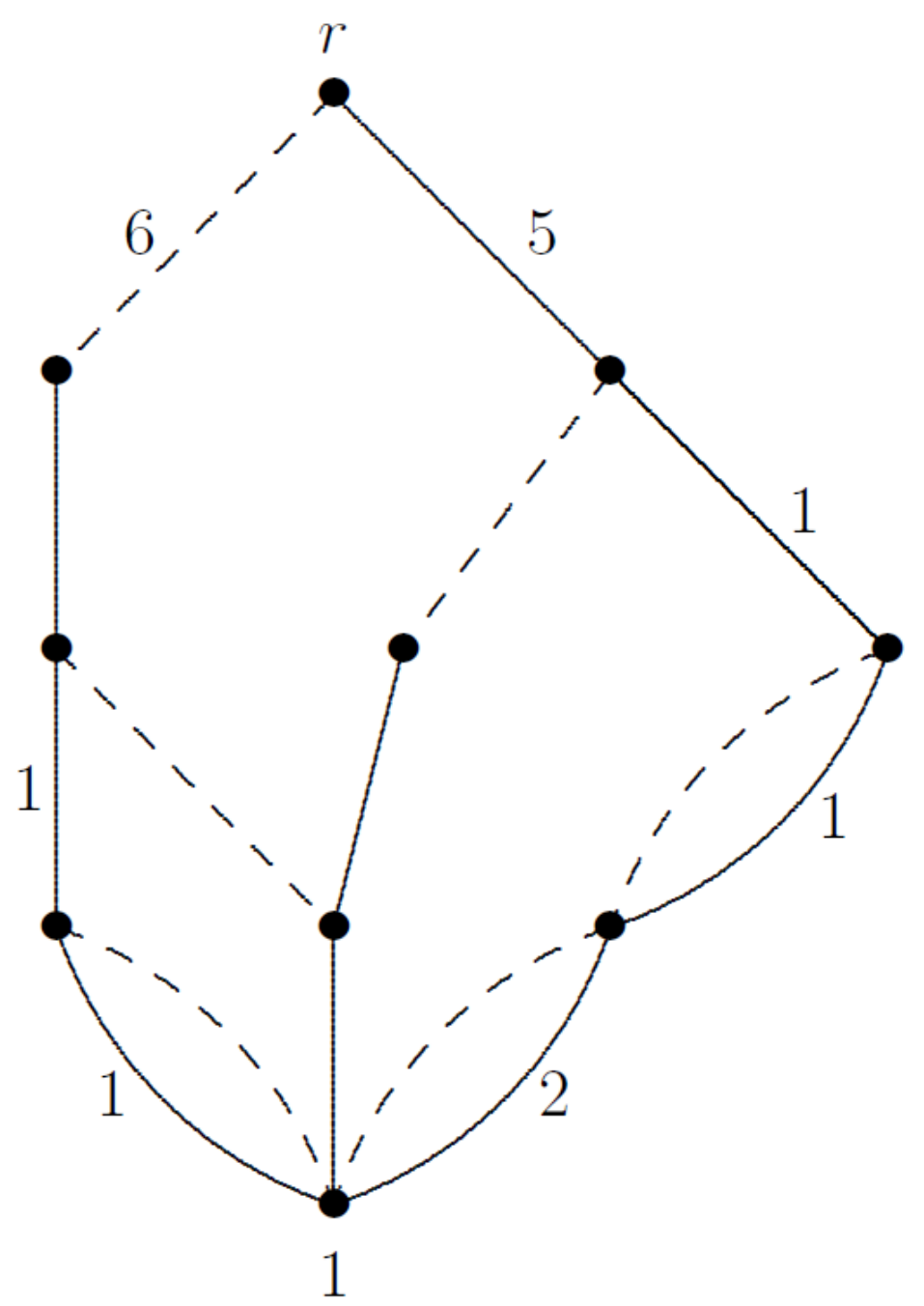




\section{Modeling the Objective Function}

Theorem. For a given variable ordering, a given objective function is represented by a unique weighted decision diagram with canonical costs.

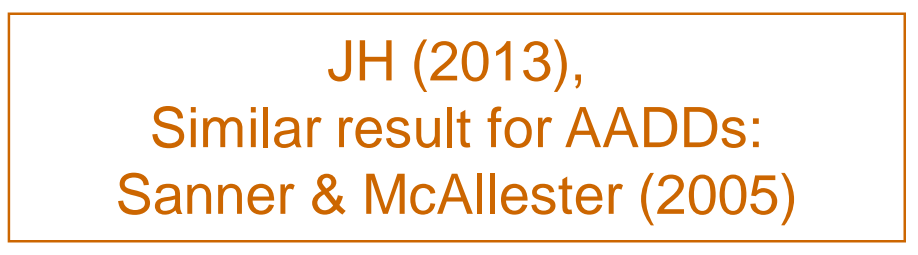




\section{Inventory Management Example}

- In each period $i$, we have:

- Demand $d_{i}$

- Unit production cost $c_{i}$

- Warehouse space $m$

- Unit holding cost $h_{i}$

- In each period, we decide:

- Production level $x_{i}$

- Stock level $s_{i}$

- Objective:

- Meet demand each period while minimizing production and holding costs. 


\section{Reducing the Transition Graph}

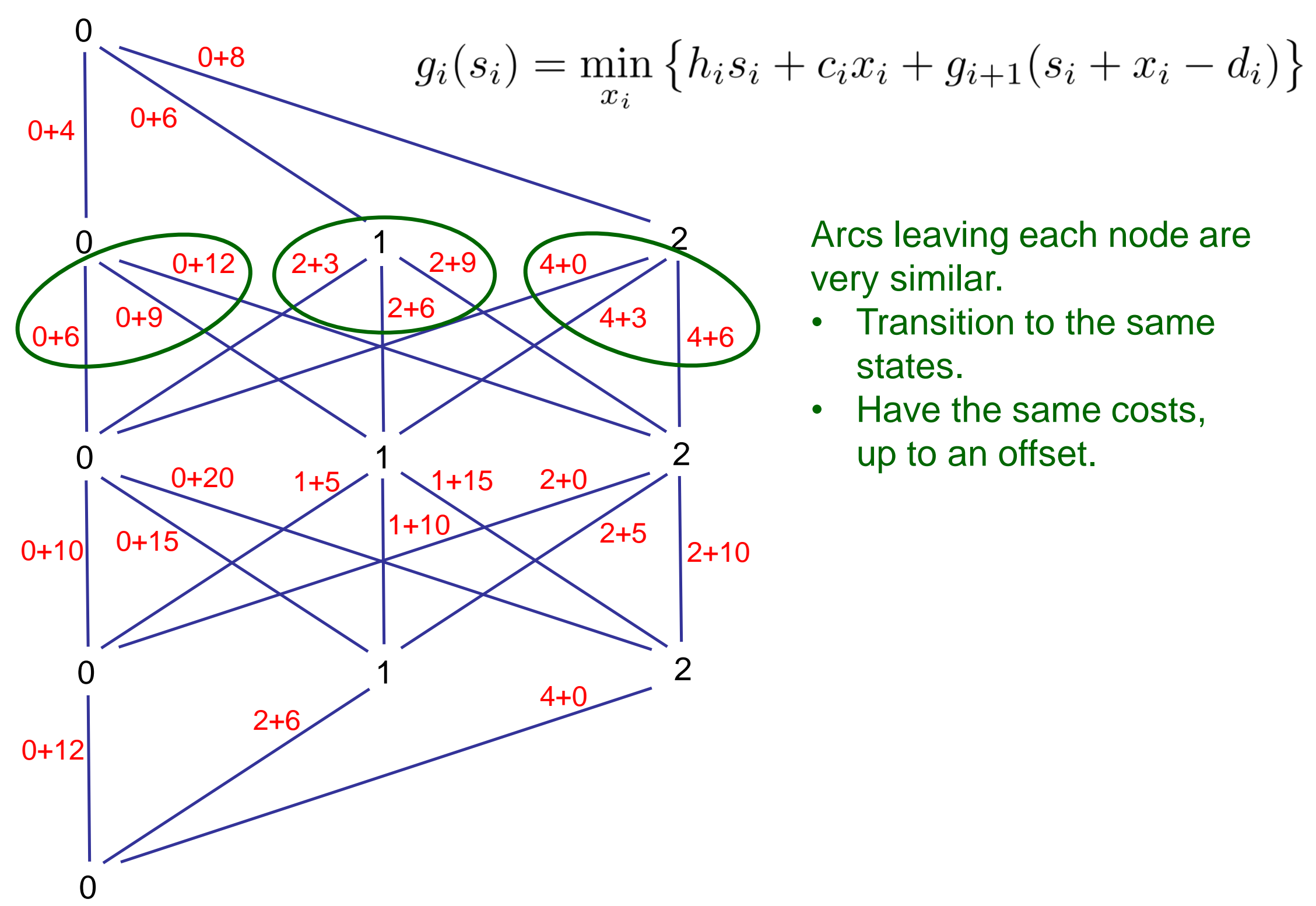




\section{Inventory Problem}

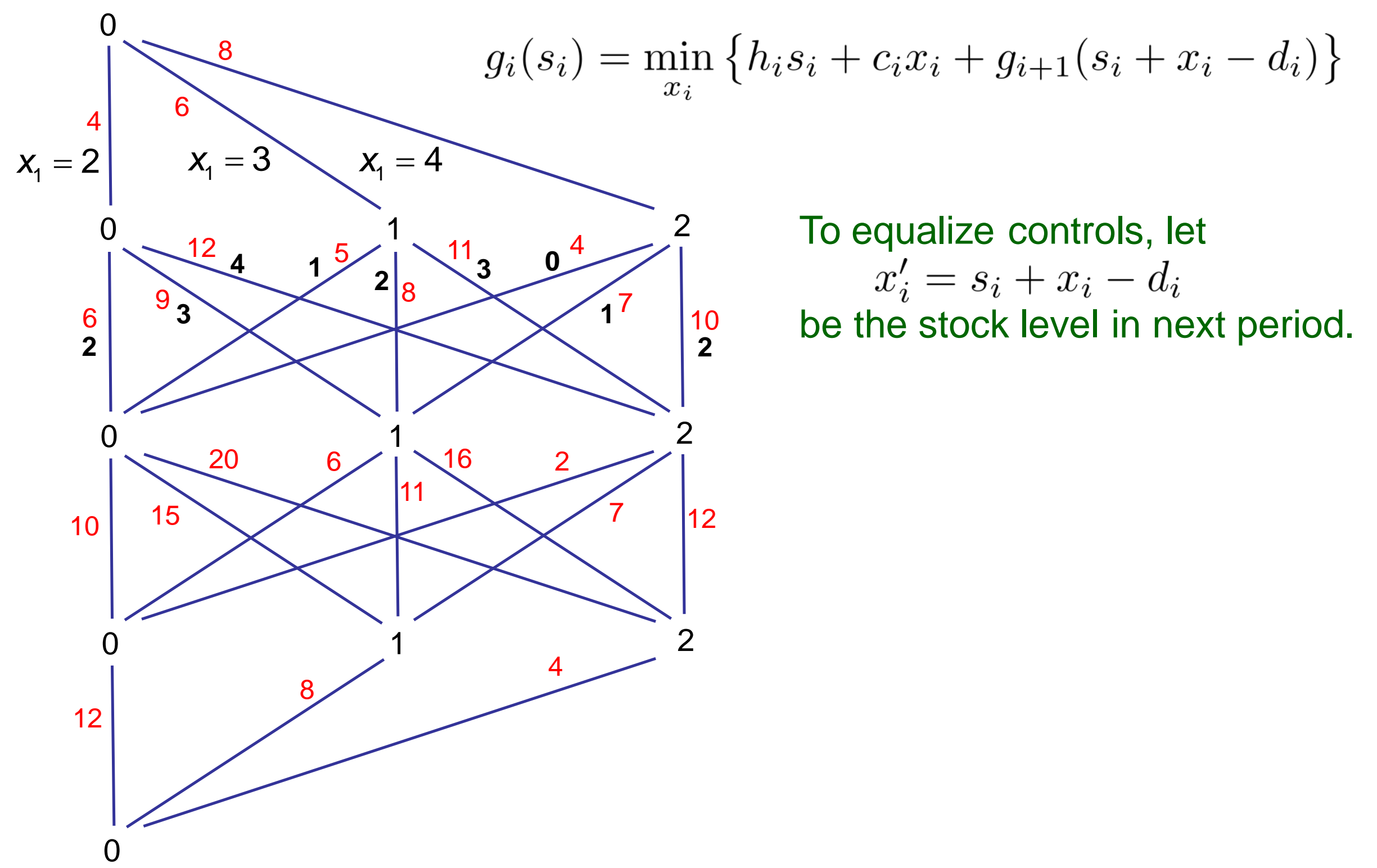




\section{Inventory Problem}

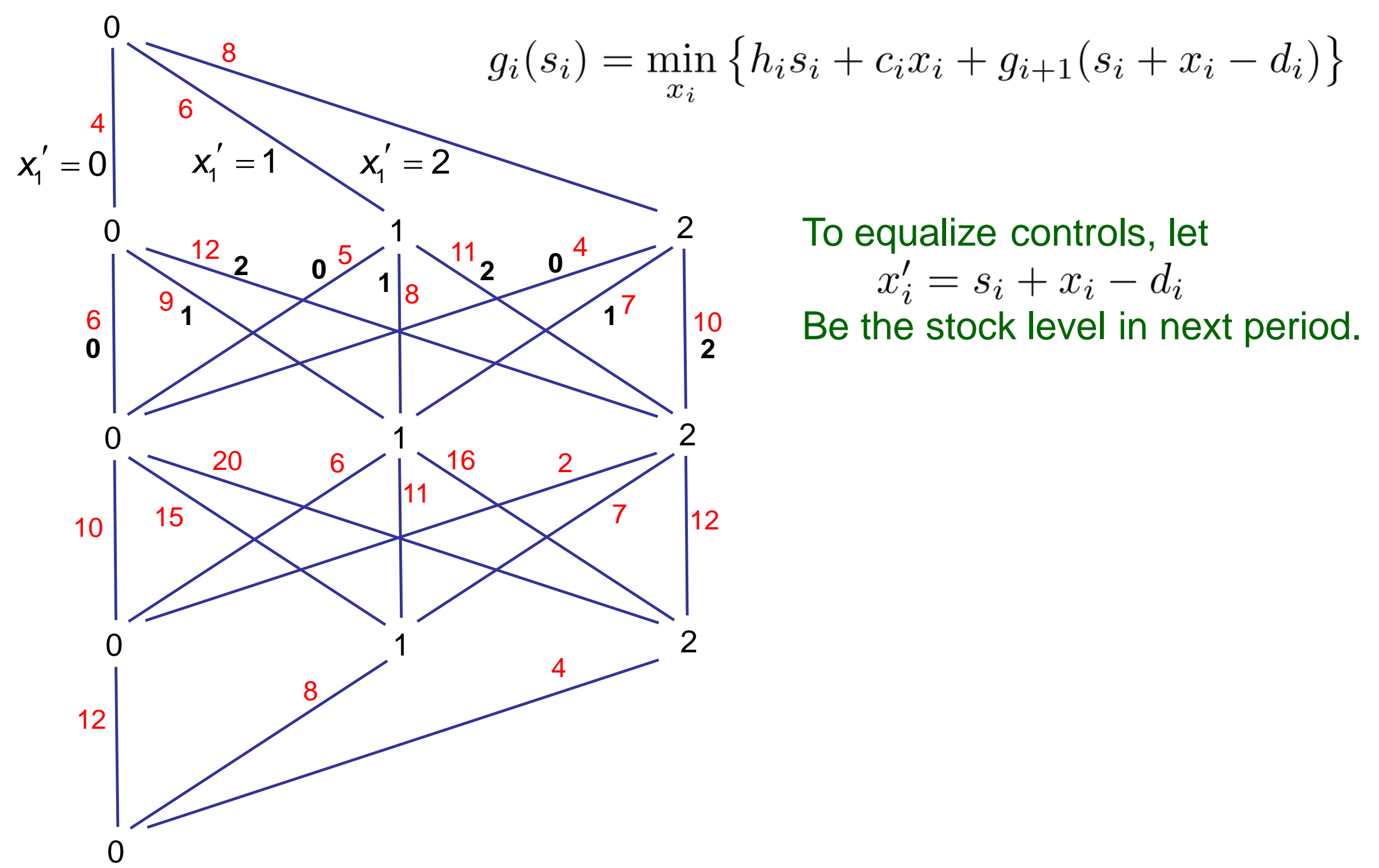




\section{Inventory Problem}

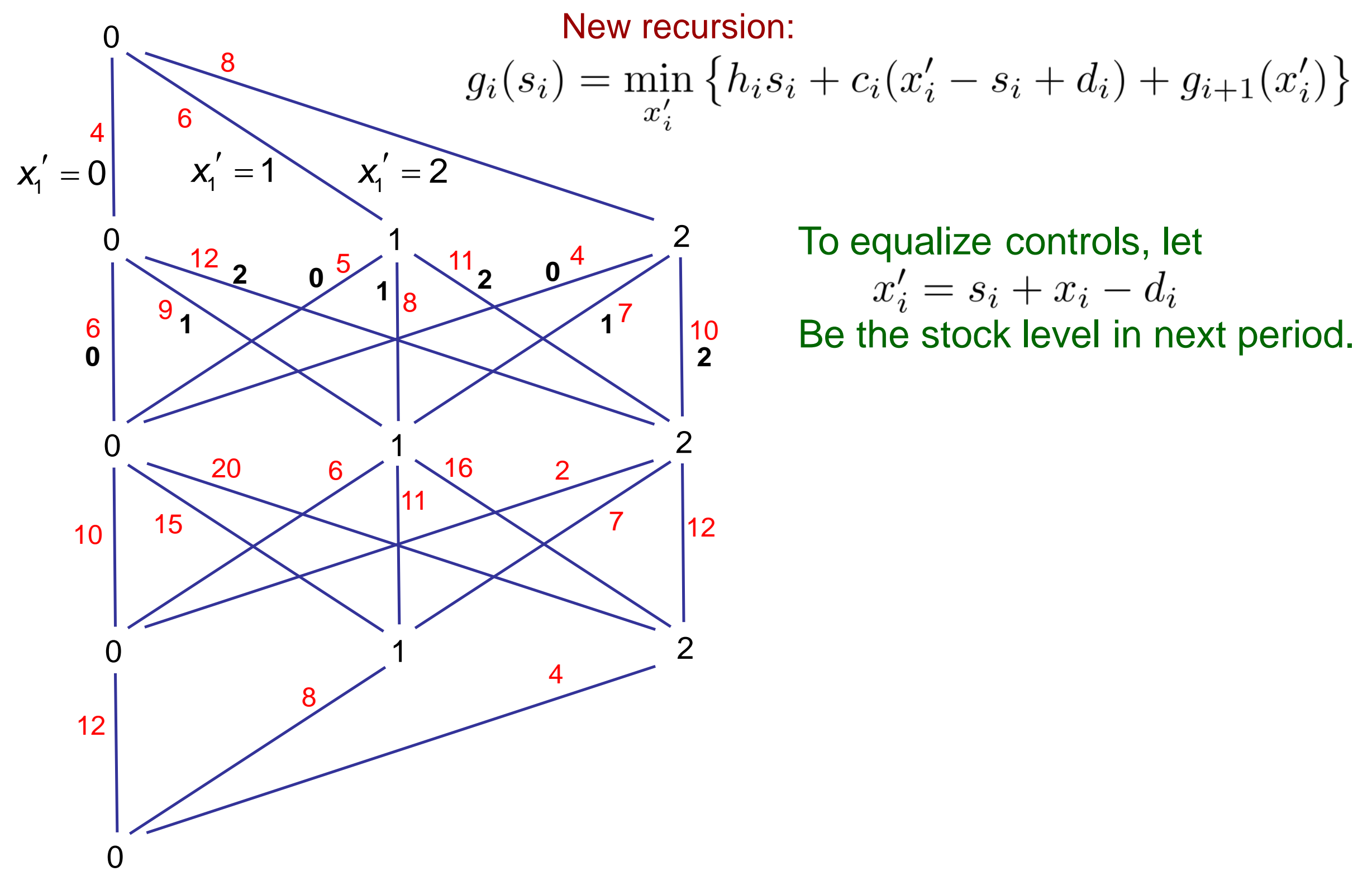




\section{Inventory Problem}

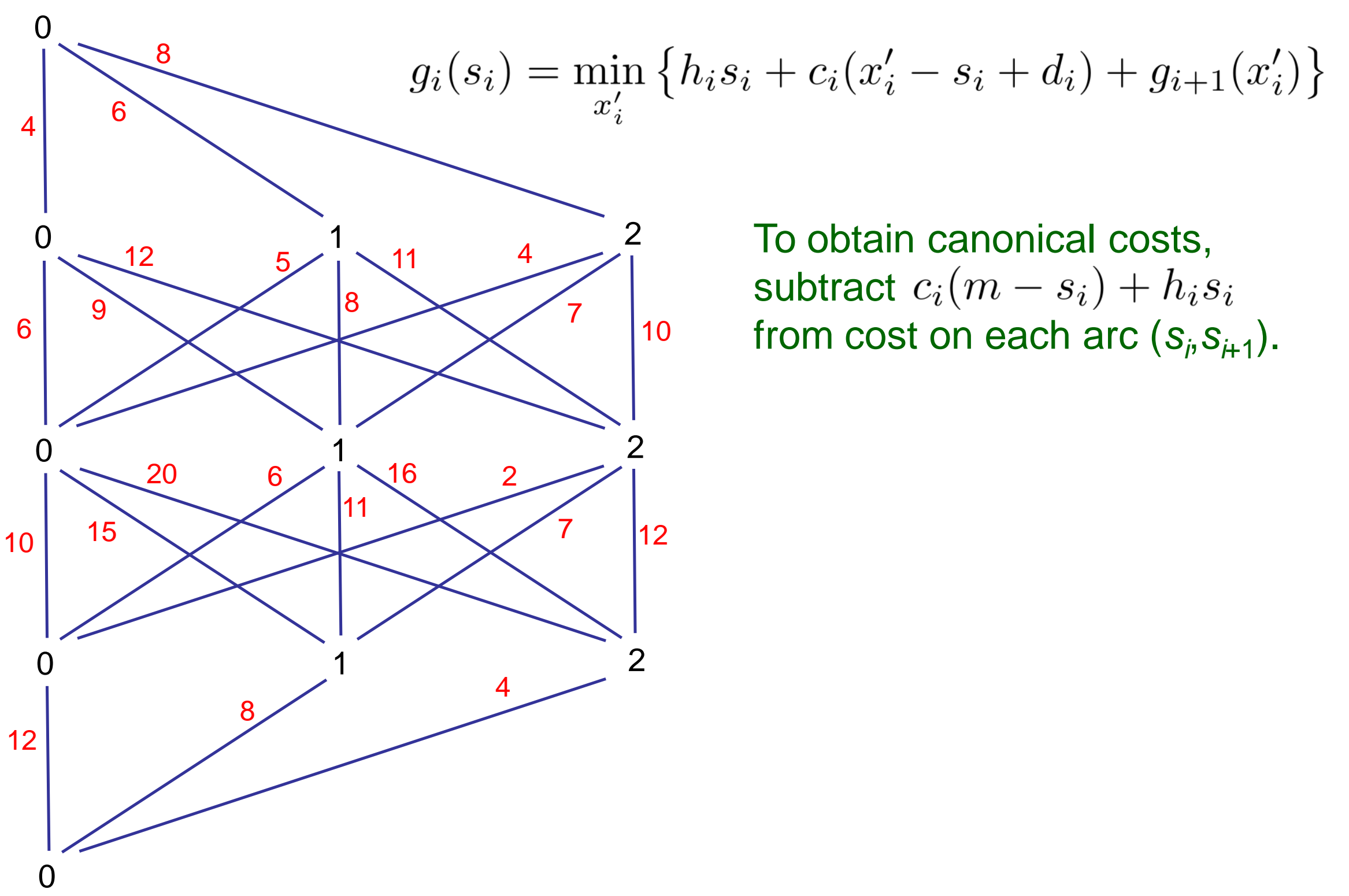




\section{Inventory Problem}

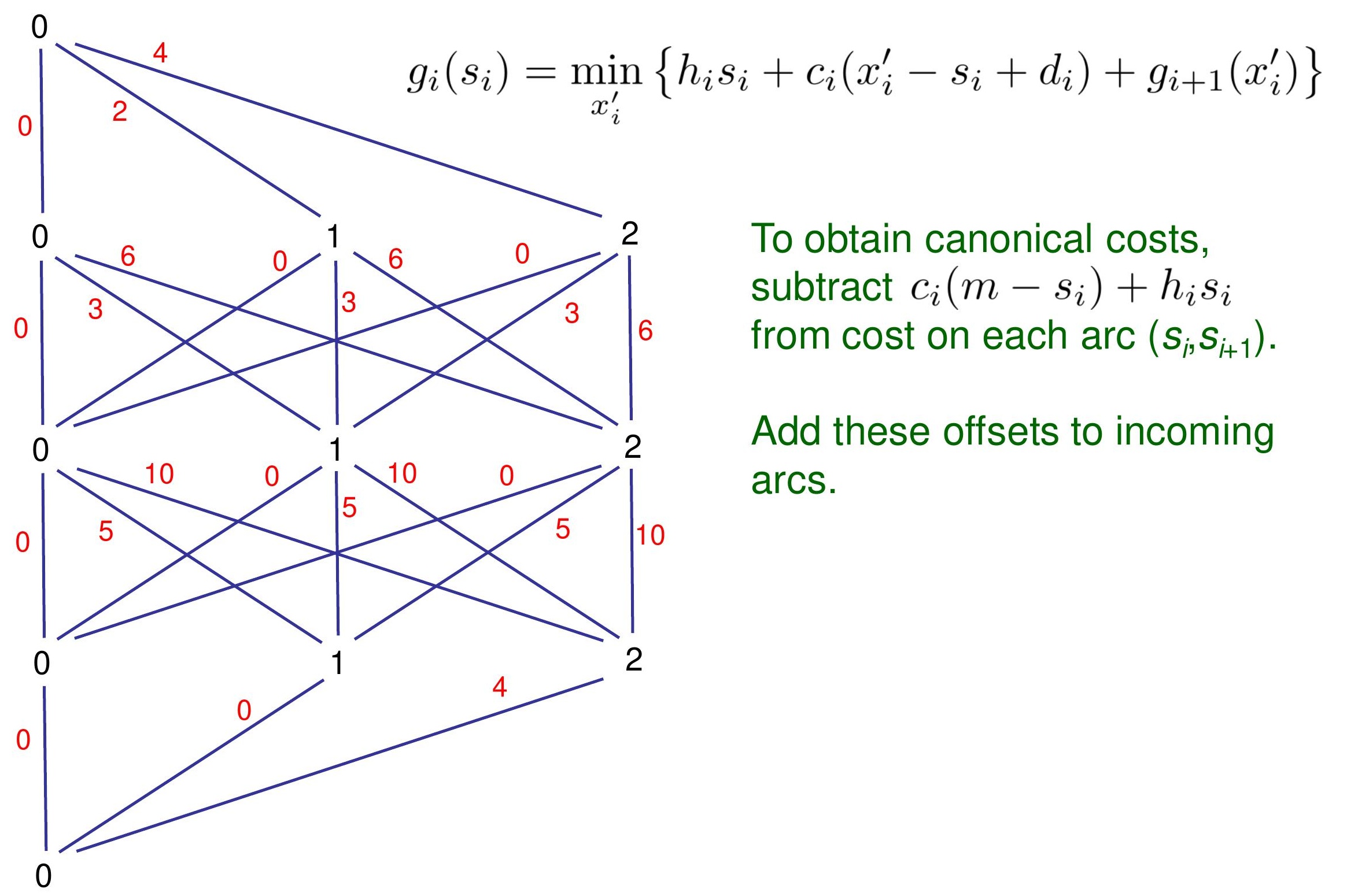




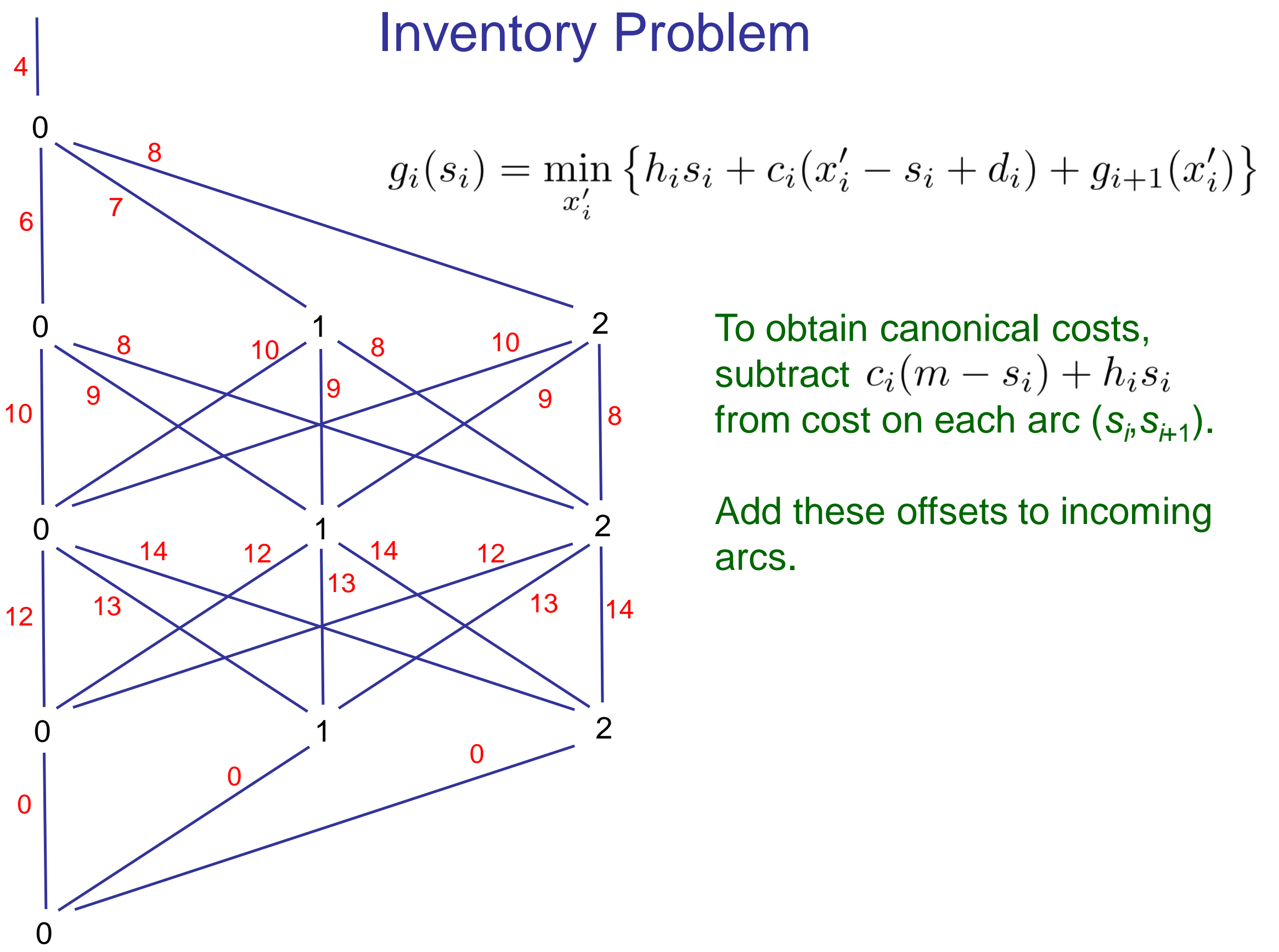




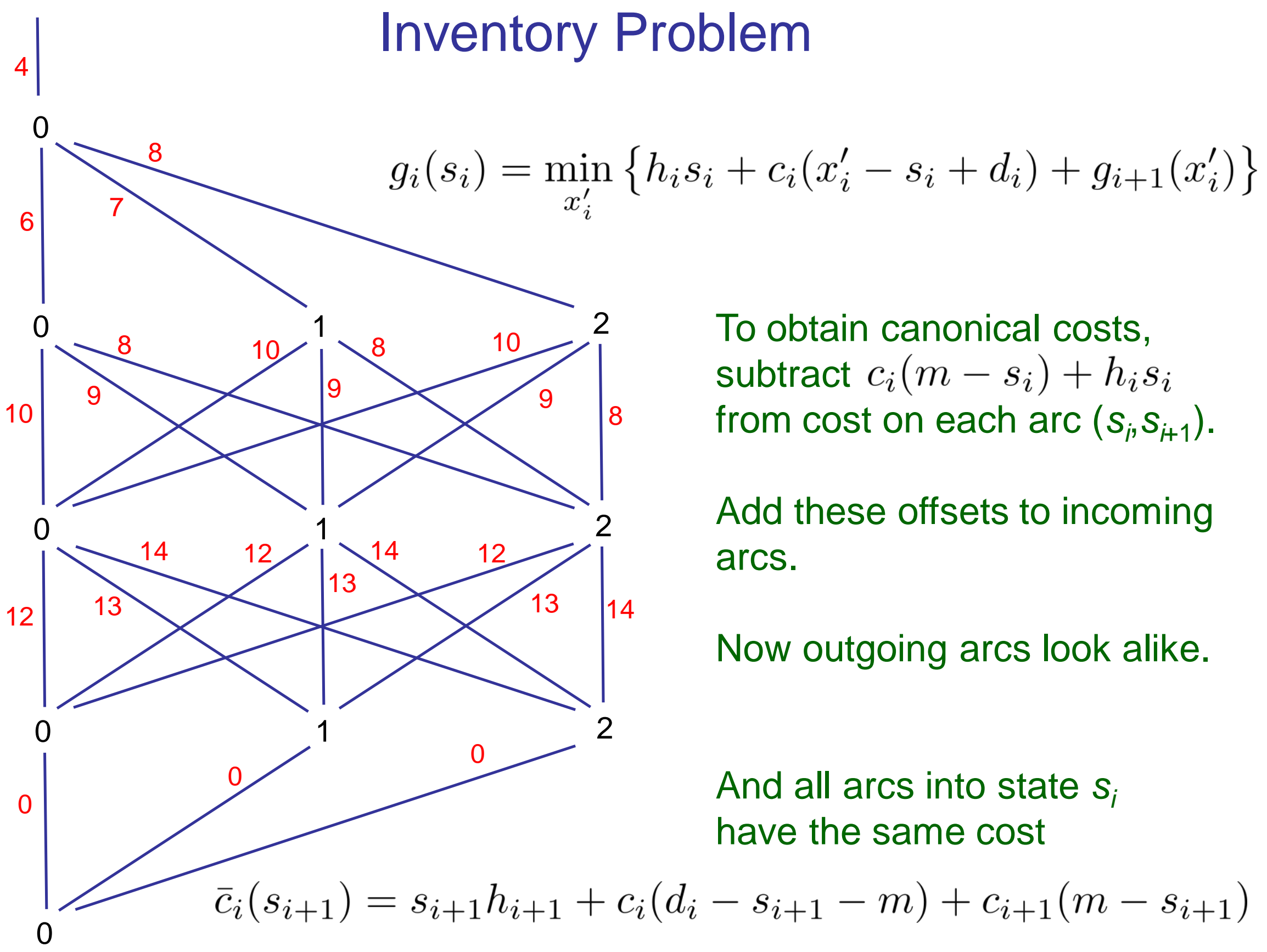




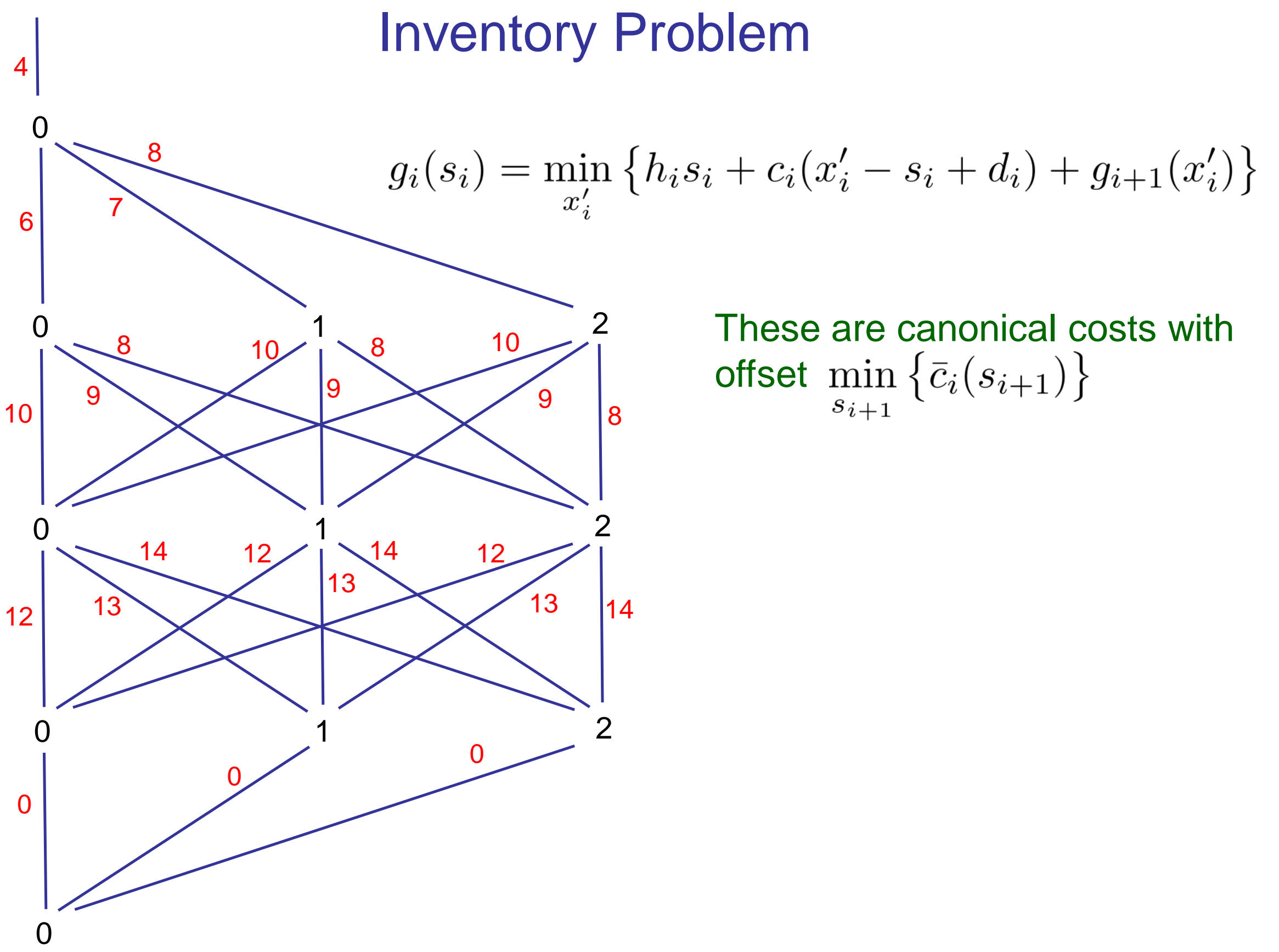




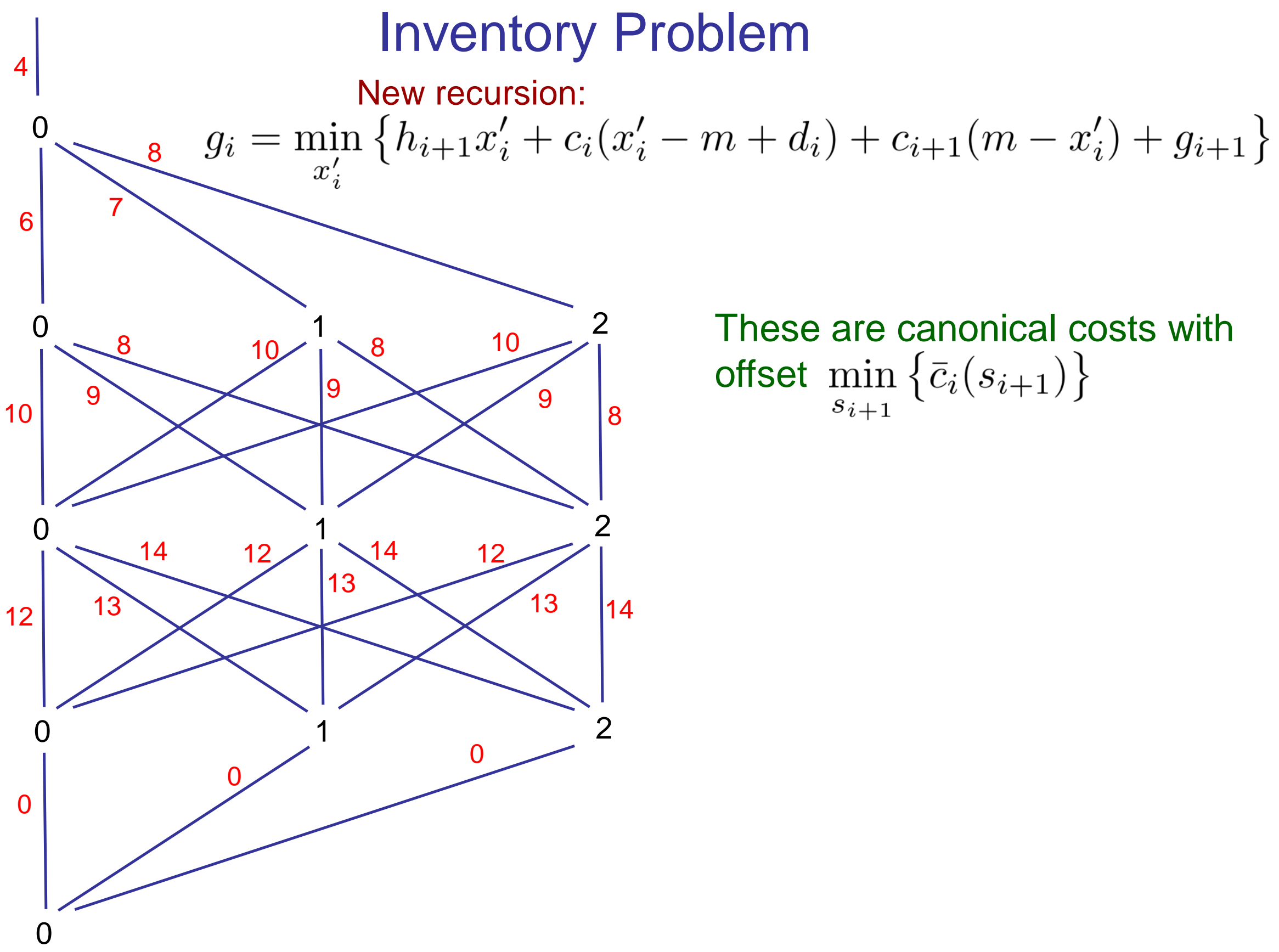




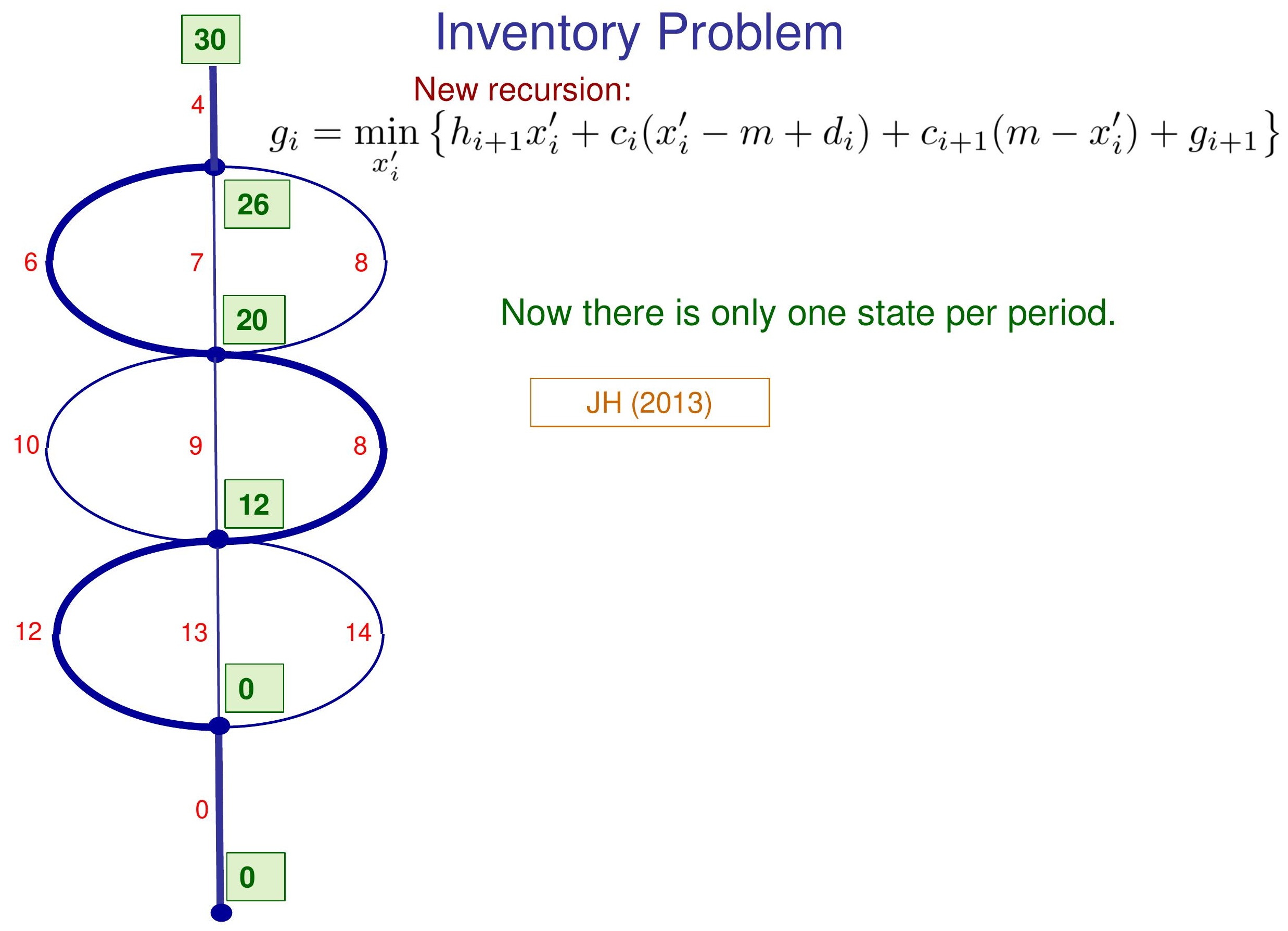




\section{Nonserial Decision Diagrams}

- Analogous to nonserial dynamic programming, independently(?) rediscovered many times:

- Nonserial DP (1972)

- Constraint satisfaction (1981)

- Data base queries (1983)

- k-trees (1985)

- Belief logics (1986)

- Bucket elimination (1987)

- Bayesian networks (1988)

- Pseudoboolean optimization (1990)

- Location analysis (1994) 


\section{Set Partitioning example}

Find collection of sets that partition elements $A, B, C, D$

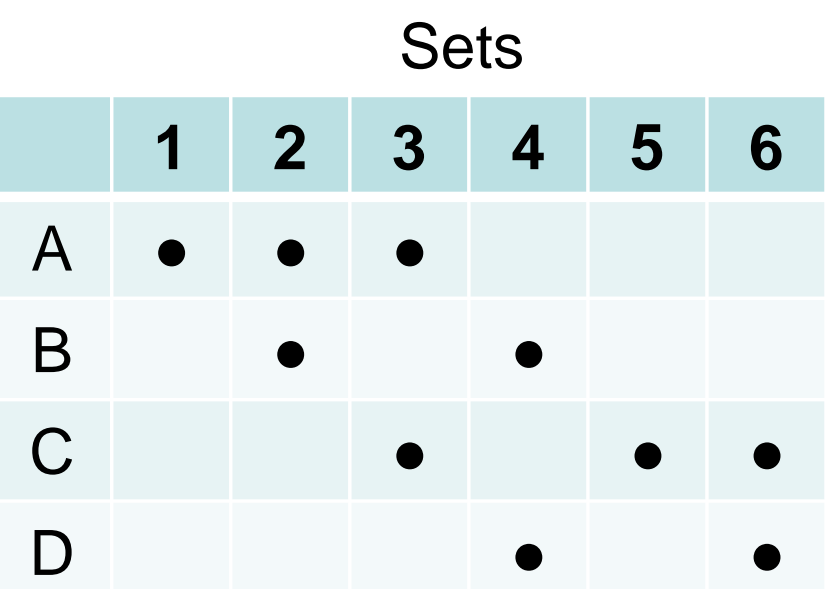




\section{Set Partitioning example}

Find collection of sets that partition elements $A, B, C, D$

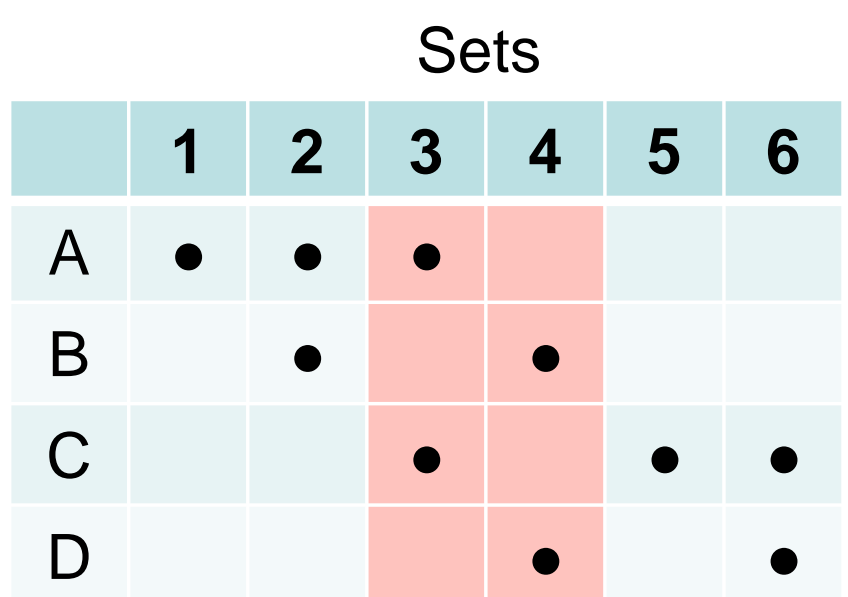

For example... 


\section{Set Partitioning example}

Find collection of sets that partition elements $A, B, C, D$

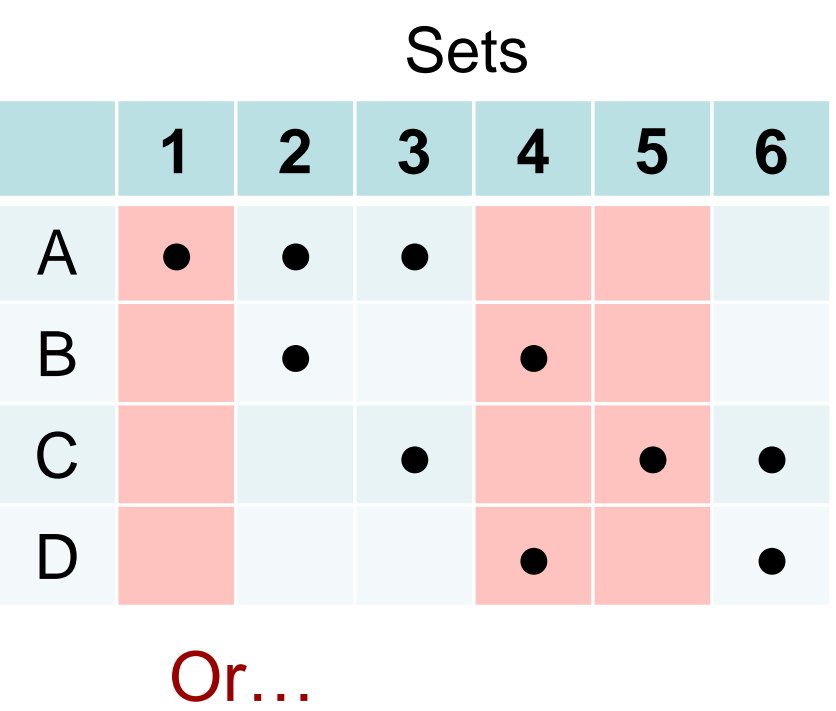




\section{Set Partitioning example}

Find collection of sets that partition elements A, B, C, D

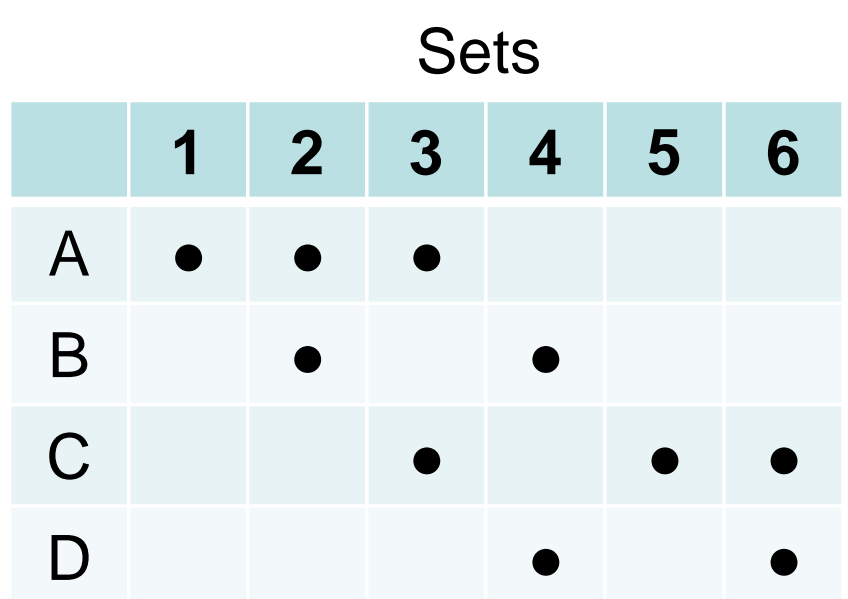

0-1 formulation

$$
\begin{aligned}
x_{1}+x_{2}+x_{3} & =1 \\
x_{2}+x_{4} & =1 \\
x_{3}+x_{5}+x_{6} & =1 \\
x_{4}+x_{6} & =1
\end{aligned}
$$




\section{Set Partitioning example}

Dependency graph

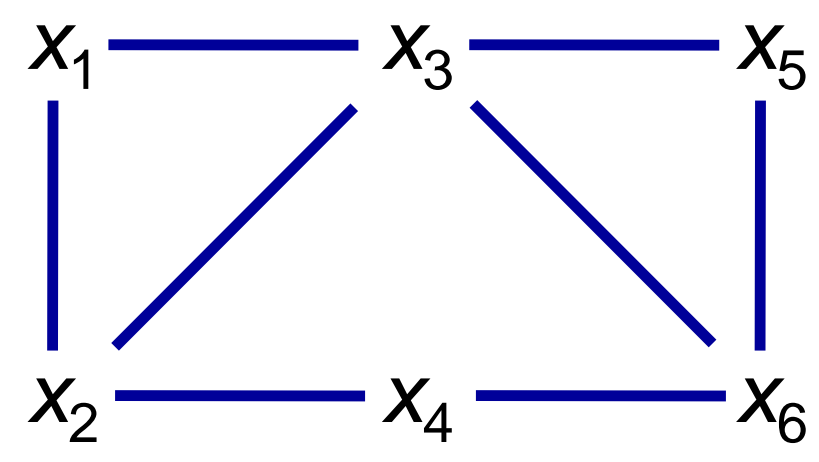

0-1 formulation

$$
\begin{aligned}
x_{1}+x_{2}+x_{3} & =1 \\
x_{2}+x_{4} & =1 \\
x_{3}+x_{5}+x_{6} & =1 \\
x_{4}+x_{6} & =1
\end{aligned}
$$

$$
x_{j}=1 \Rightarrow \text { set } j \text { selected }
$$




\section{Set Partitioning example}

Enumeration order

$x_{2}$

Dependency graph

$x_{3}$

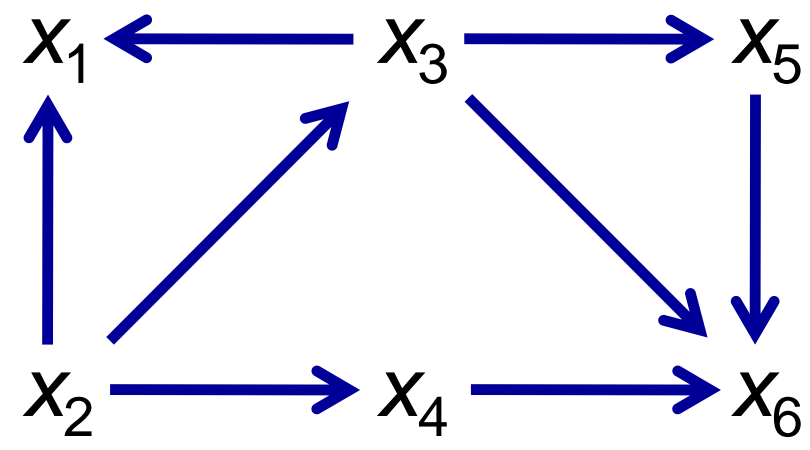

$x_{4}$

$x_{1}$

$x_{5}$

$x_{6}$ 


\section{Set Partitioning example}

Enumeration order

$x_{2}$

Dependency graph
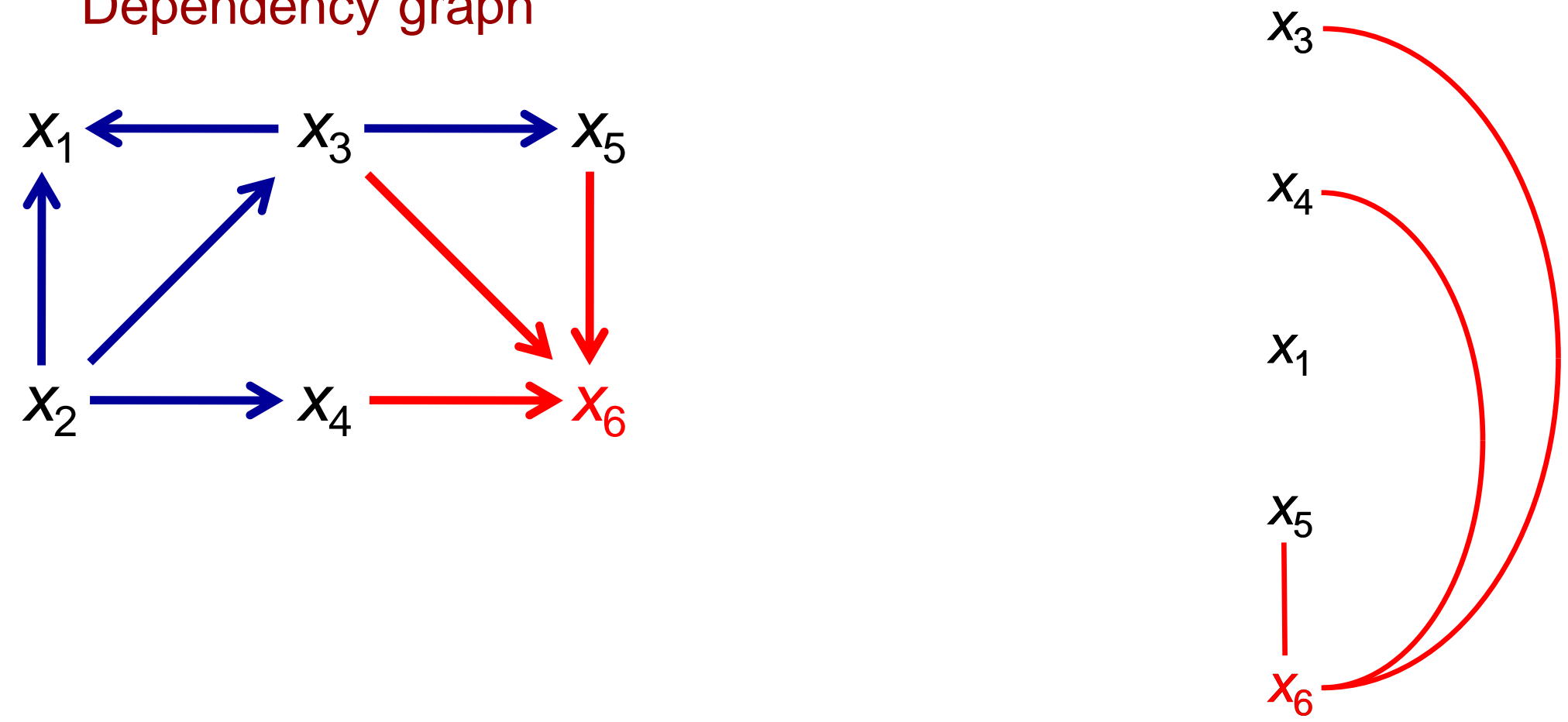


\section{Set Partitioning example}

Enumeration order

$x_{2}$

Dependency graph
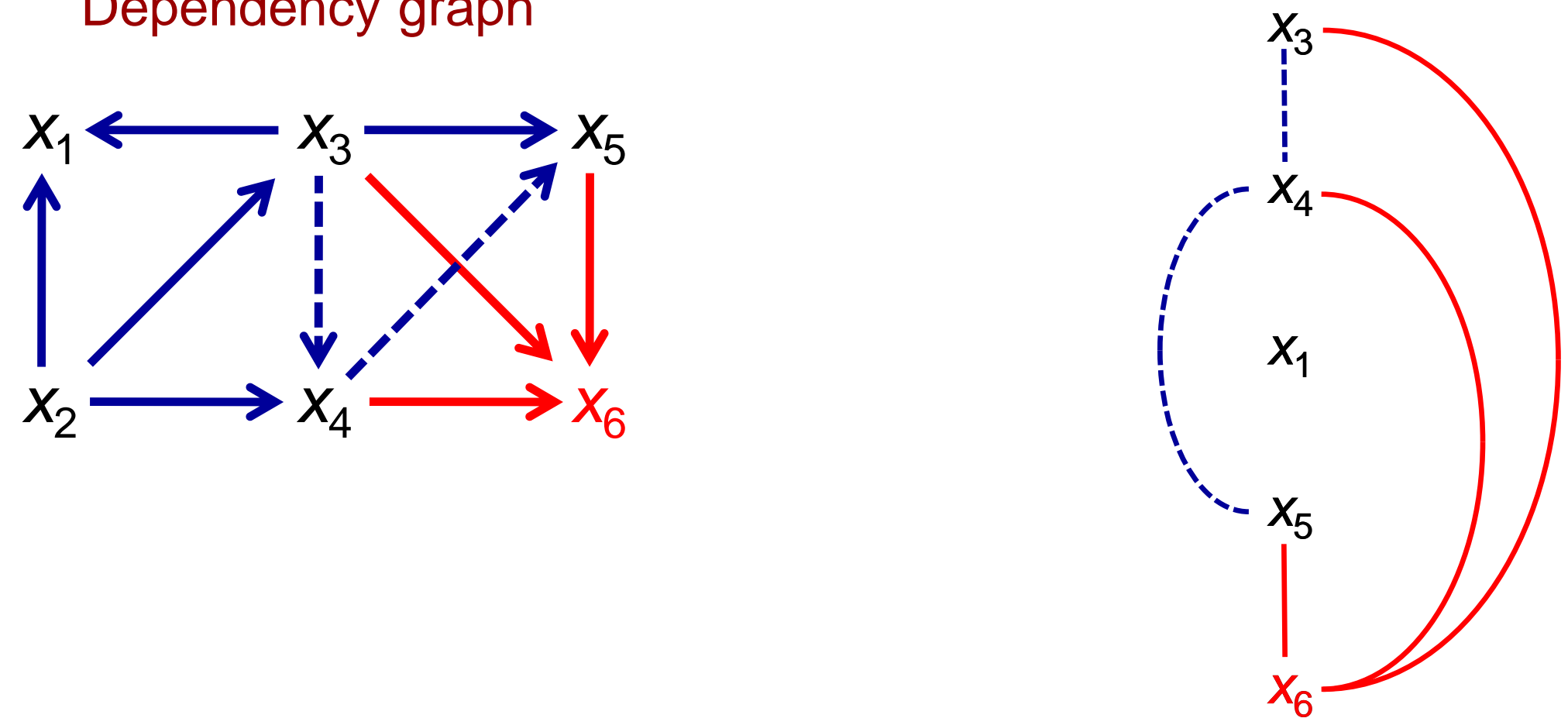


\section{Set Partitioning example}

Enumeration order

$x_{2}$

Dependency graph
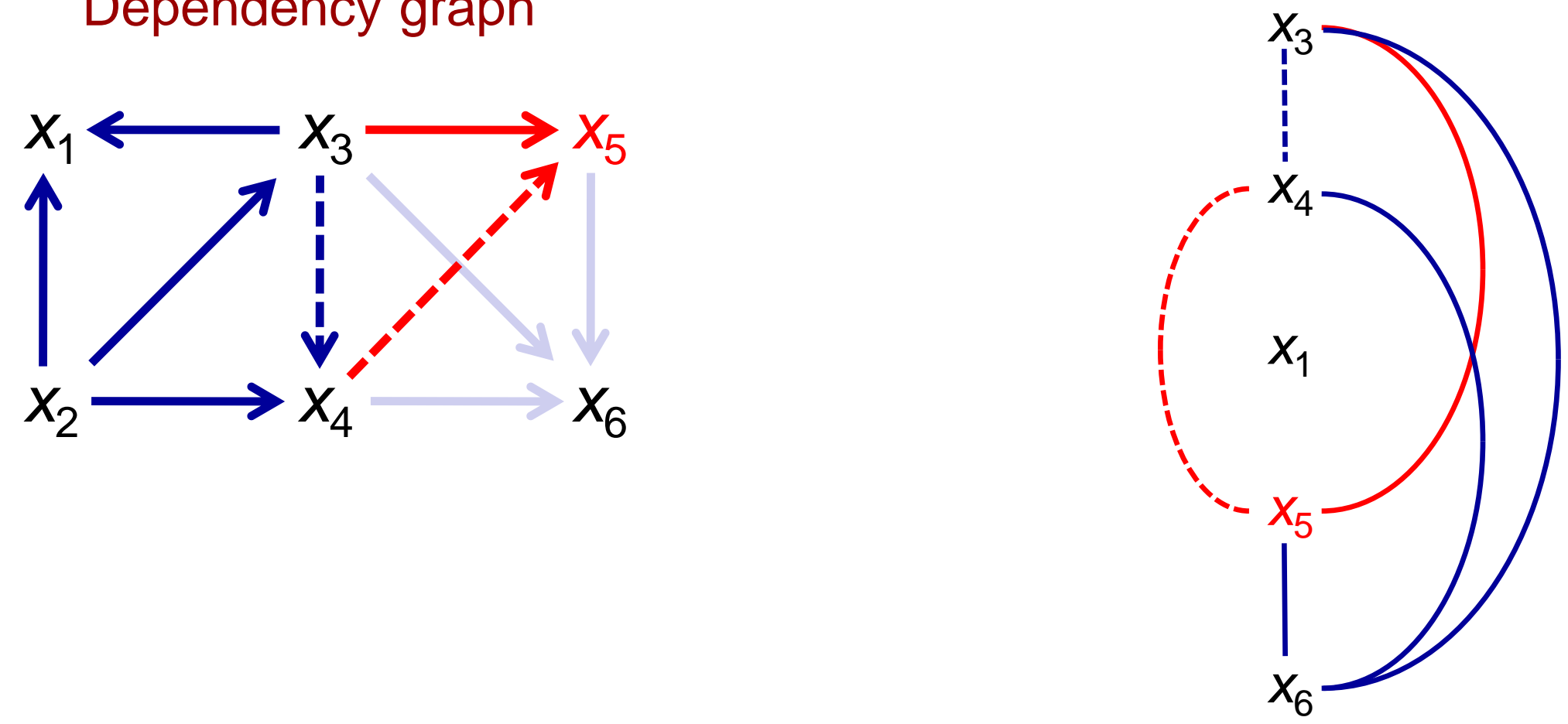


\section{Set Partitioning example}

Dependency graph

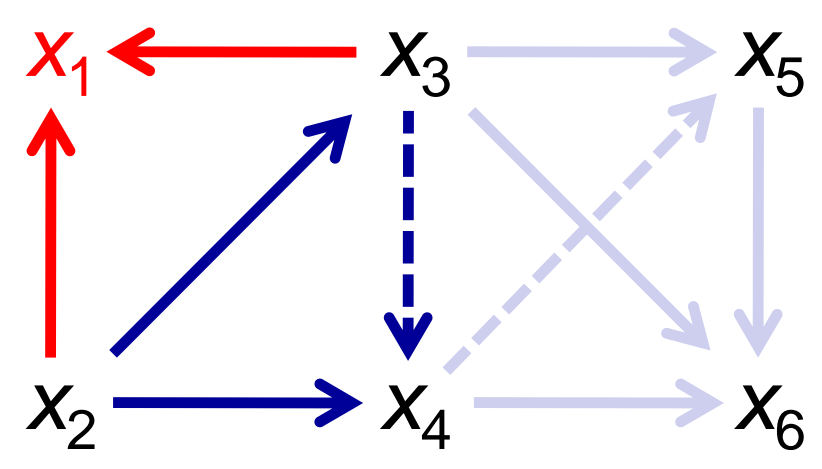

Enumeration order

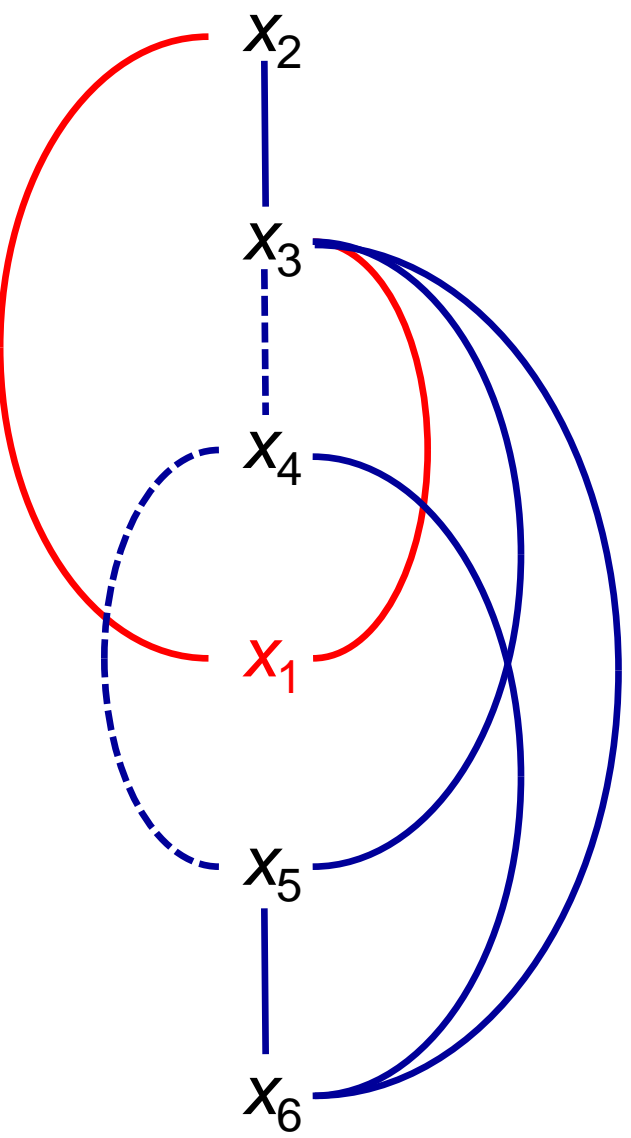




\section{Set Partitioning example}

Dependency graph

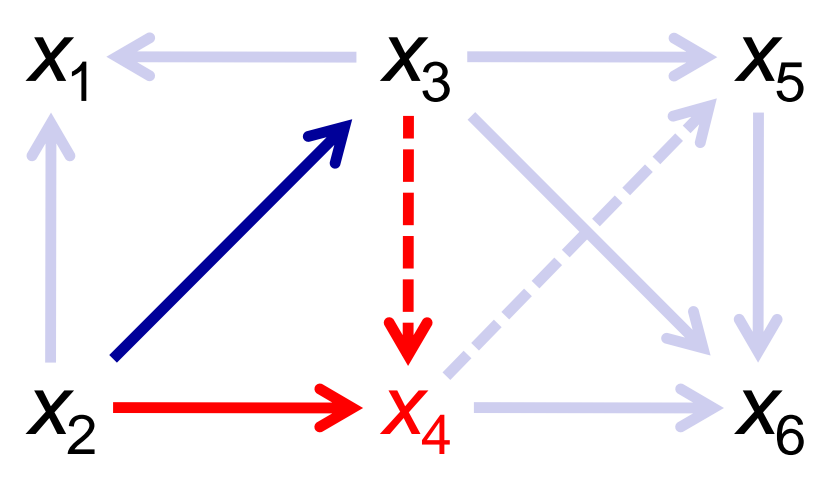

Enumeration order

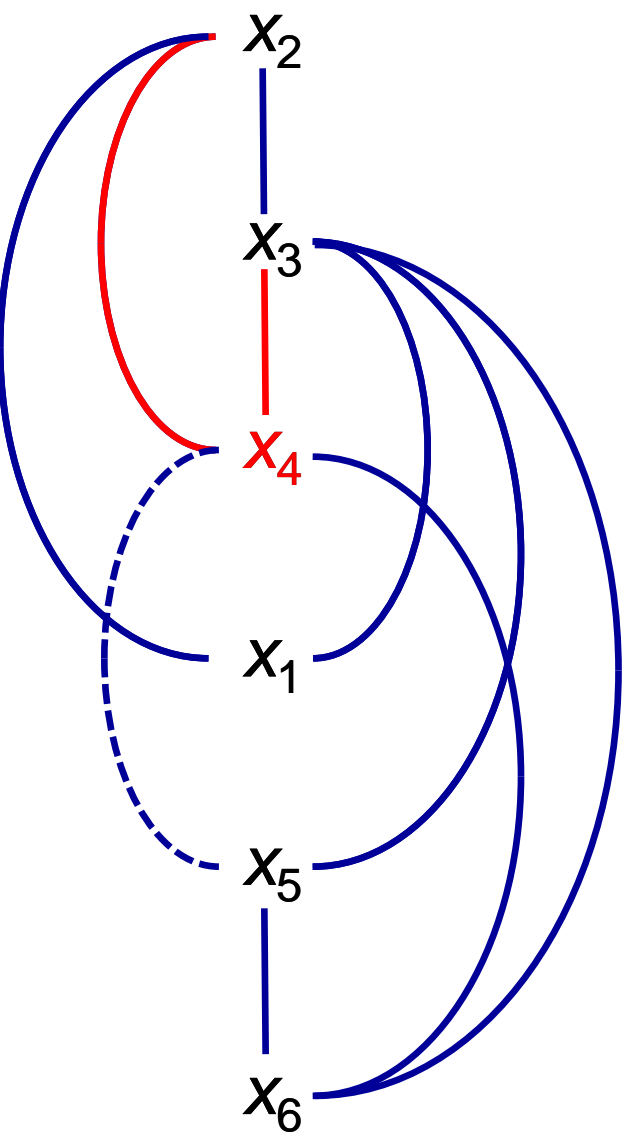




\section{Set Partitioning example}

Dependency graph

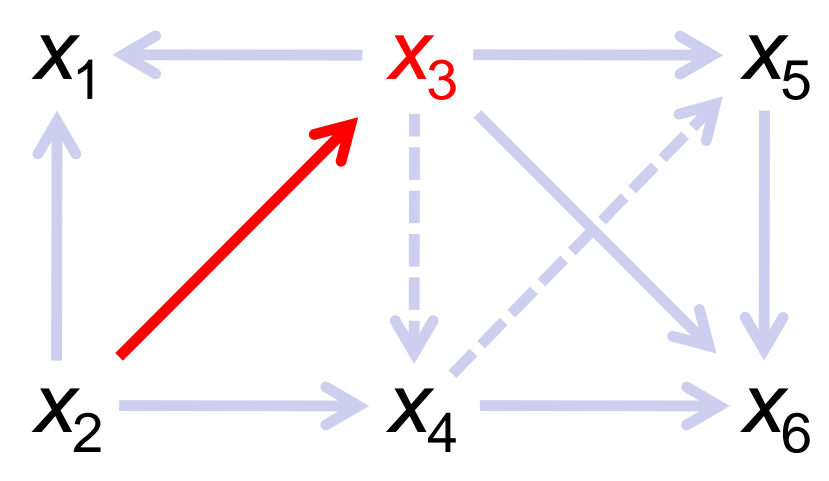

Enumeration order

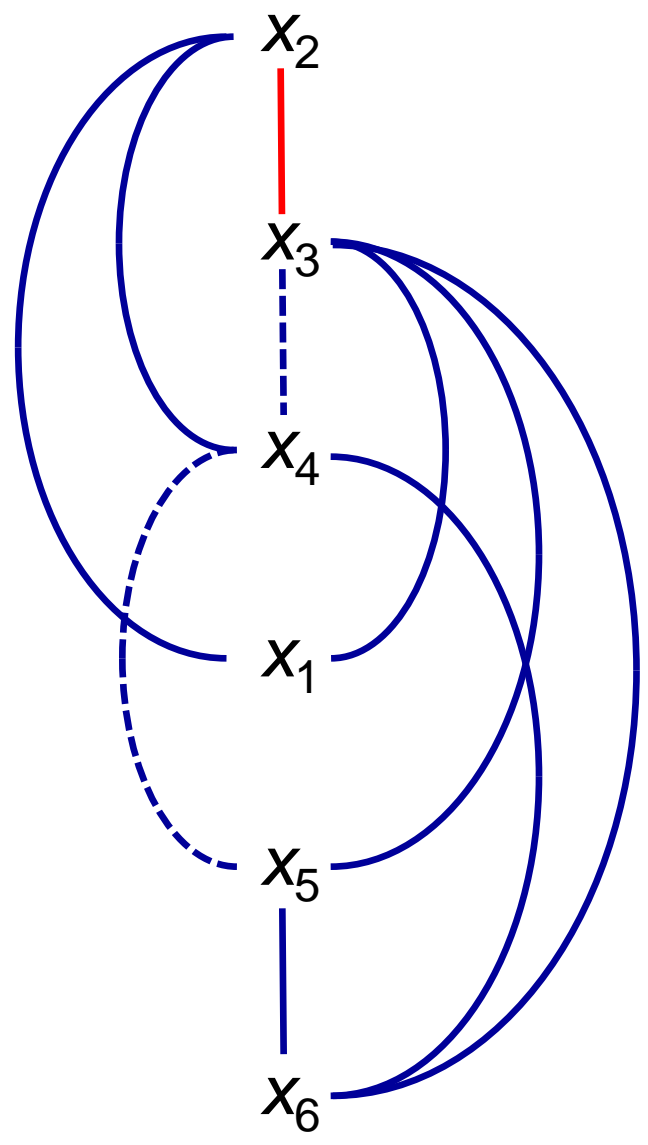




\section{Set Partitioning example}

Dependency graph

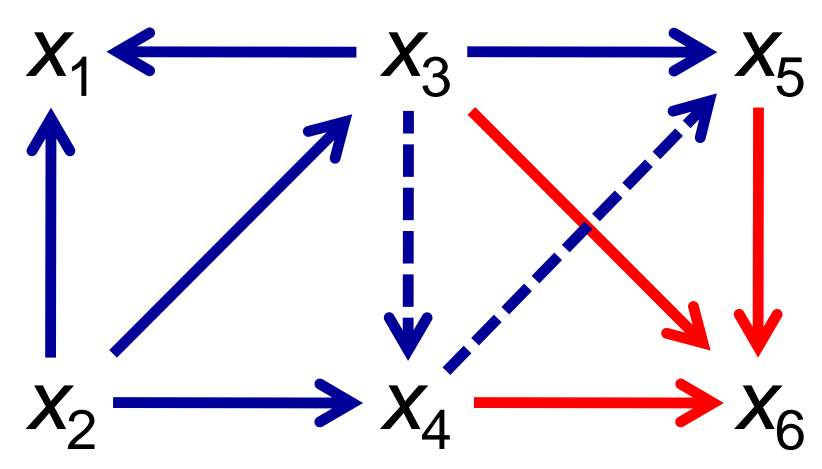

Enumeration order

Induced width $=3$

(max in-degree)

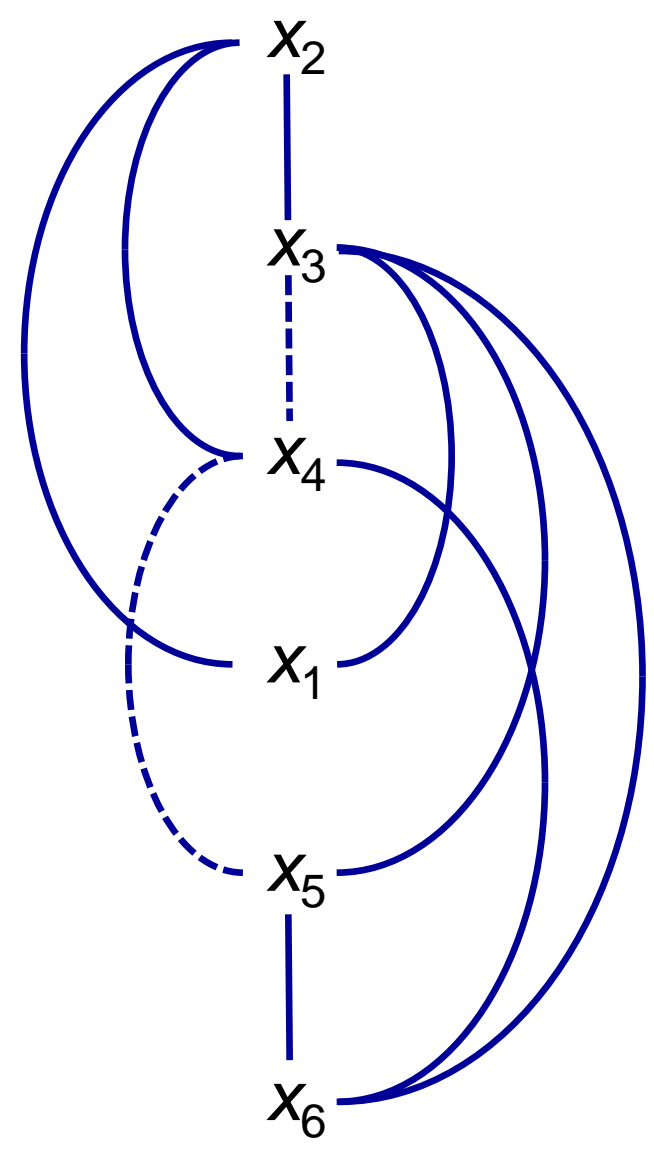




\section{Set Partitioning example}

Solution by nonserial DP

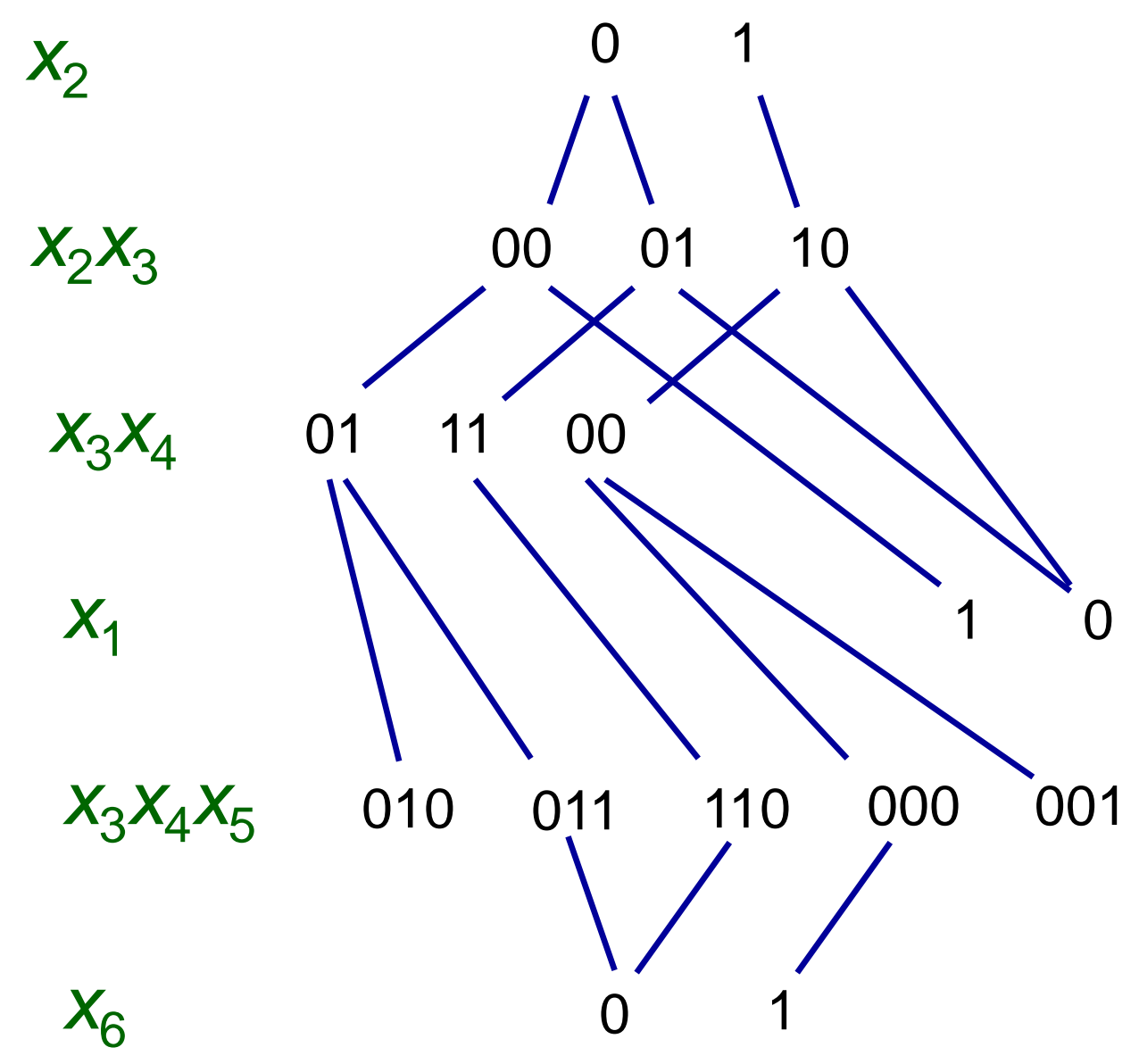

Enumeration order

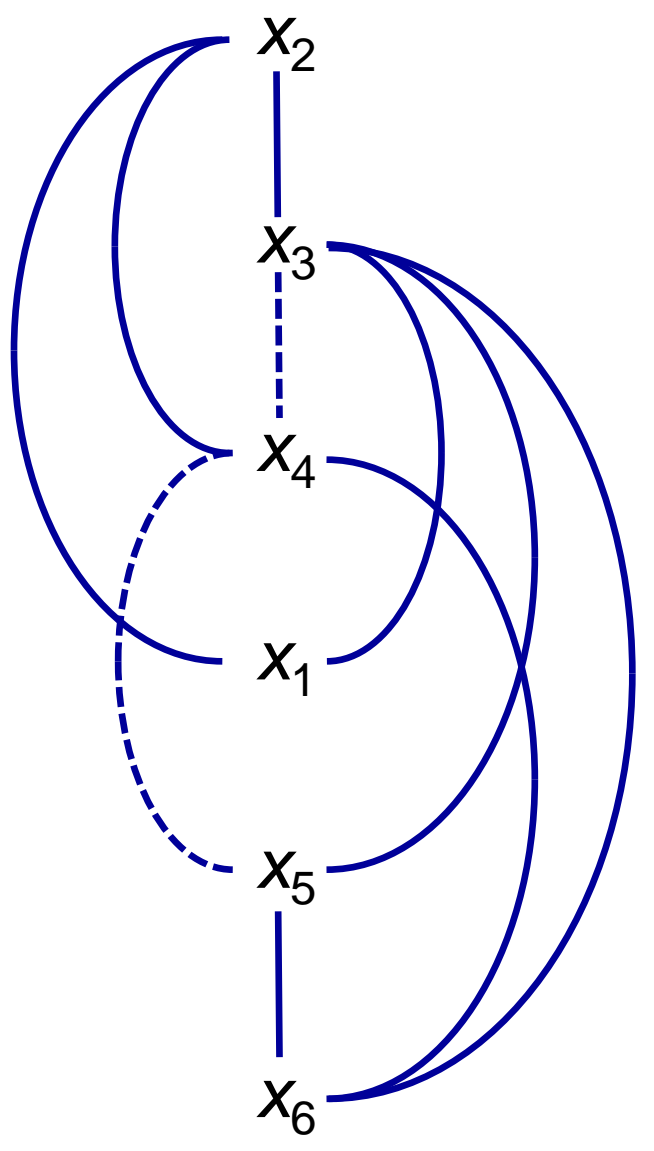




\section{Set Partitioning example}

Solution by nonserial DP

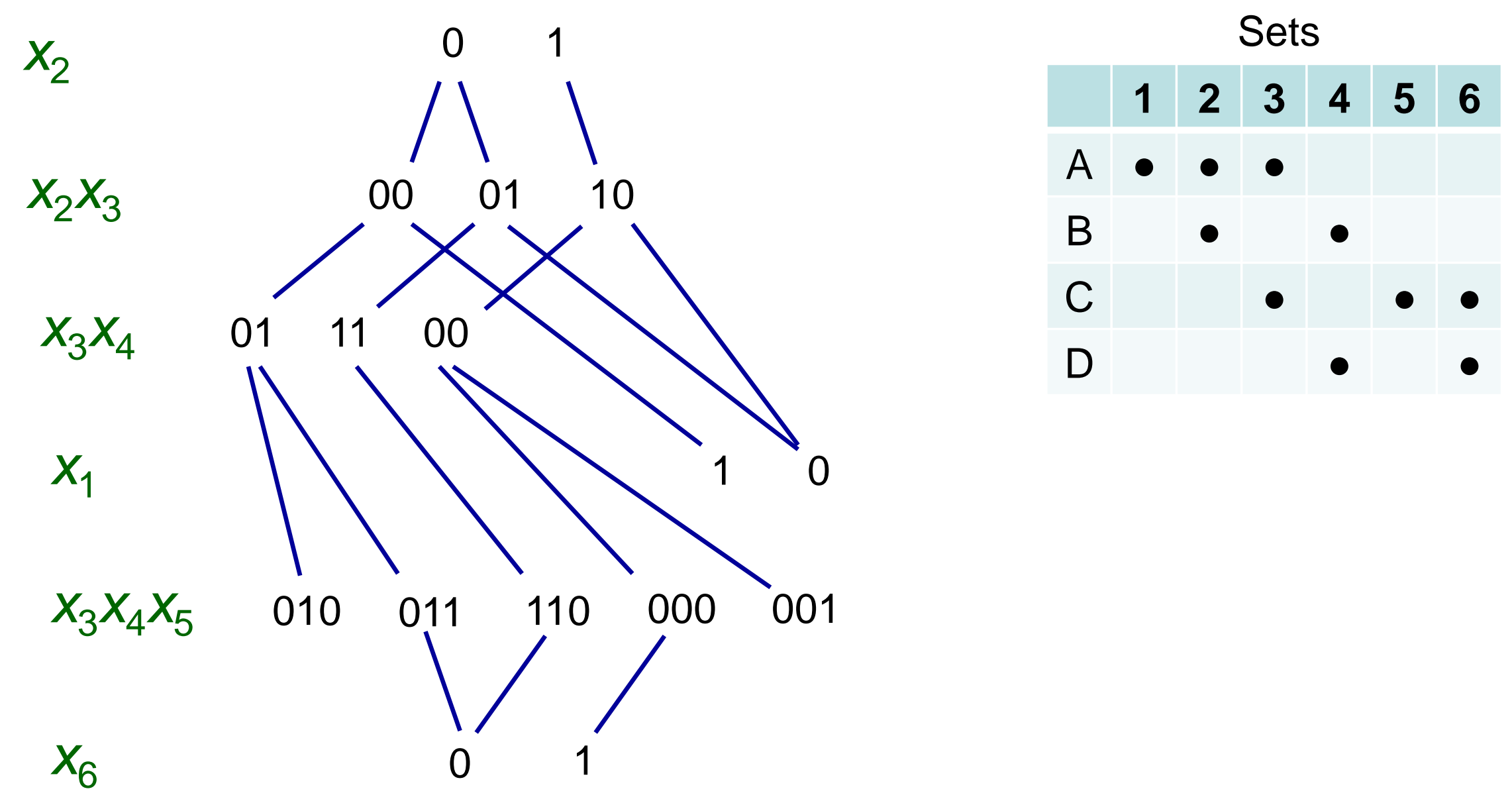




\section{Set Partitioning example}

Feasible solution

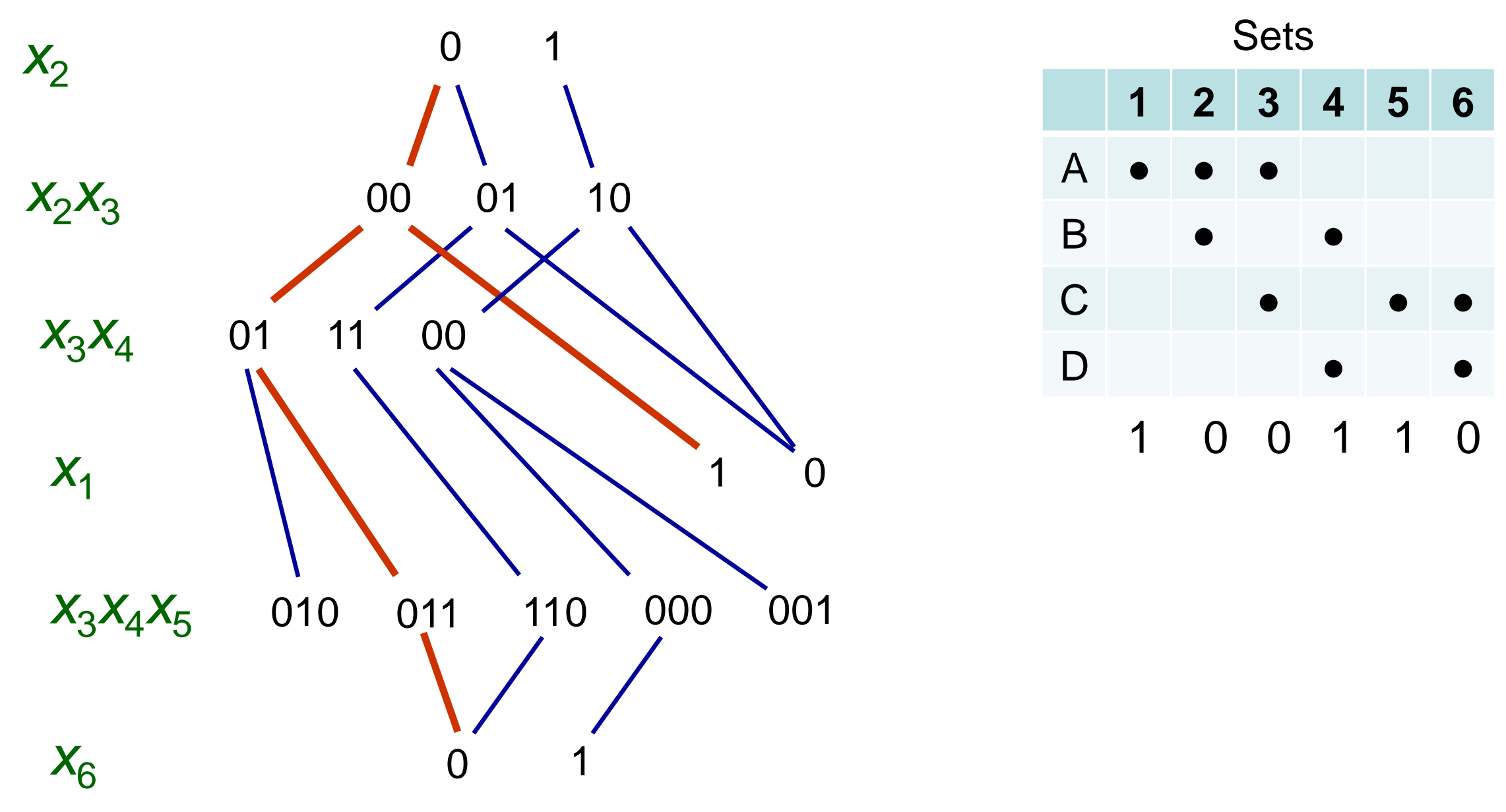




\section{Set Partitioning example}

Feasible solution

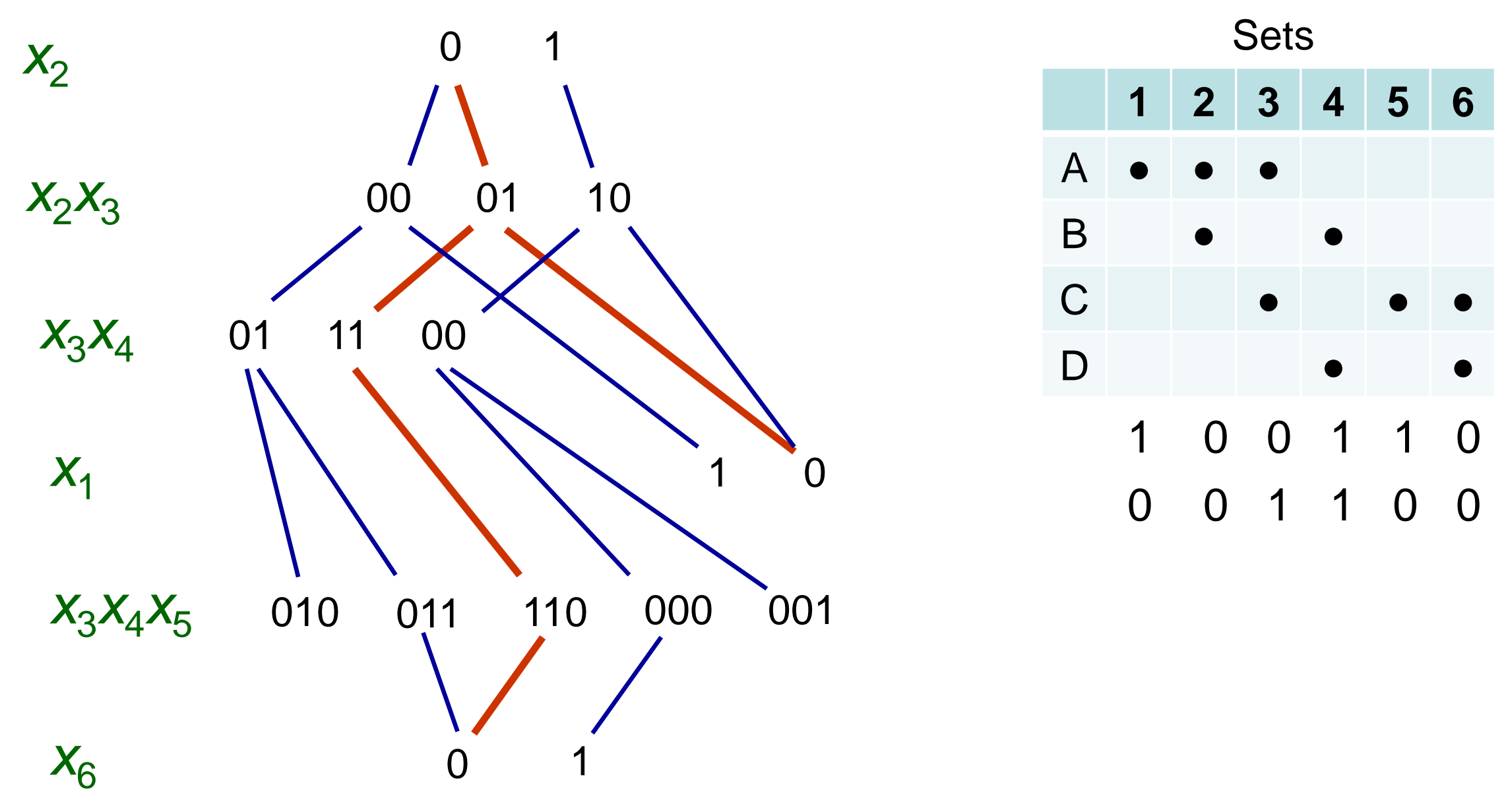




\section{Set Partitioning example}

Feasible solution

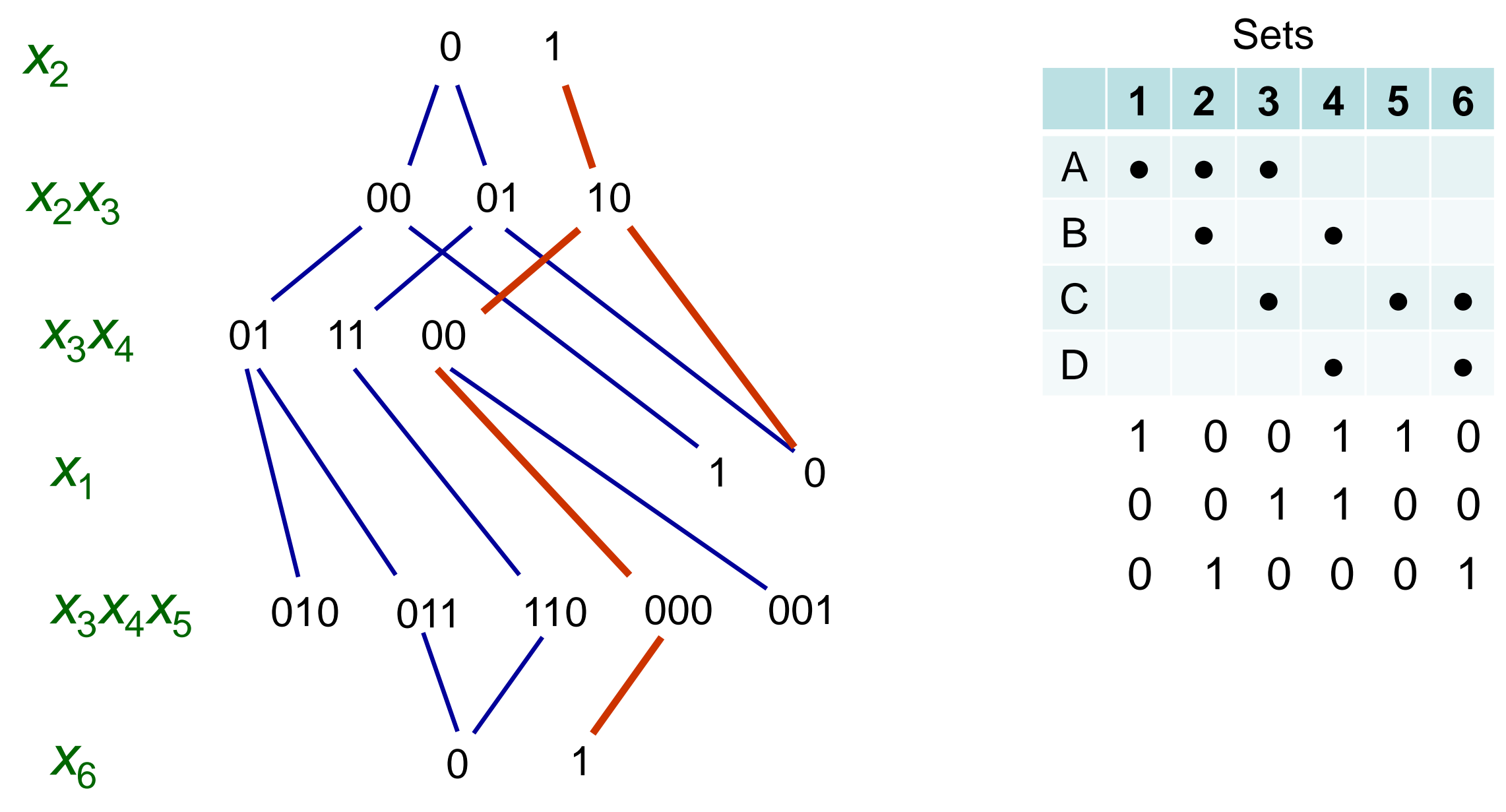




\section{Set Partitioning example}

Solution by nonserial DP

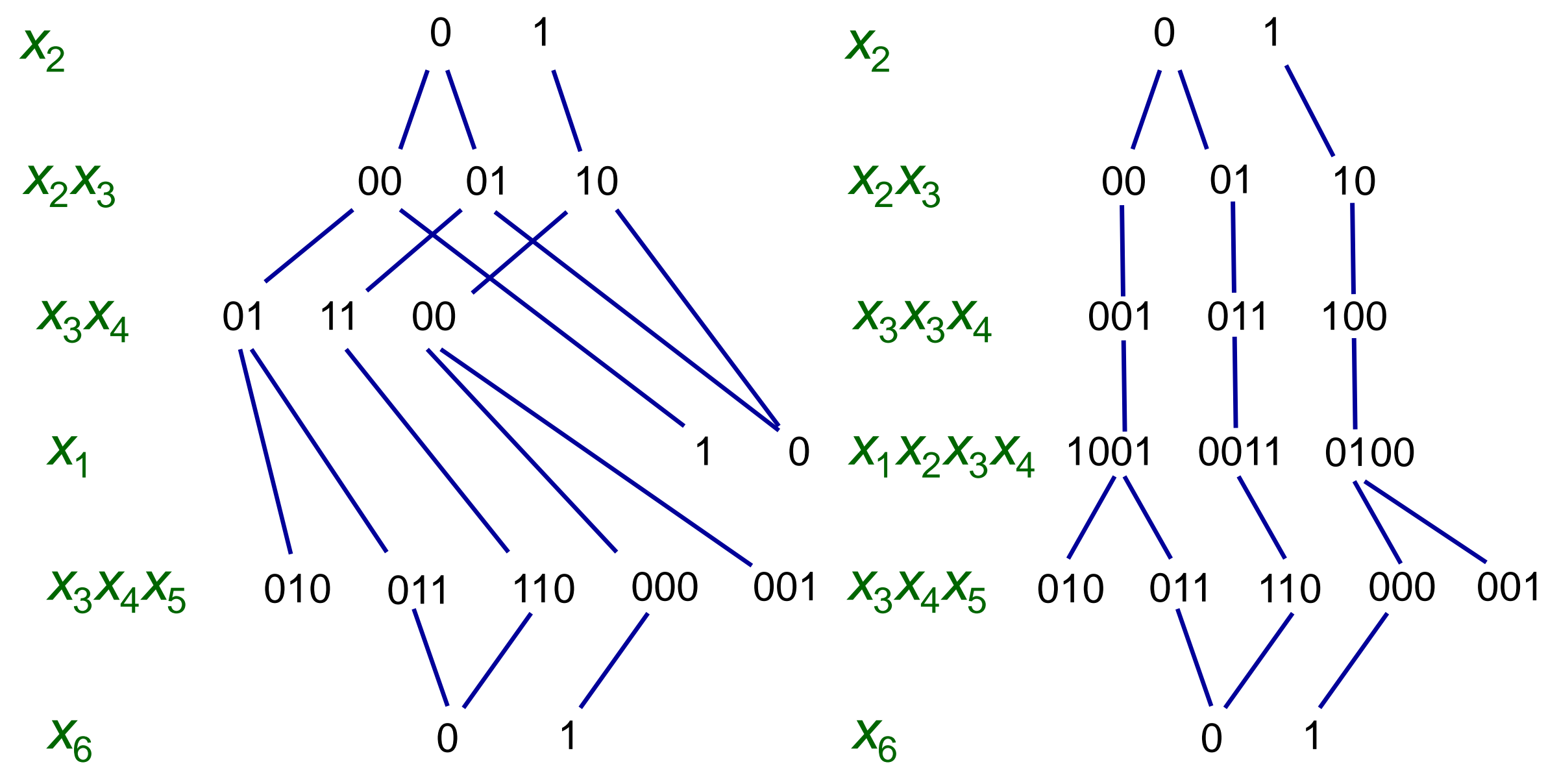




\section{Set Partitioning example}

Feasible solutions

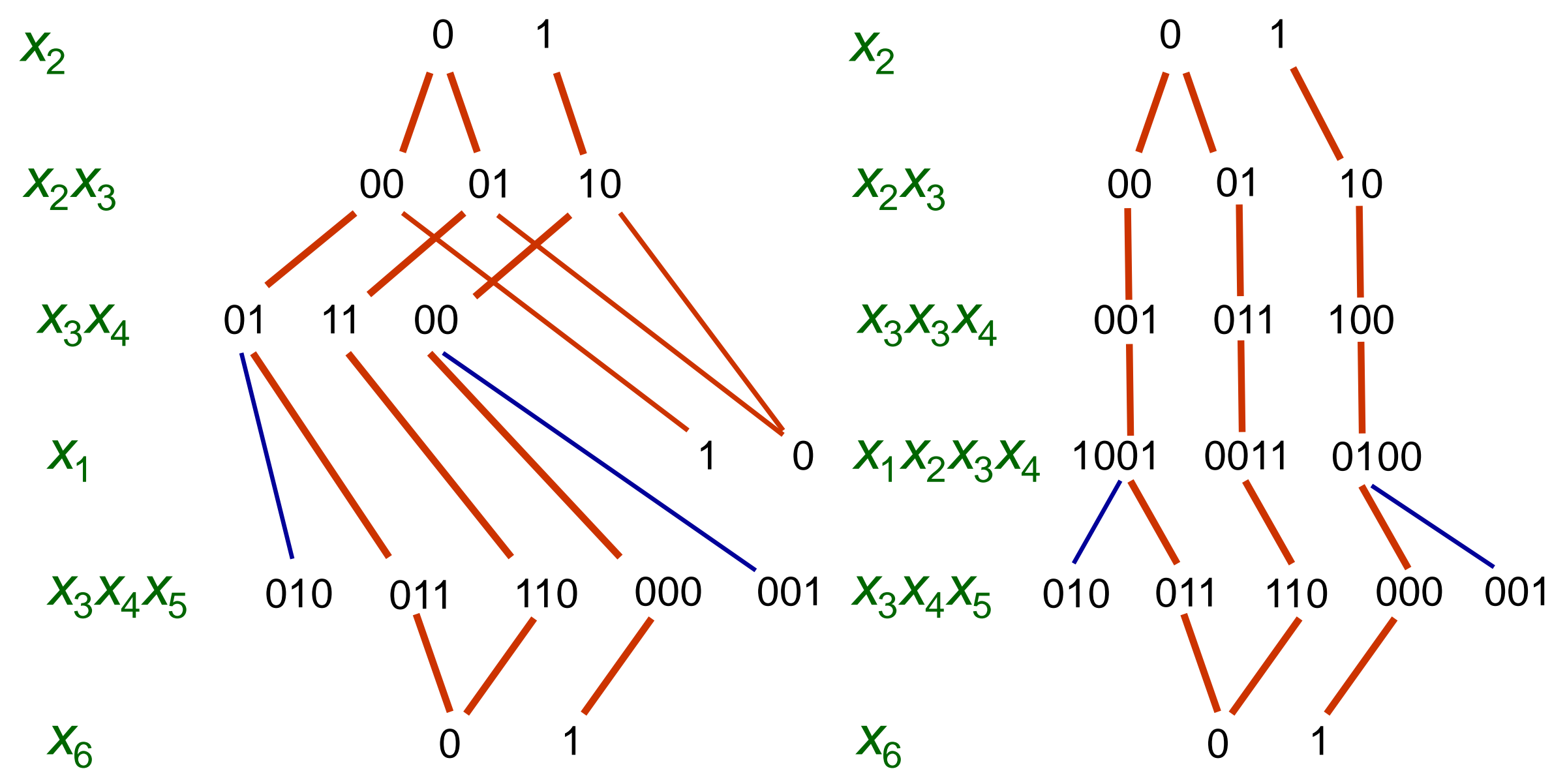

Feasible solutions 


\section{BDD vs. DP Solution}

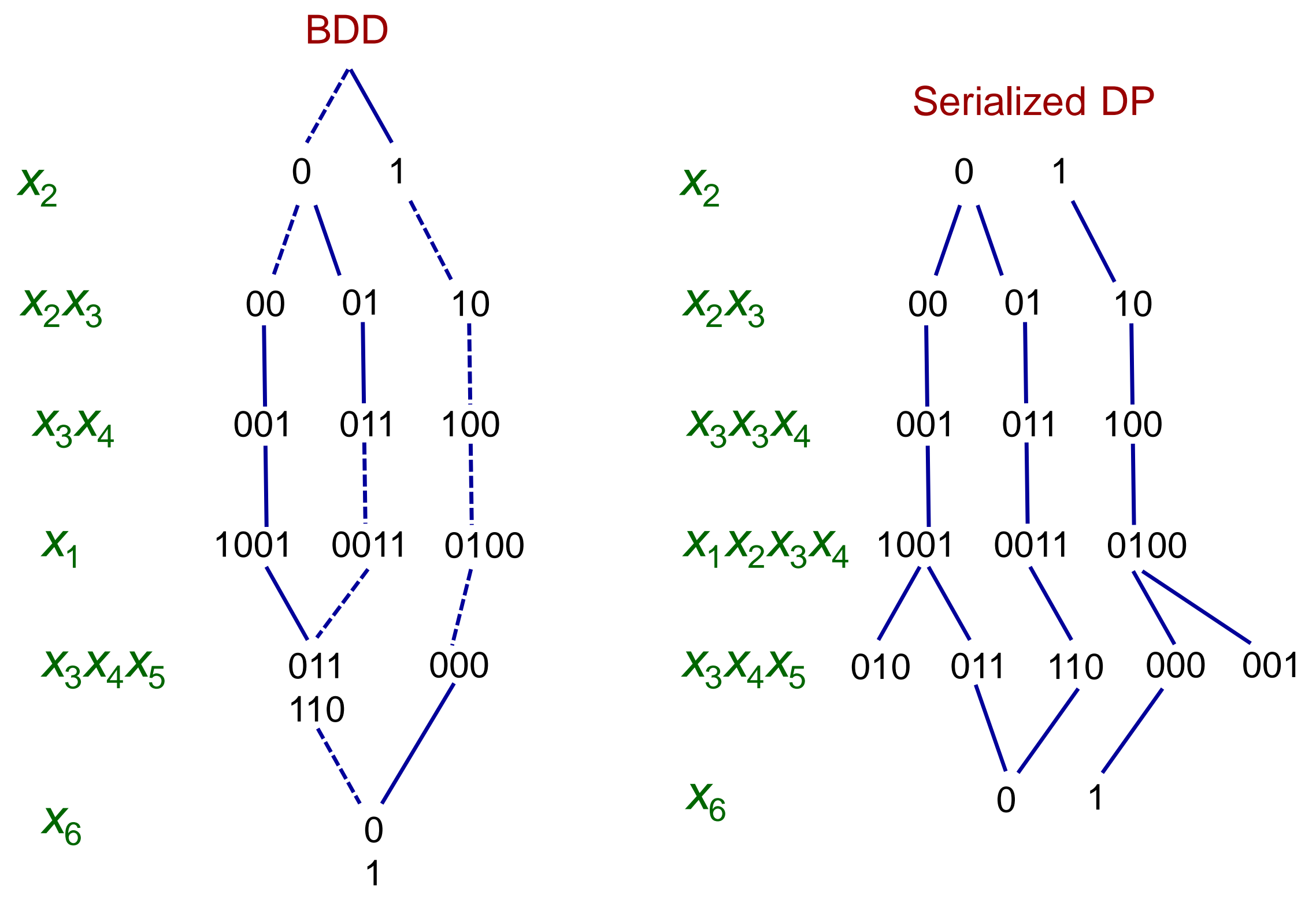




\section{BDD vs. DP Solution}

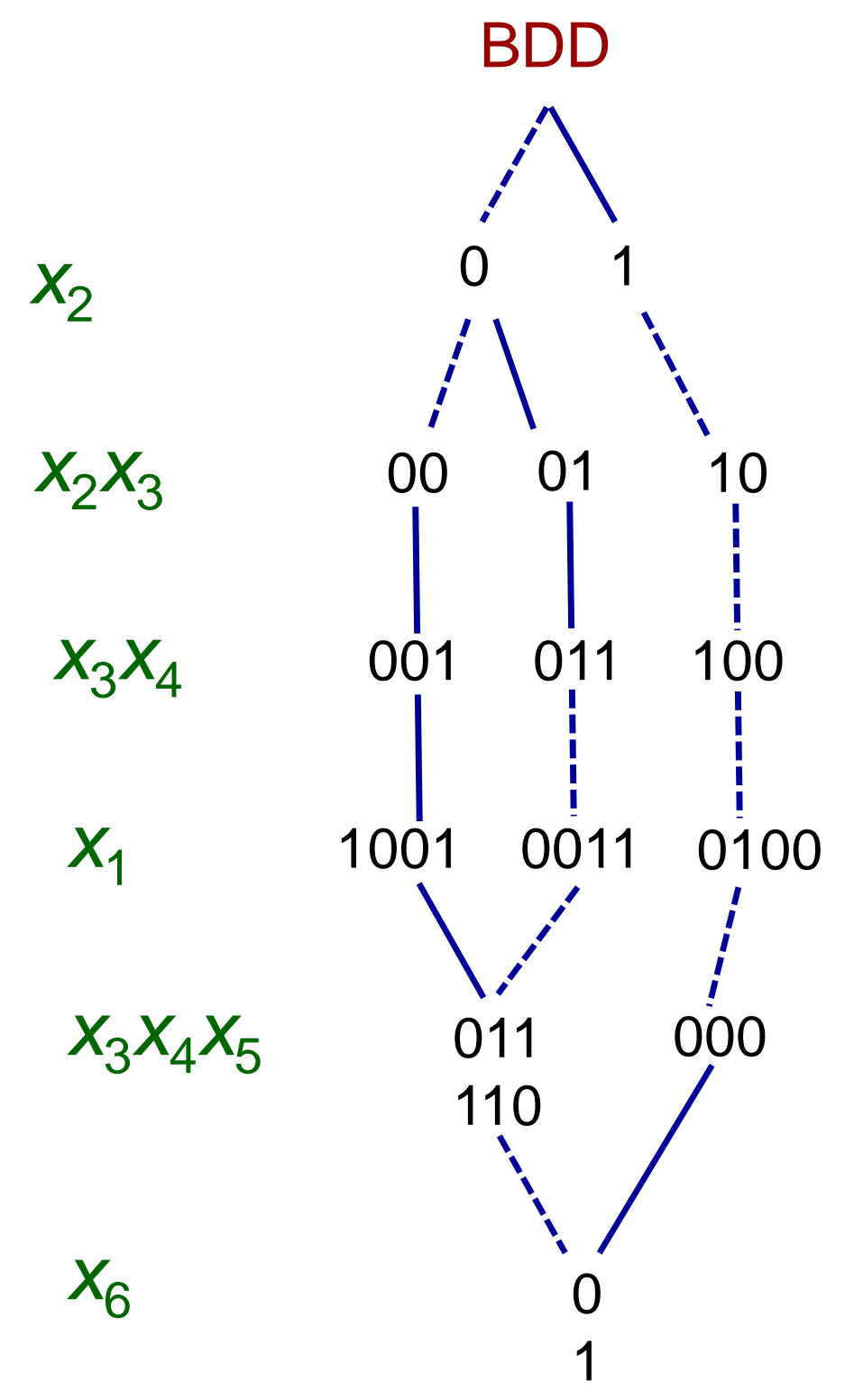

\section{Serialized DP}

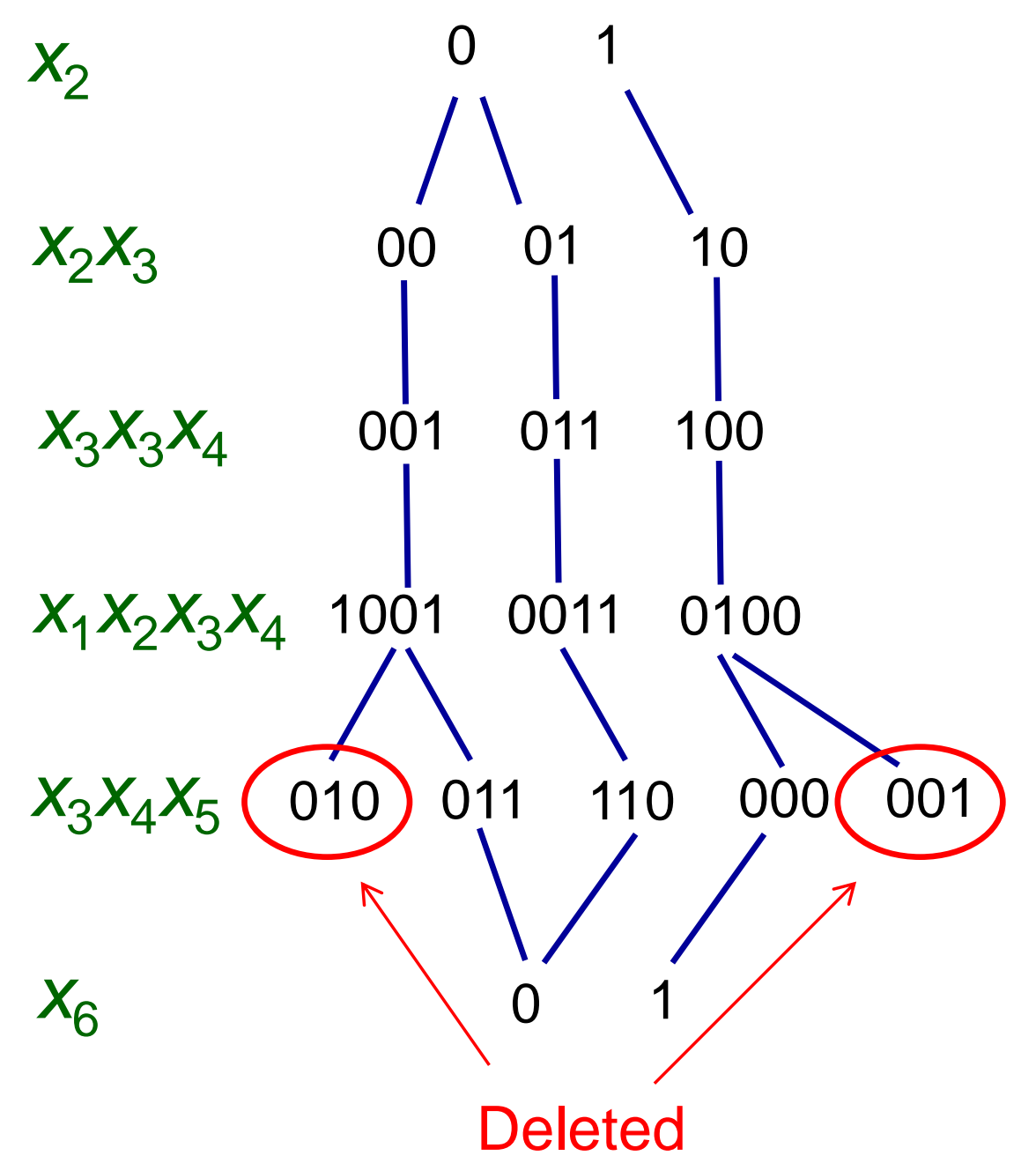




\section{BDD vs. DP Solution}

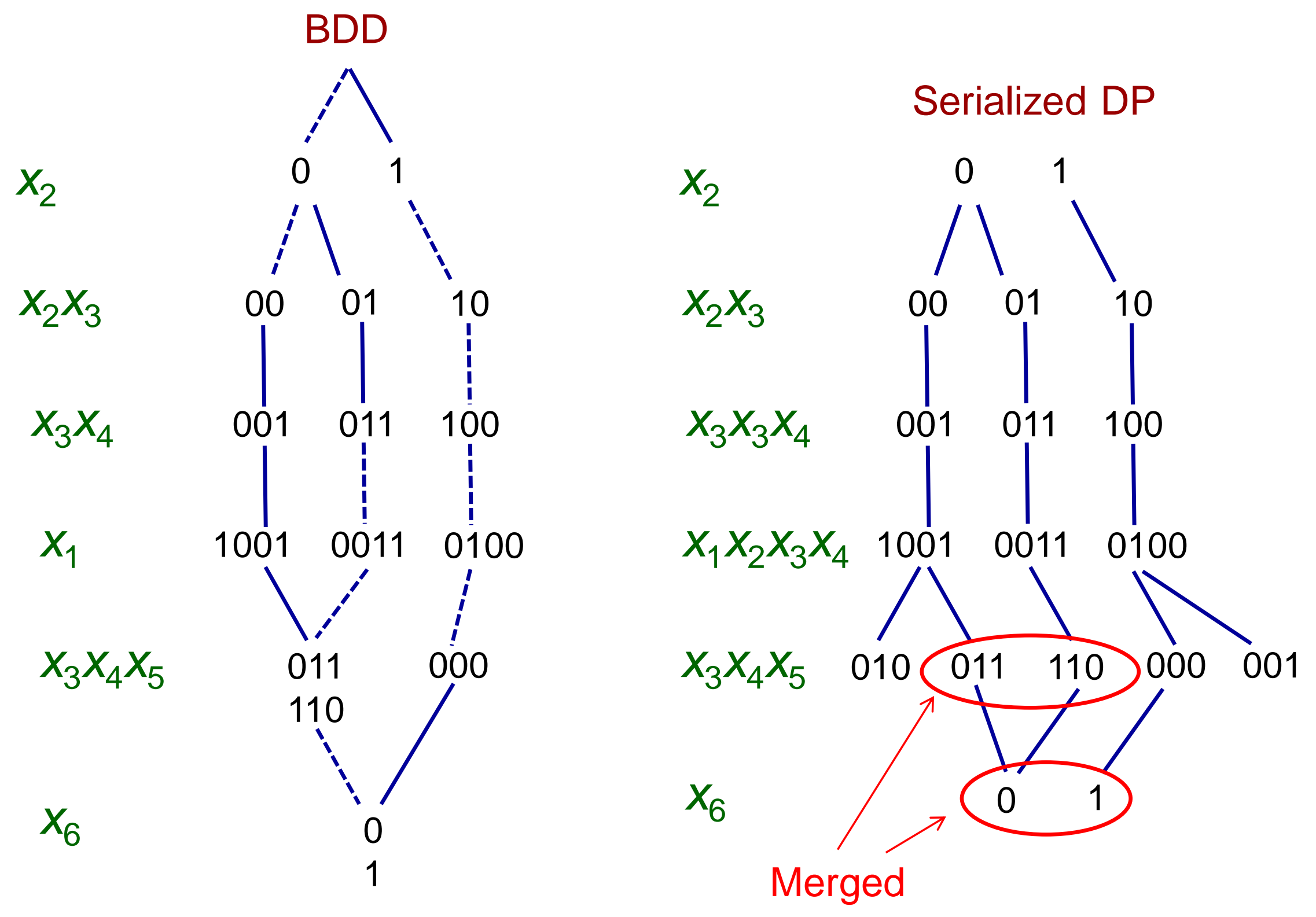




\section{Set Partitioning example}

Solution by nonserial DP

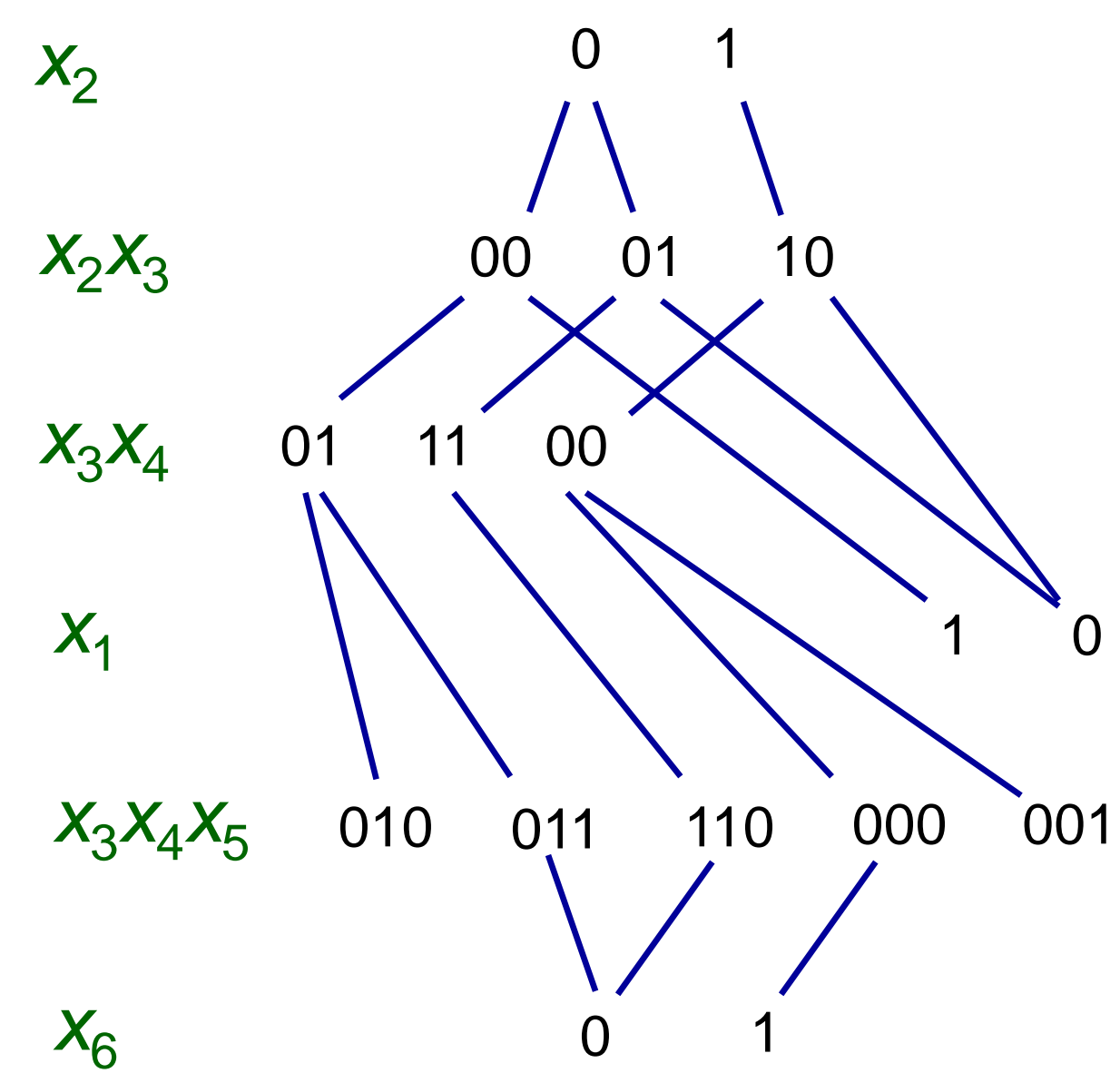




\section{Set Partitioning example}

\section{Nonserial BDD}

Solution by nonserial DP
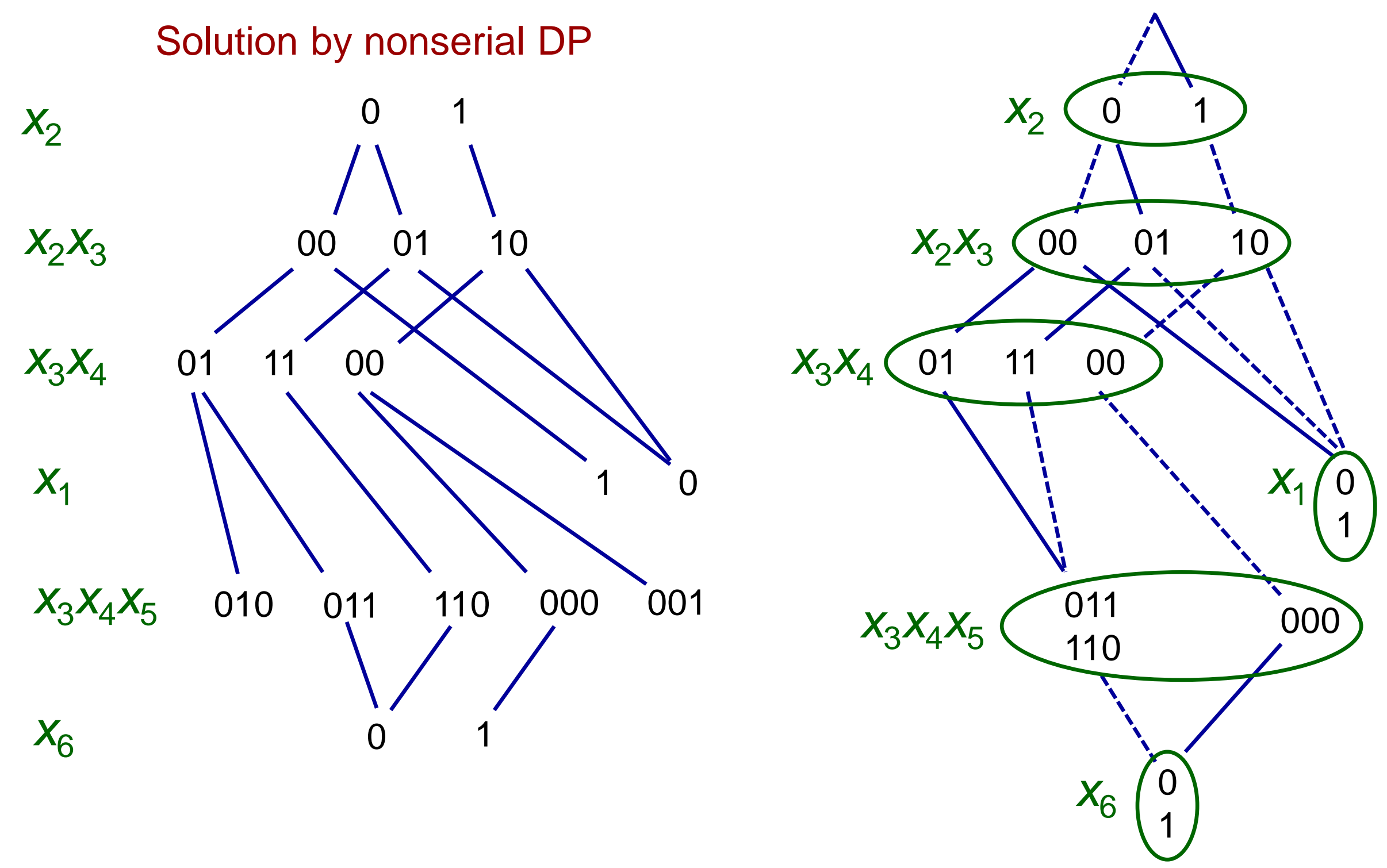


\section{Constructing the Join Tree}

$$
x_{2} x_{3} x_{4} x_{1} x_{5} x_{6}
$$

Clique in the dependency graph

$x_{1} x_{2} x_{3}$

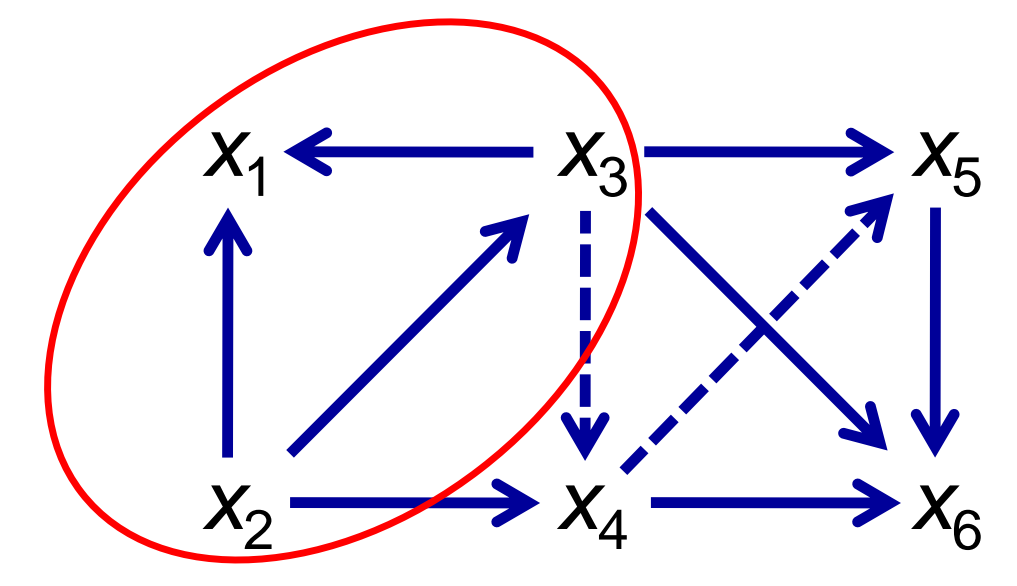




\section{Constructing the Join Tree}

$$
x_{2} x_{3} x_{4} x_{1} x_{5} x_{6}
$$

Clique in the dependency graph

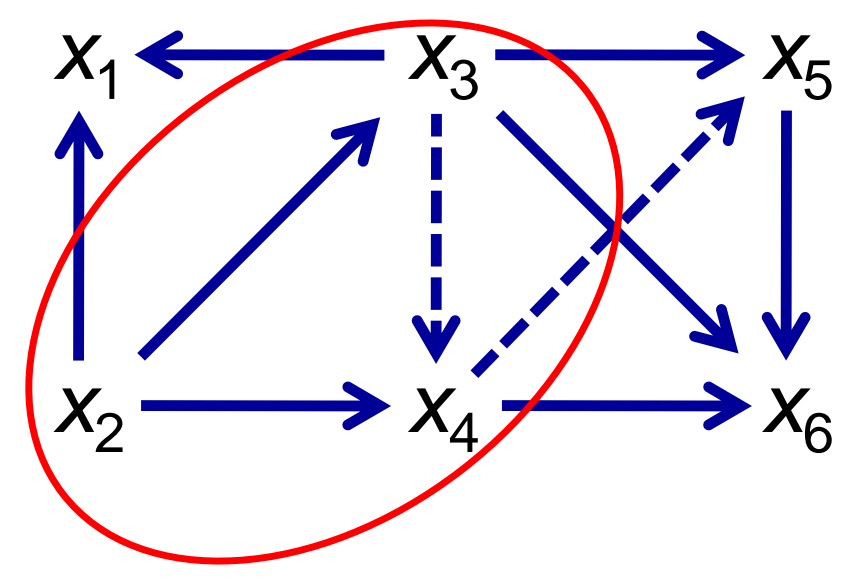

$x_{1} x_{2} x_{3}$

$x_{2} x_{3} x_{4}$ 


\section{Constructing the Join Tree}

$$
x_{2} x_{3} x_{4} x_{1} x_{5} x_{6}
$$

Clique in the dependency graph

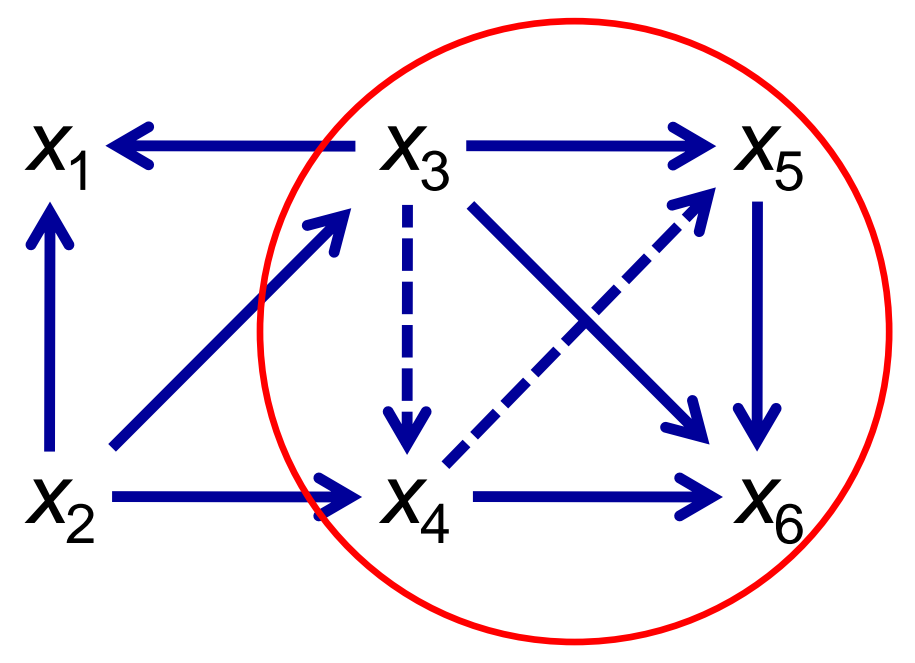

$x_{1} x_{2} x_{3}$

$x_{2} x_{3} x_{4}$

$x_{3} x_{4} x_{5} x_{6}$ 


\section{Constructing the Join Tree}

$$
x_{2} x_{3} x_{4} x_{1} x_{5} x_{6}
$$

Dependency graph
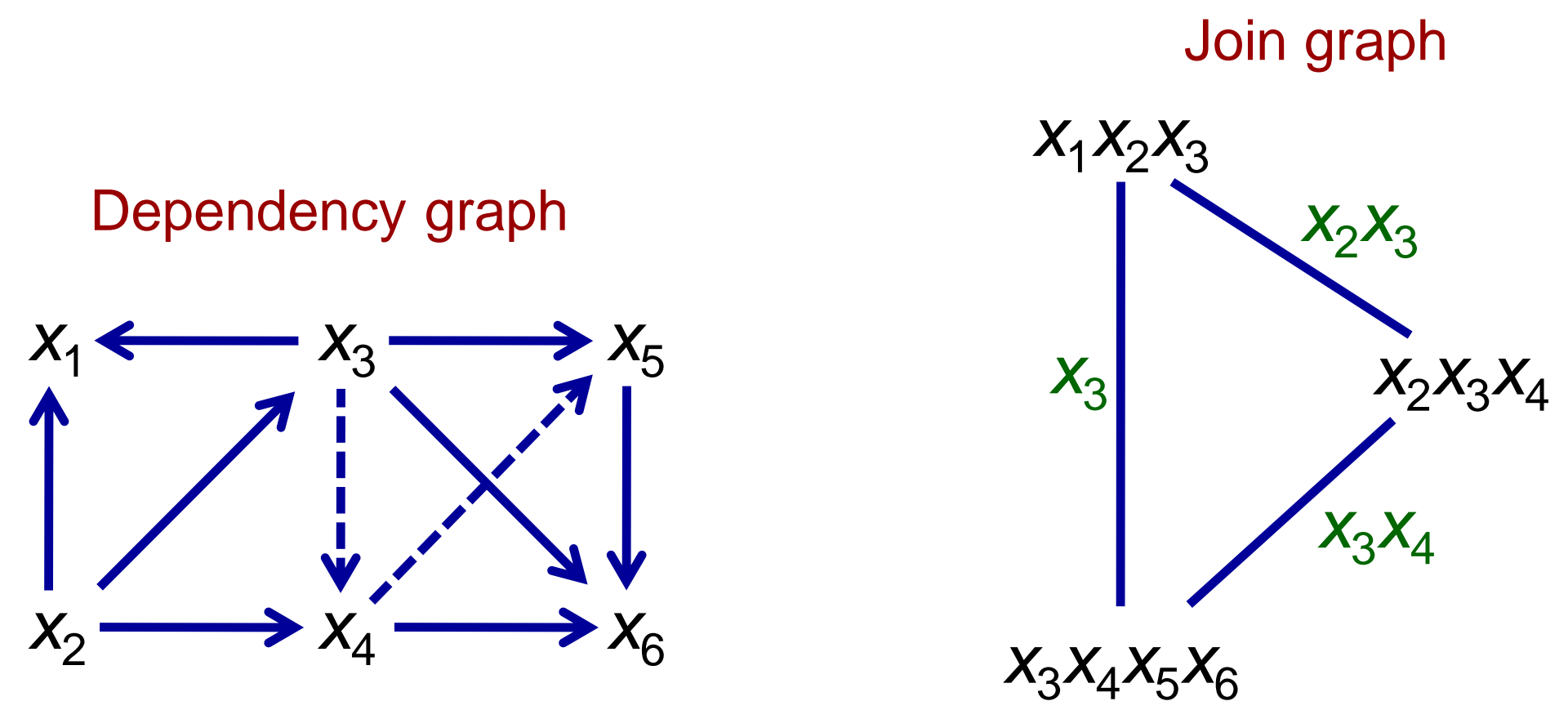

Connect nodes with common variables 


\section{Constructing the Join Tree}

$$
x_{2} x_{3} x_{4} x_{1} x_{5} x_{6}
$$

Dependency graph
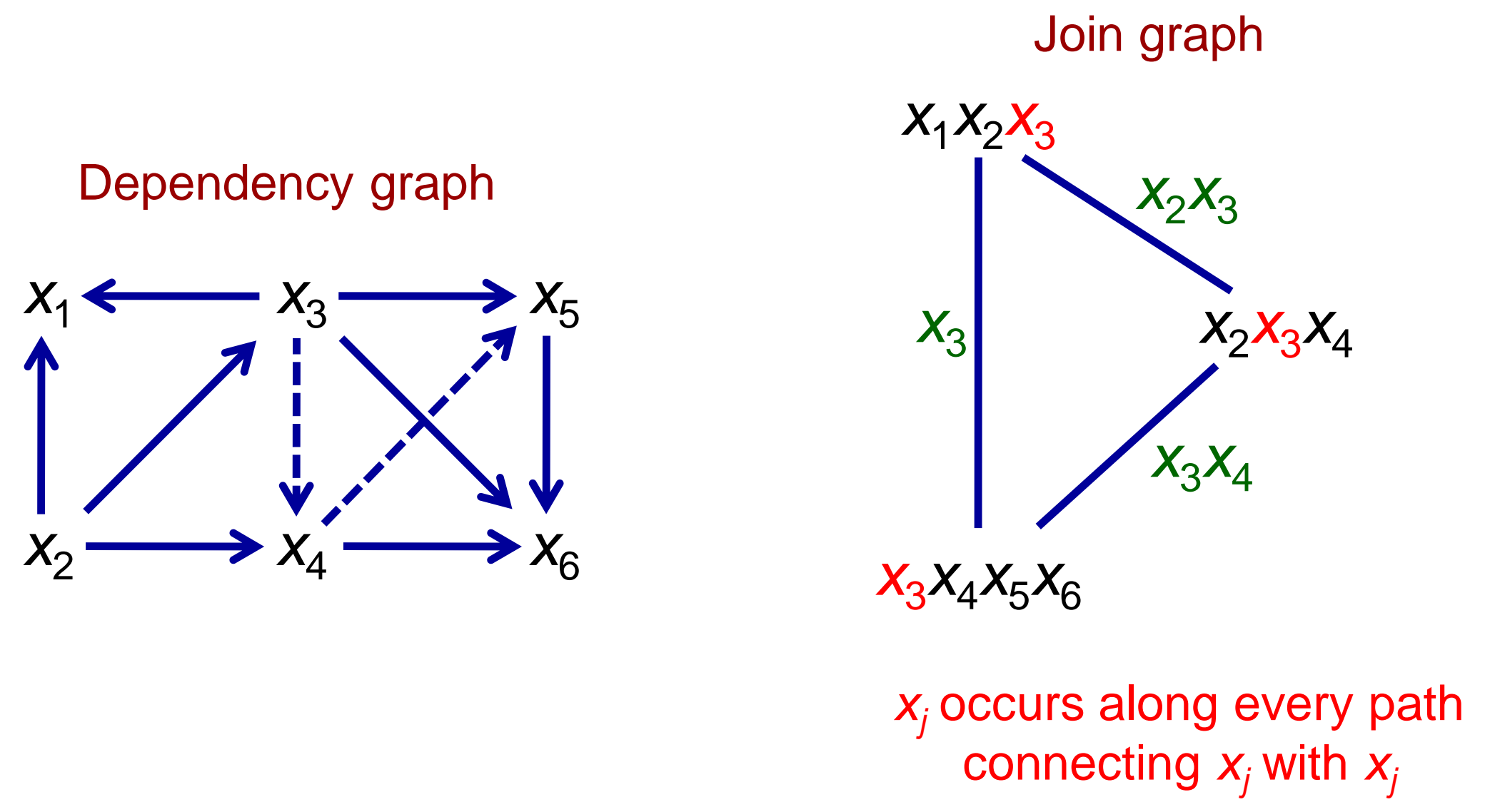


\section{Constructing the Join Tree}

$$
x_{2} x_{3} x_{4} x_{1} x_{5} x_{6}
$$

Dependency graph
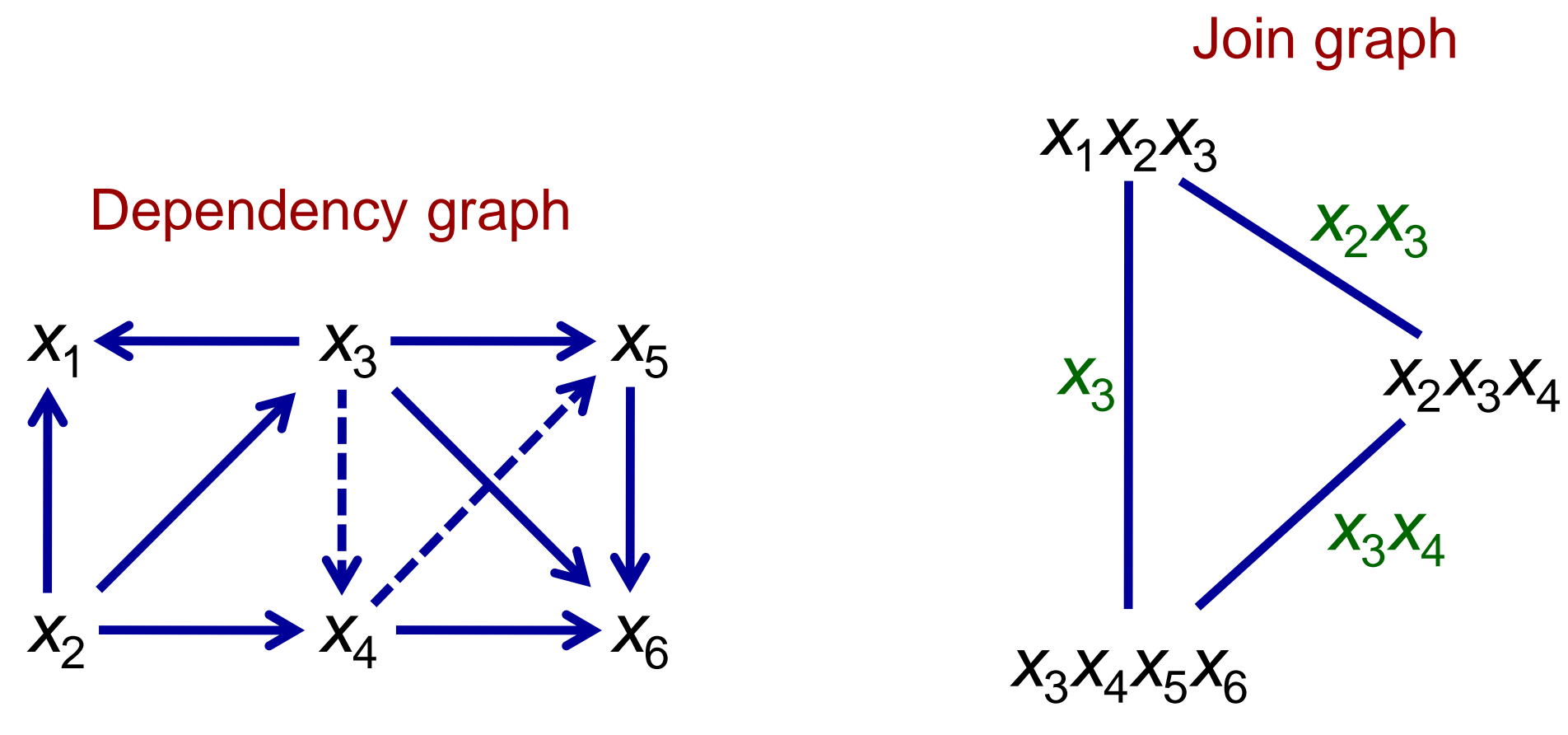

This can be viewed as the constraint dual

Binary constraints equate common variables in subproblems 


\section{Constructing the Join Tree}

$$
x_{2} x_{3} x_{4} x_{1} x_{5} x_{6}
$$

Dependency graph
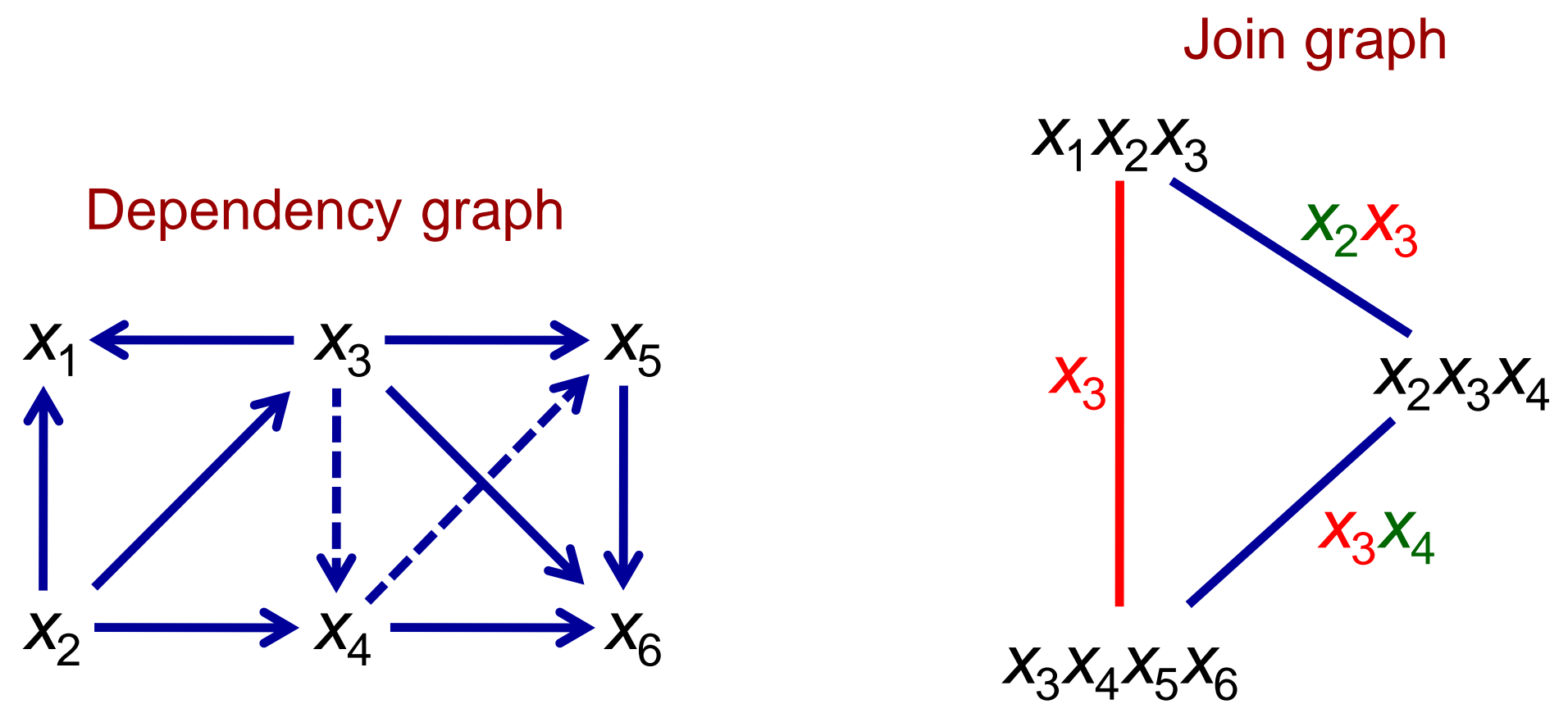

Some edges may be redundant when equating variables 


\section{Constructing the Join Tree}

$$
x_{2} x_{3} x_{4} x_{1} x_{5} x_{6}
$$

Dependency graph
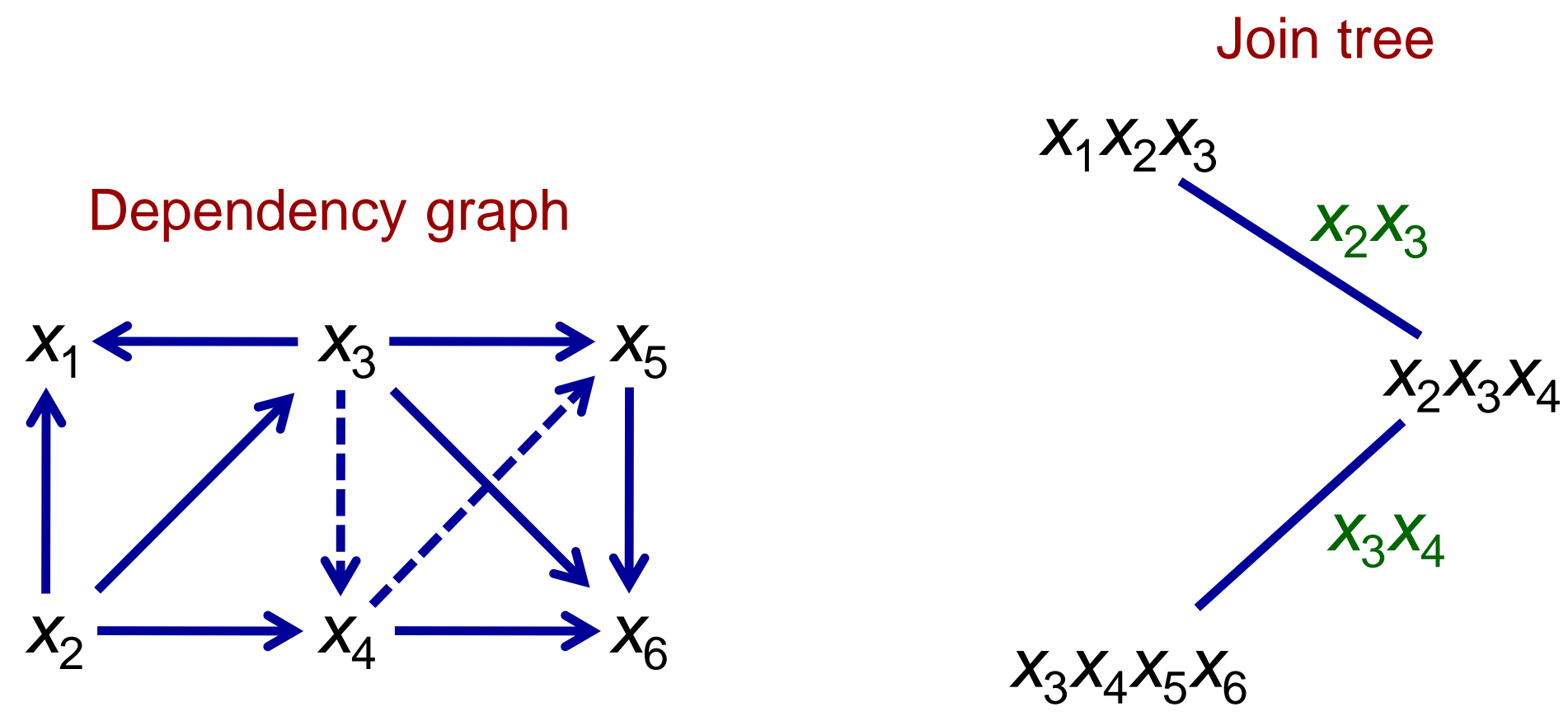

Removing redundant edges yields join tree 


\section{Constructing the Join Tree}

$$
x_{2} x_{3} x_{4} x_{1} x_{5} x_{6}
$$

Dependency graph

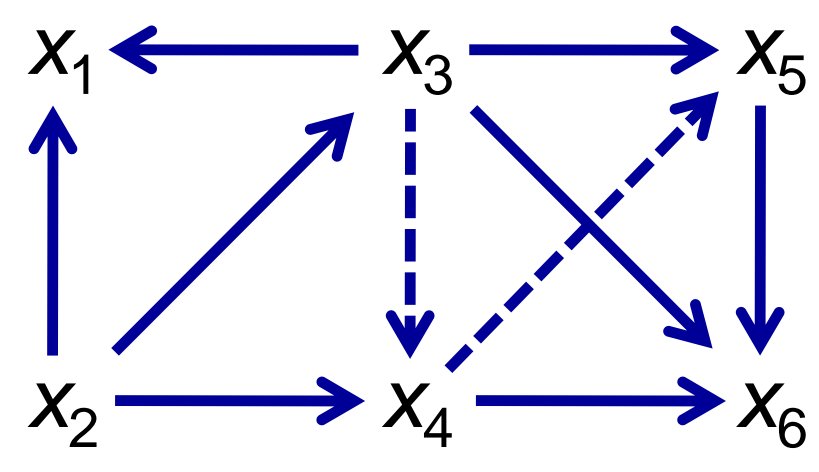

Join tree

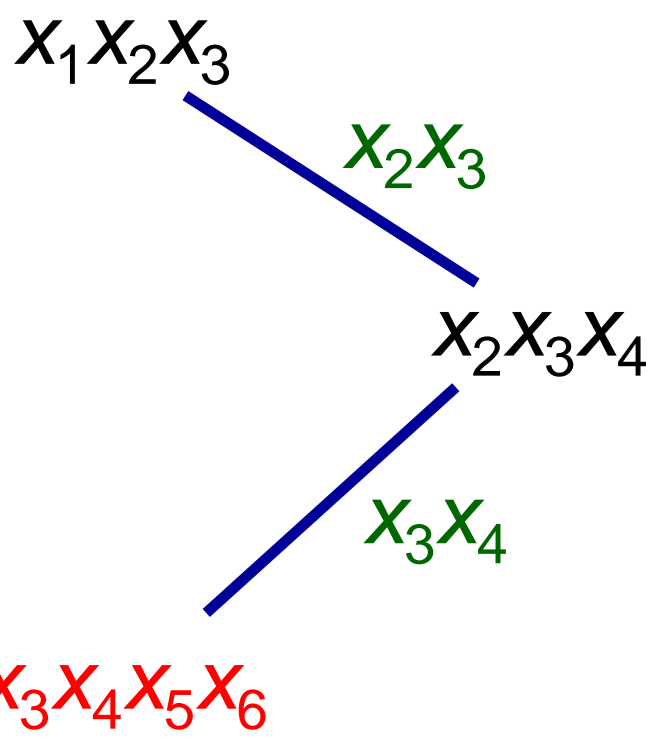

Max node cardinality is tree width $+1=3+1$ 


\title{
Constructing the Join Tree
}

$$
x_{2} x_{3} x_{4} x_{1} x_{5} x_{6}
$$

Dependency graph

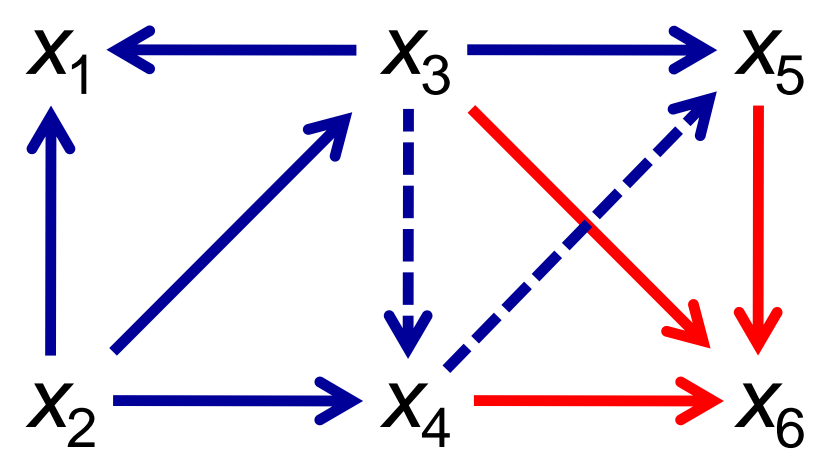

\author{
Join tree
}

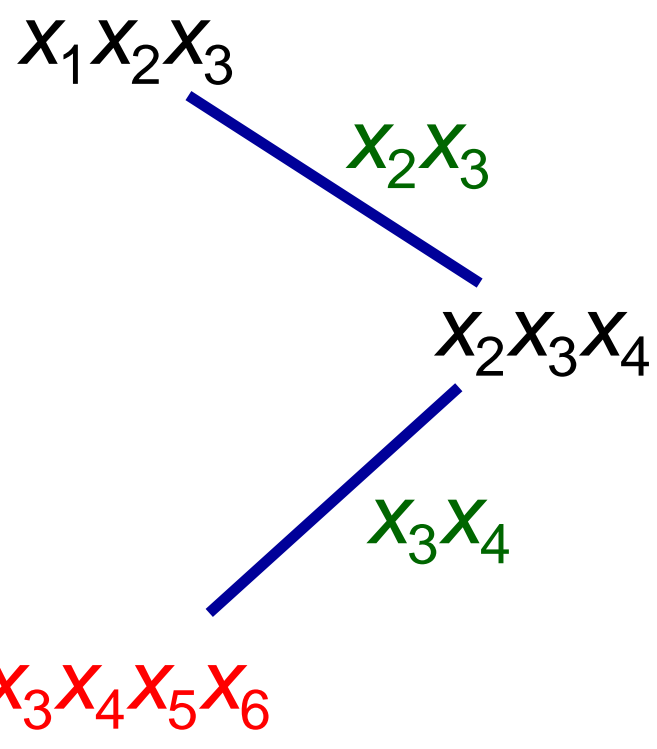

Induced width $=$ tree width $=3$ 


\section{Designing the Nonserial BDD}

$$
x_{2} x_{3} x_{4} x_{1} x_{5} x_{6}
$$

BDD design

$x_{2}$
Join tree

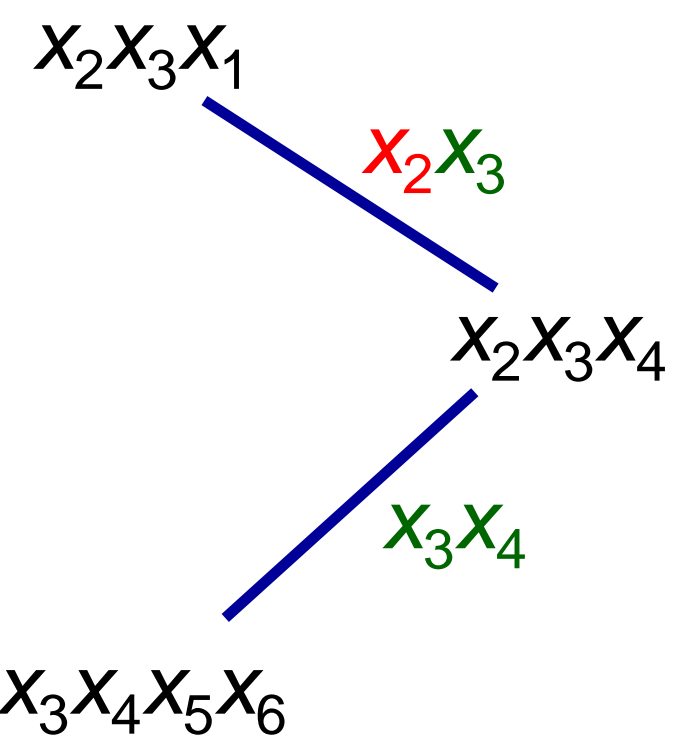




\section{Designing the Nonserial BDD}

$$
x_{2} x_{3} x_{4} x_{1} x_{5} x_{6}
$$

BDD design

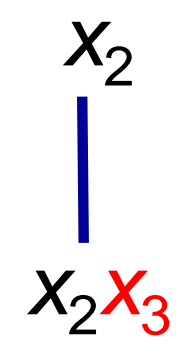

\author{
Join tree
}

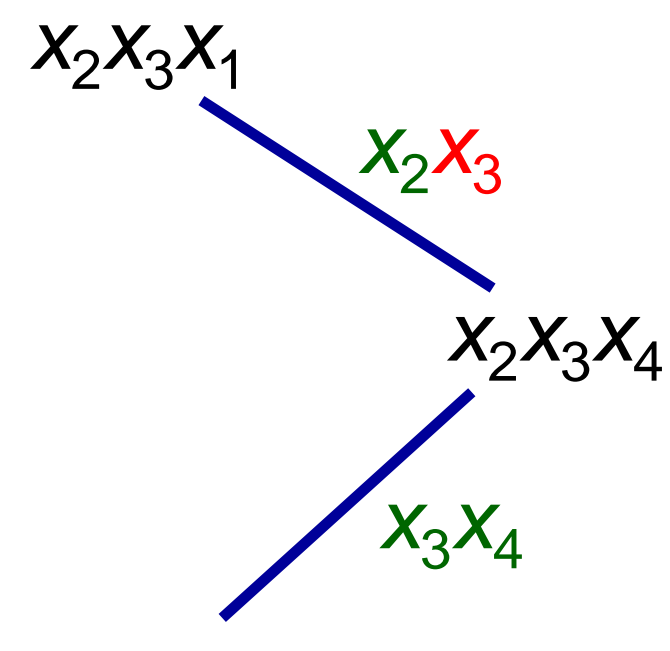

$x_{3} x_{4} x_{5} x_{6}$ 


\section{Designing the Nonserial BDD}

$$
x_{2} x_{3} x_{4} x_{1} x_{5} x_{6}
$$

\section{BDD design}

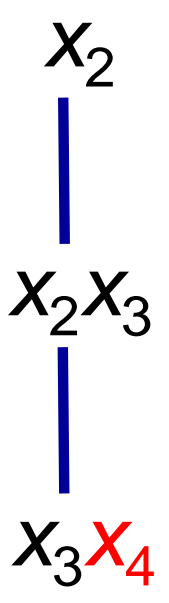

\author{
Join tree
}

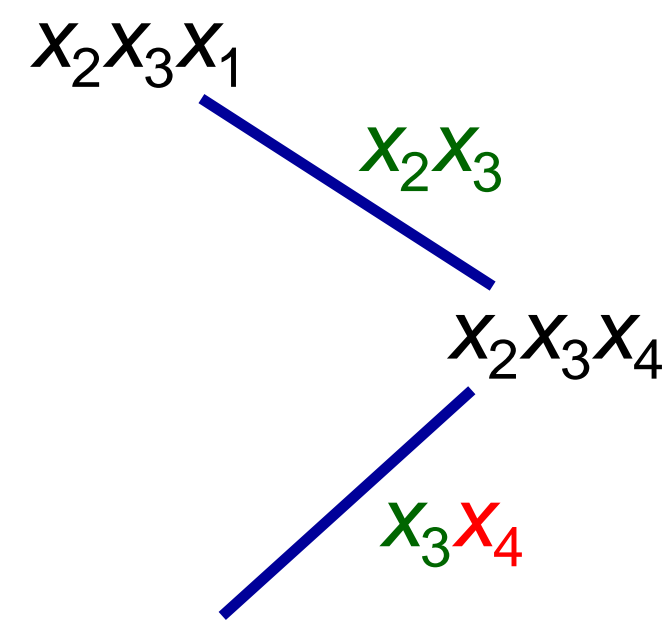

$x_{3} x_{4} x_{5} x_{6}$ 


\section{Designing the Nonserial BDD}

$$
x_{2} x_{3} x_{4} x_{1} x_{5} x_{6}
$$

\section{BDD design}

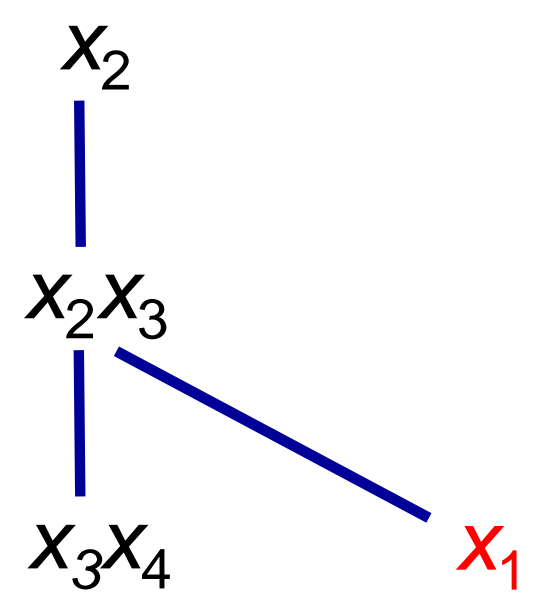

\author{
Join tree
}

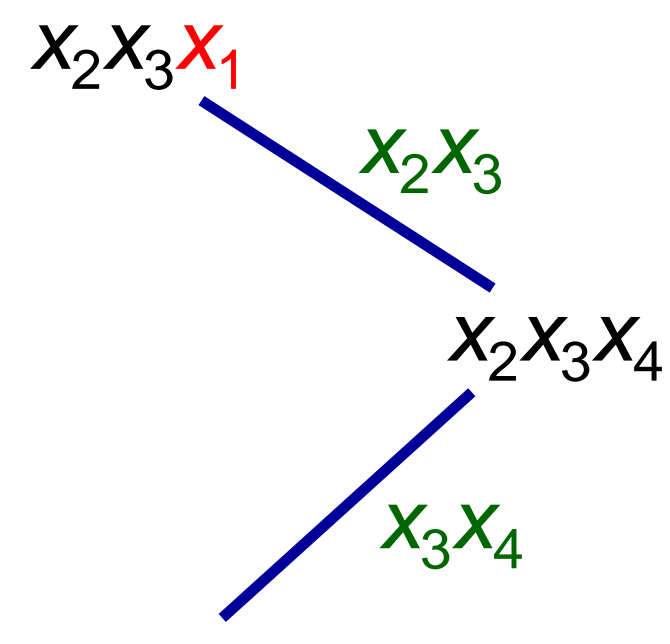

$x_{3} x_{4} x_{5} x_{6}$ 


\section{Designing the Nonserial BDD}

$$
x_{2} x_{3} x_{4} x_{1} x_{5} x_{6}
$$

\section{BDD design}

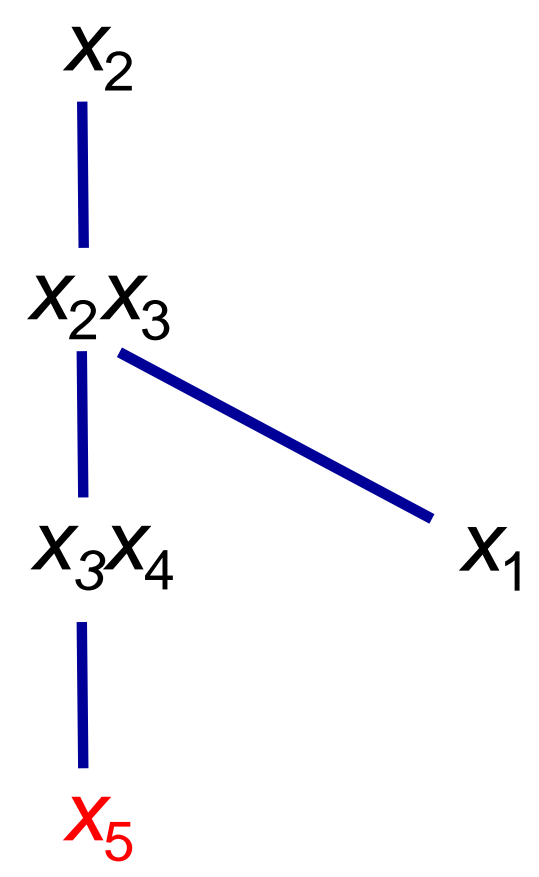

\author{
Join tree
}

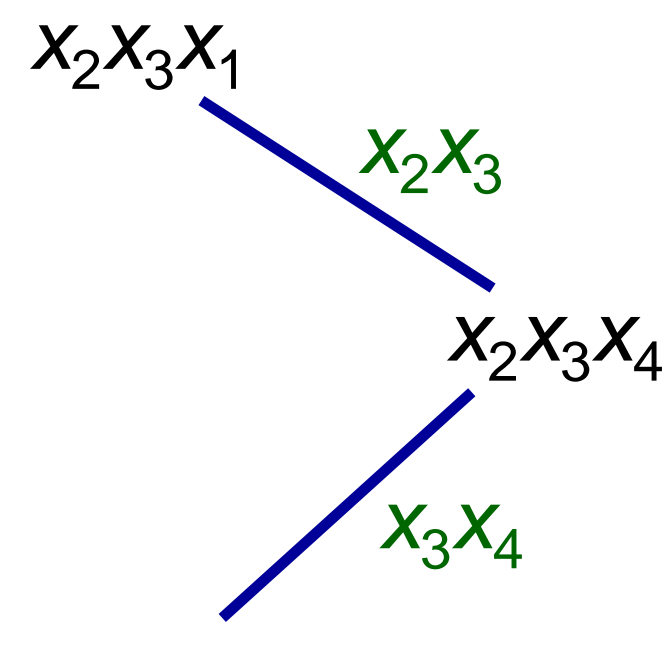

$x_{3} x_{4} x_{5} x_{6}$ 


\section{Designing the Nonserial BDD}

$$
x_{2} x_{3} x_{4} x_{1} x_{5} x_{6}
$$

BDD design

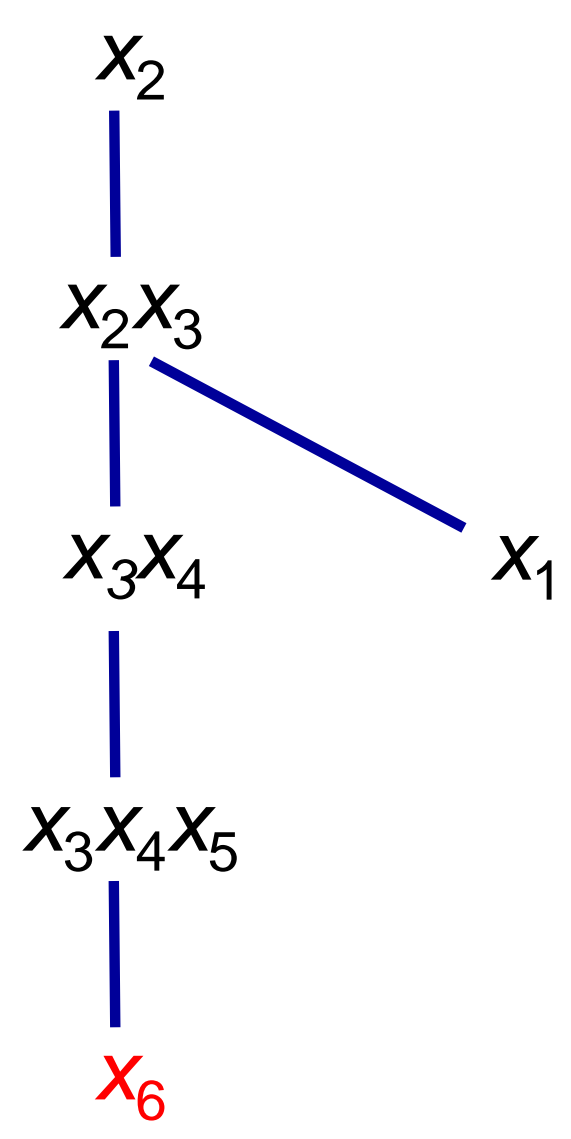

Join tree

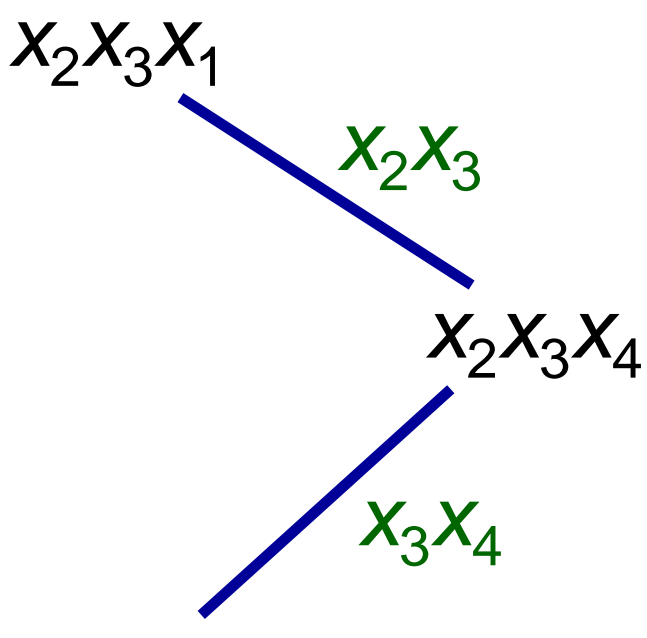

$x_{3} x_{4} x_{5} x_{6}$ 


\section{Designing the Nonserial BDD}

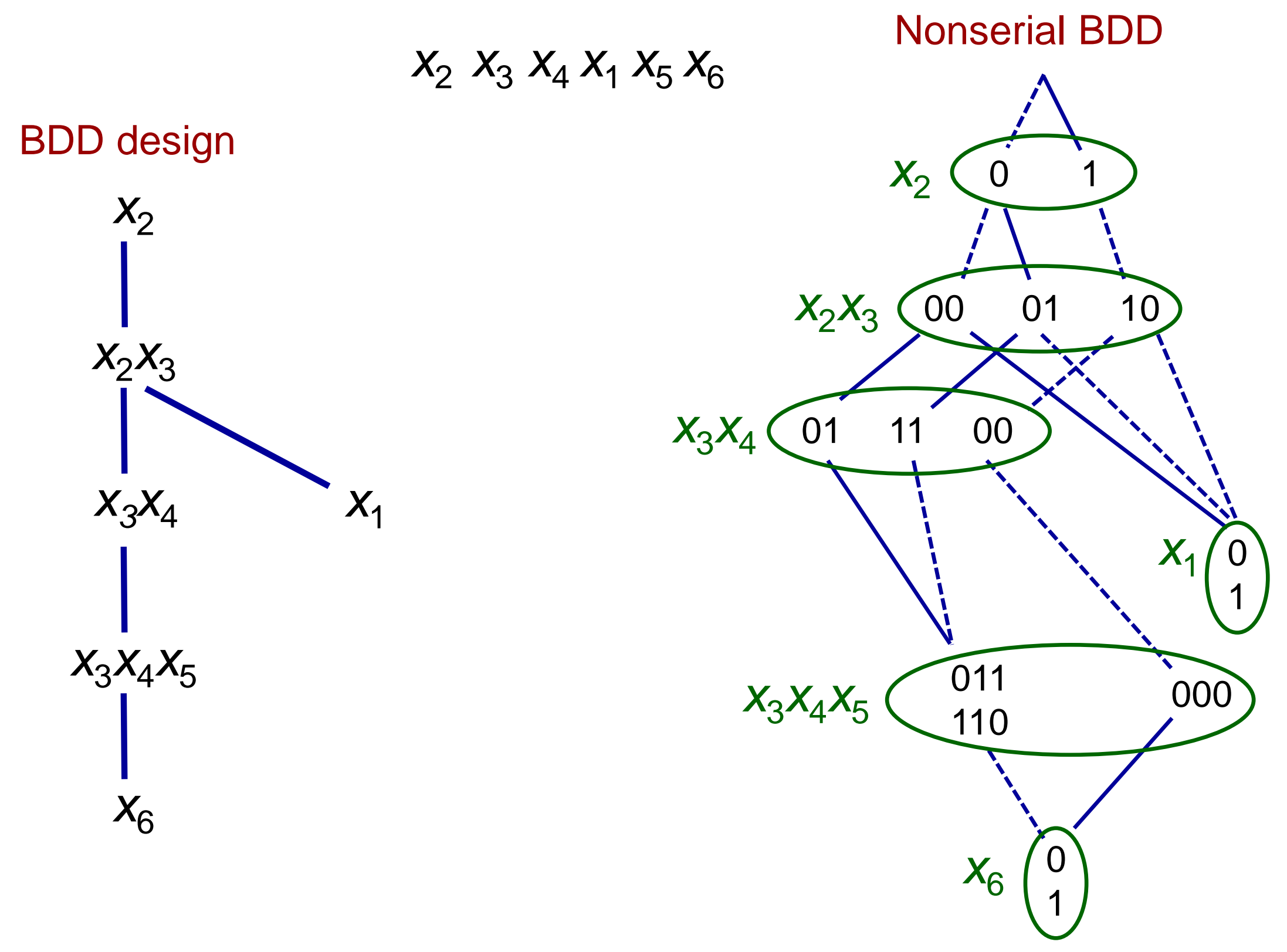




\section{Another Variable Ordering}

$$
x_{3} x_{6} x_{2} x_{5} x_{1} x_{4}
$$

Dependency graph
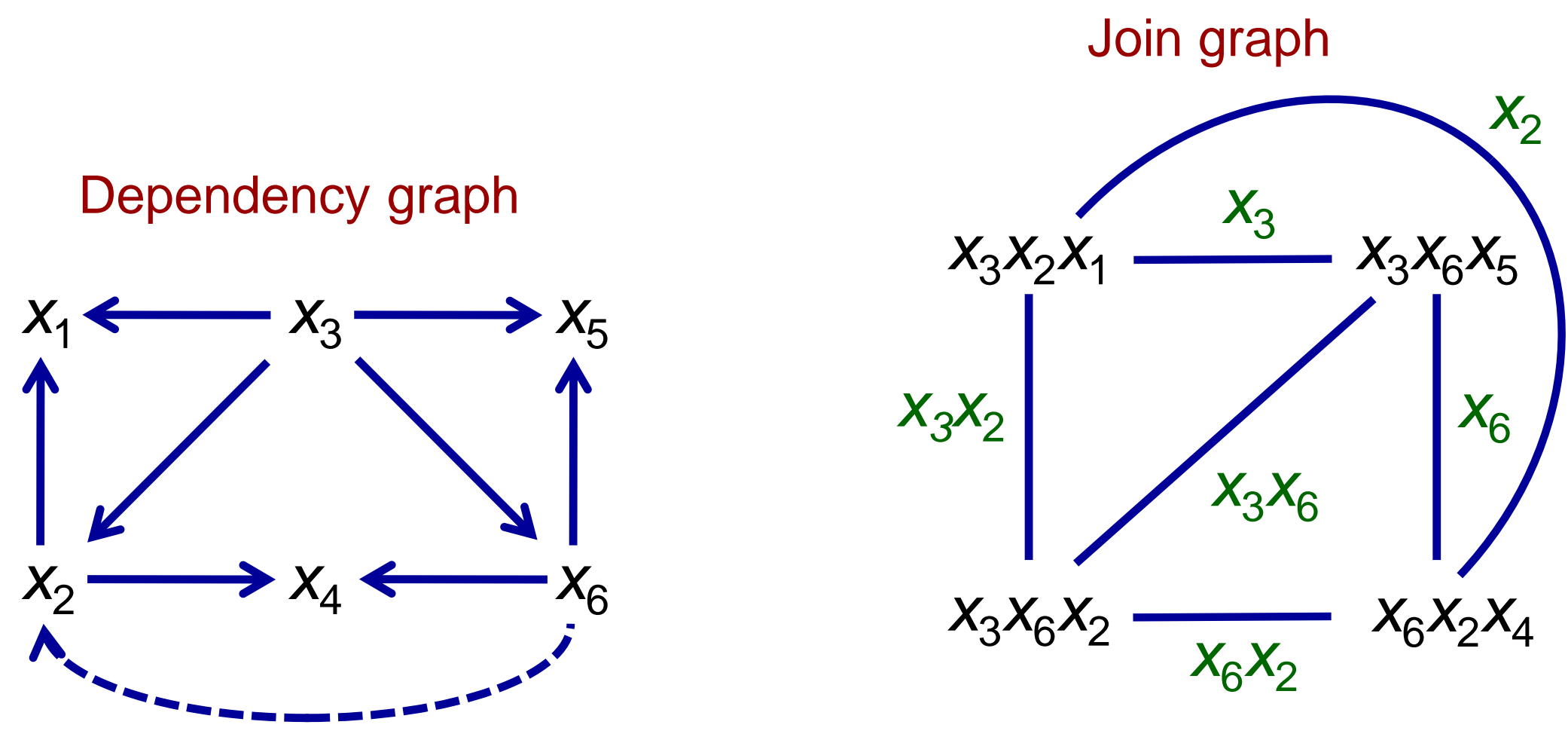

Induced width $=2$ 


\section{Constructing the Join Tree}

$$
x_{3} x_{6} x_{2} x_{5} x_{1} x_{4}
$$

Dependency graph

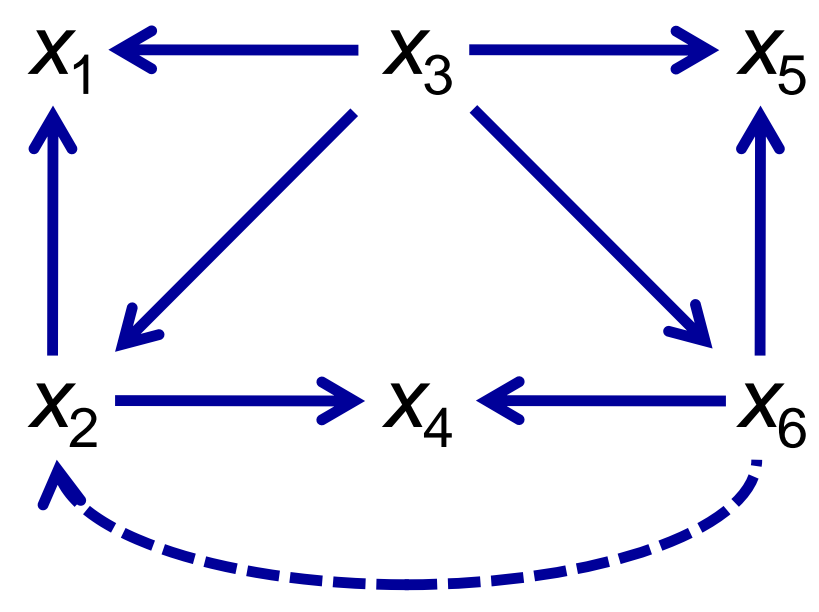

Induced width $=2$

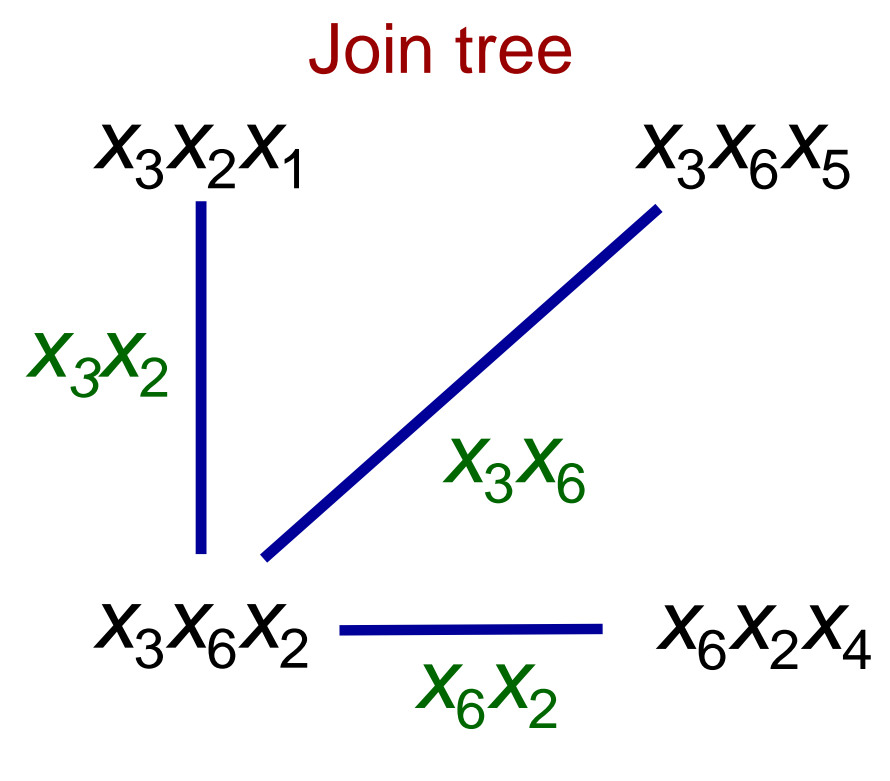

Tree width $=2$ 


\section{Designing the BDD}

$$
x_{3} x_{6} x_{2} x_{5} x_{1} x_{4}
$$

\section{BDD design}

$x_{3}$

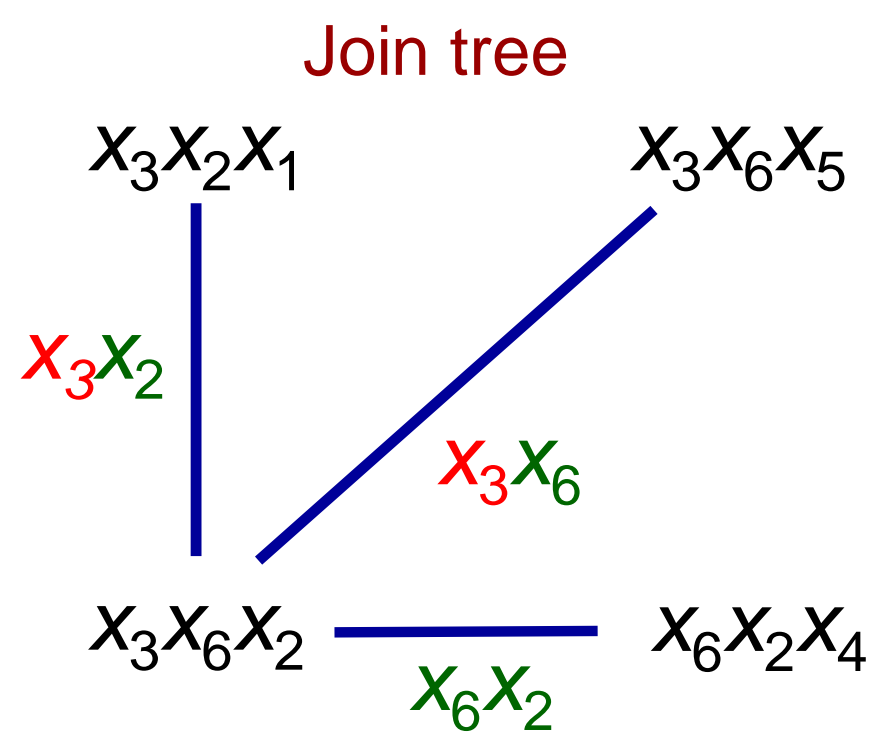

Tree width $=2$ 


\section{Designing the BDD}

$$
x_{3} x_{6} x_{2} x_{5} x_{1} x_{4}
$$

\section{BDD design}
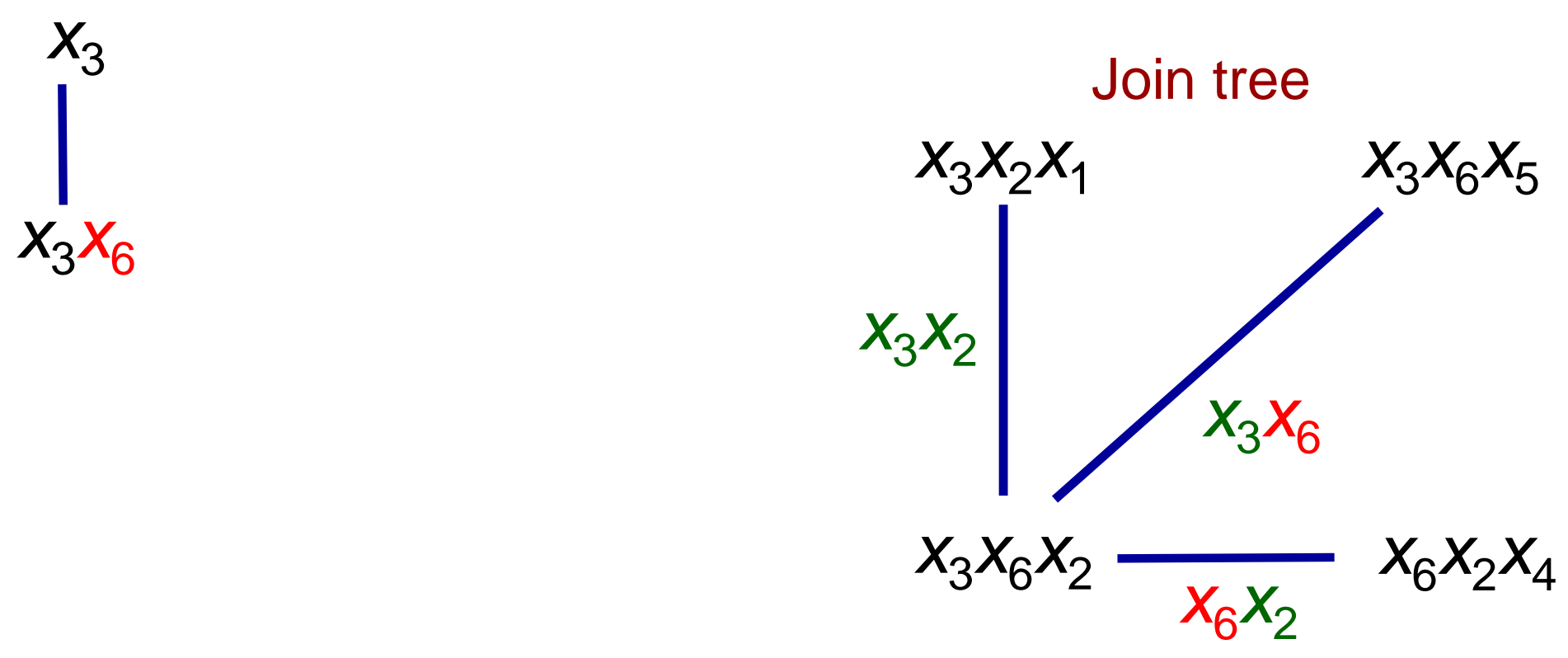

Tree width $=2$ 


\section{Designing the BDD}

$$
x_{3} x_{6} x_{2} x_{5} x_{1} x_{4}
$$

\section{BDD design}
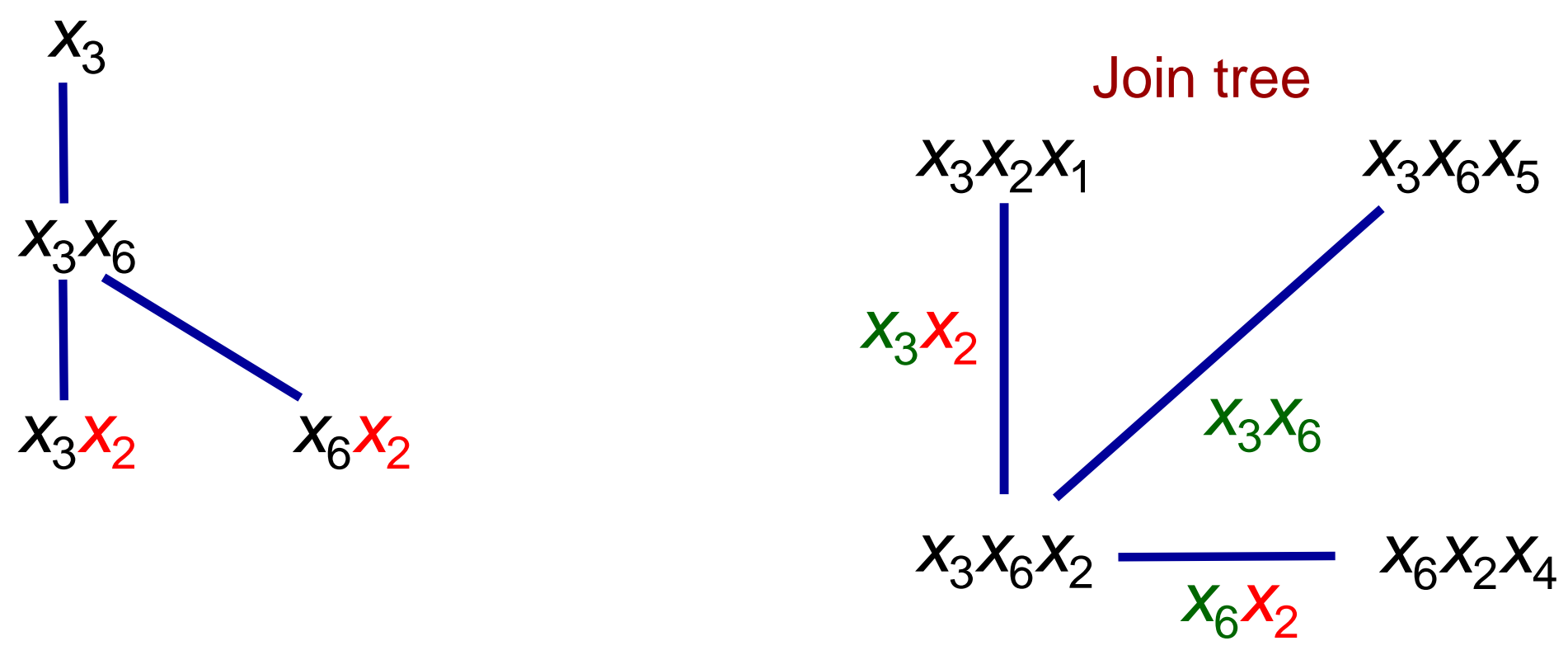

Tree width $=2$ 


\section{Designing the BDD}

$$
x_{3} x_{6} x_{2} x_{5} x_{1} x_{4}
$$

\section{BDD design}
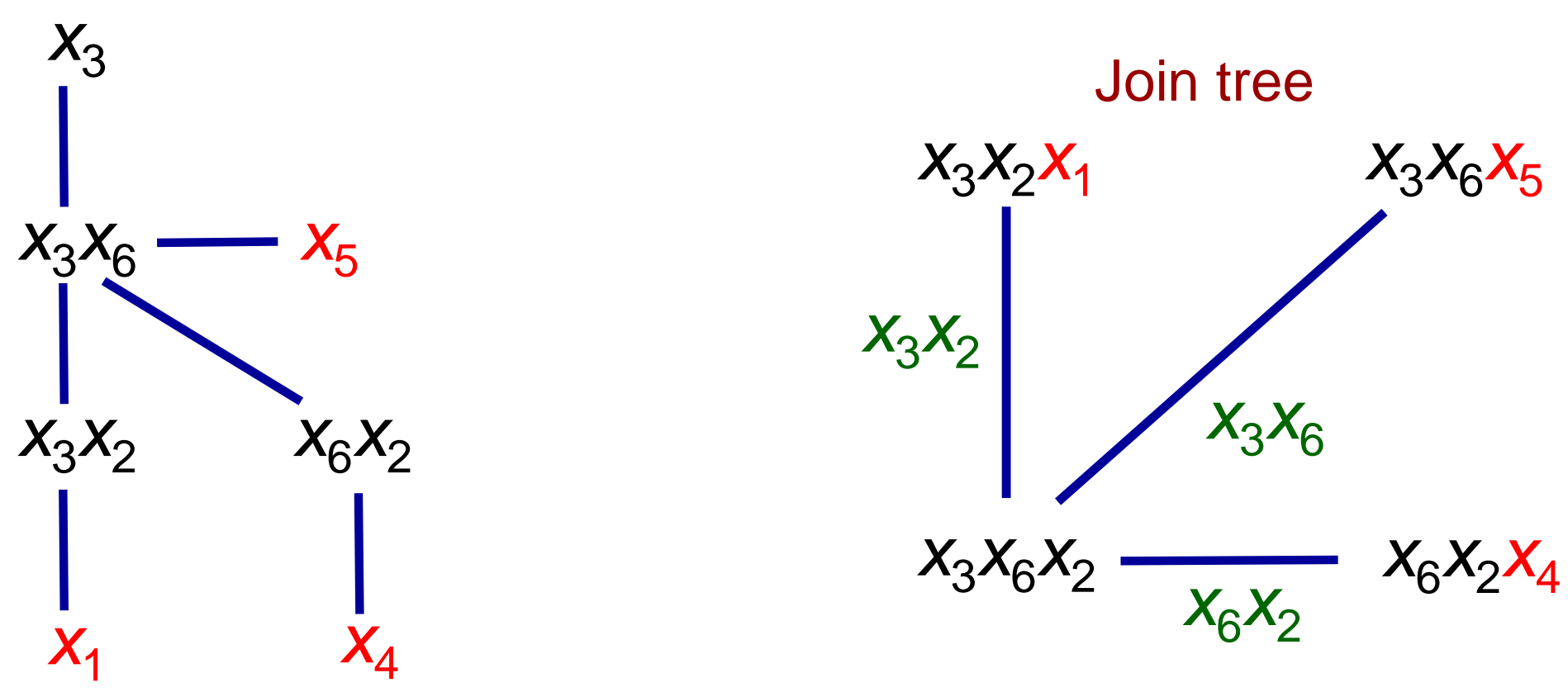

Tree width $=2$ 


\section{Nonserial BDD}

\section{$x_{3} x_{6} x_{2} x_{5} x_{1} x_{4} \quad$ Nonserial BDD}

BDD design

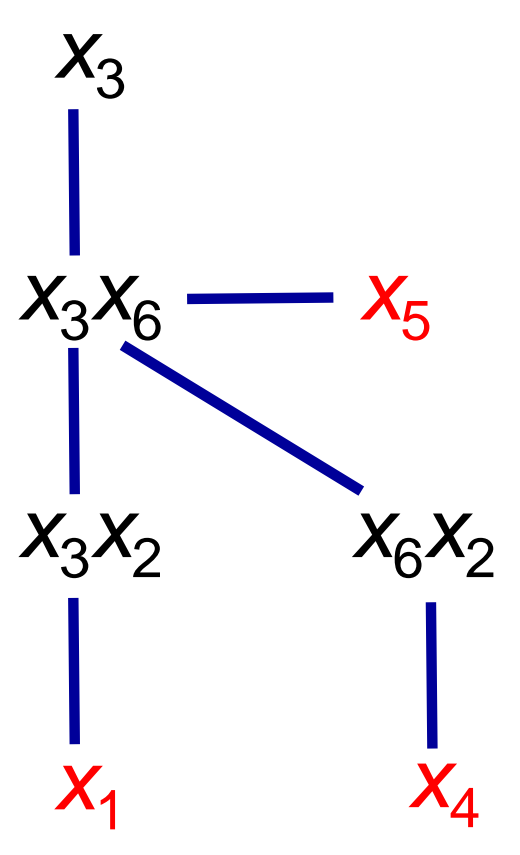

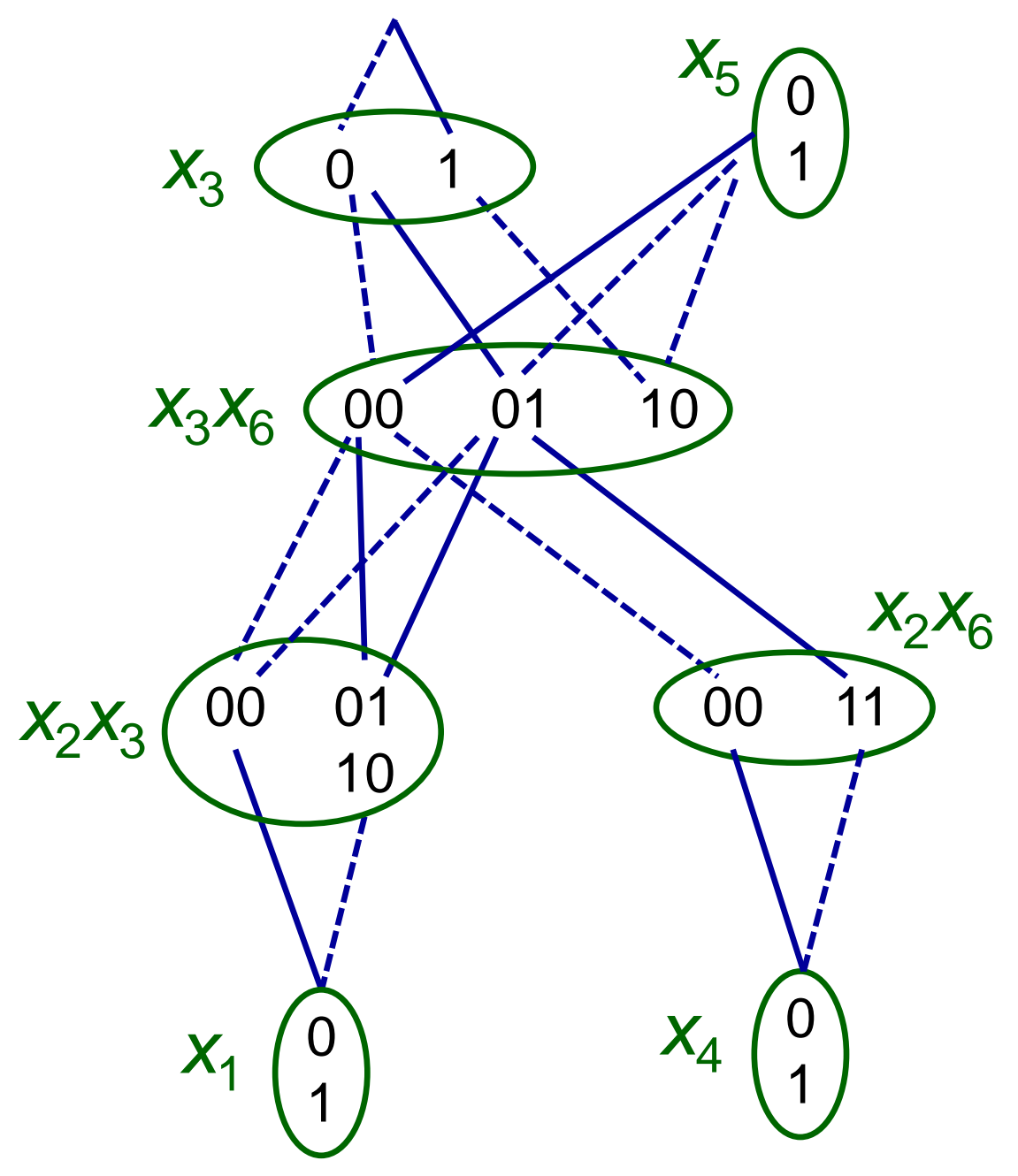




\section{Current Research}

- Broader applicability

- Stochastic dynamic programming

- Continuous global optimization

- Combination with other techniques

- Lagrangean relaxation.

- Column generation

- Logic-based Benders decomposition

- Solve separation problem 


\section{References}

2006

- T. Hadzic and J. N. Hooker. Discrete global optimization with binary decision diagrams. In Workshop on Global Optimization: Integrating Convexity, Optimization, Logic Programming, and Computational Algebraic Geometry (GICOLAG), Vienna, 2006.

\section{7}

- Tarik Hadzic and J. N. Hooker. Cost-bounded binary decision diagrams for 0-1 programming. In Proceedings of CPAIOR. LNCS 4510, pp. 84-98. Springer, 2007.

- Tarik Hadzic and J. N. Hooker. Postoptimality analysis for integer programming using binary decision diagrams. December 2007, revised April 2008 (not submitted).

- M. Behle. Binary Decision Diagrams and Integer Programming. PhD thesis, Max Planck Institute for Computer Science, 2007.

- H. R. Andersen, T. Hadzic, J. N. Hooker, and P. Tiedemann. A constraint store based on multivalued decision diagrams. In Proceedings of CP. LNCS 4741, pp. 118-132. Springer, 2007.

\section{8}

- T. Hadzic, J. N. Hooker, B. O'Sullivan, and P. Tiedemann. Approximate compilation of constraints into multivalued decision diagrams. In Proceedings of CP. LNCS 5202, pp. 448462. Springer, 2008.

- T. Hadzic, J. N. Hooker, and P. Tiedemann. Propagating separable equalities in an MDD store. In Proceedings of CPAIOR. LNCS 5015, pp. 318-322. Springer, 2008. 


\section{References}

2010

- S. Hoda. Essays on Equilibrium Computation, MDD-based Constraint Programming and Scheduling. PhD thesis, Carnegie Mellon University, 2010.

- S. Hoda, W.-J. van Hoeve, and J. N. Hooker. A Systematic Approach to MDD-Based Constraint Programming. In Proceedings of CP. LNCS 6308, pp. 266-280. Springer, 2010.

- T. Hadzic, E. O'Mahony, B. O'Sullivan, and M. Sellmann. Enhanced inference for the market split problem. In Proceedings, International Conference on Tools for AI (ICTAI), pages 716-723. IEEE, 2009.

\section{1}

- D. Bergman, W.-J. van Hoeve, and J. N. Hooker. Manipulating MDD Relaxations for Combinatorial Optimization. In Proceedings of CPAIOR. LNCS 6697, pp. 20-35. Springer, 2011.

\section{2}

- A. A. Cire and W.-J. van Hoeve. MDD Propagation for Disjunctive Scheduling. In Proceedings of ICAPS, pp. 11-19. AAAI Press, 2012.

- D. Bergman, A.A. Cire, W.-J. van Hoeve, and J.N. Hooker. Variable Ordering for the Application of BDDs to the Maximum Independent Set Problem. In Proceedings of CPAIOR. LNCS 7298, pp. 34-49. Springer, 2012. 


\section{References}

\section{3}

- A. A. Cire and W.-J. van Hoeve. Multivalued Decision Diagrams for Sequencing Problems. Operations Research 61(6): 1411-1428, 2013.

- D. Bergman. New Techniques for Discrete Optimization. PhD thesis, Carnegie Mellon University, 2013.

- J. N. Hooker. Decision Diagrams and Dynamic Programming. In Proceedings of CPAIOR. LNCS 7874, pp. 94-110. Springer, 2013.

- B. Kell and W.-J. van Hoeve. An MDD Approach to Multidimensional Bin Packing. In Proceedings of CPAIOR, LNCS 7874, pp. 128-143. Springer, 2013.

\section{4}

- D. R. Morrison, E. C. Sewell, S. H. Jacobson, Characteristics of the maximal independent set ZDD, Journal of Combinatorial Optimization 28 (1) 121-139, 2014

- D. R. Morrison, E. C. Sewell, S. H. Jacobson, Solving the Pricing Problem in a Generic Branch-and-Price Algorithm using Zero-Suppressed Binary Decision Diagrams,

- D. Bergman, A. A. Cire, W.-J. van Hoeve, and J. N. Hooker. Optimization Bounds from Binary Decision Diagrams. INFORMS Journal on Computing 26(2): 253-258, 2014.

- A. A. Cire. Decision Diagrams for Optimization. PhD thesis, Carnegie Mellon University, 2014.

- D. Bergman, A. A. Cire, and W.-J. van Hoeve. MDD Propagation for Sequence Constraints. $J A I R$, Volume 50, pages 697-722, 2014.

- D. Bergman, A. A. Cire, W.-J. van Hoeve, and T. Yunes. BDD-Based Heuristics for Binary Optimization. Journal of Heuristics 20(2): 211-234, 2014. 


\section{References}

\section{4}

- D. Bergman, A. A. Cire, A. Sabharwal, H. Samulowitz, V. Saraswat, and W.-J. van Hoeve. Parallel Combinatorial Optimization with Decision Diagrams. In Proceedings of CPAIOR, LNCS 8451, pp. 351-367. Springer, 2014.

- A. A. Cire and J. N. Hooker. The Separation Problem for Binary Decision Diagrams. In Proceedings of the International Symposium on Artificial Intelligence and Mathematics (ISAIM), 2014. ]

\section{5}

- D. Bergman, A. A. Cire, and W.-J. van Hoeve. Lagrangian Bounds from Decision Diagrams. Constraints 20(3): 346-361, 2015.

- B. Kell, A. Sabharwal, and W.-J. van Hoeve. BDD-Guided Clause Generation. In Proceedings of CPAIOR, 2015.

\section{6}

- D. Bergman, A. A. Cire, W.-J. van Hoeve, and J. N. Hooker, Decision Diagrams for Optimization, Springer, to appear.

- D. Bergman, A. A. Cire, W.-J. van Hoeve, and J. N. Hooker. Discrete Optimization with Decision Diagrams. INFORMS Journal on Computing 28: 47-66, 2016. 